\title{
FAULT ANALYSIS CONSIDERING FAULT CURRENT CONTRIBUTIONS FROM DISTRIBUTED ENERGY RESOURCES
}

\author{
by \\ Adnan Arapovic, P.Eng., PMP \\ Bachelor of Applied Science, Honours Electrical Engineering, University of Waterloo, 2014 \\ An MRP presented to Ryerson University \\ in partial fulfillment of the \\ requirements for the degree of \\ Master of Engineering \\ in the program of \\ Electrical and Computer Engineering \\ Toronto, Ontario, Canada, 2018 \\ (C) Adnan Arapovic, 2018
}




\section{Author's Declaration}

I hereby declare that I am the sole author of this MRP. This is a true copy of the MRP, including any required final revisions.

I authorize Ryerson University to lend this MRP to other institutions or individuals for the purpose of scholarly research.

I further authorize Ryerson University to reproduce this MRP by photocopying or by other means, in total or in part, at the request of other institutions or individuals for the purpose of scholarly research.

I understand that my MRP may be made electronically available to the public. 
Fault Analysis Considering Fault Current Contributions from Distributed Energy Resources

Master of Engineering, 2018

Adnan Arapovic, P.Eng., PMP

Electrical and Computer Engineering

Ryerson University

With emerging concerns over climate change and the need for reduced greenhouse gas emissions, together with the growing awareness of the importance of the natural environment and the depletion of the earth's non-renewable energy resources, the generation of electricity from distributed renewable energy resources such as solar photovoltaic (PV) and wind energy has begun to expand at a rapid pace. Proliferation of converter-based distributed energy resources in distribution systems has introduced new challenges in determining the maximum possible fault currents that a power system must be able to withstand without being compromised. Therefore, it is imperative to develop the mathematical and software simulation models that approximate the response of converter-based distributed energy resources during a fault on the transmission or distribution system in order to determine the fault current contributions to the electrical grid that a transmission or distribution utility needs to reflect in their connection impact assessments. 


\section{Table of Contents}

\section{Page}

Author's Declaration.............................................................

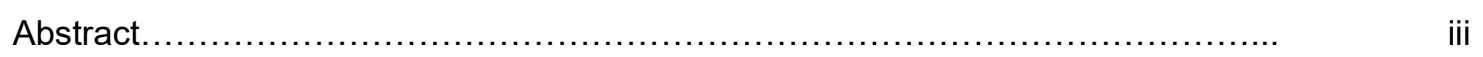

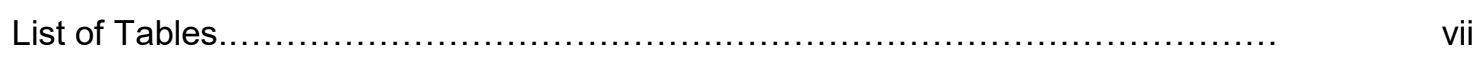

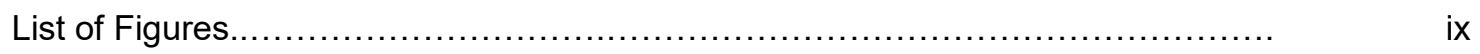

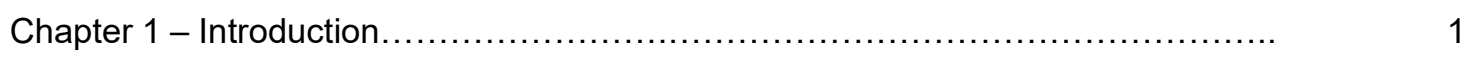

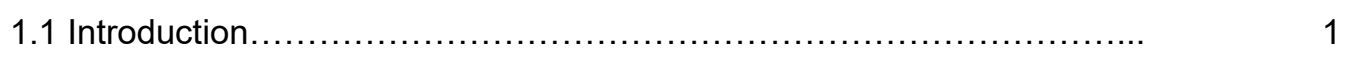

1.2 Objective of the Report........................................ 4

1.3 Outline of the Report......................................... 5

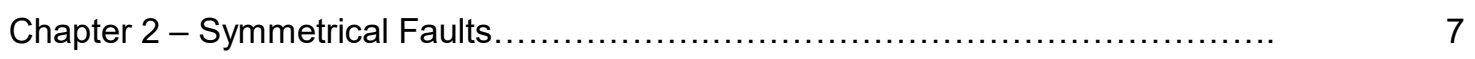

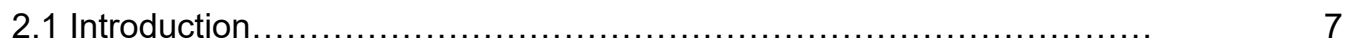

2.2 Fault Current Transients in Machines.............................. 9

2.3 Symmetrical Fault Analysis using the Bus Impedance Matrix........... 14

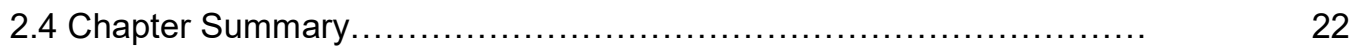

Chapter 3 - Symmetrical Components and Unsymmetrical Faults.................. 24

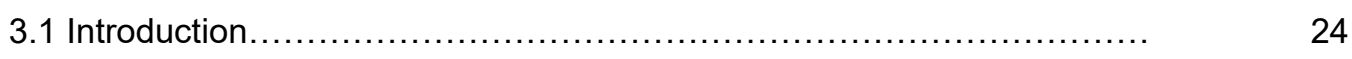

3.2 Symmetrical Components....................................... 24

3.3 Sequence Impedances and Sequence Networks..................... 30

3.4 Positive-, Negative-, and Zero-Sequence Equivalent Circuits of Generators $\ldots \ldots \ldots \ldots \ldots \ldots \ldots \ldots \ldots \ldots \ldots \ldots \ldots \ldots \ldots \ldots \ldots \ldots \ldots \ldots$

3.5 Positive-, Negative-, and Zero-Sequence Equivalent Circuits of

Transmission Lines..........................................

3.6 Positive-, Negative-, and Zero-Sequence Equivalent Circuits of

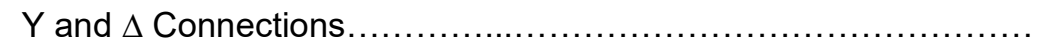

3.7 Positive-, Negative-, and Zero-Sequence Equivalent Circuits of

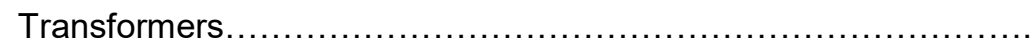


3.8 Single Line-to-Ground Fault on an Unloaded Generator................ 40

3.9 Line-to-Line Fault on an Unloaded Generator......................... 43

3.10 Double Line-to-Ground Fault on an Unloaded Generator............... 46

3.11 Unsymmetrical Faults on Power Systems......................... 49

3.12 Unsymmetrical Fault Analysis using the Bus Impedance Matrix......... 55

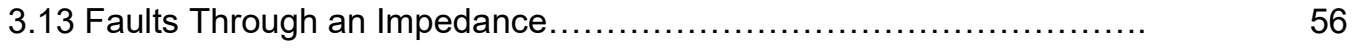

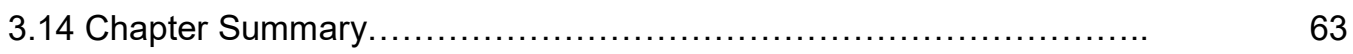

Chapter 4 - Power System Modeling and Fault Analysis of Converter-Based

Distributed Energy Resources.......................................... 65

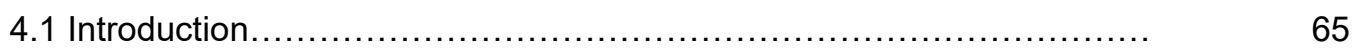

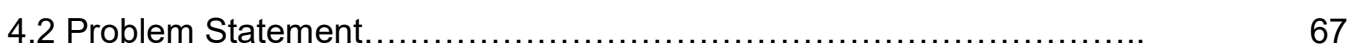

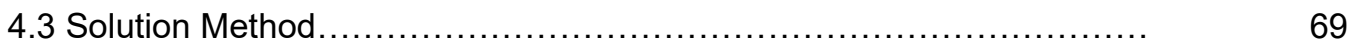

4.3.1 Fault Analysis of Converter-Based Distributed Energy

Resources for AC Side Bolted Faults................... 69

4.3.2 Fault Analysis of Converter-Based Distributed Energy

Resources for DC Side Faults.......................... 72

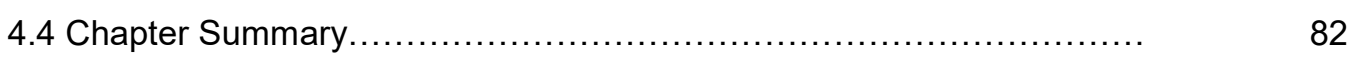

Chapter 5 - MATLAB Fault Current Analysis Program ........................... 84

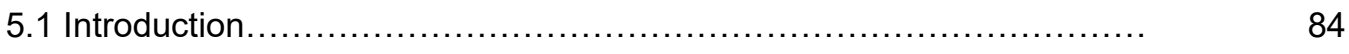

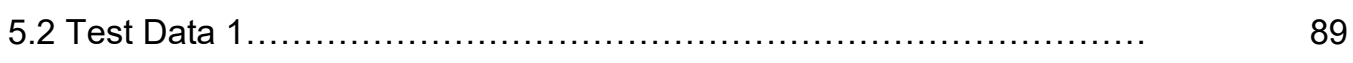

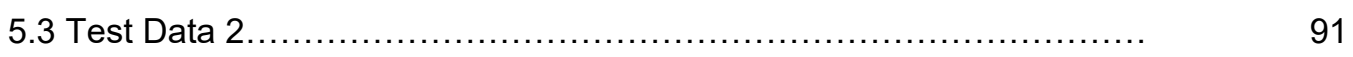

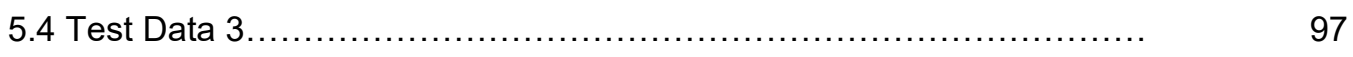

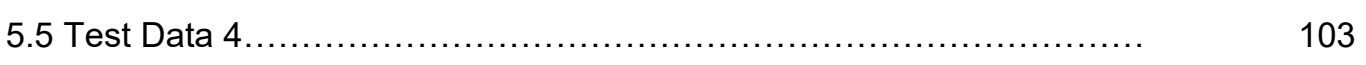

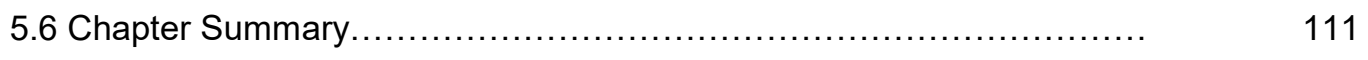

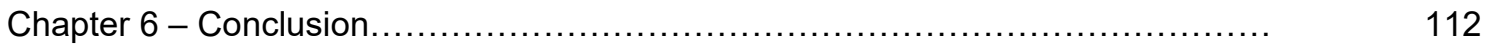

6.1 General.................................................. 112

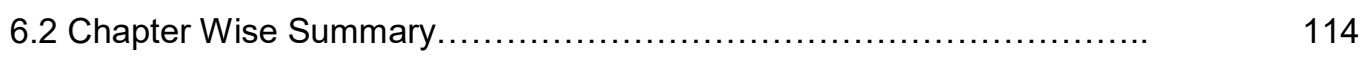

6.3 Contribution and Conclusions ...................................... 116 


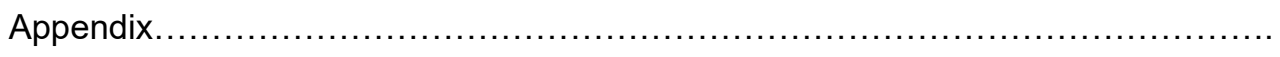

References. 


\section{List of Tables}

Table

2.1. Types of power system faults, in decreasing frequency of occurrence.

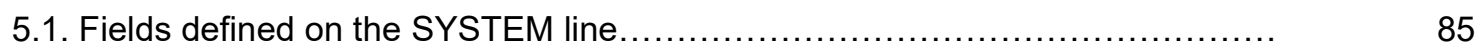

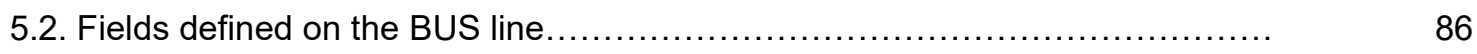

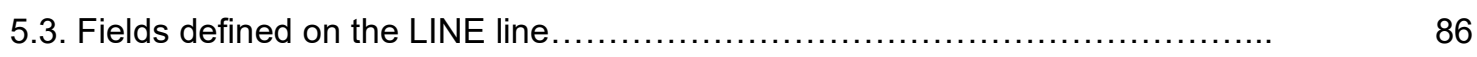

5.4. Fields defined on the GENERATOR and MOTOR lines....................... 87

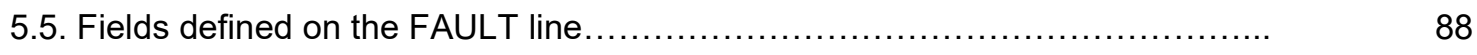

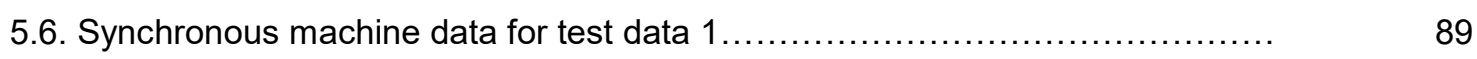

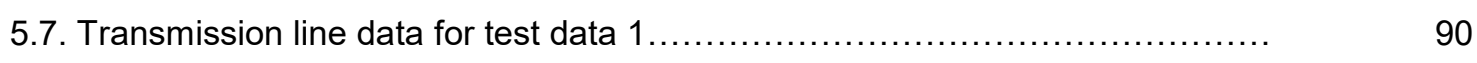

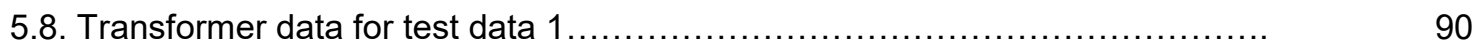

5.9. Comparison of MATLAB simulation results against published results using

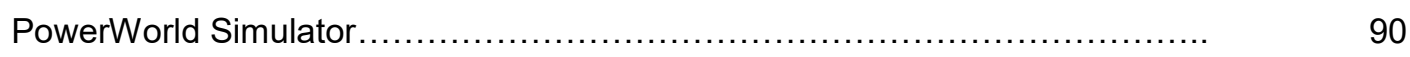

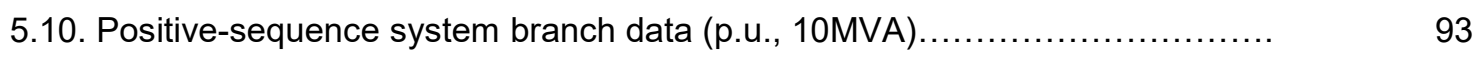

5.11. Generator impedances for momentary and interrupting duty (p.u., 10MVA)... $\quad 94$

5.12. Motor impedances for momentary and interrupting duty (p.u., 10MVA)........ 95

5.13. Comparison of MATLAB simulation results against published results from IEEE Std. 399-1997 and an ETAP short-circuit verification and validation

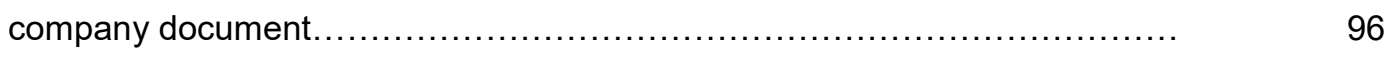

5.14. Comparison of MATLAB simulation results against calculated inverter fault

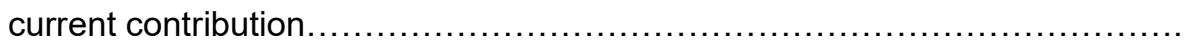

5.15. Comparison of fault current contributions from a 12.5 MVA synchronous generator and a 12.5 MVA series converter-connected generator with a gridside inverter.

5.16. Comparison of MATLAB simulation results against calculated inverter fault current contribution. 
5.17. Comparison of fault current contributions from a 12.5 MVA synchronous generator and a 12.5 MVA series converter-connected generator with a grid-

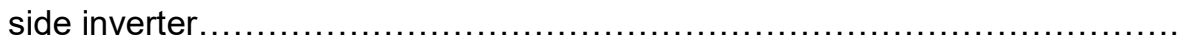




\section{List of Figures}

Figure $\quad$ Page

2.1. Current in a series $R-L$ circuit with ac voltage source $\ldots \ldots \ldots \ldots \ldots \ldots \ldots \ldots \ldots \ldots \ldots \ldots \ldots \ldots \ldots \ldots$

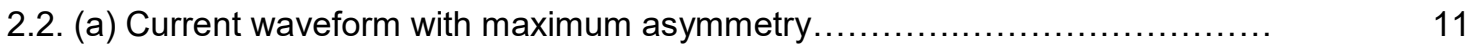

(b) Current waveform with no dc component.............................

2.3. The total fault currents as a function of time during a symmetrical three-phase fault on a synchronous generator ..................................... 12

2.4. The ac symmetrical component of the fault current............................

2.5. Single-line diagram of a four-bus three-phase power system.................. 14

2.6. The per-phase, per-unit equivalent circuit of the power system ................. 14

2.7. The equivalent circuit with a symmetrical three-phase fault on bus 2 simulated by inserting an additional voltage source $-\mathbf{V}_{f}$ in series with an existing voltage source representing the prefault voltage $\mathbf{V}_{f}$ at bus $2 \ldots \ldots \ldots \ldots \ldots \ldots \ldots \ldots \ldots$

2.8. The power system with all sources set to zero except for $-\mathbf{V}_{f}$, and with the

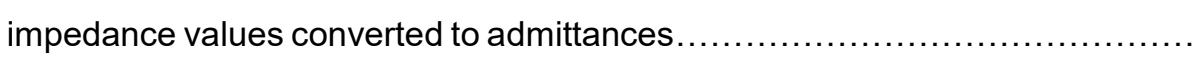

3.1. Three sets of balanced phasors which are the symmetrical components of

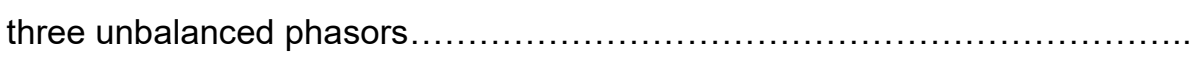

3.2. Graphical addition of the components shown in Figure 3.1 to obtain three

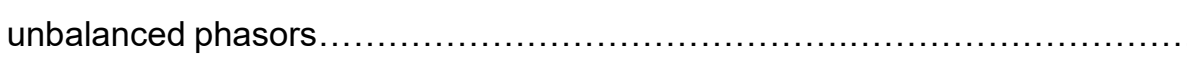

3.3. Phasor diagram illustrating the relationships among the various exponents

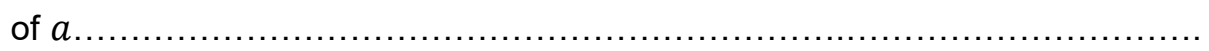

3.4. A Y-connected three-phase generator grounded through an inductive

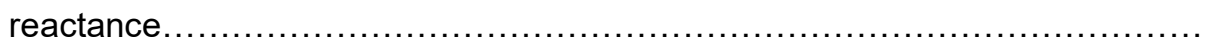

3.5. (a) A synchronous generator as seen by positive-sequence currents.

(b) The positive-sequence equivalent circuit for the generator 
(c) A synchronous generator as seen by negative-sequence currents............ 34

(d) The negative-sequence equivalent circuit for the generator.................. 34

(e) A synchronous generator as seen by zero-sequence currents................. 34

(f) The zero-sequence equivalent circuit for the generator...................... 34

3.6. (a) An ungrounded Y-connected load has no path between the neutral and the ground, thus the zero-sequence equivalent circuit has an open-circuit

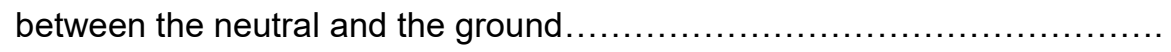

(b) A grounded Y-connected load has a short-circuit between the neutral and the ground, thus the zero-sequence equivalent circuit has a short-circuit

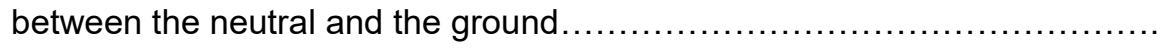

(c) A Y-connected load grounded through an impedance. The zero-sequence equivalent circuit has the impedance $3 Z_{N}$ between the neutral and the ground.

(d) A $\Delta$-connected load has no path to the ground, thus no zero-sequence currents can flow into the load. However, any zero-sequence currents inside the load can flow freely within the $\Delta$

3.7. Symbols, connection diagrams, and zero-sequence equivalent circuits for various types of three-phase transformers.

3.8. A single line-to-ground fault on phase $a$ of an unloaded generator. The generator's neutral is grounded through an inductive reactance.

3.9. For a single line-to-ground fault, the positive-, negative-, and zero-sequence networks are connected in series to determine the sequence voltages and currents in the fault.

3.10. A line-to-line fault between phases $b$ and $c$ of an unloaded generator.

3.11. For a line-to-line fault, the positive- and negative-sequence networks are connected in parallel to determine the sequence voltages and currents in the fault.

3.12. A double line-to-ground fault between phases $b, c$, and the ground of an 
unloaded generator.

3.13. For a double line-to-ground fault, the positive-, negative-, and zerosequence networks are connected in parallel to determine the sequence voltages and currents in the fault.

3.14. A simple power system with a fault at the point indicated by the $\times$

3.15. The positive-, negative-, and zero-sequence networks for the power system shown in Figure 3.14, together with their Thevenin equivalent circuits.

3.16. Connection diagram for a single line-to-ground fault on a power system.

3.17. For a single line-to-ground fault on a power system, the positive-, negative-, and zero-sequence networks are connected in series at the point of the fault $P$ to determine the sequence voltages and currents in the fault.

3.18. Connection diagram for a line-to-line fault on a power system.

3.19. For a line-to-line fault on a power system, the positive- and negativesequence networks are connected in parallel at the point of the fault $P$ to determine the sequence voltages and currents in the fault.

3.20. Connection diagram for a double line-to-ground fault on a power system.......

3.21. For a double line-to-ground fault on a power system, the positive-, negative-, and zero-sequence networks are connected in parallel at the point of the fault $P$ to determine the sequence voltages and currents in the fault.

3.22. Connection diagrams for various faults through an impedance on a power system:

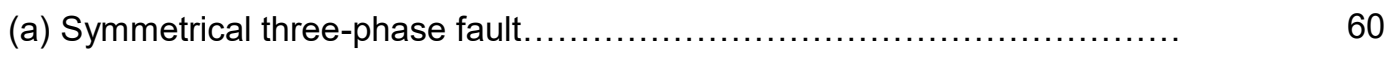

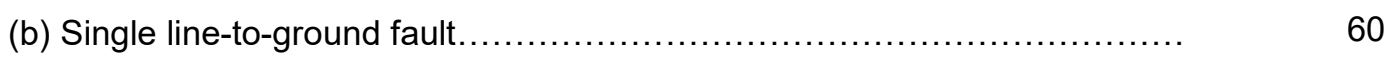

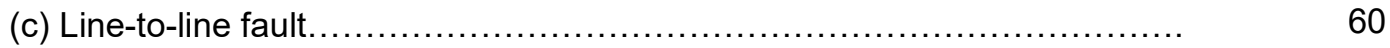

(d) Double line-to-ground fault....................................... 60

3.23. Connections of sequence networks to simulate faults through an impedance on a power system:

(a) Symmetrical three-phase fault. 
(b) Single line-to-ground fault....................................... 62

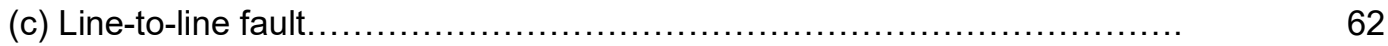

(d) Double line-to-ground fault................................................ 62

4.1. (a) Series converter-connected wind turbine generator together with flywheel

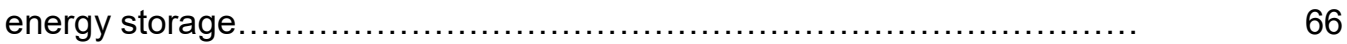

(b) Microgrid topology with series converter-connected solar PV and wind power generation options together with battery bank and supercapacitor storage options.....

4.2. Modelling the fault current contribution of a series converter-connected wind turbine generator:

(a) Initial prefault condition.............................................. 68

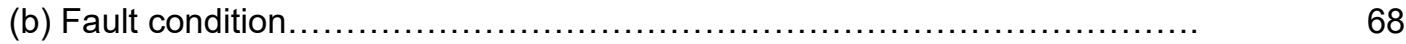

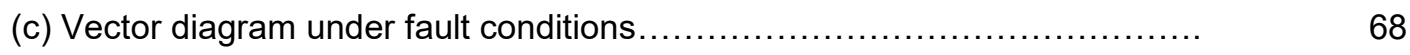

4.3. Equivalent circuit of battery for calculating fault current......................

4.4. Physical arrangement of 60-cell lead-acid storage battery mounted in three rows.......

4.5. Bus-bar reactance in ohms per foot for each conductor at 60 cycles.

4.6. Transmission line resistance and reactance per single conductor in ohms per 1000 feet at 60 cycles..........

4.7. Equivalent circuit for example calculation

4.8. Fault current-time profile of battery for example calculation.

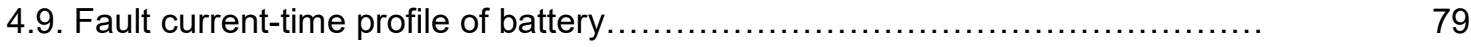

4.10. Standard approximation of fault function................................

4.11. Time to peak $t_{\mathrm{pB}}$ and rise time constant $\tau_{1 \mathrm{~B}}$ for fault of a battery $\ldots \ldots \ldots \ldots \ldots \ldots \ldots$

5.1. Sample input file to the MATLAB fault current analysis program.................

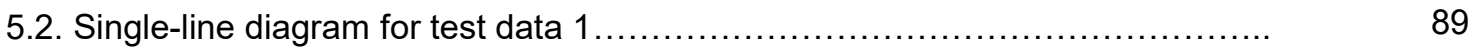

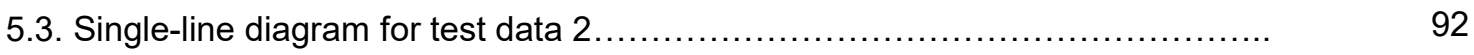

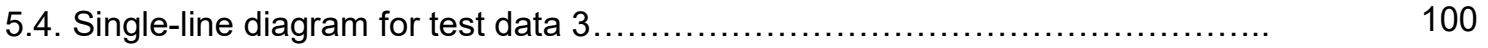




\section{Chapter 1 - Introduction}

\subsection{Introduction}

Electrical power systems are, in general, complex systems composed of a wide range of equipment devoted to generating, transmitting, and distributing electrical power to various consumption centers. Although these systems are designed to serve loads in a safe and reliable manner, the very complexity of these systems suggests that failures are unavoidable regardless of how carefully these systems have been designed. The feasibility of designing and operating a power system with zero failure rate is, if not realistic, economically unjustifiable. A fault in a circuit is any failure that interferes with the normal flow of current to the loads [1]. In most faults, a current path develops between two or more phases, or between one or more phases and the neutral (ground). Once this current path is established, it provides a short-circuit with relatively low impedance resulting in excessive current flows. If uncontrolled, these excessive current flows can cause service outage with accompanying downtime of equipment and associated inconvenience, interruption of essential facilities or vital services, extensive equipment damage, personnel injury or fatality, and possible fire damage [2].

The most common source of faults on high-voltage transmission lines is lightning [3]. When lighting strikes a phase of the transmission line, it produces a very high transient voltage that can far exceed the rated voltage of the transmission line. Although high-voltage transmission lines have strings of insulators between each phase of the transmission line and the supporting towers that carry the transmission line, when the voltage difference between the phase of the transmission line and the grounded structure of the transmission tower is large enough to ionize the air around the insulators, it creates a current path from the phase of the transmission line to the grounded structure of the transmission tower, producing an arc. The resultant condition is known as flashover. Such faults involving a single phase and ground are called single line-to-ground faults. Once the current starts flowing though the arc, it continues to do so even after the lightning disappears. However, in the case of faults involving ionized current paths known as transient faults, the transient fault will usually clear if power is removed from the transmission line for a short time and then restored.

Faults can also occur if one phase of a transmission line breaks and comes into direct contact with the ground, or if a string of insulators supporting the line breaks, allowing the line to sag to the ground. This type of fault is known as a permanent fault because, in contrast to a transient fault, it will not clear even if power is removed from the transmission line and then restored. In most power systems, approximately three-quarters of all faults are transient or permanent single line-to-ground faults [3]. There are also several other types of faults. Symmetrical three-phase faults occur when all three phases of a transmission line are shorted together. This type of fault is often the most severe and it is customary to perform only symmetrical 
three-phase fault simulations when determining the maximum possible fault currents. Line-to-line faults occur when two phases of a transmission line come into direct contact or when flashover occurs between two phases of a transmission line. Finally, double line-to-ground faults occur when two phases of a transmission line come into direct contact with each other and with the ground.

During a fault, the high current flows in the transmission line are detected by protective relays and circuit breakers on the affected transmission line are automatically opened for a brief period (approximately $1 / 3$ second or 20 cycles) to isolate the faulted portion of the transmission system from the rest of the power system and interrupt the flow of fault current in the ionized path, allowing deionization to take place and the arc conducting the current to be extinguished. The circuit breakers are then reclosed. If the power system fault was a transient fault caused by flashover, normal operation should be restored because the arc conducting the current has been extinguished. If the fault is still present on the transmission line after the circuit breakers reclose, they will open again, isolating the faulted portion of the transmission system from the rest of the system until the permanent fault can be located and cleared by a repair crew.

Proper selection of circuit breaker sizes requires knowledge of the maximum available fault current, and the circuit breakers used to provide overcurrent protection to power systems must be designed to operate properly and without damage even when the maximum possible fault currents are flowing in the power system. In addition, protective relays must be adjusted to open circuit breakers when faults occur but not under normal full-load operating conditions. The calculation of current flows under fault conditions is called fault analysis.

Since the occurrence of faults cannot always be prevented, a significant part of power system protection is devoted to detecting fault conditions in a reliable manner. To that extent, considerable capital investment is spent on interrupting equipment at all voltage levels that can withstand the maximum possible fault currents imposed on them without being destroyed and effectively isolate the smallest possible portion of the power system impacted by the fault in order to retain service to the rest of the system and reduce the amount of downtime required for equipment not belonging to the faulted portion of the power system. The selection of a circuit breaker for a power system depends not only on the current the circuit breaker must carry under normal operating conditions, but also on the maximum current it may have to carry momentarily immediately after a fault occurs (i.e., "withstand" capability) and the current it may have to interrupt at the voltage of the line in which it is placed (i.e., "interrupting" capability). Therefore, it follows that fault studies are as necessary for any power system as other fundamental system studies such as power flow studies, transient stability studies, harmonic analysis studies, etc. [4]. Fault studies can be performed at the planning stage to determine the maximum possible fault currents that equipment (cables, transformers, conductors, etc.) must be able to withstand without being destroyed. The data obtained from fault studies also serve to determine the settings of relays which control the operation of circuit breakers. For existing systems, fault 
studies are necessary in the cases of added generation, installation of extra rotating loads, system layout modifications, verification of the adequacy of existing circuit breakers, etc. "Post-mortem" analysis may also involve fault studies in order to duplicate the reasons and system conditions that lead to the system's failure.

Electric power systems have used and will continue to use synchronous machines in the foreseeable future for the generation of electricity. However, with emerging concerns over climate change and the need for reduced greenhouse gas emissions, together with the growing awareness of the importance of the natural environment and the depletion of the earth's non-renewable energy resources, the generation of electricity from distributed renewable energy resources such as solar photovoltaic (PV) and wind energy has begun to expand at a rapid pace. Generally, such generation systems either generate dc or use asynchronous machines that generate incompatible frequencies, and therefore must be interfaced to the three-phase power system through a power electronics converter. The combination of synchronous and converter isolated electrical generation systems is expected to change the behaviour of three-phase power systems following disturbances such as the occurrence of a fault on the power system. Proliferation of converterbased distributed energy resources in distribution systems has introduced new challenges in determining the maximum possible fault currents that a power system must be able to withstand without being compromised. As part of a connection impact assessment for a new generation facility, utilities require detailed information on fault characteristics of the generation sources. However, there is limited knowledge or contradictory conclusions regarding the behaviour of converter-based distributed energy resources (e.g., solar PV and wind power generation stations) during faults on transmission or distribution systems. Without accurate data and details of the fault current contributions from series converter-connected solar PV and wind generators, it is not possible to assess the risk of connecting them and hence determining the strategy and settings of the overcurrent protective devices that protect the power system. Therefore, it is imperative to develop the mathematical and software simulation models that approximate the response of converterbased distributed energy resources during a fault on the transmission or distribution system in order to determine the fault current contributions to the electrical grid that a transmission or distribution utility needs to reflect in their connection impact assessments. Theoretical models for converter-based distributed energy resources and their characteristics under fault conditions have been presented in various literary works and publications on the topic [5] - [12]. 


\subsection{Objective of the Report}

The objective of this report is to implement the theoretical models that approximate the response of converter-based distributed energy resources during a fault on the transmission or distribution system using a program developed in MATLAB for performing fault current analysis using conventional fault analysis techniques, i.e., calculating the voltages and fault currents at each bus and in each transmission line in the power system for symmetrical three-phase, single line-to-ground, line-to-line, or double line-to-ground faults during the subtransient, transient, or steady-state periods.

The MATLAB fault current analysis program is used to perform fault analysis of a power system with converter-based distributed energy resources in order to demonstrate how to implement these models to approximate the fault response of converter-based distributed energy resources within the framework of conventional fault analysis techniques and accurately simulate the fault current contributions to the electrical grid that a transmission or distribution utility needs to reflect in their connection impact assessments essential for the design and application of distribution and protective apparatuses used in these systems. 


\subsection{Outline of the Report}

The succeeding chapters and appendix comprising the remainder of the report contain the following:

The second chapter of the report, entitled "Symmetrical Faults", begins with an introduction to fault analysis, followed by a discussion of three-phase symmetrical faults and concludes with the formulation of a general procedure for calculating the voltages and fault currents at each bus and in each transmission line in a power system for symmetrical three-phase faults during the subtransient, transient, or steady-state periods. This general procedure is then illustrated by means of an example. This chapter provides the necessary background for subsequent chapters.

The third chapter of the report, entitled "Symmetrical Components and Unsymmetrical Faults", beings with an introduction to the analysis of unbalanced power systems, followed by a discussion of symmetrical components, sequence impedances and sequence networks, positive-, negative-, and zero-sequence equivalent circuits of generators, transmission lines, transformers, etc. and concludes with a discussion of single line-to-ground, line-to-line, and double line-to-ground faults on an unloaded generator which is then extended to unsymmetrical faults on more complex power systems. The relationships developed for each type of unsymmetrical fault are used to formulate a general procedure for calculating the voltages and fault currents in single line-to-ground, line-to-line, and double line-to-ground faults on a power system during the subtransient, transient, or steady-state periods. These relationships are then extended to the case where a fault occurs thorough an impedance. This chapter provides the necessary background for subsequent chapters.

The fourth chapter of the report, entitled "Power System Modelling and Fault Analysis of Converter-Based Distributed Energy Resources" begins with an introduction to the challenges imposed by the proliferation of converter-based distributed energy resources and identifies the importance of developing mathematical and software simulation models that approximate the response of converter-based distributed energy resources during a fault on the transmission or distribution system. This is subsequently followed by both a qualitative and quantitative analysis of the fault current contributions from converter-based distributed energy resources in the event of a fault on the host system (information that a transmission or distribution utility needs to reflect in their connection impact assessments). The chapter concludes with the modelling and fault analysis of converter-based distributed energy resources in the event of a fault on the dc side of a grid-side inverter.

The fifth chapter of the report, entitled "MATLAB Fault Current Analysis Program", begins with an introduction to a MATLAB program developed for calculating the voltages and fault currents at each bus and in each transmission line in a power system for symmetrical three-phase, single line-to-ground, line-to- 
line, or double line-to-ground faults during the subtransient, transient, or steady-state periods. Test data is used to verify and validate the MATLAB program against published cases and other fault current analysis software programs to ensure its technical accuracy. After comparing the results, the MATLAB program is then used to perform fault analysis of a power system with converter-based distributed energy resources in order to simulate the fault current contributions to the electrical grid that a transmission or distribution utility needs to reflect in their connection impact assessments. The input files to the MATLAB program and the corresponding MATLAB Command Window outputs for each set of test data are provided in the appendix of this report.

Finally, the sixth chapter of the report is entitled "Conclusion", followed by an appendix which contains the entire code for the MATLAB program developed for calculating the voltages and fault currents at each bus and in each transmission line in a power system for symmetrical three-phase, single line-to-ground, line-toline, or double line-to-ground faults during the subtransient, transient, or steady-state periods. The input files to the MATLAB program and the corresponding MATLAB Command Window outputs for each set of test data (from Chapter 5) are also provided in the appendix of this report. 


\section{Chapter 2 - Symmetrical Faults}

\subsection{Introduction}

A fault in a circuit is any failure that interferes with the normal flow of current to the loads [1]. In most faults, a current path develops between two or more phases, or between one or more phases and the neutral (ground). Once this current path is established, it provides a short-circuit with relatively low impedance allowing very high currents to flow through this current path, into the ground, and back into the power system though the grounded wye connections of generators in the system. The most common source of faults on high-voltage transmission lines is lightning [3]. When lighting strikes a phase of the transmission line, it produces a very high transient voltage that can far exceed the rated voltage of the transmission line. Although high-voltage transmission lines have strings of insulators between each phase of the transmission line and the supporting towers that carry the transmission line, when the voltage difference between the phase of the transmission line and the grounded structure of the transmission tower is large enough to ionize the air around the insulators, it creates a current path from the phase of the transmission line to the grounded structure of the transmission tower, producing an arc. The resultant condition is known as

flashover. Such faults involving a single phase and ground are called single line-to-ground faults. Once the current starts flowing though the arc, it continues to do so even after the lightning disappears. However, in the case of faults involving ionized current paths known as transient faults, the transient fault will usually clear if power is removed from the transmission line for a short time and then restored.

Faults can also occur if one phase of a transmission line breaks and comes into direct contact with the ground, or if a string of insulators supporting the line breaks, allowing the line to sag to the ground. This type of fault is known as a permanent fault because, in contrast to a transient fault, it will not clear even if power is removed from the transmission line and then restored. In most power systems, approximately three-quarters of all faults are transient or permanent single line-to-ground faults [3]. There are also several other types of faults. Symmetrical three-phase faults occur when all three phases of a transmission line are shorted together. Line-to-line faults occur when two phases of a transmission line come into direct contact or when flashover occurs between two phases of a transmission line. Finally, double line-to-ground faults occur when two phases of a transmission line come into direct contact with each other and with the ground. The various types of faults are listed on the next page in Table 2.1, in order of decreasing frequency of occurrence.

During a fault, the high current flows in the transmission line are detected by protective circuitry and circuit breakers on the affected transmission line are automatically opened for a brief period (approximately $1 / 3$ second or 20 cycles) to isolate the faulted portion of the transmission line from the rest of the power system and interrupt the flow of current in the ionized path, allowing deionization to take place and the arc 
conducting the current to be extinguished. The circuit breakers are then reclosed. If the power system fault was a transient fault caused by flashover, normal operation should be restored because the arc conducting the current has been extinguished. If the fault is still present on the transmission line after the circuit breakers reclose, they will open again, isolating the faulted portion of transmission line from the rest of the power system until the permanent fault can be located and cleared by a repair crew. Proper selection of circuit breaker sizes requires knowledge of the maximum available fault current, and the circuit breakers used to provide overcurrent protection to power systems must be designed to operate properly and without damage even when the maximum possible fault currents are flowing in the power system. In addition, the protective circuitry must be adjusted to open circuit breakers when faults occur but not under normal fullload operating conditions. The calculation of current flows under fault conditions is called fault analysis.

\begin{tabular}{|l|l|l|}
\hline Type of fault & Abbreviation & Category \\
\hline Single line-to-ground & SLG & Unsymmetrical \\
\hline Line-to-line & LL & Unsymmetrical \\
\hline Double line-to-ground & LLG & Unsymmetrical \\
\hline Symmetrical three-phase & 3P & Symmetrical \\
\hline
\end{tabular}

Table 2.1: Types of power system faults, in decreasing frequency of occurrence [3]. 


\subsection{Fault Current Transients in Machines}

As mentioned earlier, circuit breakers are designed to open when a fault is detected, clearing the fault and allowing the remaining part of the power system to continue operating. The selection of a circuit breaker for a power system depends not only on the current the circuit breaker must carry under normal operating conditions, but also on the maximum current it may have to carry momentarily (i.e., "withstand" capability) and the current it may have to interrupt at the voltage of the line in which it is placed (i.e., "interrupting" capability). To approach the problem of calculating the initial current when a fault occurs on a power system and develop an insight into the nature of fault currents, consider the series R-L circuit shown below in Figure 2.1. The closing of switch SW at $t=0$ seconds represents a symmetrical three-phase fault occurring at the terminals of an unloaded synchronous generator. For the time being, assume zero fault impedance, i.e., a solid or "bolted" fault. Such faults produce the maximum possible fault currents and form the basis of calculations for withstand capabilities on switching devices. The current is assumed to be zero before the switch is closed, and the phase shift $\alpha$ determines the magnitude of the applied voltage $V_{\max } \cos (\omega t+\alpha)$ when the circuit is closed at $t=0$ seconds.

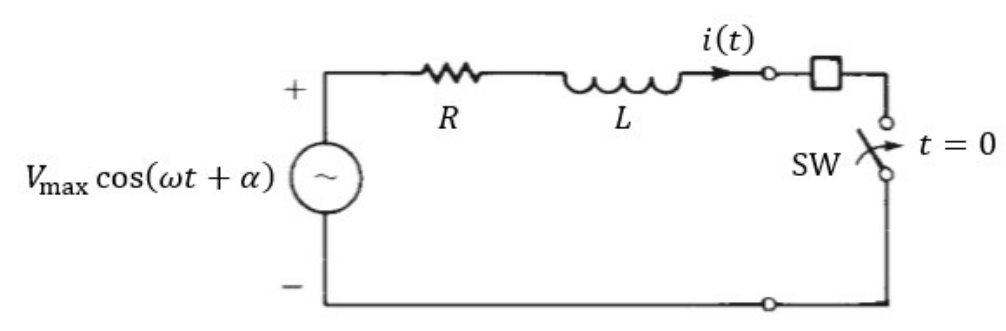

Figure 2.1: Current in a series R-L circuit with ac voltage source [13].

If the instantaneous voltage is zero volts and increasing in a positive direction when the switch is closed, then $\alpha=-\pi / 2 \mathrm{rad}$. If the instantaneous voltage is at its positive maximum value when the switch is closed, then $\alpha=0$ rad. The differential equation is

$$
V_{\max } \cos (\omega t+\alpha)=R i+L \frac{d i}{d t}
$$

The solution of this equation is

$$
i(t)=\frac{V_{\max }}{|Z|}\left[\cos (\omega t+\alpha-\theta)-e^{-R t / L} \cos (\alpha-\theta)\right] \mathrm{A}
$$


where $|Z|=\sqrt{R^{2}+(\omega L)^{2}} \Omega$ and $\theta=\tan ^{-1}(\omega L / R)$ rad. In transmission systems, $\omega L \gg R$. For example, a 100 MVA, 0.85 power factor synchronous generator may have a ratio of reactance $(X)$ to resistance $(R)$, i.e., $X / R$ ratio, of 110 and is therefore typically modeled by an internal generated voltage in series with an inductance, while a transformer of the same rating may have an $X / R$ ratio of 45 [14], [15]. The $X / R$ ratios in low-voltage systems are of the order of 2-8 [14], [15]. For present discussions, assume a high $X / R$ ratio, i.e., $\theta \approx \pi / 2 \mathrm{rad}$.

The first term of Equation (2.2) varies sinusoidally with time and is called the ac symmetrical component or steady-state component of the current. The second term is nonperiodic and decays exponentially with a time constant $L / R$. This nonperiodic term is called the dc component of the current. If the value of the steady-state component is not zero when $t=0$ seconds, the dc component appears in the solution to satisfy the physical condition of zero current at the instant of closing the switch. In other words, the dc component is created at the moment of the fault in order for the current to be continuous at that time since a current cannot change instantaneously in an inductive circuit. Note that this term does not exist if the switch is closed at a point on the voltage wave such that $\alpha-\theta= \pm \pi / 2 \mathrm{rad}$, i.e., $\alpha=0$ or $\pi$ rad. However, if the switch is closed at a point on the voltage wave such that $\alpha-\theta=0 \mathrm{rad}$, i.e., $\alpha=\pi / 2 \mathrm{rad}$, or $\alpha-$ $\theta=\pi \mathrm{rad}$, i.e., $\alpha=-\pi / 2 \mathrm{rad}$, the dc component has its maximum initial value, which is equal to the maximum value of the steady-state component (sometimes called the doubling effect [14], [15]). These two situations are shown on the next page in Figure 2.2(a) and (b). The dc component may have any value from $0 \mathrm{~A}$ to $V_{\max } /|Z|$, depending on the instantaneous value of the ac voltage source when the circuit is closed and on the power factor of the circuit. At the instant of closing the switch, the dc and steady-state components always have the same magnitude but are opposite in sign to express the zero value of current then existing. Since the instantaneous values of current at the moment of the fault are different in each phase, the magnitude of the dc component will be different in each phase as shown on page 12 in Figure 2.3. These dc components of current decay quickly, but they initially average approximately 50 or 60 percent of the ac current flow the instant after the fault occurs. As a rule of thumb, the total asymmetrical rms current is therefore typically 1.5 or 1.6 times the size of the subtransient currents alone [3], [16]. All power equipment (transformers, circuit breakers, etc.) must be designed to withstand this extremely high current level for a brief period without damage. This is known as the "withstand" capability of an equipment or device. 

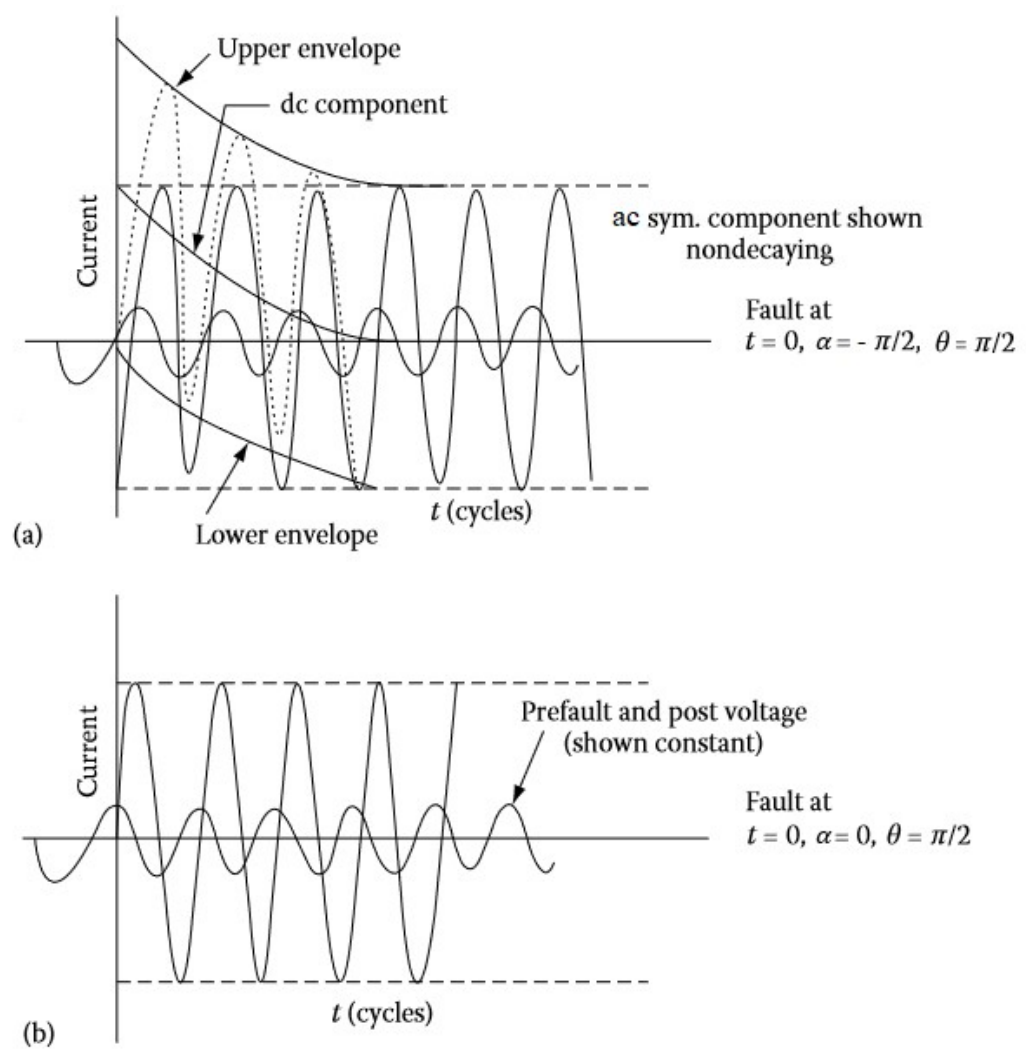

Figure 2.2: (a) Current waveform with maximum asymmetry. (b) Current waveform with no dc component [14]. 

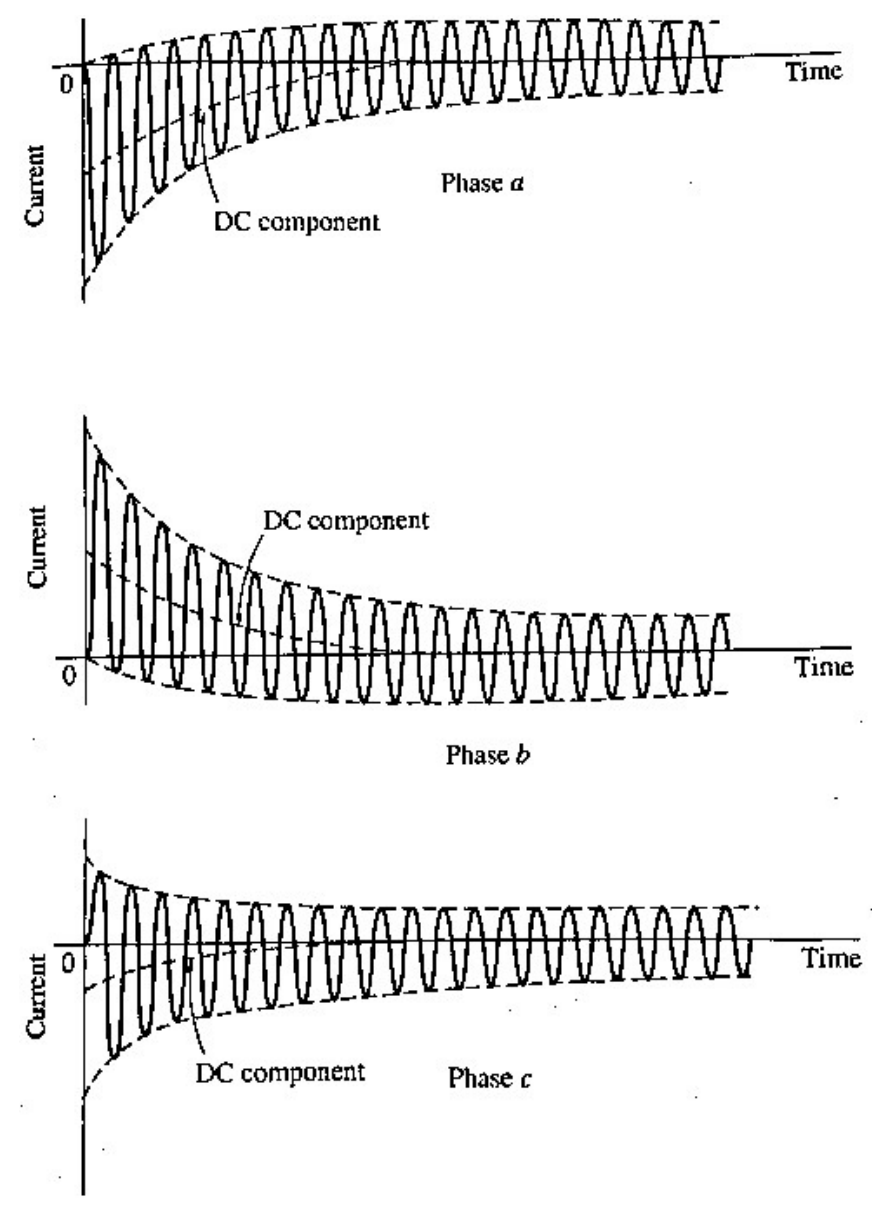

Figure 2.3: The total fault currents as a function of time during a symmetrical three-phase fault on a synchronous generator [3].

The ac symmetrical component of the fault current by itself is shown on next page in Figure 2.4. The ac symmetrical component of current starts out very high, and decays to a steady-state value in two stages. The first period of very rapid decay is called the subtransient period and it lasts only a few cycles. The ac rms current flowing during the subtransient period is called the subtransient current. The subtransient current is basically associated with the time constants of the amortisseur or damper windings of generators and motors and is often as much as 10 times the size of the steady-state rms current [3]. The second period of slower decay is called the transient period and it lasts for 0.5 to 1.0 second. The ac rms current flowing during the transient period is called the transient current. The transient current is basically associated with the time constants of the field windings of generators and motors and is often as much as 5 times the size of the steady-state rms current [3]. After the transient period, the ac symmetrical component of current reaches the steady-state period. In general, the subtransient currents are needed to specify the "withstand" capability of any circuit breakers in the system, while the transient currents are needed to specify the "interrupting" capability of the circuit breakers. The reactances of generators and motors in the subtransient 
period are called subtransient reactances $\left(X^{\prime \prime}\right)$. The reactances of generators and motors in the transient period are called transient reactances $\left(X^{\prime}\right)$. The reactances of generators and motors in the steady-state period are the machines' synchronous reactances $\left(X_{S}\right)$. The resistances and reactances of transmission lines, transformers, and other static components do not change during the fault transients.

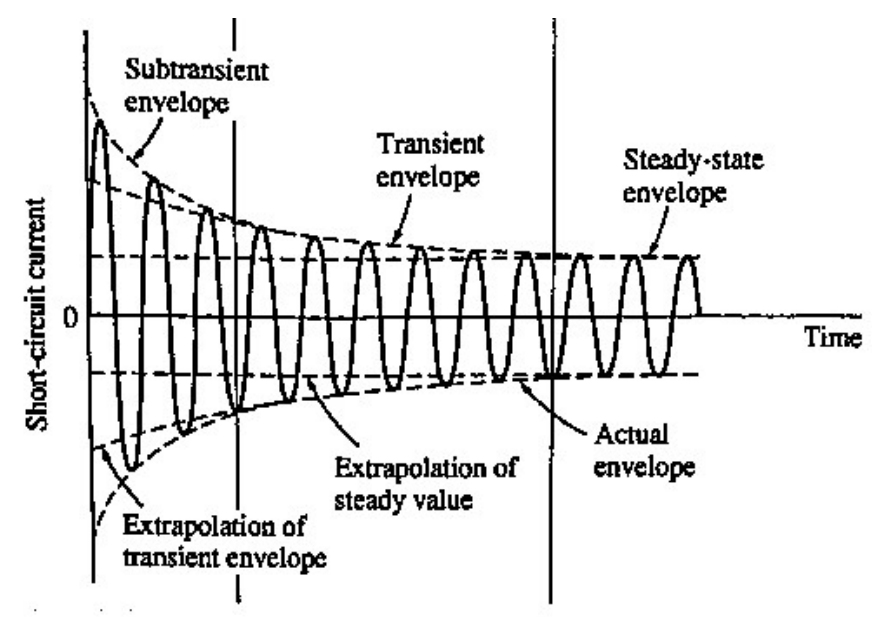

Figure 2.4: The ac symmetrical component of the fault current [3]. 


\subsection{Symmetrical Fault Analysis using the Bus Impedance Matrix}

The discussion of fault current calculations thus far has been limited to simple circuits, but it will now be extended to an $\mathrm{N}$-bus power system. To solve for the fault currents in a power system, a new voltage source will be introduced in the power system to represent the effects of a fault at a bus. By solving for the currents introduced by this additional voltage source, it will automatically be solving for the fault currents that flow at that bus. To understand this approach, consider the following example borrowed from [1] as shown below in Figure 2.5. The example proceeds to develop the general equations by first creating a per-phase, perunit equivalent circuit of the power system using the subtransient reactance $X^{\prime \prime}$ instead of the synchronous reactance $X_{S}$ for each synchronous machine as shown below in Figure 2.6. For simplicity, the power system is assumed to be initially unloaded. In the absence of loads, no prefault currents flow between buses and the voltage at every bus (and the internal voltage of each generator) will be the same as the prefault voltage at the faulted bus, denoted by the symbol $\mathbf{V}_{f}$.

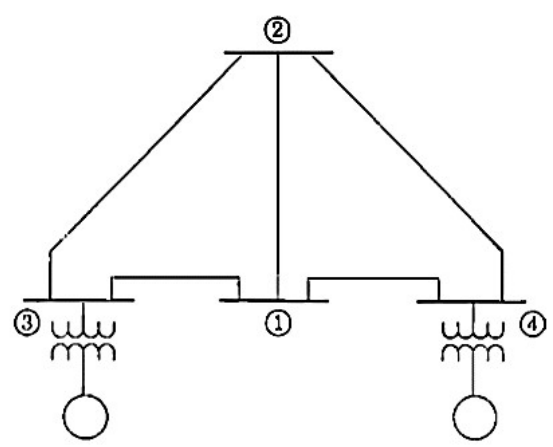

Figure 2.5: Single-line diagram of a four-bus three-phase power system [1].

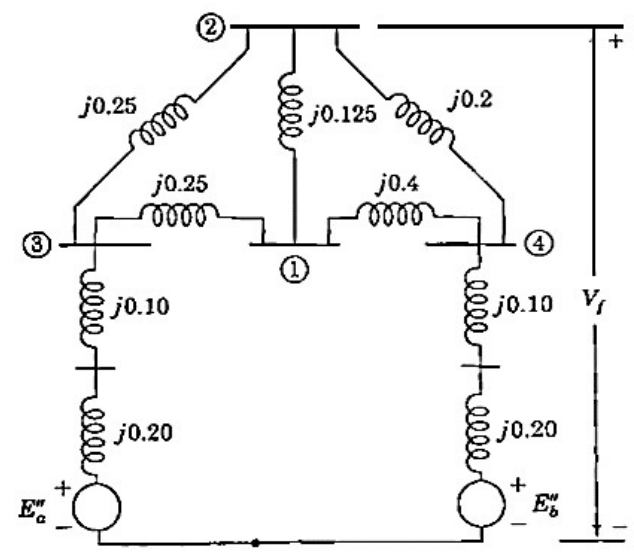

Figure 2.6: The per-phase, per-unit equivalent circuit of the power system [1]. Note that the subtransient reactances are used instead of the synchronous reactances for each synchronous machine. 
Since the prefault voltage at bus 2 is already $\mathbf{V}_{f}$, a voltage source $\mathbf{V}_{f}$ can be inserted at bus 2 without causing current to flow in the power system as shown below in Figure 2.7. If a symmetrical three-phase fault occurs on bus 2 , this is equivalent to inserting an additional voltage source $-\mathbf{V}_{f}$ in series with the existing voltage source representing the prefault voltage $\mathbf{V}_{f}$ at bus 2 , making the total voltage at bus 2 become zero and thereby simulating a symmetrical three-phase fault on bus 2 . With this additional voltage source inserted, there will now be a fault current $\mathbf{I}_{f}^{\prime \prime}$. This fault current is entirely due to the insertion of the new voltage source; therefore, superposition can be used to analyze the effects of only the new voltage source on the power system. The resulting current flow will be the current for the entire power system, because the other sources in the power system produced a net zero current.

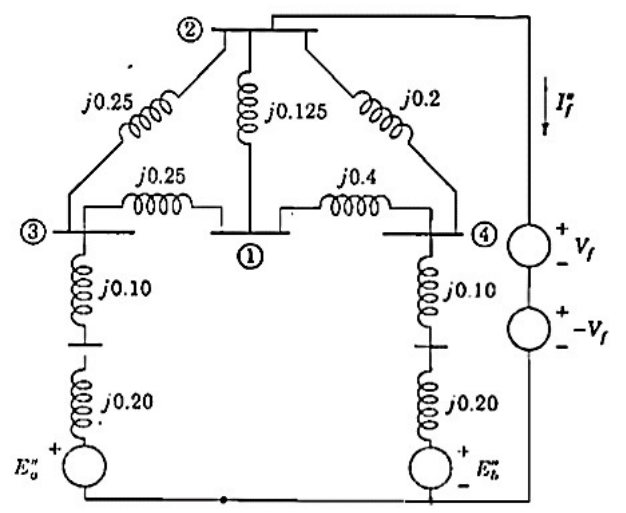

Figure 2.7: The equivalent circuit with a symmetrical three-phase fault on bus 2 simulated by inserting an additional voltage source $-\mathbf{V}_{f}$ in series with an existing voltage source representing the prefault voltage $\mathbf{V}_{f}$ at bus 2 [1]. When the additional voltage source $-\mathbf{V}_{f}$ is inserted, the voltage at bus 2 becomes zero and a fault current $\mathbf{I}_{f}^{\prime \prime}$ flows. Since there was no fault current before inserting the additional voltage source, the fault current now flowing is entire due to the insertion of the new voltage source.

If all the voltage sources except for $-\mathbf{V}_{f}$ are set to zero and the impedance values are converted to admittances, the power system appears as shown on the next page in Figure 2.8. From this figure, it is then possible to construct the bus admittance matrix $\mathbf{Y}_{\text {bus }}$. The bus admittance matrix $\mathbf{Y}_{\text {bus }}$ for an $N$-bus power system has the following form:

$$
\mathbf{Y}_{\text {bus }}=\left[\begin{array}{cccc}
Y_{11} & Y_{12} & \cdots & Y_{1 N} \\
Y_{21} & Y_{22} & \ldots & Y_{2 N} \\
\vdots & \vdots & \vdots & \vdots \\
Y_{N 1} & Y_{N 2} & \ldots & Y_{N N}
\end{array}\right]
$$


The order of the subscripts of the elements $Y_{i j}$ of $\mathbf{Y}_{\text {bus }}$ is effect-cause, i.e., the first subscript represents the bus at which the current is being expressed and the second subscript represents the voltage causing this component of current. The rules for determining the elements of $\mathbf{Y}_{\text {bus }}$ are as follows:

- The diagonal elements $Y_{j j}$ are equal to the sum of all admittances directly connected to bus $j$ and are called the self-admittances (or driving point admittances) of the buses.

- The off-diagonal elements $Y_{i j}$ are equal to the negative of the sum of all admittances directly connected between buses $i$ and $j$ and are called the mutual admittances (or transfer admittances) of the buses.

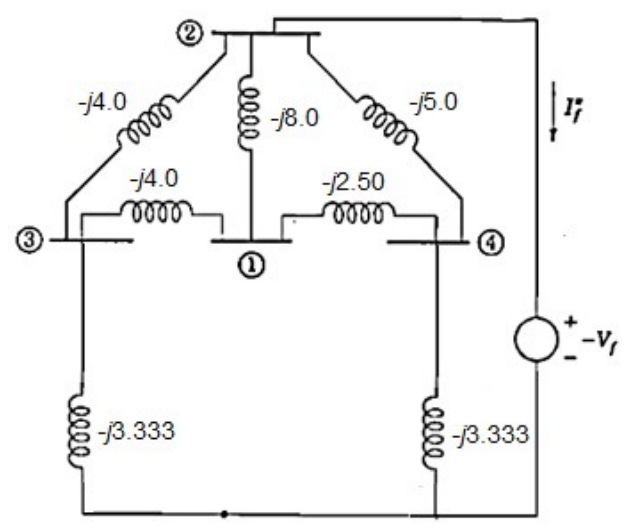

Figure 2.8: The power system with all sources set to zero except for $-\mathbf{V}_{f}$, and with the impedance values converted to admittances.

The nodal equation describing this power system is

$$
\mathbf{Y}_{\mathrm{bus}} \mathbf{V}=\mathbf{I}
$$

With all other voltage sources set to zero, the voltage at bus 2 is $-\mathbf{V}_{f}$, and the current entering bus 2 is $-\mathbf{I}_{f}^{\prime \prime}$. Therefore, the nodal equation becomes

$$
\left[\begin{array}{llll}
Y_{11} & Y_{12} & Y_{13} & Y_{14} \\
Y_{21} & Y_{22} & Y_{23} & Y_{24} \\
Y_{31} & Y_{32} & Y_{33} & Y_{34} \\
Y_{41} & Y_{42} & Y_{43} & Y_{44}
\end{array}\right]\left[\begin{array}{c}
\Delta \mathbf{V}_{1} \\
-\mathbf{V}_{f} \\
\Delta \mathbf{V}_{3} \\
\Delta \mathbf{V}_{4}
\end{array}\right]=\left[\begin{array}{c}
0 \\
-\mathbf{I}_{f}^{\prime \prime} \\
0 \\
0
\end{array}\right]
$$

where $\Delta \mathbf{V}_{1}, \Delta \mathbf{V}_{3}$, and $\Delta \mathbf{V}_{4}$ are the changes in the voltages at those buses due to the current $-\mathbf{I}_{f}^{\prime \prime}$ injected at bus 2 by the fault. The solution to Equation (2.5) is 


$$
\begin{gathered}
\mathbf{V}=\mathbf{Y}_{\text {bus }}^{-1} \mathbf{I}=\mathbf{Z}_{\text {bus }} \mathbf{I} \\
{\left[\begin{array}{c}
\Delta \mathbf{V}_{1} \\
-\mathbf{V}_{f} \\
\Delta \mathbf{V}_{3} \\
\Delta \mathbf{V}_{4}
\end{array}\right]=\left[\begin{array}{llll}
Z_{11} & Z_{12} & Z_{13} & Z_{14} \\
Z_{21} & Z_{22} & Z_{23} & Z_{24} \\
Z_{31} & Z_{32} & Z_{33} & Z_{34} \\
Z_{41} & Z_{42} & Z_{43} & Z_{44}
\end{array}\right]\left[\begin{array}{c}
0 \\
-\mathbf{I}_{f}^{\prime \prime} \\
0 \\
0
\end{array}\right]}
\end{gathered}
$$

where $\mathbf{Z}_{\text {bus }}=\mathbf{Y}_{\text {bus }}^{-1}$. The order of the subscripts of the elements $Z_{i j}$ of $\mathbf{Z}_{\text {bus }}$ is effect-cause, i.e., the first subscript represents the bus at which the voltage is being expressed and the second subscript represents the current causing this component of voltage. Since only bus 2 has current injected at it, it is evident that Equation (2.7) reduces to

$$
\begin{aligned}
& \Delta \mathbf{V}_{1}=-Z_{12} \mathbf{I}_{f}^{\prime \prime} \\
& -\mathbf{V}_{f}=-Z_{22} \mathbf{I}_{f}^{\prime \prime} \\
& \Delta \mathbf{V}_{3}=-Z_{32} \mathbf{I}_{f}^{\prime \prime} \\
& \Delta \mathbf{V}_{4}=-Z_{42} \mathbf{I}_{f}^{\prime \prime}
\end{aligned}
$$

In other words, the fault current at bus 2 is simply the prefault voltage $\mathbf{V}_{f}$ at bus 2 divided by $Z_{22}$, the selfimpedance (or driving point impedance) at bus 2 .

$$
\mathbf{I}_{f}^{\prime \prime}=\frac{\mathbf{V}_{f}}{Z_{22}}
$$

The voltage difference at each of the buses in the power system due to the fault current can be calculated by substituting Equation (2.9) into Equations (2.8):

$$
\begin{array}{lc}
\Delta \mathbf{V}_{1} & -Z_{12} \mathbf{I}_{f}^{\prime \prime} \\
\Delta \mathbf{V}_{2} & -\mathbf{V}_{f} \\
\Delta \mathbf{V}_{3}= & -Z_{32} \mathbf{I}_{f}^{\prime \prime} \\
\Delta \mathbf{V}_{4} & -Z_{42} \mathbf{I}_{f}^{\prime \prime}
\end{array}=\left[\begin{array}{c}
-\frac{Z_{12}}{Z_{22}} \mathbf{V}_{f} \\
-\mathbf{V}_{f} \\
-\frac{Z_{32}}{Z_{22}} \mathbf{V}_{f} \\
-\frac{Z_{42}}{Z_{22}} \mathbf{V}_{f}
\end{array}\right]
$$

If it is assumed that the power system was operating at no-load conditions before the fault, it is relatively straightforward to calculate the voltages at every bus during the fault. At no load, the voltage will be the same at every bus in the power system, hence the voltage at every bus in the power system is $\mathbf{V}_{f}$. The 
change in voltage at every bus caused by the fault current $-\mathbf{I}_{f}^{\prime \prime}$ is given by Equation (2.10), hence the total voltage at each bus during the fault can be calculated from

$$
\begin{gathered}
{\left[\begin{array}{l}
\mathbf{V}_{1} \\
\mathbf{V}_{2} \\
\mathbf{V}_{3} \\
\mathbf{V}_{4}
\end{array}\right]=\left[\begin{array}{l}
\mathbf{V}_{f} \\
\mathbf{V}_{f} \\
\mathbf{V}_{f} \\
\mathbf{V}_{f}
\end{array}\right]+\left[\begin{array}{l}
\Delta \mathbf{V}_{1} \\
\Delta \mathbf{V}_{2} \\
\Delta \mathbf{V}_{3} \\
\Delta \mathbf{V}_{4}
\end{array}\right]} \\
{\left[\begin{array}{l}
\mathbf{V}_{1} \\
\mathbf{V}_{2} \\
\mathbf{V}_{3} \\
\mathbf{V}_{4}
\end{array}\right]=\left[\begin{array}{l}
\mathbf{V}_{f} \\
\mathbf{V}_{f} \\
\mathbf{V}_{f} \\
\mathbf{V}_{f}
\end{array}\right]+\left[\begin{array}{c}
-\frac{Z_{12}}{Z_{22}} \mathbf{V}_{f} \\
-\mathbf{V}_{f} \\
-\frac{Z_{32}}{Z_{22}} \mathbf{V}_{f} \\
-\frac{Z_{42}}{Z_{22}} \mathbf{V}_{f}
\end{array}\right]=\left[\begin{array}{c}
1-\frac{Z_{12}}{Z_{22}} \\
0 \\
1-\frac{Z_{32}}{Z_{22}} \\
1-\frac{Z_{42}}{Z_{22}}
\end{array}\right] \mathbf{V}_{f}}
\end{gathered}
$$

Therefore, it is possible to calculate the voltage at every bus in the power system during the fault from a knowledge of the prefault voltage $\mathbf{V}_{f}$ at the faulted bus and the bus impedance matrix $\mathbf{Z}_{\text {bus }}$. Once these bus voltages are known, it is then also possible to calculate the fault current flowing in any transmission line during the fault using the bus voltages and the bus admittance matrix $\mathbf{Y}_{\text {bus }}$.

The general procedure for calculating the voltages and fault currents at each bus and in each transmission line in the power system during a symmetrical three-phase fault is as follows [3]:

1. Create a per-phase, per-unit equivalent circuit for the power system. Include subtransient reactances $X^{\prime \prime}$ of each synchronous machine if the subtransient fault currents are desired and include the transient reactances $X^{\prime}$ of each synchronous machine if the transient fault currents are desired. Also include the subtransient reactances of each induction motor if the subtransient fault currents are desired and ignore the inductions motors completely if the transient fault currents are desired. (This is because when a fault develops on a power system with a synchronous motor, the motor becomes a temporary generator by converting its energy of rotation back into electrical power, which is supplied to the power system. Since a synchronous motor is physically the same as a synchronous generator, it also has a subtransient reactance $X^{\prime \prime}$ and transient reactance $X^{\prime}$ that must be considered in analyzing fault currents flowing in the power system. In contrast, an induction motor is simply an AC machine that has only amortisseur or damper windings on its rotor. Since damper windings are the primary source of current during the subtransient period, the induction motors connected to a power system should be considered in analyzing subtransient fault currents flowing in the power system. However, since the 
currents in a damper winding are of negligible importance during the transient and steady-state periods of faults, induction motors may be ignored in analyzing fault currents after the subtransient period ends.)

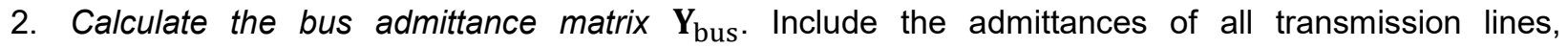
transformers, etc., between buses, including the admittances of the loads or generators themselves at each bus.

3. Calculate the bus impedance matrix $\mathbf{Z}_{\mathrm{bus}}$. The bus impedance matrix $\mathbf{Z}_{\mathrm{bus}}$ is the inverse of the bus admittance matrix $\mathbf{Y}_{\text {bus. }}$ (MATLAB can be used to perform this calculation.)

4. Assume that the power system is at no-load conditions and determine the voltage at every bus. At noload conditions, the voltage at every bus will be the same, since otherwise a current would flow between buses. This voltage will be the same as the internal voltage of the generators in the system. It is the prefault voltage in the power system, denoted by the symbol $\mathbf{V}_{f}$.

5. Calculate the current at the faulted bus. The current at the faulted bus can be calculated directly from a knowledge of $\mathbf{V}_{f}$ and $\mathbf{Z}_{\text {bus. }}$. If bus $i$ is faulted, the fault current will be

$$
\mathbf{I}_{f, i}^{\prime \prime}=\frac{\mathbf{V}_{f}}{Z_{i i}} \mathrm{pu}
$$

6. Calculate the voltages at each bus during the fault. The voltage at bus $j$ during a symmetrical threephase fault at bus $i$ is given by the equation

$$
\mathbf{V}_{j}=\left(1-\frac{Z_{j i}}{Z_{i i}}\right) \mathbf{v}_{f} \mathrm{pu}
$$

7. Calculate the currents in any desired transmission lines during the fault. The current flowing through a transmission line between bus $i$ and bus $j$ can be calculated from the equation

$$
\mathbf{I}_{i j}=-Y_{i j}\left(\mathbf{V}_{i}-\mathbf{V}_{j}\right) \mathrm{pu}
$$

The above general procedure for calculating the voltages and fault currents at each bus and in each transmission line in the power system during a symmetrical three-phase fault is illustrated by extending the earlier example borrowed from [1] as follows: 
1. Create a per-phase, per-unit equivalent circuit for the power system. This step was completed on page 6. The resulting per-phase, per-unit equivalent circuit is shown in Figure 2.6.

2. Calculate the bus admittance matrix $\mathbf{Y}_{\text {bus }}$. The rules for determining the elements of $\mathbf{Y}_{\text {bus }}$ was described earlier. The MATLAB statement required to initialize $\mathbf{Y}_{\text {bus }}$ is

$$
\begin{aligned}
& \gg \text { Ybus }=\ldots \\
& \begin{array}{ccccc}
{[-j \star 14.500} & j \star 8.000 & j * 4.000 & j \star 2.500 ; & \ldots \\
j \star 8.000 & -j \star 17.000 & j * 4.000 & j * 5.000 ; & \ldots \\
j \star 4.000 & j \star 4.000 & -j * 11.333 & 0 ; \ldots & \\
j \star 2.500 & j \star 5.000 & 0 & -j \star 10.833] ;
\end{array}
\end{aligned}
$$

3. Calculate the bus impedance matrix $\mathbf{Z}_{\text {bus. }}$. The bus impedance matrix $\mathbf{Z}_{\text {bus }}$ is the inverse of the bus admittance matrix $\mathbf{Y}_{\text {bus. }}$ (MATLAB can be used to perform this calculation.)

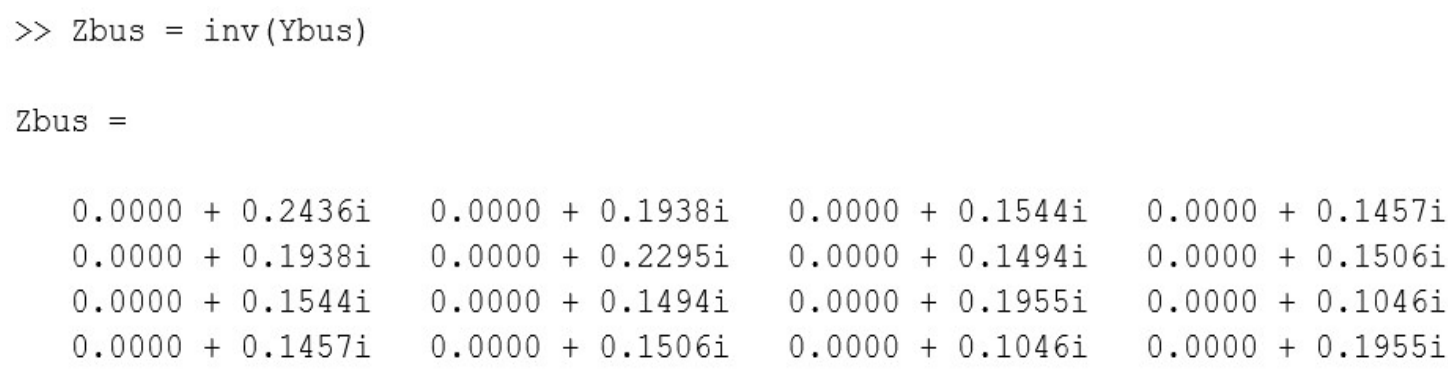

4. Assume that the power system is at no-load conditions and determine the voltage at every bus. For this power system, the no-load voltage at every bus, which will be equal to the prefault voltage at the bus, will be $\mathbf{V}_{f}=1.00 \angle 0^{\circ} \mathrm{pu}$.

5. Calculate the current at the faulted bus. The current at the faulted bus is

$$
\mathbf{I}_{f, 2}^{\prime \prime}=\frac{\mathbf{V}_{f}}{Z_{22}}=\frac{1.00 \angle 0^{\circ}}{j 0.2295}=4.3573 \angle-90^{\circ} \mathrm{pu}
$$

6. Calculate the voltages at each bus during the fault. The voltage at bus $j$ during a symmetrical threephase fault at bus $i$ is given by the equation

$$
\mathbf{v}_{j}=\left(1-\frac{Z_{j i}}{Z_{i i}}\right) \mathbf{v}_{f}
$$




$$
\begin{aligned}
& \mathbf{V}_{1}=\left(1-\frac{Z_{12}}{Z_{22}}\right) \mathbf{V}_{f}=\left(1-\frac{j 0.1938}{j 0.2295}\right)\left(1.00 \angle 0^{\circ}\right)=0.1556 \angle 0^{\circ} \mathrm{pu} \\
& \mathbf{V}_{2}=0.0 \angle 0^{\circ} \mathrm{pu} \\
& \mathbf{V}_{3}=\left(1-\frac{Z_{32}}{Z_{22}}\right) \mathbf{V}_{f}=\left(1-\frac{j 0.1494}{j 0.2295}\right)\left(1.00 \angle 0^{\circ}\right)=0.3490 \angle 0^{\circ} \mathrm{pu} \\
& \mathbf{V}_{4}=\left(1-\frac{Z_{42}}{Z_{22}}\right) \mathbf{V}_{f}=\left(1-\frac{j 0.1506}{j 0.2295}\right)\left(1.00 \angle 0^{\circ}\right)=0.3438 \angle 0^{\circ} \mathrm{pu}
\end{aligned}
$$

7. Calculate the currents in any desired transmission lines during the fault. The current flowing through transmission line $3-1$ can be calculated from

$$
\mathbf{I}_{31}=-Y_{31}\left(\mathbf{V}_{3}-\mathbf{V}_{1}\right)=(-j 4.0)\left(0.3490 \angle 0^{\circ}-0.1556 \angle 0^{\circ}\right)=0.7736 \angle-90^{\circ} \mathrm{pu}
$$

The fault current contributions to bus 2 by the adjacent unfaulted buses are

From bus 1:

$$
\mathbf{I}_{12}=-Y_{12}\left(\mathbf{V}_{1}-\mathbf{V}_{2}\right)=(-j 8.0)\left(0.1556 \angle 0^{\circ}-0.0 \angle 0^{\circ}\right)=1.2448 \angle-90^{\circ} \mathrm{pu}
$$

From bus 3:

$$
\mathbf{I}_{32}=-Y_{32}\left(\mathbf{V}_{3}-\mathbf{V}_{2}\right)=(-j 4.0)\left(0.3490 \angle 0^{\circ}-0.0 \angle 0^{\circ}\right)=1.3960 \angle-90^{\circ} \mathrm{pu}
$$

From bus 4:

$$
\mathbf{I}_{42}=-Y_{42}\left(\mathbf{V}_{4}-\mathbf{V}_{2}\right)=(-j 5.0)\left(0.3438 \angle 0^{\circ}-0.0 \angle 0^{\circ}\right)=1.7190 \angle-90^{\circ} \mathrm{pu}
$$

Except for round-off errors, the sum of these fault current contributions is equal to $\mathbf{I}_{f, 2}^{\prime \prime}$ as expected. 


\subsection{Chapter Summary}

A fault in a circuit is any failure that interferes with the normal flow of current to the loads. In most faults, a current path forms between two or more phases, or between one or more phases and the neutral (ground). This current path has relatively low impedance, resulting in excessive current flows.

When a fault occurs, both an ac symmetrical component of fault current and an asymmetrical dc transient component of fault current are created. The level of the dc component of current depends on the instantaneous current in each phase at the instance of the fault. The ac symmetrical component of current starts out very high, and decays to a steady-state level in two stages. The first period of very rapid decay is called the subtransient period and it lasts only a few cycles. The subtransient currents are basically associated with the time constants of the amortisseur or damper windings of generators and motors. The second period of slower decay is called the transient period and it lasts for 0.5 to 1.0 second. The transient currents are basically associated with the time constants of the field windings of generators and motors.

The reactances of generators and motors in the subtransient period are called subtransient reactances $\left(X^{\prime \prime}\right)$. The reactances of generators and motors in the transient period are called transient reactances $\left(X^{\prime}\right)$. The reactances of generators and motors in the steady-state period are the machines' synchronous reactances $\left(X_{S}\right)$. The resistances and reactances of transmission lines, transformers, and other static components do not change during the fault transients.

Fault currents can be calculated from the bus impedance matrix $\mathbf{Z}_{\text {bus }}$. A bus admittance matrix $\mathbf{Y}_{\text {bus }}$ is calculated separately for the subtransient, transient, and steady-state periods by using the appropriate reactances, and then inverted to obtain the bus impedance matrix $\mathbf{Z}_{\text {bus }}$. Once the bus impedance matrix $\mathbf{Z}_{\text {bus }}$ has been calculated, the fault current $\mathbf{I}_{f}$ at a particular bus $i$ can be found by dividing the prefault voltage $\mathbf{V}_{f}$ at the bus by the self-impedance (or driving point impedance) of the bus.

$$
\mathbf{I}_{f, i}^{\prime \prime}=\frac{\mathbf{V}_{f}}{Z_{i i}}(\mathrm{pu})
$$

If the subtransient bus impedance matrix $\mathbf{Z}_{\text {bus }}$ is used, the fault current will be the subtransient fault current $\mathbf{I}_{f}^{\prime \prime}$. Similarly, if the transient bus impedance matrix $\mathbf{Z}_{\mathrm{bus}}$ is used, the fault current will be the transient fault current $\mathbf{I}_{f}^{\prime}$.

If it is assumed that the power system was unloaded before the fault occurred, then the voltage at each bus can be calculated from the equation 


$$
\mathbf{V}_{j}=\left(1-\frac{Z_{j i}}{Z_{i i}}\right) \mathbf{V}_{f}(\mathrm{pu})
$$

where bus $i$ is the faulted bus. 


\section{Chapter 3 - Symmetrical Components and Unsymmetrical Faults}

\subsection{Introduction}

Most faults that occur on power systems are unsymmetrical faults [1], in which the magnitude and phase displacement between any two phases of voltages and currents in the three phases of the power system will differ. The most common example of an unsymmetrical fault is the single line-to-ground fault, in which there are high currents in one phase and the ground (neutral) only. Other examples include line-to-line faults and double line-to-ground faults. In all three cases, the magnitude and phase displacement between any two phases of voltages and currents differ for different phases of the power system. This presents a challenge, due to that the analysis technique used to study power systems during symmetrical three-phase faults assumes balanced fault conditions, i.e., the magnitude and phase displacement between any two phases of voltages and currents are identical in each phase of the power system. However, in this section a technique called the method of symmetrical components will be discussed, which will enable the analysis of unbalanced power systems and lead to accurate predictions of power system behaviour during unsymmetrical faults.

\subsection{Symmetrical Components}

In 1918, C.L. Fortescue introduced a technique for dealing with unbalanced polyphase systems called the method of symmetrical components [17]. Fortescue's work proves that an unbalanced system of $n$ related phasors can be resolved into $n$ systems of balanced phasors called the symmetrical components of the original phasors. The $n$ phasors of each set of components are equal in magnitude and the phase displacement between adjacent phasors of the set are equal. Although the method is applicable to any unbalanced polyphase system, the present discussion will be confined to unbalanced three-phase power systems. According to Fortescue's theorem, three unbalanced phasors of a three-phase power system can be resolved into three balanced systems of phasors. The balanced sets of components are:

1. A set of positive-sequence components consisting of three phasors equal in magnitude, displaced from each other by $120^{\circ}$ in phase, and having the same phase sequence as the original phasors.

2. A set of negative-sequence components consisting of three phasors equal in magnitude, displaced from each other by $120^{\circ}$ in phase, and having the opposite phase sequence from the original phasors.

3. A set of zero-sequence components consisting of three phasors equal in magnitude and phase (i.e., zero phase displacement from each other). 
It is customary when solving a problem by symmetrical components to designate the three phases of the power system as $a, b$, and $c$ in such a manner that the three phases peak in that order and the phase sequence of the voltages and currents in the power system is $a b c$. In such a power system, the positivesequence components will have phase sequence $a b c$, while the negative-sequence components will have phase sequence $a c b$. The zero-sequence components do not have a phase sequence, since all three phases peak at the same time. Traditionally, the positive-sequence components of the original phasors are designated with the subscript 1 , the negative-sequence components are designated with the subscript 2 , and the zero-sequence components are designated with the subscript 0 (as shown below in Figure 3.1). If the original phasors are the voltages in each phase of the power system and are designated $\mathbf{V}_{A}, \mathbf{V}_{B}$, and $\mathbf{V}_{C}$, then the positive-sequence components of the voltages will be $\mathbf{V}_{A 1}, \mathbf{V}_{B 1}$, and $\mathbf{V}_{C 1}$; the negativesequence components of the voltages will be $\mathbf{V}_{A 2}, \mathbf{V}_{B 2}$, and $\mathbf{V}_{C 2}$; and the zero-sequence components of the voltages will be $\mathbf{V}_{A 0}, \mathbf{V}_{B 0}$, and $\mathbf{V}_{C 0}$. The total voltage in each phase will be the sum of the three components for that phase.

$$
\begin{aligned}
& \mathbf{V}_{A}=\mathbf{V}_{A 1}+\mathbf{V}_{A 2}+\mathbf{V}_{A 0} \\
& \mathbf{V}_{B}=\mathbf{V}_{B 1}+\mathbf{V}_{B 2}+\mathbf{V}_{B 0} \\
& \mathbf{V}_{C}=\mathbf{v}_{C 1}+\mathbf{V}_{C 2}+\mathbf{V}_{C 0}
\end{aligned}
$$

The synthesis of a set of three unbalanced phasors from the three sets of symmetrical components of Figure 3.1 is shown on the next page in Figure 3.2.

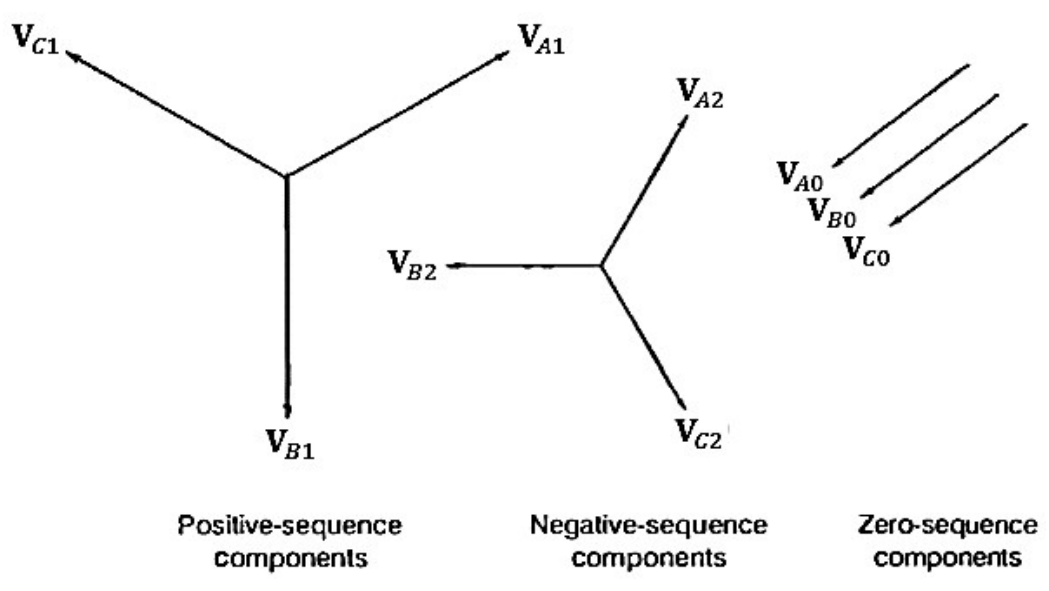

Figure 3.1: Three sets of balanced phasors which are the symmetrical components of three unbalanced phasors [1]. 


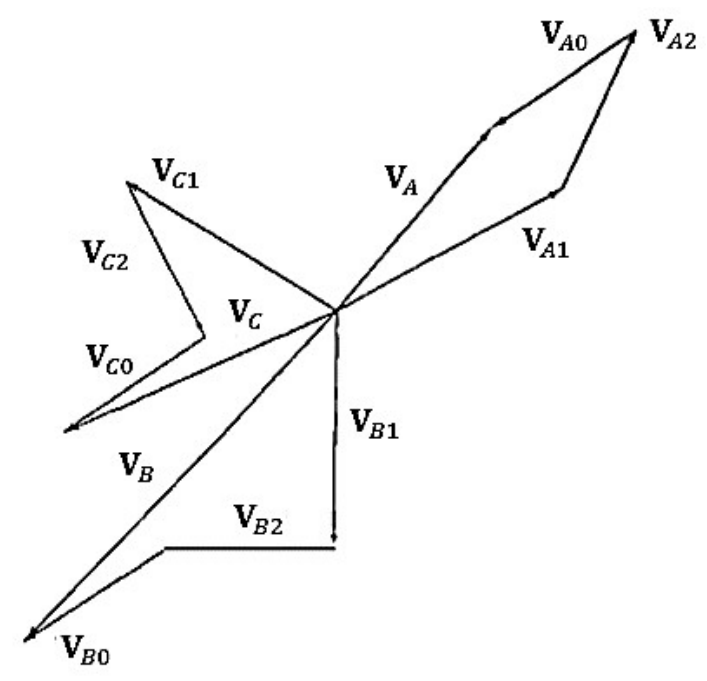

Figure 3.2: Graphical addition of the components shown in Figure 3.1 to obtain three unbalanced phasors [1].

In working with the symmetrical components of an unbalanced three-phase power system, the operation of shifting a phasor through an angle of $120^{\circ}$ occurs repeatedly. This operation is equivalent to multiplying the phasor by the quantity $1 \angle 120^{\circ}$. Since multiplication by this quantity occurs so often, the constant $a$ has been introduced to represent it such that each multiplication by $a$ rotates a phasor by $120^{\circ}$ without changing its magnitude. Therefore,

$$
\begin{gathered}
a=1 \angle 120^{\circ} \\
a^{2}=1 \angle 240^{\circ} \\
a^{3}=1 \angle 360^{\circ}=1 \angle 0^{\circ}=1
\end{gathered}
$$

Figure 3.3 below illustrates the relationships among the positive and negative exponents of $a$.

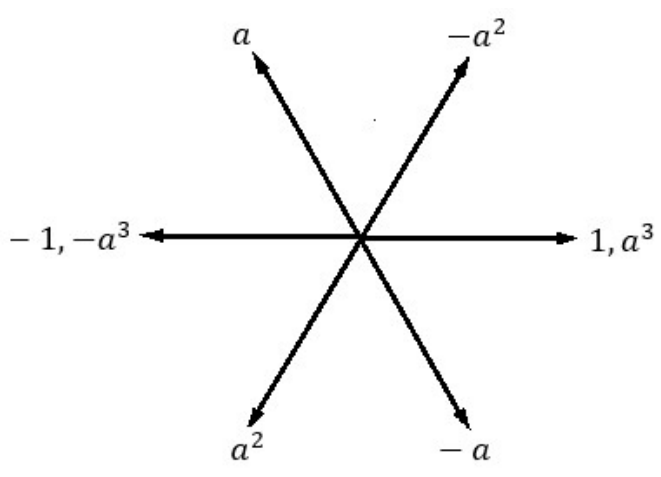

Figure 3.3: Phasor diagram illustrating the relationships among the various exponents of $a$ [3]. 
The symmetrical components of an unbalanced three-phase voltage can now be represented in terms of the $a$ constant, thereby reducing the number of unknown quantities by expressing each component of $\mathbf{V}_{B}$ and $\mathbf{V}_{C}$ as the product of a component of $\mathbf{V}_{A}$ and some function of the operator $a=1 \angle 120^{\circ}$. As mentioned earlier, the positive-sequence components have phase sequence $a b c$ (as shown previously in Figure 3.1). Therefore, the relationships among the positive-sequence components will be

$$
\begin{aligned}
& \mathbf{V}_{B 1}=a^{2} \mathbf{V}_{A 1} \\
& \mathbf{V}_{C 1}=a \mathbf{V}_{A 1}
\end{aligned}
$$

The negative-sequence components have phase sequence $a c b$ (as shown previously in Figure 3.1). Therefore, the relationships among the negative-sequence components will be

$$
\begin{aligned}
& \mathbf{V}_{B 2}=a \mathbf{V}_{A 2} \\
& \mathbf{V}_{C 2}=a^{2} \mathbf{V}_{A 2}
\end{aligned}
$$

The zero-sequence components are the same in all phases (as shown previously in Figure 3.1). Therefore, the relationships among the zero-sequence components will be

$$
\begin{aligned}
& \mathbf{V}_{B 0}=\mathbf{V}_{A 0} \\
& \mathbf{V}_{C 0}=\mathbf{V}_{A 0}
\end{aligned}
$$

It is now possible to determine the symmetrical components of an unbalanced three-phase voltage by resolving the three unbalanced phasors into their symmetrical components. The unbalanced set of threephase voltages is given in terms of symmetrical components by Equations (3.1), and the relationships between the phases of the positive-, negative-, and zero-sequence components is given by Equations (3.5) to (3.7). Substituting Equations (3.5) to (3.7) into Equations (3.1) yields

$$
\begin{aligned}
& \mathbf{V}_{A}=\mathbf{V}_{A 1}+\mathbf{V}_{A 2}+\mathbf{V}_{A 0} \\
& \mathbf{V}_{B}=a^{2} \mathbf{V}_{A 1}+a \mathbf{V}_{A 2}+\mathbf{V}_{A 0} \\
& \mathbf{V}_{C}=a \mathbf{V}_{A 1}+a^{2} \mathbf{V}_{A 2}+\mathbf{V}_{A 0}
\end{aligned}
$$

In matrix form, Equations (3.8) to (3.10) become

$$
\left[\begin{array}{l}
\mathbf{V}_{A} \\
\mathbf{V}_{B} \\
\mathbf{V}_{C}
\end{array}\right]=\left[\begin{array}{ccc}
1 & 1 & 1 \\
1 & a^{2} & a \\
1 & a & a^{2}
\end{array}\right]\left[\begin{array}{l}
\mathbf{V}_{A 0} \\
\mathbf{V}_{A 1} \\
\mathbf{V}_{A 2}
\end{array}\right]=A\left[\begin{array}{l}
\mathbf{V}_{A 0} \\
\mathbf{V}_{A 1} \\
\mathbf{V}_{A 2}
\end{array}\right]
$$


where

$$
A=\left[\begin{array}{ccc}
1 & 1 & 1 \\
1 & a^{2} & a \\
1 & a & a^{2}
\end{array}\right]
$$

Therefore, the symmetrical components of the unbalanced three-phase voltage can be expressed as

$$
\left[\begin{array}{c}
\mathbf{V}_{A 0} \\
\mathbf{V}_{A 1} \\
\mathbf{V}_{A 2}
\end{array}\right]=A^{-1}\left[\begin{array}{c}
\mathbf{V}_{A} \\
\mathbf{V}_{B} \\
\mathbf{V}_{C}
\end{array}\right]
$$

Since

$$
A^{-1}=\left[\begin{array}{ccc}
1 & 1 & 1 \\
1 & a^{2} & a \\
1 & a & a^{2}
\end{array}\right]^{-1}=\frac{1}{3}\left[\begin{array}{ccc}
1 & 1 & 1 \\
1 & a & a^{2} \\
1 & a^{2} & a
\end{array}\right]
$$

Equation (3.13) becomes

$$
\left[\begin{array}{l}
\mathbf{V}_{A 0} \\
\mathbf{V}_{A 1} \\
\mathbf{V}_{A 2}
\end{array}\right]=\frac{1}{3}\left[\begin{array}{ccc}
1 & 1 & 1 \\
1 & a & a^{2} \\
1 & a^{2} & a
\end{array}\right]\left[\begin{array}{l}
\mathbf{V}_{A} \\
\mathbf{V}_{B} \\
\mathbf{V}_{C}
\end{array}\right]
$$

Equation (3.15) shows how to calculate the symmetrical components of phase $a$ of an unbalanced threephase voltage. In expanded form, Equation (3.15) becomes

$$
\begin{array}{ll}
\mathbf{V}_{A 0}=\frac{1}{3}\left(\mathbf{V}_{A}+\mathbf{V}_{B}+\mathbf{V}_{C}\right) & \text { zero-sequence } \\
\mathbf{V}_{A 1}=\frac{1}{3}\left(\mathbf{V}_{A}+a \mathbf{V}_{B}+a^{2} \mathbf{V}_{C}\right) & \text { positive-sequence } \\
\mathbf{V}_{A 2}=\frac{1}{3}\left(\mathbf{V}_{A}+a^{2} \mathbf{V}_{B}+a \mathbf{V}_{C}\right) & \text { negative-sequence }
\end{array}
$$

The symmetrical components of the other two phases can be calculated from Equations (3.5) to (3.7). Similar results apply to line-to-line voltages simply by replacing $\mathbf{V}_{A}, \mathbf{V}_{B}$, and $\mathbf{V}_{C}$ in Equations (3.16) to (3.18) by $\mathbf{V}_{A B}, \mathbf{V}_{B C}$, and $\mathbf{V}_{C A}$, respectively.

Equation (3.16) shows that no zero-sequence components exist if the sum of the three unbalanced phasors is zero. Since the sum of the line-to-line voltage phasors in a three-phase power system is always zero, zero-sequence components are never present in the line-to-line voltages regardless of the degree of 
unbalance. The sum of the three line-to-neutral voltage phasors is not necessarily zero, and hence line-toneutral voltages may contain zero-sequence components.

The preceding equations could have been written for any set of related phasors, and the relationships among unbalanced three-phase currents have the same forms as the relationships among unbalanced three-phase voltages. The currents in each phase can be represented in terms of symmetrical components as

$$
\begin{aligned}
& \mathbf{I}_{A}=\mathbf{I}_{A 1}+\mathbf{I}_{A 2}+\mathbf{I}_{A 0} \\
& \mathbf{I}_{B}=\mathbf{I}_{B 1}+\mathbf{I}_{B 2}+\mathbf{I}_{B 0} \\
& \mathbf{I}_{C}=\mathbf{I}_{C 1}+\mathbf{I}_{C 2}+\mathbf{I}_{C 0}
\end{aligned}
$$

Substituting the relationships between the phases of the positive-, negative-, and zero-sequence components yields

$$
\begin{aligned}
& \mathbf{I}_{A}=\mathbf{I}_{A 1}+\mathbf{I}_{A 2}+\mathbf{I}_{A 0} \\
& \mathbf{I}_{B}=a^{2} \mathbf{I}_{A 1}+a \mathbf{I}_{A 2}+\mathbf{I}_{A 0} \\
& \mathbf{I}_{C}=a \mathbf{I}_{A 1}+a^{2} \mathbf{I}_{A 2}+\mathbf{I}_{A 0}
\end{aligned}
$$

and the symmetrical components can be represented in terms of the currents in each phase as

$$
\begin{array}{ll}
\mathbf{I}_{A 0}=\frac{1}{3}\left(\mathbf{I}_{A}+\mathbf{I}_{B}+\mathbf{I}_{C}\right) & \text { zero-sequence } \\
\mathbf{I}_{A 1}=\frac{1}{3}\left(\mathbf{I}_{A}+a \mathbf{I}_{B}+a^{2} \mathbf{I}_{C}\right) & \text { positive-sequence } \\
\mathbf{I}_{A 2}=\frac{1}{3}\left(\mathbf{I}_{A}+a^{2} \mathbf{I}_{B}+a \mathbf{I}_{C}\right) & \text { negative-sequence }
\end{array}
$$

Equation (3.25) provides an important insight into the operation of unbalanced three-phase power systems. Comparing the current in the neutral of a power system given by $\mathbf{I}_{N}=\mathbf{I}_{A}+\mathbf{I}_{B}+\mathbf{I}_{C}$ to Equation (3.25) reveals that

$$
\mathbf{I}_{N}=3 \mathbf{I}_{A 0}
$$

The current in the neutral (or ground) of the power system is equal to 3 times the zero-sequence component of current. Therefore, if a system element has no neutral current, then there can be no zero-sequence components of currents in the element. Hence $\Delta$-connected and ungrounded $Y$-connected elements cannot have zero-sequence components of currents. 


\subsection{Sequence Impedances and Sequence Networks}

In any part of the power system, the voltage drop caused by the current of a particular sequence depends on only the impedance of that part of the power system to current flow of that sequence [1]. In general, the impedance of a circuit can differ for positive-, negative-, and zero-sequence currents. The impedance of a circuit to positive-sequence current is called the positive-sequence impedance of the circuit. Similarly, the impedance of the circuit to negative-sequence current is called the negative-sequence impedance of the circuit, and the impedance of the circuit to zero-sequence current is called the zero-sequence impedance of the circuit. Due to that currents of a particular sequence produce voltages drops of the same sequence

only, it is possible to create separate positive-, negative-, and zero-sequence per-unit equivalent circuits called sequence networks. The overall behaviour of a power system during unsymmetrical faults can then be obtained by treating each set of symmetrical components separately and superimposing the results.

As mentioned earlier, in a three-phase power system which is normally balanced, unbalanced fault conditions generally cause unbalanced voltages and currents to exist in each of the phases. If the voltages and currents are related by constant impedances, the system is said to be linear and the principle of superposition applies. The voltage response of the linear system to the unbalanced currents can be determined by considering the separate responses of each system element to the symmetrical components of the currents and superimposing the results. For the present discussion, the system elements of interest are the machines, transmission lines, and loads connected in $\Delta$ or $Y$ configurations, although a simplifying assumption typically made in industry-based fault studies are that load impedances are much larger than those of network components, and thus can be neglected in system modelling [1].

Since the response of each system element depends on the symmetrical component of current being considered, three different per-phase equivalent circuits must be constructed for each element of the threephase power system, one for each type of symmetrical component to reflect the separate responses of the system elements to the currents of each sequence. The positive-sequence network is a per-phase equivalent circuit containing only the positive-sequence impedances and sources, which means that it is identical to the per-phase equivalent circuit used for symmetrical fault analysis covered in the previous section. The negative-sequence network is a per-phase equivalent circuit containing only the negativesequence impedances, and the zero-sequence network is a per-phase equivalent circuit containing only the zero-sequence impedances. Once the positive-, negative-, and zero-sequence networks are constructed, they are interconnected according to the type of fault being analyzed, and the positive-, negative, and zero-sequence components of current are calculated. These components can then be combined by using Equations (3.22) to (3.24) to calculate the unbalanced three-phase currents flowing in the fault and elsewhere within the power system. 


\subsection{Positive-, Negative-, and Zero-Sequence Equivalent Circuits of Generators}

The equivalent circuit of a Y-connected, three-phase synchronous generator grounded through an inductive reactance is shown on page 33 in Figure 3.4. The synchronous generator as seen by positive-sequence currents is shown on page 34 in Figure 3.5(a), together with the positive-sequence equivalent circuit for the generator shown in Figure 3.5(b). Note that it is the same as the per-phase equivalent circuit used in the previous section for symmetrical three-phase fault analysis. The synchronous generator is modeled by an internal generated voltage in series with the inductive reactance of each winding in the generator to positivesequence current, denoted by the positive-sequence impedance $Z_{1}$. For subtransient fault analysis, the reactance is the subtransient reactance $X^{\prime \prime}$. For transient fault analysis, the reactance is the transient reactance $X^{\prime}$. For steady-state fault analysis, the reactance is the machine's synchronous reactance $X_{S}$.

Similarly, the synchronous generator as seen by negative-sequence currents is shown on page 34 in Figure 3.5(c), together with the negative-sequence equivalent circuit for the generator shown in Figure 3.5(d). The negative-sequence equivalent circuit does not contain voltage sources because there are no voltage sources inside the generator that generate negative-sequence voltages. Therefore, the synchronous generator is modeled by the inductive reactance of each winding in the generator to negative-sequence current, denoted by the negative-sequence impedance $Z_{2}$. As mentioned earlier, the negative-sequence currents have the opposite phase sequence to the positive-sequence currents and by changing the phase sequence of the currents applied to a three-phase stator, the direction of rotation of the magnetic fields produced in the machine becomes reversed. As a result, the negative-sequence currents produce a rotating magnetic field opposite to the direction of rotation of the machine [3]. This backward rotation induces a very high voltage in the amortisseur or damper windings of the generator and because the magnetic fields continue to turn opposite to the rotation of the generator, this voltage doesn't decay after a few cycles. Therefore, the negative-sequence currents see a reactance that is approximately the size of the subtransient reactance of the generator at all times.

Finally, the synchronous generator as seen by zero-sequence currents is shown on page 34 in Figure 3.5(e), together with the zero-sequence equivalent circuit for the generator shown in Figure 3.5(f). The zerosequence equivalent circuit does not contain voltage sources because there are no voltage sources inside the generator that generate zero-sequence voltages. Therefore, the synchronous generator is modeled by the inductive reactance of each winding in the generator to zero-sequence current, denoted by the zerosequence impedance $Z_{g 0}$. The total current flowing in the impedance $Z_{N}$ between the neutral and the ground of the generator is $3 \mathbf{I}_{A 0}$ because the zero-sequence current from all three phases flows through it and the current is identical in each phase. Therefore, the voltage drop from point $a$ to ground will be $-3 \mathbf{I}_{A 0} Z_{N}-\mathbf{I}_{A 0} Z_{g 0}$. To represent this voltage drop in the per-phase equivalent circuit, which contains only 
the zero-sequence current in phase $a$, an impedance 3 times larger than the physical impedance between the neutral and the ground of the generator must be used. The total zero-sequence impedance of the generator is

$$
Z_{0}=Z_{g 0}+3 Z_{N}
$$

The zero-sequence impedance $Z_{g 0}$ of each winding in the generator consists of only a relatively small inductive reactance due to that the zero-sequence currents in all three phases increase and decrease together, causing their magnetic fields to cancel each other out and produce only a small reactance. The zero-sequence reactance of a generator's windings can be as small as one-quarter the machine's subtransient reactance [3].

The positive-, negative-, and zero-sequence voltages in phase $a$ of the generator can be found by applying Kirchhoff's voltage law to each of the per-phase equivalent circuits, which leads to

$$
\begin{aligned}
\mathbf{v}_{A 1} & =\mathbf{E}_{A 1}-\mathbf{I}_{A 1} Z_{1} \\
\mathbf{v}_{A 2} & =-\mathbf{I}_{A 2} Z_{2} \\
\mathbf{v}_{A 0} & =-\mathbf{I}_{A 0} Z_{0}
\end{aligned}
$$

where $\mathbf{E}_{A 1}$ is the positive-sequence internal generated voltage of the generator, and the impedances $Z_{1}$, $Z_{2}$, and $Z_{0}$ are the positive-, negative-, and zero-sequence impedances of the generator, respectively. Note that the discussion above can be applied equally to synchronous motors and synchronous generators, since they are physically the same machine. 


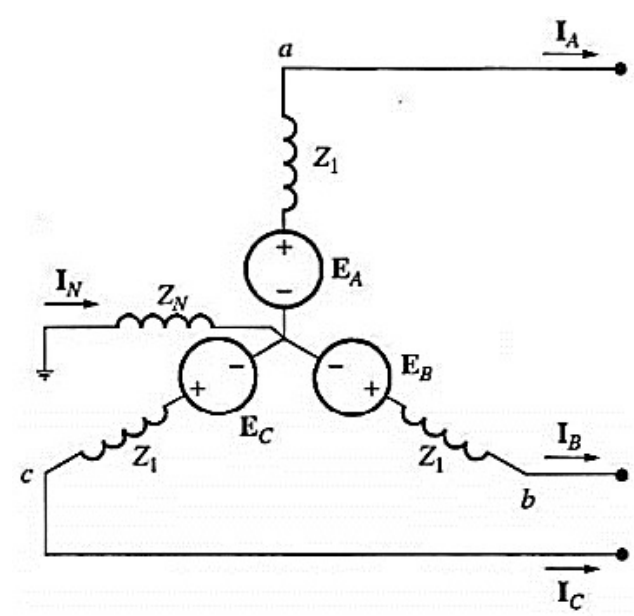

Figure 3.4: A Y-connected three-phase generator grounded through an inductive reactance [3]. 

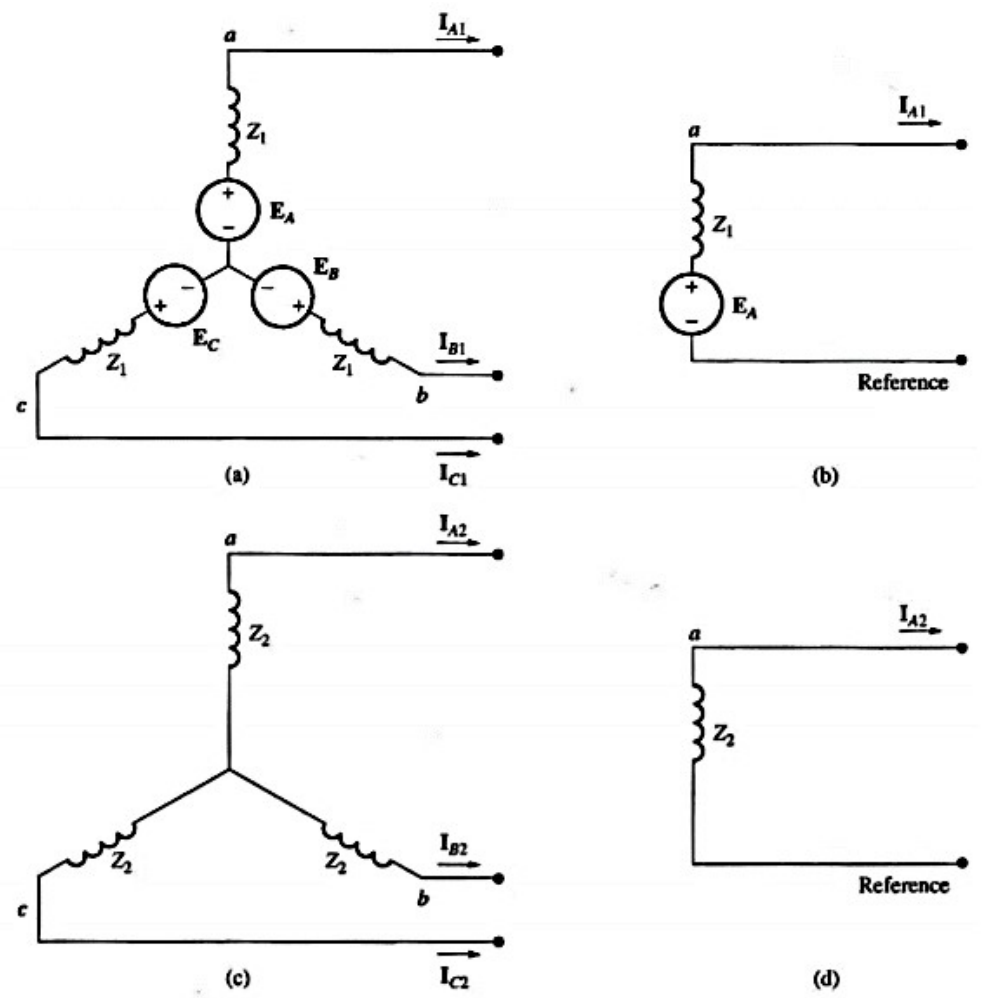

(b)
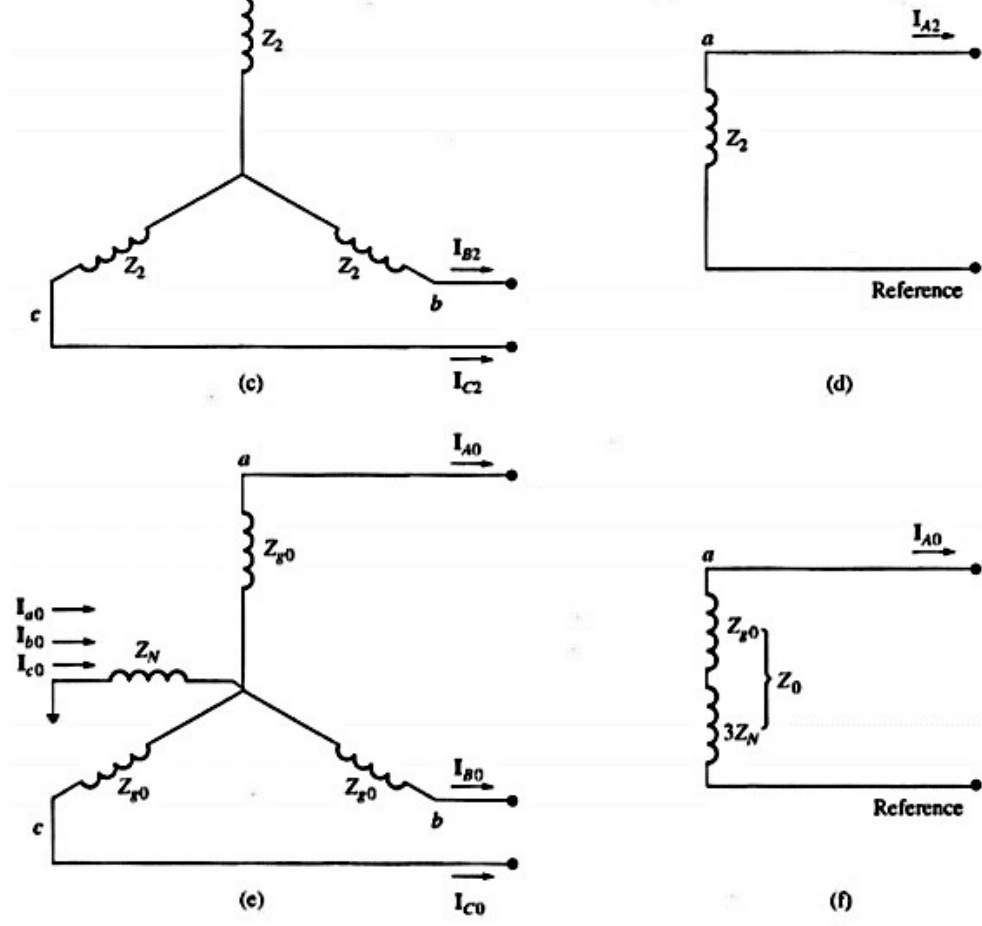

(d)

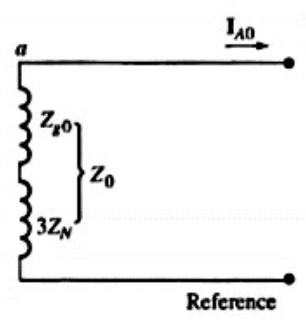

(f)

Figure 3.5: (a) A synchronous generator as seen by positive-sequence currents. (b) The positive-sequence equivalent circuit for the generator. (c) A synchronous generator as seen by negative-sequence currents. (d) The negativesequence equivalent circuit for the generator. (e) A synchronous generator as seen by zero-sequence currents. (f) The zero-sequence equivalent circuit for the generator [3]. 


\subsection{Positive-, Negative-, and Zero-Sequence Equivalent Circuits of Transmission Lines}

Due to that transmission lines consist of static components, there is no difference between how they respond to positive- and negative-sequence currents. Therefore, the equivalent circuit for a transmission line is identical for both positive- and negative-sequence networks. This equivalent circuit consists of simply the series impedance of the transmission line. However, overhead transmission lines respond very differently for zero-sequence currents compared to positive- and negative-sequence currents. The reason being the series inductance of a transmission line increases in direct proportion to the natural logarithm of the distance between the sending and returning conductors [3]. For zero-sequence currents, all three phases of the transmission line carry equal currents, and the return path is either through overhead ground wires or through the ground itself. Since both the ground wires and the ground itself are typically well separated from the phases, the zero-sequence inductive reactance of a transmission line is generally larger than the positive- and negative-sequence inductive reactance. For transmission lines without overhead ground wires, the return path must be through the ground itself, and the zero-sequence inductive reactance will be even larger because the phases are so far above the ground. The zero-sequence inductive reactance of an overhead transmission line will be 2 to 3.5 times larger than the positive- or negativesequence inductive reactance, with overhead transmission lines without overhead ground wires at the upper end of this range [1]. Note that the series resistance of the transmission line, which is often neglected as a simplifying assumption made in creating per-phase equivalent circuits for fault current analysis, remains the same for all three symmetrical components of current. Although the discussion thus far has primarily focused on power systems that are normally balanced and only become unbalanced upon the occurrence of an unsymmetrical fault, in practical transmission systems such complete symmetry is more ideal than realized, but since the effect of the departure from symmetry is typically small, perfect balance between phases is often assumed especially if the transmission lines are transposed along their lengths [1]. 


\subsection{Positive-, Negative-, and Zero-Sequence Equivalent Circuits of $\mathrm{Y}$ and $\Delta$ Connections}

Due to that $Y$ - and $\Delta$-connected loads are static devices, there is no difference between how they respond to positive- and negative-sequence currents. Therefore, the equivalent circuit for a $\mathrm{Y}$ - or $\Delta$-connected load is identical for both positive- and negative-sequence networks. This equivalent circuit consists of simply the $\mathrm{Y}$ - or $\Delta$-connected impedance of the load. However, $\mathrm{Y}$ - and $\Delta$-connected loads respond very differently for zero-sequence currents compared to positive- and negative-sequence currents. As mentioned earlier, zerosequence currents have the same phase in all three phases of a three-phase power system, thus they can only flow if there is a path to the ground for the return current. If there is no return path, then no zerosequence currents can flow.

An ungrounded Y-connected load is shown on the next page in Figure 3.6(a). Due to that there is no path between the neutral and the ground in this load, no zero-sequence currents can flow. The corresponding zero-sequence equivalent circuit has an impedance $Z$ between the terminals of the load and the neutral, and an open-circuit between the neutral and the ground.

A grounded Y-connected load is shown on the next page in Figure 3.6(b). Due to that there is a path between the neutral and the ground in this load, zero-sequence currents can flow. The corresponding zerosequence equivalent circuit has an impedance $Z$ between the terminals of the load and the neutral, and a short-circuit between the neutral and the ground.

A Y-connected load that is grounded through an impedance is shown on the next page in Figure 3.6(c). Due to that there is a path between the neutral and the ground in this load, zero-sequence currents can flow. However, the current flowing in the path between the neutral and the ground is 3 times the zerosequence current in any single phase, thus the corresponding impedance in the zero-sequence equivalent circuit is $3 Z_{N}$. The zero-sequence equivalent circuit has an impedance $Z$ between the terminals of the load and the neutral, and an impedance $3 Z_{N}$ between the neutral and the ground.

A $\Delta$-connected load is shown on the next page in Figure 3.6(d). Due to that there is no path to ground in this load, no zero-sequence currents can flow into the load. However, any zero-sequence currents inside the load can flow freely within the $\Delta$ because the phases of the $\Delta$-connected load are connected head to tail in a closed loop. The corresponding zero-sequence equivalent circuit has an open-circuit at the terminals, and a closed loop containing the impedance $Z$. 

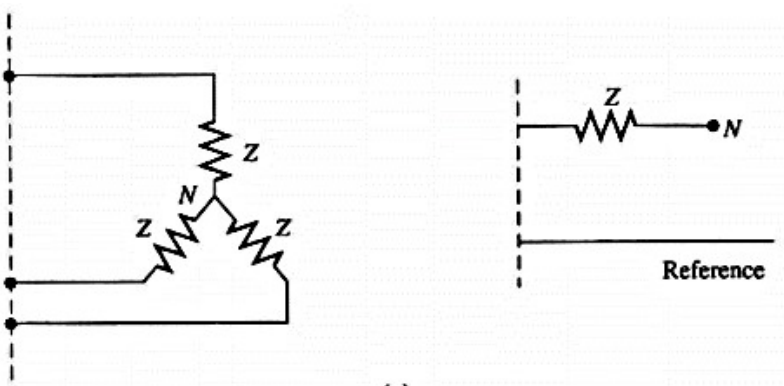

(a)
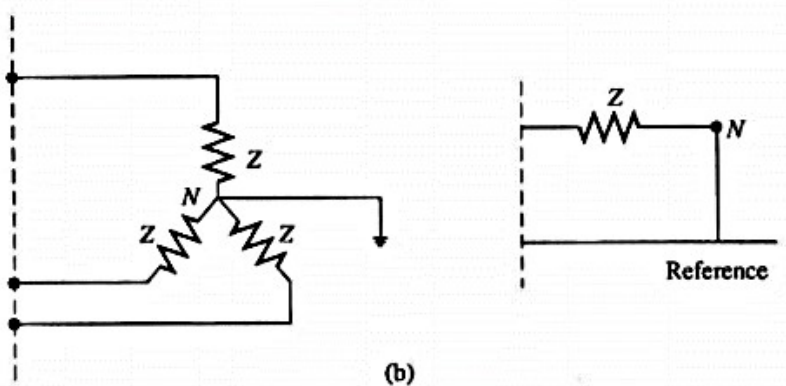

(b)
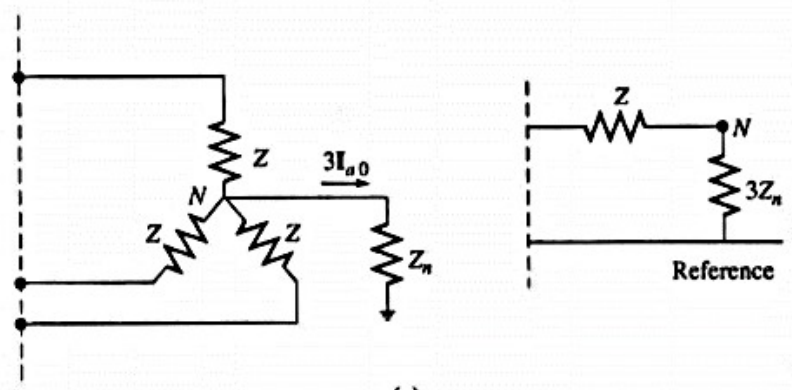

(c)
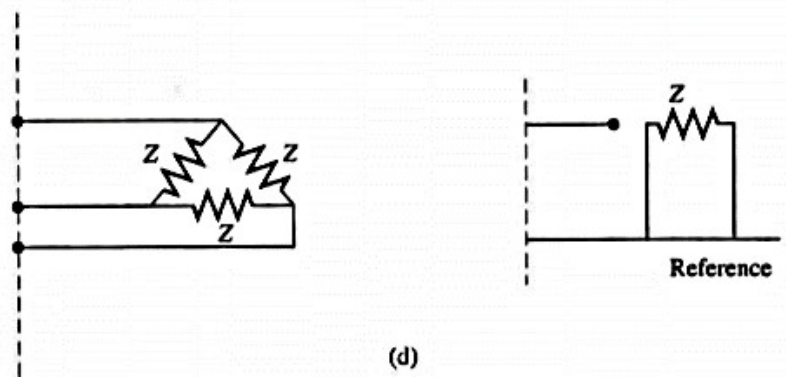

(d)

Figure 3.6: (a) An ungrounded Y-connected load has no path between the neutral and the ground, thus the zerosequence equivalent circuit has an open-circuit between the neutral and the ground. (b) A grounded Y-connected load has a short-circuit between the neutral and the ground, thus the zero-sequence equivalent circuit has a short-circuit between the neutral and the ground. (c) A Y-connected load grounded through an impedance. The zero-sequence equivalent circuit has the impedance $3 Z_{N}$ between the neutral and the ground. (d) $\mathrm{A} \Delta$-connected load has no path to the ground, thus no zero-sequence currents can flow into the load. However, any zero-sequence currents inside the load can flow freely within the $\Delta[3]$. 


\subsection{Positive-, Negative-, and Zero-Sequence Equivalent Circuits of Transformers}

Due to that transformers are static devices, there is no difference between how they respond to positiveand negative-sequence currents. Therefore, the equivalent circuit for a transformer is identical for both positive- and negative-sequence networks. This equivalent circuit consists of simply the series impedance of the transformer. Note that the shunt components representing the excitation branch of the transformer are typically neglected as a simplifying assumption made in creating per-phase equivalent circuits for fault current analysis [3]. However, transformers respond very differently for zero-sequence currents compared to positive- and negative-sequence currents. Each side of a two-winding transformer is either Y-connected or $\Delta$-connected, thus the zero-sequence equivalent circuit of a side will appear as one of the $\mathrm{Y}-$ or $\Delta$ connected zero-sequence equivalent circuits shown previously in Figure 3.6. However, the current flow on each side of a two-winding transformer is related by the turns ratio of the transformer, i.e., $N_{P} \mathbf{I}_{P}=N_{S} \mathbf{I}_{S}$. Therefore, if there is no path for zero-sequence currents on one side of the transformer, there must also be no zero-sequence currents on the other side of the transformer.

There are five possible cases of zero-sequence equivalent circuits for three-phase transformers depending on the connections of their primary and secondary windings as shown on the next page in Figure 3.7. 


\begin{tabular}{|l|l|l|l|}
\hline Symbol & Connection & $\begin{array}{c}\text { Zero-sequence } \\
\text { equivalent circuit }\end{array}$ \\
\hline & & \\
\hline
\end{tabular}

Figure 3.7: Symbols, connection diagrams, and zero-sequence equivalent circuits for various types of three-phase transformers [3]. 


\subsection{Single Line-to-Ground Fault on an Unloaded Generator}

An unloaded generator with a single line-to-ground fault on phase $a$ is shown on the next page in Figure 3.8. When a single line-to-ground fault occurs on phase $a$ of an unloaded generator, the voltage in phase $a$ at the location of the fault becomes zero, and the currents in phases $b$ and $c$ remain zero.

$$
\left.\begin{array}{rl}
\mathbf{I}_{B} & =0 \\
\mathbf{I}_{C} & =0 \\
\mathbf{V}_{A} & =0
\end{array}\right\} \text { single line-to-ground fault }
$$

The symmetrical components of current in the fault are given in terms of the currents in each phase by Equations (3.25) to (3.27). Substituting $\mathbf{I}_{B}=0$ and $\mathbf{I}_{C}=0$ into Equations (3.25) to (3.27) yields

$$
\begin{aligned}
& \mathbf{I}_{A 0}=\frac{1}{3}\left(\mathbf{I}_{A}+0+0\right)=\frac{1}{3} \mathbf{I}_{A} \\
& \mathbf{I}_{A 1}=\frac{1}{3}\left[\mathbf{I}_{A}+a(0)+a^{2}(0)\right]=\frac{1}{3} \mathbf{I}_{A} \\
& \mathbf{I}_{A 2}=\frac{1}{3}\left[\mathbf{I}_{A}+a^{2}(0)+a(0)\right]=\frac{1}{3} \mathbf{I}_{A}
\end{aligned}
$$

Therefore,

$$
\mathbf{I}_{A 0}=\mathbf{I}_{A 1}=\mathbf{I}_{A 2}=\frac{1}{3} \mathbf{I}_{A}
$$

Equation (3.37) shows that the symmetrical components of current are all equal in a single line-to-ground fault. Substituting $\mathbf{I}_{A 2}=\mathbf{I}_{A 1}$ and $\mathbf{I}_{A 0}=\mathbf{I}_{A 1}$ into Equations (3.30) to (3.32) yields

$$
\begin{aligned}
\mathbf{V}_{A 1} & =\mathbf{E}_{A 1}-\mathbf{I}_{A 1} Z_{1} \\
\mathbf{V}_{A 2} & =-\mathbf{I}_{A 1} Z_{2} \\
\mathbf{V}_{A 0} & =-\mathbf{I}_{A 1} Z_{0}
\end{aligned}
$$

The voltage in phase $a$ is given in terms of symmetrical components by Equation (3.8). Substituting Equations (3.38) to (3.40) into Equation (3.8) yields

$$
\mathbf{V}_{A}=\mathbf{V}_{A 1}+\mathbf{V}_{A 2}+\mathbf{V}_{A 0}=\mathbf{E}_{A 1}-\mathbf{I}_{A 1} Z_{1}-\mathbf{I}_{A 1} Z_{2}-\mathbf{I}_{A 1} Z_{0}=0
$$

Rearranging Equation (3.41) to solve for the positive-sequence current in the fault yields

$$
\mathbf{I}_{A 1}=\frac{\mathbf{E}_{A}}{Z_{0}+Z_{1}+Z_{2}}
$$


For a single line-to-ground fault, the positive-, negative-, and zero-sequence current are all equal, and the total impedance is the series combination of $Z_{1}, Z_{2}$, and $Z_{0}$. Note that this result is equivalent to connecting the positive-, negative-, and zero-sequence networks in series at the terminals representing the location of the fault, as shown on the next page in Figure 3.9.

In summary, to calculate the voltages and currents in a single line-to-ground fault on a three-phase synchronous generator [3]:

1. Create the positive-, negative-, and zero-sequence networks for the generator.

2. Connect the sequence networks in series at the terminals representing the location of the fault.

3. Calculate $\mathbf{I}_{A 0}=\mathbf{I}_{A 1}=\mathbf{I}_{A 2}$ from this network.

4. Calculate $\mathbf{V}_{A 0}, \mathbf{V}_{A 1}$, and $\mathbf{V}_{A 2}$ from Equations (3.30) to (3.32).

5. Calculate any required voltages and currents by combining the symmetrical components calculated above using Equations (3.8) to (3.10) and (3.22) to (3.24).

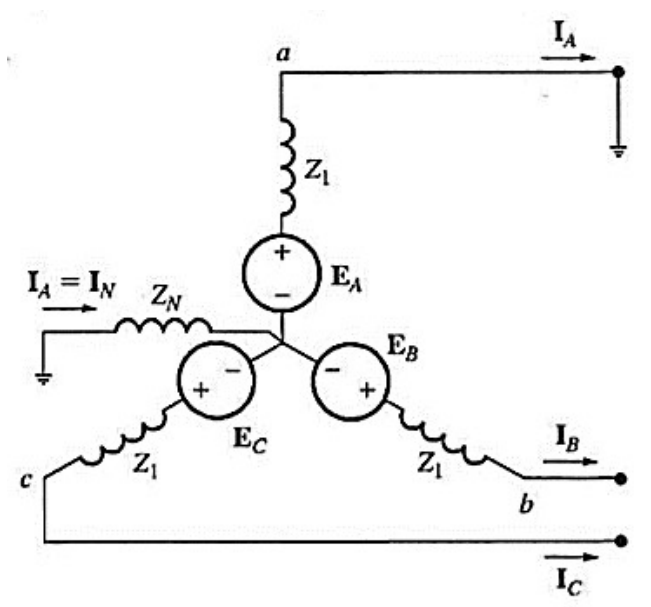

Figure 3.8: A single line-to-ground fault on phase $a$ of an unloaded generator. The generator's neutral is grounded through an inductive reactance [3]. 


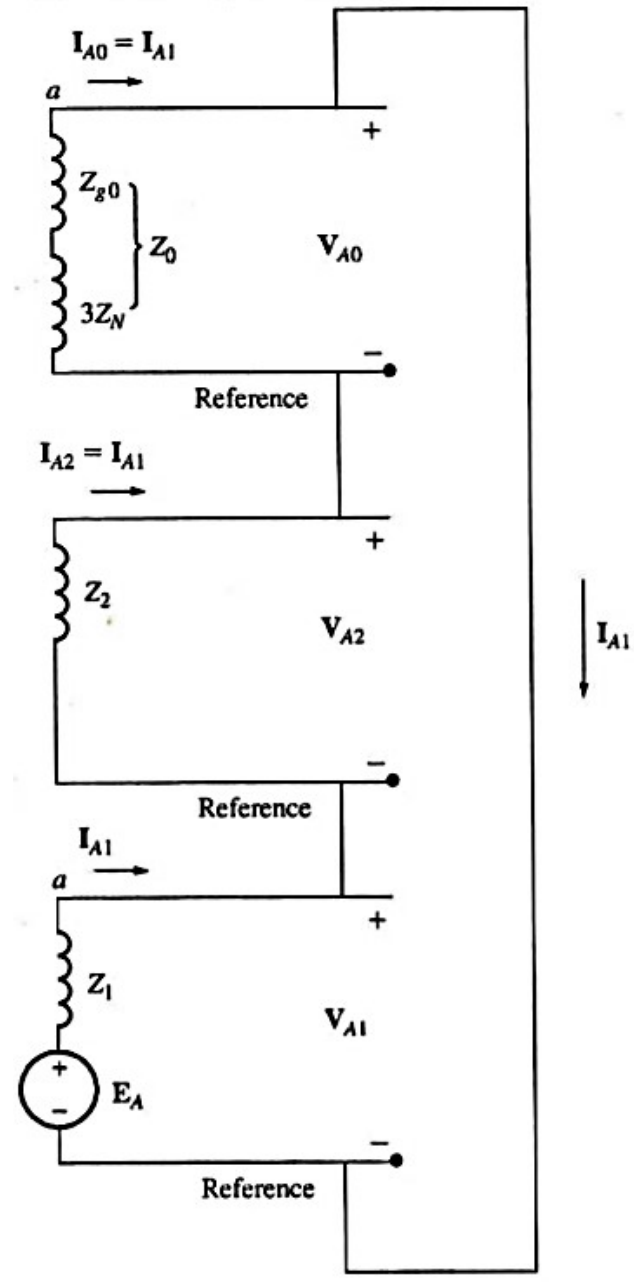

Figure 3.9: For a single line-to-ground fault, the positive-, negative-, and zero-sequence networks are connected in series to determine the sequence voltages and currents in the fault [3]. 


\subsection{Line-to-Line Fault on an Unloaded Generator}

An unloaded generator with a line-to-line fault between phases $b$ and $c$ is shown on the next page in Figure 3.10. When a line-to-line fault occurs between phases $b$ and $c$ of an unloaded generator, the voltages in phases $b$ and $c$ must be equal, and the currents in phases $b$ and $c$ must be the negative of each other.

$$
\left.\begin{array}{rl}
\mathbf{V}_{B} & =\mathbf{V}_{C} \\
\mathbf{I}_{A} & =0 \\
\mathbf{I}_{B} & =-\mathbf{I}_{C}
\end{array}\right\} \text { line-to-line fault }
$$

Substituting $\mathbf{V}_{C}=\mathbf{V}_{B}$ into Equations (3.16) to (3.18) yields

$$
\begin{aligned}
& \mathbf{V}_{A 0}=\frac{1}{3}\left(\mathbf{V}_{A}+\mathbf{V}_{B}+\mathbf{V}_{B}\right) \\
& \mathbf{V}_{A 1}=\frac{1}{3}\left(\mathbf{V}_{A}+a \mathbf{V}_{B}+a^{2} \mathbf{V}_{B}\right) \\
& \mathbf{V}_{A 2}=\frac{1}{3}\left(\mathbf{V}_{A}+a^{2} \mathbf{V}_{B}+a \mathbf{V}_{B}\right)
\end{aligned}
$$

Note that Equations (3.45) and (3.46) are identical, implying that for a line-to-line fault $\mathbf{V}_{A 1}=\mathbf{V}_{A 2}$. The symmetrical components of current in the fault are given in terms of the currents in each phase by Equations (3.25) to (3.27). Substituting $\mathbf{I}_{A}=0$ and $\mathbf{I}_{B}=-\mathbf{I}_{C}$ into Equations (3.25) to (3.27) yields

$$
\begin{aligned}
& \mathbf{I}_{A 0}=\frac{1}{3}\left(0-\mathbf{I}_{C}+\mathbf{I}_{C}\right) \\
& \mathbf{I}_{A 1}=\frac{1}{3}\left[0-a \mathbf{I}_{C}+a^{2} \mathbf{I}_{C}\right] \\
& \mathbf{I}_{A 2}=\frac{1}{3}\left[0-a^{2} \mathbf{I}_{C}+a \mathbf{I}_{C}\right]
\end{aligned}
$$

From Equations (3.47) to (3.49), it can readily be seen that $\mathbf{I}_{A 0}=0$ and $\mathbf{I}_{A 2}=-\mathbf{I}_{A 1}$. Substituting $\mathbf{V}_{A 1}=$ $\mathbf{V}_{A 2}, \mathbf{I}_{A 0}=0$, and $\mathbf{I}_{A 2}=-\mathbf{I}_{A 1}$ into Equations (3.30) to (3.32) yields

$$
\begin{aligned}
\mathbf{V}_{A 1} & =\mathbf{E}_{A 1}-\mathbf{I}_{A 1} Z_{1} \\
\mathbf{V}_{A 1} & =\mathbf{I}_{A 1} Z_{2} \\
\mathbf{V}_{A 0} & =-\mathbf{I}_{A 0} Z_{0}=0
\end{aligned}
$$

From Equations (3.50) and (3.51), it can be observed that

$$
\mathbf{I}_{A 1} Z_{2}=\mathbf{E}_{A 1}-\mathbf{I}_{A 1} Z_{1}
$$




$$
\mathbf{I}_{A 1}=\frac{\mathbf{E}_{A 1}}{Z_{1}+Z_{2}}
$$

For a line-to-line fault, the positive-sequence current is the negative of the negative-sequence current, and the zero-sequence current is zero (this makes sense physically because the fault is not connected to the ground, thus there is no path to ground for the zero-sequence currents to flow through). In addition, the positive-sequence phase voltage is equal to the negative-sequence phase voltage. Note that this result is equivalent to connecting the positive- and negative-sequence networks in parallel at the terminals representing the location of the fault, as shown on the next page in Figure 3.11.

In summary, to calculate the voltages and currents in a line-to-line fault on a three-phase synchronous generator [3]:

1. Create the positive- and negative-sequence networks for the generator. The zero-sequence network is not required.

2. Connect the positive- and negative-sequence networks in parallel at the terminals representing the location of the fault.

3. Calculate $\mathbf{I}_{A 1}=-\mathbf{I}_{A 2}$ from this network. Note that $\mathbf{I}_{A 0}=0$.

4. Calculate $\mathbf{V}_{A 0}, \mathbf{V}_{A 1}$, and $\mathbf{V}_{A 2}$ from Equations (3.30) to (3.32).

5. Calculate any required voltages and currents by combining the symmetrical components calculated above using Equations (3.8) to (3.10) and (3.22) to (3.24).

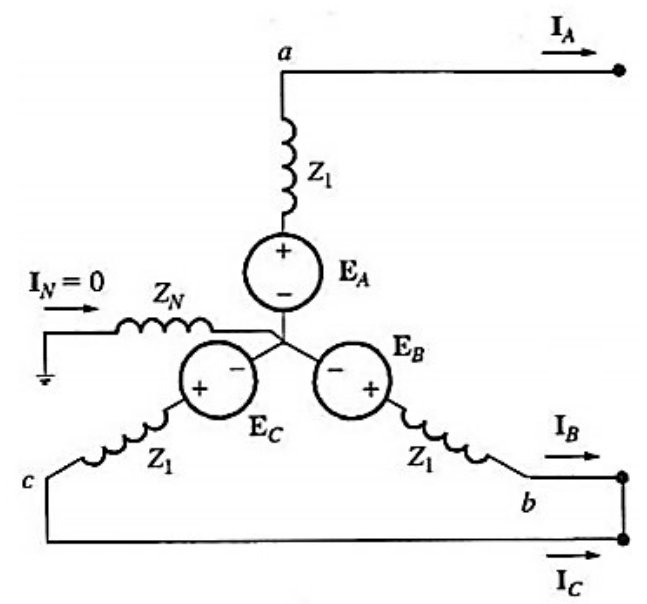

Figure 3.10: A line-to-line fault between phases $b$ and $c$ of an unloaded generator [3]. 


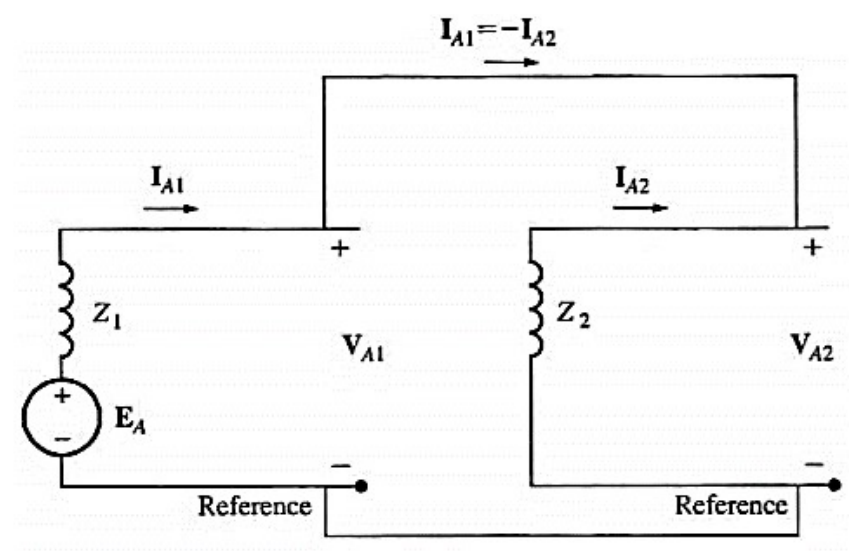

Figure 3.11: For a line-to-line fault, the positive- and negative-sequence networks are connected in parallel to determine the sequence voltages and currents in the fault [3]. 


\subsection{Double Line-to-Ground Fault on an Unloaded Generator}

An unloaded generator with a double line-to-ground fault between phases $b$ and $c$ is shown on page 48 in Figure 3.12. When a double line-to-ground fault occurs between phases $b, c$, and the ground of an unloaded generator, the voltages in phases $b$ and $c$ must both be equal to zero, and the current in phase $a$ must be zero (because that phase is not shorted).

$$
\left.\begin{array}{l}
\mathbf{V}_{B}=0 \\
\mathbf{V}_{C}=0 \\
\mathbf{I}_{A}=0
\end{array}\right\} \text { double line-to-ground fault }
$$

Substituting $\mathbf{V}_{B}=\mathbf{V}_{C}=0$ into Equations (3.16) to (3.18) yields

$$
\begin{aligned}
& \mathbf{V}_{A 0}=\frac{1}{3}\left(\mathbf{V}_{A}+0+0\right)=\frac{1}{3} \mathbf{V}_{A} \\
& \mathbf{V}_{A 1}=\frac{1}{3}\left[\mathbf{V}_{A}+a(0)+a^{2}(0)\right]=\frac{1}{3} \mathbf{V}_{A} \\
& \mathbf{V}_{A 2}=\frac{1}{3}\left[\mathbf{V}_{A}+a^{2}(0)+a(0)\right]=\frac{1}{3} \mathbf{V}_{A}
\end{aligned}
$$

Therefore, $\mathbf{V}_{A 1}=\mathbf{V}_{A 2}=\mathbf{V}_{A 0}$ for a double line-to-ground fault. Furthermore, the fault currents flowing out through phases $b$ and $c$ must return through the neutral, which leads to

$$
\mathbf{I}_{N}=\mathbf{I}_{B}+\mathbf{I}_{C}
$$

Substituting Equations (3.23), (3.24), and (3.28) into Equation (3.59) yields

$$
\begin{aligned}
3 \mathbf{I}_{A 0} & =\left(a^{2} \mathbf{I}_{A 1}+a \mathbf{I}_{A 2}+\mathbf{I}_{A 0}\right)+\left(a \mathbf{I}_{A 1}+a^{2} \mathbf{I}_{A 2}+\mathbf{I}_{A 0}\right) \\
\mathbf{I}_{A 0} & =\left(a^{2}+a\right) \mathbf{I}_{A 1}+\left(a^{2}+a\right) \mathbf{I}_{A 2} \\
\mathbf{I}_{A 0} & =-\mathbf{I}_{A 1}-\mathbf{I}_{A 2}
\end{aligned}
$$

From Equations (3.30) and (3.31) using the fact that $\mathbf{V}_{A 1}=\mathbf{V}_{A 2}$ for a double line-to-ground fault,

$$
\begin{aligned}
\mathbf{E}_{A 1}-\mathbf{I}_{A 1} Z_{1} & =-\mathbf{I}_{A 2} Z_{2} \\
\mathbf{I}_{A 2} & =\frac{\mathbf{I}_{A 1} Z_{1}-\mathbf{E}_{A 1}}{Z_{2}}
\end{aligned}
$$

From Equations (3.30) and (3.32) using the fact that $\mathbf{V}_{A 1}=\mathbf{V}_{A 0}$ for a double line-to-ground fault, 


$$
\begin{aligned}
\mathbf{E}_{A 1}-\mathbf{I}_{A 1} Z_{1} & =-\mathbf{I}_{A 0} Z_{0} \\
\mathbf{I}_{A 0} & =\frac{\mathbf{I}_{A 1} Z_{1}-\mathbf{E}_{A 1}}{Z_{0}}
\end{aligned}
$$

Substituting Equations (3.61) and (3.62) into Equation (3.60) yields

$$
\begin{aligned}
\frac{\mathbf{I}_{A 1} Z_{1}-\mathbf{E}_{A 1}}{Z_{0}} & =-\mathbf{I}_{A 1}-\frac{\mathbf{I}_{A 1} Z_{1}-\mathbf{E}_{A 1}}{Z_{2}} \\
\mathbf{I}_{A 1} & =\frac{\mathbf{E}_{A 1}}{Z_{1}+\frac{Z_{2} Z_{0}}{Z_{2}+Z_{0}}}
\end{aligned}
$$

For a double line-to-ground fault, $\mathbf{V}_{A 1}=\mathbf{V}_{A 2}=\mathbf{V}_{A 0}$, and the total impedance is the sum of $Z_{1}$ plus the parallel combination of $Z_{2}$ and $Z_{0}$. Note that this result is equivalent to connecting the positive-, negative-, and zero-sequence networks in parallel at the terminals representing the location of the fault, as shown on the next page in Figure 3.13.

In summary, to calculate the voltages and currents in a double line-to-ground fault on a three-phase synchronous generator [3]:

1. Create the positive-, negative-, and zero-sequence networks for the generator.

2. Connect the sequence networks in parallel at the terminals representing the location of the fault.

3. Calculate $\mathbf{I}_{A 1}$ from this network.

4. Calculate $\mathbf{V}_{A 0}, \mathbf{V}_{A 1}$, and $\mathbf{V}_{A 2}$ from Equation (3.30), noting that the three voltages are equal.

5. Calculate $\mathbf{I}_{A 0}$ and $\mathbf{I}_{A 2}$ from Equations (3.31) and (3.32).

6. Calculate any required voltages and currents by combining the symmetrical components calculated above using Equations (3.8) to (3.10) and (3.22) to (3.24). 


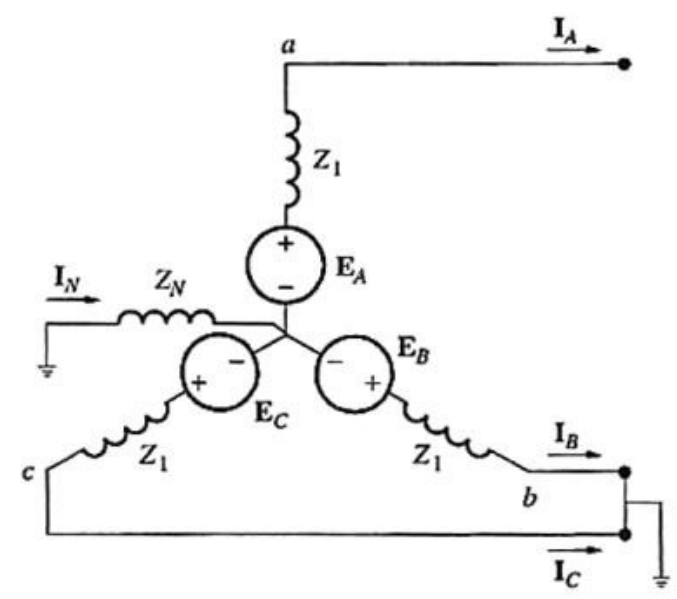

Figure 3.12: A double line-to-ground fault between phases $b, c$, and the ground of an unloaded generator [3].

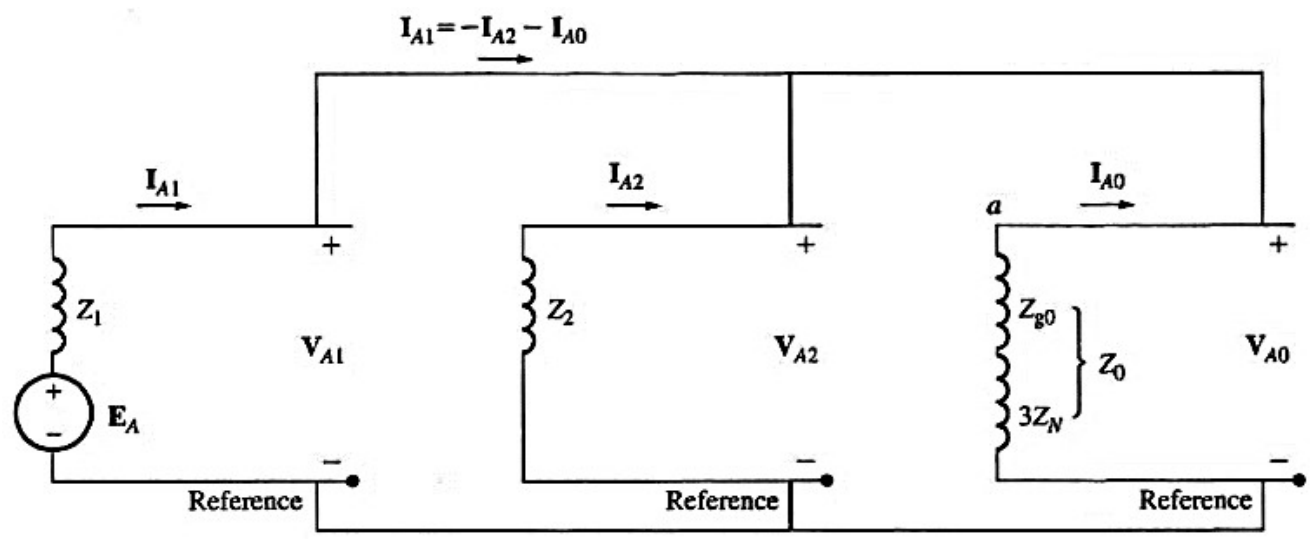

Figure 3.13: For a double line-to-ground fault, the positive-, negative-, and zero-sequence networks are connected in parallel to determine the sequence voltages and currents in the fault [3]. 


\subsection{Unsymmetrical Faults on Power Systems}

The discussion thus far has been limited to calculating the voltages and currents in an unsymmetrical fault on a three-phase synchronous generator. That discussion will now be extended to consider calculating the voltages and currents in an unsymmetrical fault on a more complex power system. To approach the problem of extending the discussion of generators to more complex power systems, consider the application of Thevenin's theorem which states that a linear two-terminal circuit can be replaced by an equivalent circuit consisting of a voltage source in series with an impedance, where the voltage source is the open-circuit voltage at the terminals and the series impedance is the input or equivalent impedance at the terminals when the independent sources are set to zero [1]. To understand the significance of Thevenin's theorem as it pertains to fault analysis, consider the power system shown below in Figure 3.14. Such a power system is sufficiently general for equations derived therefrom to be applicable to any balanced three-phase power system regardless of the complexity. Assume that there is a hypothetical stub of wire connected to each phase of the power system at point " $x$ ", and that a fault occurs on that stub. If a fault occurs on the stub, then a current $I_{f}$ will flow out of the fault. The positive-, negative-, and zero-sequence networks for this power system are shown on the next page in Figure 3.15(a), (c), and (e), respectively, with the location of the fault labelled on each sequence network. Note that each sequence network can be isolated from the rest of the circuit by two terminals, one at the stub and one at the reference. Therefore, a Thevenin equivalent circuit can be created for each sequence network, as seen from the location of the fault. The Thevenin equivalent circuits of the positive-, negative, and zero-sequence networks are shown on the next page to the right of the corresponding sequence network in Figure 3.15(b), (d), and (f), respectively. Note that the Thevenin equivalent circuits of the positive-, negative-, and zero-sequence networks are identical to the positive-, negative-, and zero-sequence equivalent circuits of a generator. Therefore, the discussion thus far regarding unsymmetrical faults on three-phase synchronous generators will equally apply to more complex power systems, if the power systems are represented by their Thevenin equivalent circuits.

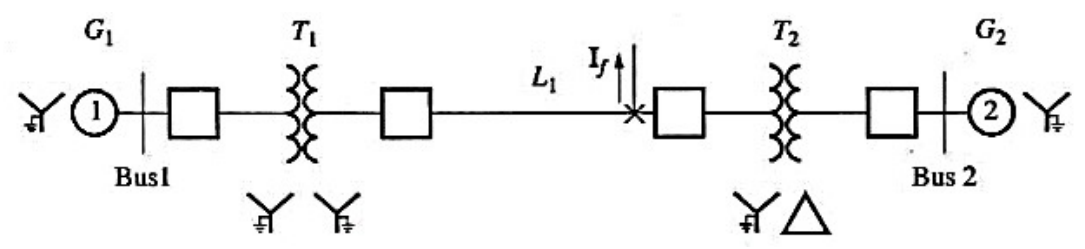

Figure 3.14: A simple power system with a fault at the point indicated by the $\times$ [3]. 


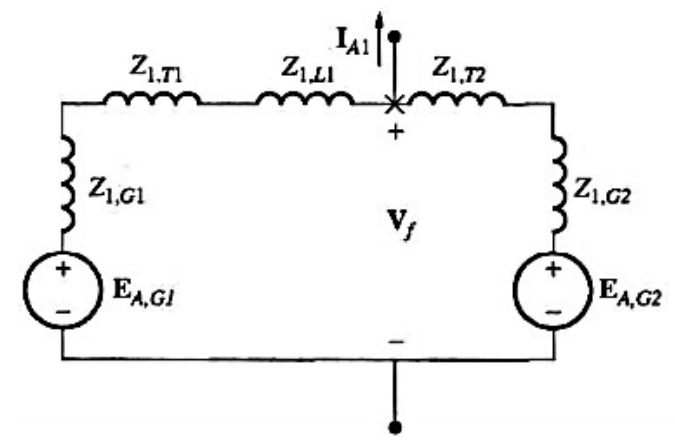

(a)

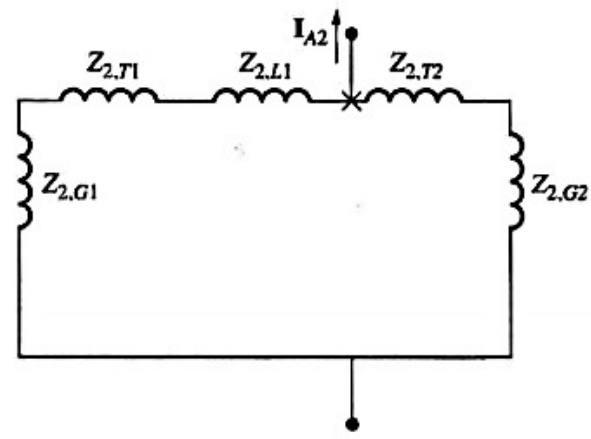

(c)

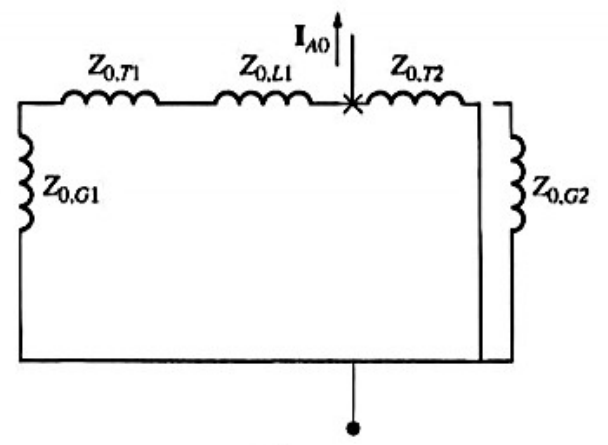

(e)

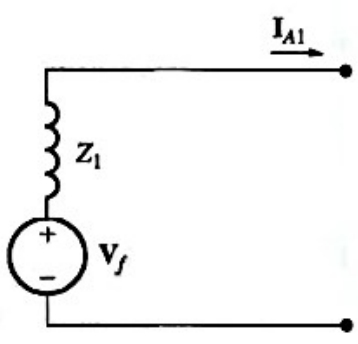

(b)

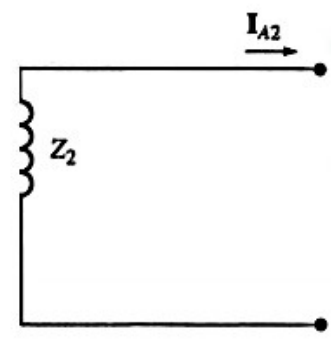

(d)

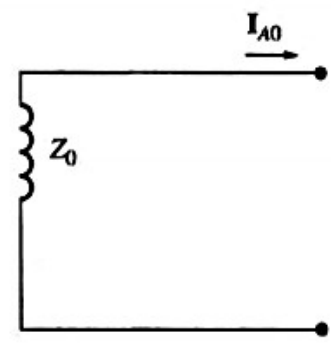

(f)

Figure 3.15: The positive-, negative-, and zero-sequence networks for the power system shown in Figure 3.14, together with their Thevenin equivalent circuits. Note that $\mathbf{V}_{f}$ is the prefault voltage at the location of the fault [3].

For a single line-to-ground fault, the stubs of wire are connected as shown on the next page in Figure 3.16. The following relationships exist at the fault

$$
\left.\begin{array}{rl}
\mathbf{I}_{B} & =0 \\
\mathbf{I}_{C} & =0 \\
\mathbf{V}_{A} & =0
\end{array}\right\} \text { single line-to-ground fault }
$$


These relationships are identical to those for a single line-to-ground fault on a three-phase synchronous generator, thus the symmetrical components of the fault voltages and currents will have the same solutions as for the three-phase synchronous generator, except that the prefault voltage $\mathbf{V}_{f}$ replaces $\mathbf{E}_{A}$. Therefore, the positive-sequence current $\mathbf{I}_{A 1}$ in the fault will be given by the equation

$$
\mathbf{I}_{A 1}=\frac{\mathbf{V}_{f}}{Z_{0}+Z_{1}+Z_{2}}
$$

where $\mathbf{V}_{f}$ is the prefault voltage at the location of the fault and $Z_{1}, Z_{2}$, and $Z_{0}$ are the Thevenin equivalent impedances of the positive-, negative-, and zero-sequence networks, respectively, as seen from the location of the fault. The three sequence networks should be connected in series at the fault point $P$ to calculate the effects of a single line-to-ground fault on the power system, as shown on the next page in Figure 3.17.

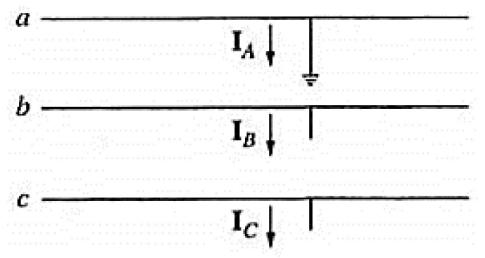

Figure 3.16: Connection diagram for a single line-to-ground fault on a power system [3]. 


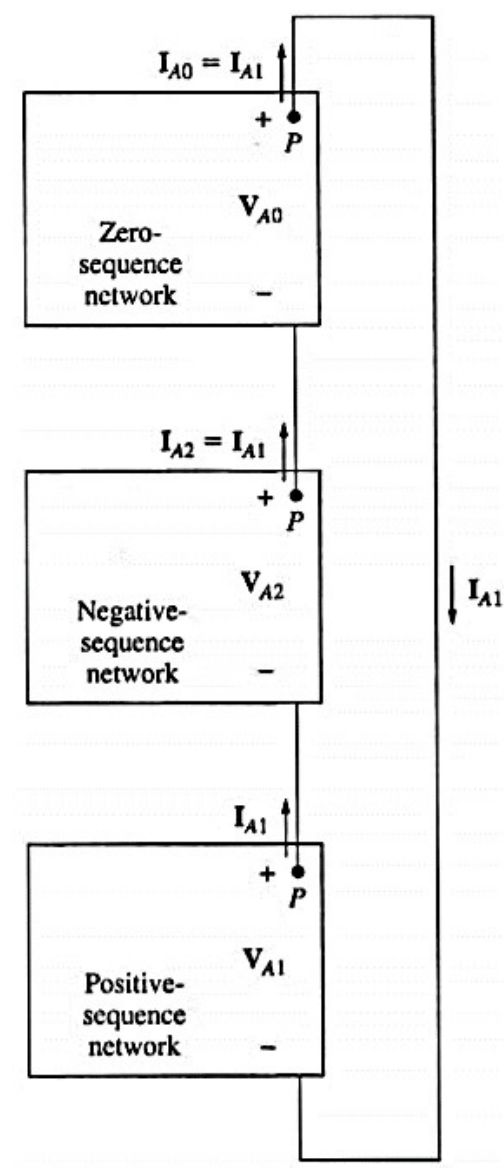

Figure 3.17: For a single line-to-ground fault on a power system, the positive-, negative-, and zero-sequence networks are connected in series at the point of the fault $P$ to determine the sequence voltages and currents in the fault [3].

For a line-to-line fault, the stubs of wire are connected as shown on the next page in Figure 3.18. The following relationships exist at the fault

$$
\left.\begin{array}{rl}
\mathbf{V}_{B} & =\mathbf{V}_{C} \\
\mathbf{I}_{A} & =0 \\
\mathbf{I}_{B} & =-\mathbf{I}_{C}
\end{array}\right\} \text { line-to-line fault }
$$

These relationships are identical to those for a line-to-line fault on a three-phase synchronous generator, thus the symmetrical components of the fault voltages and currents will have the same solutions as for the three-phase synchronous generator, except that the prefault voltage $\mathbf{V}_{f}$ replaces $\mathbf{E}_{A}$. Therefore, the positive-sequence current $\mathbf{I}_{A 1}$ in the fault will be given by the equation

$$
\mathbf{I}_{A 1}=\frac{\mathbf{V}_{f}}{Z_{1}+Z_{2}}
$$


where $\mathbf{V}_{f}$ is the prefault voltage at the location of the fault and $Z_{1}$ and $Z_{2}$ are the Thevenin equivalent impedances of the positive- and negative-sequence networks, respectively, as seen from the location of the fault. The positive- and negative-sequence networks should be connected in parallel at the fault point $P$ to calculate the effects of a line-to-line fault on the power system, as shown below in Figure 3.19.

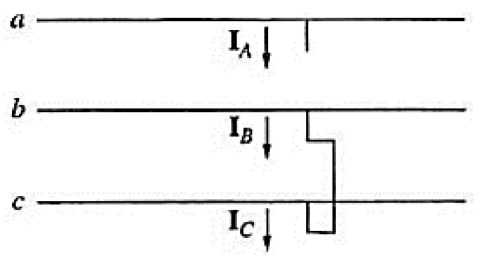

Figure 3.18: Connection diagram for a line-to-line fault on a power system [3].

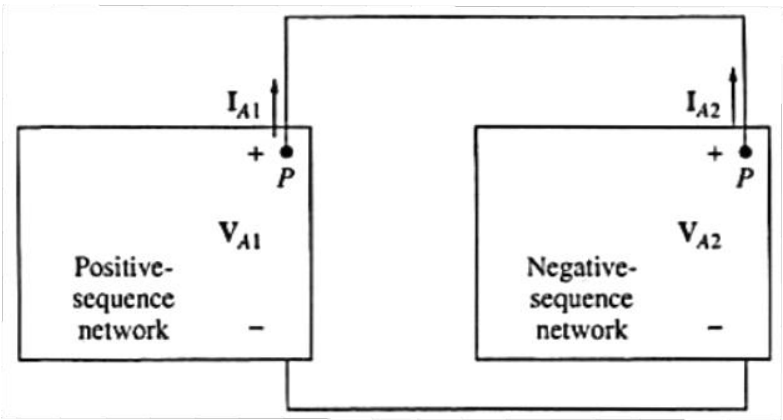

Figure 3.19: For a line-to-line fault on a power system, the positive- and negative-sequence networks are connected in parallel at the point of the fault $P$ to determine the sequence voltages and currents in the fault [3].

For a double line-to-ground fault, the stubs of wire are connected as shown on the next page in Figure 3.20. The following relationships exist at the fault

$$
\left.\begin{array}{l}
\mathbf{V}_{B}=0 \\
\mathbf{V}_{C}=0 \\
\mathbf{I}_{A}=0
\end{array}\right\} \text { double line-to-ground fault }
$$

These relationships are identical to those for a double line-to-ground fault on a three-phase synchronous generator, thus the symmetrical components of the fault voltages and currents will have the same solutions as for the three-phase synchronous generator, except that the prefault voltage $\mathbf{V}_{f}$ replaces $\mathbf{E}_{A}$. Therefore, the positive-sequence current $\mathbf{I}_{A 1}$ in the fault will be given by the equation 


$$
\mathbf{I}_{A 1}=\frac{\mathbf{v}_{f}}{Z_{1}+\frac{Z_{2} Z_{0}}{Z_{2}+Z_{0}}}
$$

where $\mathbf{V}_{f}$ is the prefault voltage at the location of the fault and $Z_{1}, Z_{2}$, and $Z_{0}$ are the Thevenin equivalent impedances of the positive-, negative-, and zero-sequence networks, respectively, as seen from the location of the fault. The three sequence networks should be connected in parallel at the fault point $P$ to calculate the effects of a double line-to-ground fault on the power system, as shown below in Figure 3.21.

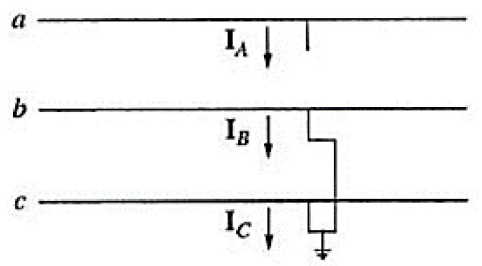

Figure 3.20: Connection diagram for a double line-to-ground fault on a power system [3].

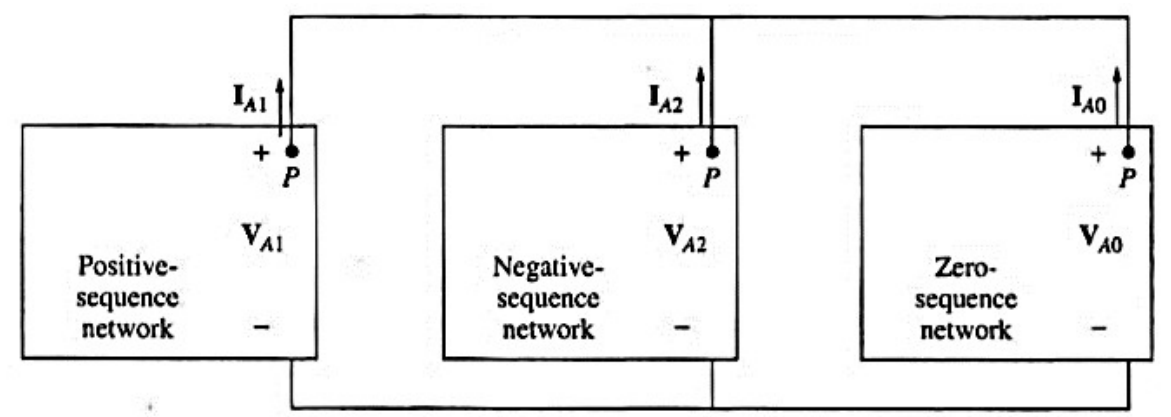

Figure 3.21: For a double line-to-ground fault on a power system, the positive-, negative-, and zero-sequence networks are connected in parallel at the point of the fault $P$ to determine the sequence voltages and currents in the fault [3]. 


\subsection{Unsymmetrical Fault Analysis using the Bus Impedance Matrix}

In the previous section, the positive-sequence bus impedance matrix $\mathbf{Z}_{\text {bus }}$ was used to calculate the fault current in a symmetrical three-phase fault, as well as the voltages and currents at each bus and in any desired transmission lines in the power system during the fault. It is also possible to create bus impedance matrices for negative- and zero-sequence networks, which can be used to calculate the voltages and currents for unsymmetrical faults in a power system. If a sequence network is represented by a bus impedance matrix $\mathbf{Z}_{\text {bus }}$, then the on-diagonal terms of the bus impedance matrix $Z_{i i}$ are simply the Thevenin equivalent impedances of the network at the corresponding buses. Therefore, if a single line-toground fault occurs at bus $i$, then the positive-sequence fault current at bus $i$ can be calculated from the equation

$$
\mathbf{I}_{A 1}=\frac{\mathbf{V}_{f}}{Z_{i i, 1}+Z_{i i, 2}+Z_{i i, 0}}
$$

where $Z_{i i, 1}$ is the impedance of element $i i$ of the positive-sequence bus impedance matrix, $Z_{i i, 2}$ is the impedance of element $i i$ of the negative-sequence bus impedance matrix, and $Z_{i i, 0}$ is the impedance of element $i i$ of the zero-sequence bus impedance matrix.

Similarly, if a line-to-line fault occurs at bus $i$, then the positive-sequence fault current at bus $i$ can be calculated from the equation

$$
\mathbf{I}_{A 1}=\frac{\mathbf{V}_{f}}{Z_{i i, 1}+Z_{i i, 2}}
$$

Finally, if a double line-to-ground fault occurs at bus $i$, then the positive-sequence fault current at bus $i$ can be calculated from the equation

$$
\mathbf{I}_{A 1}=\frac{\mathbf{v}_{f}}{Z_{i i, 1}+\frac{Z_{i i, 2} Z_{i i, 0}}{Z_{i i, 2}+Z_{i i, 0}}}
$$




\subsection{Faults Through an Impedance}

The discussion thus far has been limited to faults with zero fault impedance, i.e., a solid or "bolted" fault. Such faults produce the highest value of fault currents for a given type of fault and form the basis of calculations used to determine the maximum possible fault currents that a power system must be able to withstand without being destroyed. Although such faults result in the highest value of fault currents and are therefore the most conservative values to use when determining the effects of anticipated faults on a power system, the fault impedance is seldom zero [1]. Most faults are the result of insulator flashover after a lightning strike, where the impedance between the transmission line and the ground depends on the resistance of the arc, of the tower structure itself, and of the ground connection if there are no overhead ground wires (e.g., the tower footing). These impedances reduce the total current flow compared to the solid or bolted case.

A symmetrical three-phase fault through an impedance $Z_{f}$ is shown on page 60 in Figure 3.22(a). Note that the three-phase power system remains balanced during the fault having the same impedance between each phase and the common point. Since the power system remains balanced, only positive-sequence components of voltage and current appear in the fault. With the fault impedance $Z_{f}$ equal in all phases, the impedance $Z_{f}$ is simply connected in series with the positive-sequence network at the fault point $P$ to calculate the effects of a symmetrical three-phase fault through an impedance, as shown on page 62 in Figure 3.23(a). Therefore, the positive-sequence current in the fault becomes

$$
\mathbf{I}_{A 1}=\frac{\mathbf{V}_{f}}{Z_{1}+Z_{f}}
$$

A single line-to-ground fault through an impedance $Z_{f}$ is shown on page 60 in Figure 3.22(b). The following relationships exist at the fault

$$
\left.\begin{array}{rl}
\mathbf{I}_{B} & =0 \\
\mathbf{I}_{C} & =0 \\
\mathbf{V}_{A} & =\mathbf{I}_{A} Z_{f}
\end{array}\right\} \text { single line-to-ground fault }
$$

The symmetrical components of current in the fault are given in terms of the currents in each phase by Equations (3.25) to (3.27). Substituting $\mathbf{I}_{B}=0$ and $\mathbf{I}_{C}=0$ into Equations (3.25) to (3.27) yields 


$$
\begin{aligned}
& \mathbf{I}_{A 0}=\frac{1}{3}\left(\mathbf{I}_{A}+0+0\right)=\frac{1}{3} \mathbf{I}_{A} \\
& \mathbf{I}_{A 1}=\frac{1}{3}\left[\mathbf{I}_{A}+a(0)+a^{2}(0)\right]=\frac{1}{3} \mathbf{I}_{A} \\
& \mathbf{I}_{A 2}=\frac{1}{3}\left[\mathbf{I}_{A}+a^{2}(0)+a(0)\right]=\frac{1}{3} \mathbf{I}_{A}
\end{aligned}
$$

Therefore,

$$
\mathbf{I}_{A 0}=\mathbf{I}_{A 1}=\mathbf{I}_{A 2}=\frac{1}{3} \mathbf{I}_{A}
$$

Equation (3.78) shows that the symmetrical components of current are all equal in a single line-to-ground fault through an impedance $Z_{f}$. Substituting $\mathbf{I}_{A 2}=\mathbf{I}_{A 1}$ and $\mathbf{I}_{A 0}=\mathbf{I}_{A 1}$ into Equations (3.30) to (3.32) and replacing $\mathbf{E}_{A 1}$ with the prefault voltage $\mathbf{V}_{f}$ yields

$$
\begin{aligned}
& \mathbf{V}_{A 1}=\mathbf{V}_{f}-\mathbf{I}_{A 1} Z_{1} \\
& \mathbf{V}_{A 2}=-\mathbf{I}_{A 1} Z_{2} \\
& \mathbf{V}_{A 0}=-\mathbf{I}_{A 1} Z_{0}
\end{aligned}
$$

The voltage in phase $a$ is given in terms of symmetrical components by Equation (3.8). Substituting Equations (3.79) to (3.81) into Equation (3.8) yields

$$
\mathbf{V}_{A}=\mathbf{V}_{A 1}+\mathbf{V}_{A 2}+\mathbf{V}_{A 0}=\mathbf{V}_{f}-\mathbf{I}_{A 1} Z_{1}-\mathbf{I}_{A 1} Z_{2}-\mathbf{I}_{A 1} Z_{0}=3 \mathbf{I}_{A 1} Z_{f}
$$

Rearranging Equation (3.82) to solve for the positive-sequence current in the fault yields

$$
\mathbf{I}_{A 1}=\frac{\mathbf{v}_{f}}{Z_{1}+Z_{2}+Z_{0}+3 Z_{f}}
$$

For a single line-to-ground fault through an impedance, the positive-, negative-, and zero-sequence current are all equal, and the total impedance is the series combination of $Z_{1}, Z_{2}, Z_{0}$, and $3 Z_{f}$. This makes sense physically because the impedance $Z_{f}$ appears in the path to ground, hence the zero-sequence components of current can flow through it, and the zero-sequence components of current from all three phases will flow through the same impedance $Z_{f}$. Therefore, the fault impedance must be connected in series with the zerosequence network at the fault point $P$ in order for the zero-sequence current to flow through it, as shown on page 62 in Figure 3.23(b). The size of the fault impedance in the sequence diagram of Figure 3.23(b) must be $3 Z_{f}$ to account for the fact that the zero-sequence currents from all three phases flow through it and the current is identical in each phase. 
A line-to-line fault through an impedance $Z_{f}$ is shown on page 60 in Figure 3.22(c). The following relationships exist at the fault

$$
\left.\begin{array}{rl}
\mathbf{V}_{C} & =\mathbf{V}_{B}-\mathbf{I}_{B} Z_{f} \\
\mathbf{I}_{A} & =0 \\
\mathbf{I}_{B} & =-\mathbf{I}_{C}
\end{array}\right\} \text { line-to-line fault }
$$

Substituting $\mathbf{V}_{C}=\mathbf{V}_{B}-\mathbf{I}_{B} Z_{f}$ into Equations (3.16) to (3.18) yields

$$
\begin{aligned}
& \mathbf{V}_{A 0}=\frac{1}{3}\left[\mathbf{V}_{A}+\mathbf{V}_{B}+\left(\mathbf{V}_{B}-\mathbf{I}_{B} Z_{f}\right)\right] \\
& \mathbf{V}_{A 1}=\frac{1}{3}\left[\mathbf{V}_{A}+a \mathbf{V}_{B}+a^{2}\left(\mathbf{V}_{B}-\mathbf{I}_{B} Z_{f}\right)\right] \\
& \mathbf{V}_{A 2}=\frac{1}{3}\left[\mathbf{V}_{A}+a^{2} \mathbf{V}_{B}+a\left(\mathbf{V}_{B}-\mathbf{I}_{B} Z_{f}\right)\right]
\end{aligned}
$$

From Equations (3.86) and (3.87),

$$
\mathbf{V}_{A 2}=\mathbf{V}_{A 1}+\frac{1}{3}\left(a^{2}-a\right) \mathbf{I}_{B} Z_{f}
$$

Substituting Equation (3.23) into Equation (3.88) yields

$$
\mathbf{V}_{A 2}=\mathbf{V}_{A 1}+\frac{1}{3}\left(a^{2}-a\right)\left(a^{2} \mathbf{I}_{A 1}+a \mathbf{I}_{A 2}+\mathbf{I}_{A 0}\right) Z_{f}
$$

The symmetrical components of current in the fault are given in terms of the currents in each phase by Equations (3.25) to (3.27). Substituting $\mathbf{I}_{A}=0$ and $\mathbf{I}_{B}=-\mathbf{I}_{C}$ into Equations (3.25) to (3.27) yields

$$
\begin{aligned}
& \mathbf{I}_{A 0}=\frac{1}{3}\left(0-\mathbf{I}_{C}+\mathbf{I}_{C}\right) \\
& \mathbf{I}_{A 1}=\frac{1}{3}\left[0-a \mathbf{I}_{C}+a^{2} \mathbf{I}_{C}\right] \\
& \mathbf{I}_{A 2}=\frac{1}{3}\left[0-a^{2} \mathbf{I}_{C}+a \mathbf{I}_{C}\right]
\end{aligned}
$$

From Equations (3.90) to (3.92), it can readily be seen that $\mathbf{I}_{A 0}=0$ and $\mathbf{I}_{A 2}=-\mathbf{I}_{A 1}$. Substituting $\mathbf{I}_{A 0}=0$ and $\mathbf{I}_{A 2}=-\mathbf{I}_{A 1}$ into Equation (3.89) yields 


$$
\begin{aligned}
\mathbf{V}_{A 2} & =\mathbf{V}_{A 1}+\frac{1}{3}\left(a^{2}-a\right)\left(a^{2} \mathbf{I}_{A 1}-a \mathbf{I}_{A 1}\right) Z_{f} \\
& =\mathbf{V}_{A 1}+\frac{1}{3}\left(a^{2}-a\right)^{2} \mathbf{I}_{A 1} Z_{f} \\
& =\mathbf{V}_{A 1}+\frac{1}{3}(-j \sqrt{3})^{2} \mathbf{I}_{A 1} Z_{f} \\
& =\mathbf{V}_{A 1}-\mathbf{I}_{A 1} Z_{f}
\end{aligned}
$$

Substituting $\mathbf{V}_{A 2}=\mathbf{V}_{A 1}-\mathbf{I}_{A 1} Z_{f}, \mathbf{I}_{A 0}=0$, and $\mathbf{I}_{A 2}=-\mathbf{I}_{A 1}$ into Equations (3.30) to (3.32) and replacing $\mathbf{E}_{A 1}$ with the prefault voltage $\mathbf{V}_{f}$ yields

$$
\begin{aligned}
& \mathbf{V}_{A 1}=\mathbf{V}_{f}-\mathbf{I}_{A 1} Z_{1} \\
& \mathbf{V}_{A 1}=\mathbf{I}_{A 1} Z_{2}+\mathbf{I}_{A 1} Z_{f} \\
& \mathbf{V}_{A 0}=-\mathbf{I}_{A 0} Z_{0}=0
\end{aligned}
$$

From Equations (3.94) and (3.95), it can be observed that

$$
\begin{gathered}
\mathbf{I}_{A 1} Z_{2}+\mathbf{I}_{A 1} Z_{f}=\mathbf{V}_{f}-\mathbf{I}_{A 1} Z_{1} \\
\mathbf{I}_{A 1}=\frac{\mathbf{V}_{f}}{Z_{1}+Z_{2}+Z_{f}}
\end{gathered}
$$

For a line-to-line fault through an impedance, the positive-sequence current is the negative of the negativesequence current, and the zero-sequence current is zero. In addition, the positive- and negative-sequence voltages differ by only the voltage drop across the impedance $Z_{f}$ (i.e., $\mathbf{V}_{A 1}-\mathbf{V}_{A 2}=\mathbf{I}_{A 1} Z_{f}$ ). Therefore, the impedance $Z_{f}$ must be inserted between the positive- and negative-sequence networks at the fault point $P$ such that the corresponding voltages differ by only the voltage drop across the impedance $Z_{f}$, as shown on page 62 in Figure 3.23(c). 


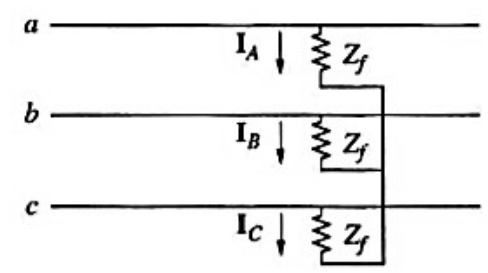

(a)

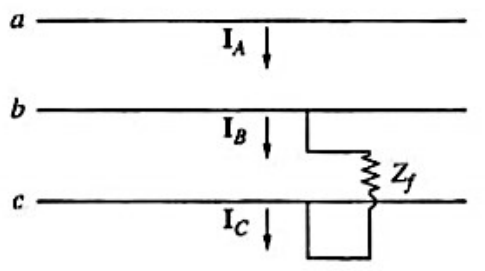

(c)

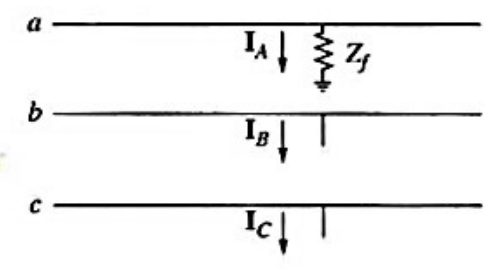

(b)

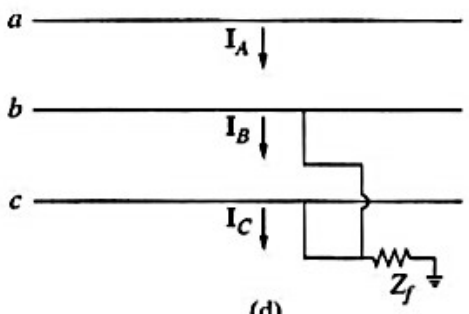

Figure 3.22: Connection diagrams for various faults through an impedance on a power system: (a) Symmetrical threephase fault; (b) Single line-to-ground fault; (c) Line-to-line fault; (d) Double line-to-ground fault [3].

A double line-to-ground fault through an impedance $Z_{f}$ is shown above in Figure 3.22(d). The following relationships exist at the fault

$$
\left.\begin{array}{l}
\mathbf{V}_{B}=\left(\mathbf{I}_{B}+\mathbf{I}_{C}\right) Z_{f} \\
\mathbf{V}_{C}=\mathbf{V}_{B} \\
\mathbf{I}_{A}=0
\end{array}\right\} \text { double line-to-ground fault }
$$

Substituting $\mathbf{V}_{C}=\mathbf{V}_{B}$ into Equations (3.16) to (3.18) yields

$$
\begin{aligned}
& \mathbf{V}_{A 0}=\frac{1}{3}\left(\mathbf{V}_{A}+\mathbf{V}_{B}+\mathbf{V}_{B}\right)=\frac{1}{3}\left(\mathbf{V}_{A}+2 \mathbf{V}_{B}\right) \\
& \mathbf{V}_{A 1}=\frac{1}{3}\left(\mathbf{V}_{A}+a \mathbf{V}_{B}+a^{2} \mathbf{V}_{B}\right)=\frac{1}{3}\left(\mathbf{V}_{A}-\mathbf{V}_{B}\right) \\
& \mathbf{V}_{A 2}=\frac{1}{3}\left(\mathbf{V}_{A}+a^{2} \mathbf{V}_{B}+a \mathbf{V}_{B}\right)=\frac{1}{3}\left(\mathbf{V}_{A}-\mathbf{V}_{B}\right)
\end{aligned}
$$

Therefore, $\mathbf{V}_{A 1}=\mathbf{V}_{A 2}=\mathbf{V}_{A 0}-\mathbf{V}_{B}=\mathbf{V}_{A 0}-\left(\mathbf{I}_{B}+\mathbf{I}_{C}\right) Z_{f}$ for this type of fault. Furthermore, the fault currents flowing out through phases $b$ and $c$ must return through the neutral, which leads to

$$
\mathbf{I}_{N}=\mathbf{I}_{B}+\mathbf{I}_{C}
$$

From Equations (3.28) and (3.103), $\mathbf{V}_{A 1}=\mathbf{V}_{A 2}=\mathbf{V}_{A 0}-\mathbf{I}_{N} Z_{f}=\mathbf{V}_{A 0}-3 \mathbf{I}_{A 0} Z_{f}$ for this type of fault. Substituting Equations (3.23), (3.24), and (3.28) into Equation (3.103) yields 


$$
\begin{aligned}
3 \mathbf{I}_{A 0} & =\left(a^{2} \mathbf{I}_{A 1}+a \mathbf{I}_{A 2}+\mathbf{I}_{A 0}\right)+\left(a \mathbf{I}_{A 1}+a^{2} \mathbf{I}_{A 2}+\mathbf{I}_{A 0}\right) \\
\mathbf{I}_{A 0} & =\left(a^{2}+a\right) \mathbf{I}_{A 1}+\left(a^{2}+a\right) \mathbf{I}_{A 2} \\
\mathbf{I}_{A 0} & =-\mathbf{I}_{A 1}-\mathbf{I}_{A 2}
\end{aligned}
$$

From Equations (3.30) and (3.31) using the fact that $\mathbf{V}_{A 1}=\mathbf{V}_{A 2}$ for this type of fault and replacing $\mathbf{E}_{A 1}$ with the prefault voltage $\mathbf{V}_{f}$,

$$
\begin{aligned}
\mathbf{V}_{f}-\mathbf{I}_{A 1} Z_{1} & =-\mathbf{I}_{A 2} Z_{2} \\
\mathbf{I}_{A 2} & =\frac{\mathbf{I}_{A 1} Z_{1}-\mathbf{V}_{f}}{Z_{2}}
\end{aligned}
$$

From Equations (3.30) and (3.32) using the fact that $\mathbf{V}_{A 1}=\mathbf{V}_{A 0}-3 \mathbf{I}_{A 0} Z_{f}$ for this type of fault and replacing $\mathbf{E}_{A 1}$ with the prefault voltage $\mathbf{V}_{f}$,

$$
\begin{aligned}
\mathbf{V}_{f}-\mathbf{I}_{A 1} Z_{1} & =-\mathbf{I}_{A 0} Z_{0}-3 \mathbf{I}_{A 0} Z_{f} \\
\mathbf{I}_{A 0} & =\frac{\mathbf{I}_{A 1} Z_{1}-\mathbf{V}_{f}}{Z_{0}+3 Z_{f}}
\end{aligned}
$$

Substituting Equations (3.105) and (3.106) into Equation (3.104) yields

$$
\begin{array}{r}
\frac{\mathbf{I}_{A 1} Z_{1}-\mathbf{v}_{f}}{Z_{0}+3 Z_{f}}=-\mathbf{I}_{A 1}-\frac{\mathbf{I}_{A 1} Z_{1}-\mathbf{v}_{f}}{Z_{2}} \\
\mathbf{I}_{A 1}=\frac{\mathbf{v}_{f}}{Z_{1}+\frac{Z_{2}\left(Z_{0}+3 Z_{f}\right)}{Z_{2}+Z_{0}+3 Z_{f}}}
\end{array}
$$

For a double line-to-ground fault through an impedance, $\mathbf{V}_{A 1}=\mathbf{V}_{A 2}=\mathbf{V}_{A 0}-3 \mathbf{I}_{A 0} Z_{f}$, and the total impedance is the sum of $Z_{1}$ plus the parallel combination of $Z_{2}$ and $Z_{0}+3 Z_{f}$. This makes sense physically because the impedance $Z_{f}$ appears in the path to ground, hence the zero-sequence components of current can flow through it, and the zero-sequence components of current from all three phases will flow through the same impedance $Z_{f}$. Therefore, the fault impedance must be connected in series with the zerosequence network at the fault point $P$ in order for the zero-sequence current to flow through it, as shown on the next page in Figure 3.23(d). The size of the fault impedance in the sequence diagram of Figure 3.23(d) must be $3 Z_{f}$ to account for the fact that the zero-sequence currents from all three phases flow through it and the current is identical in each phase. 


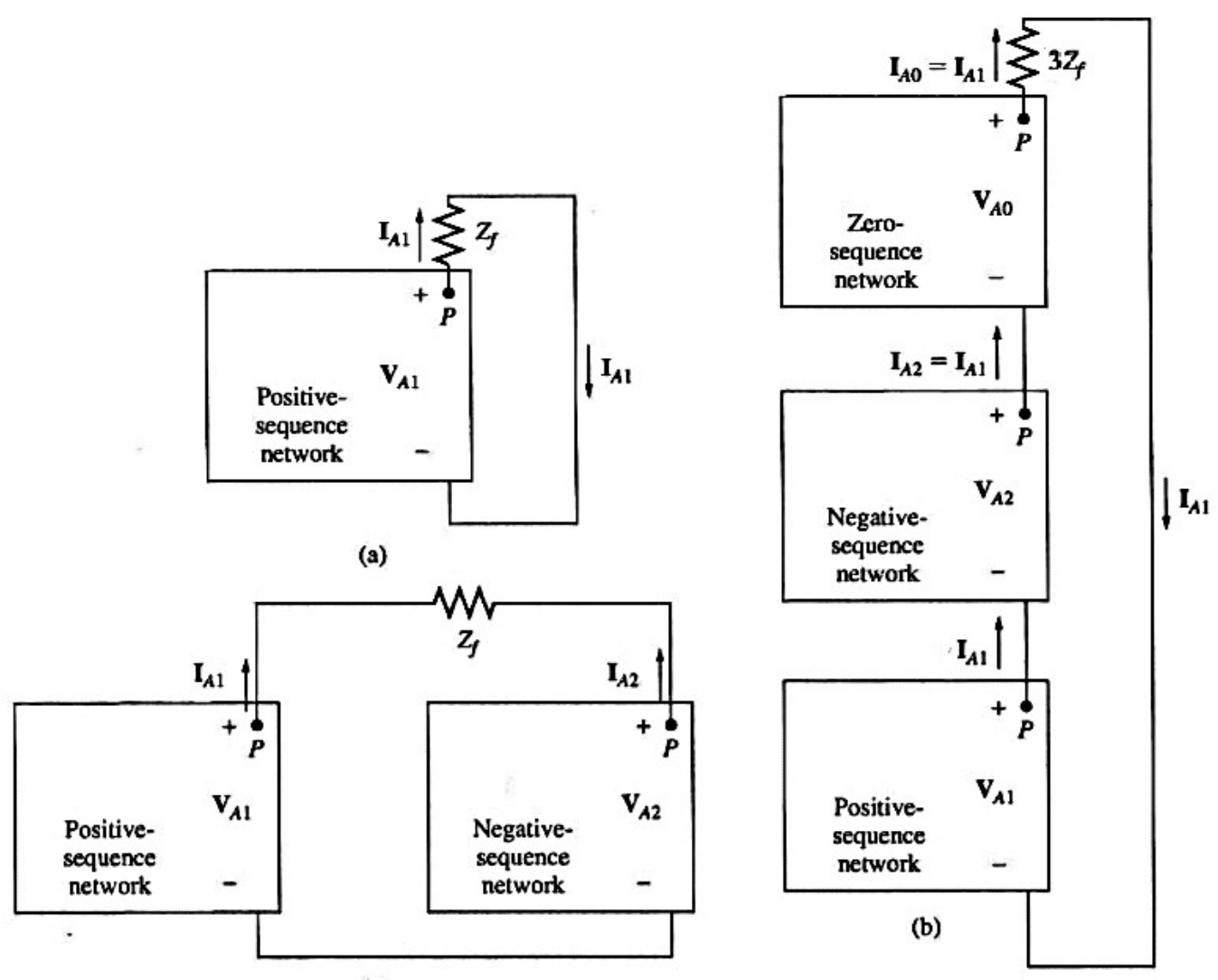

(c)

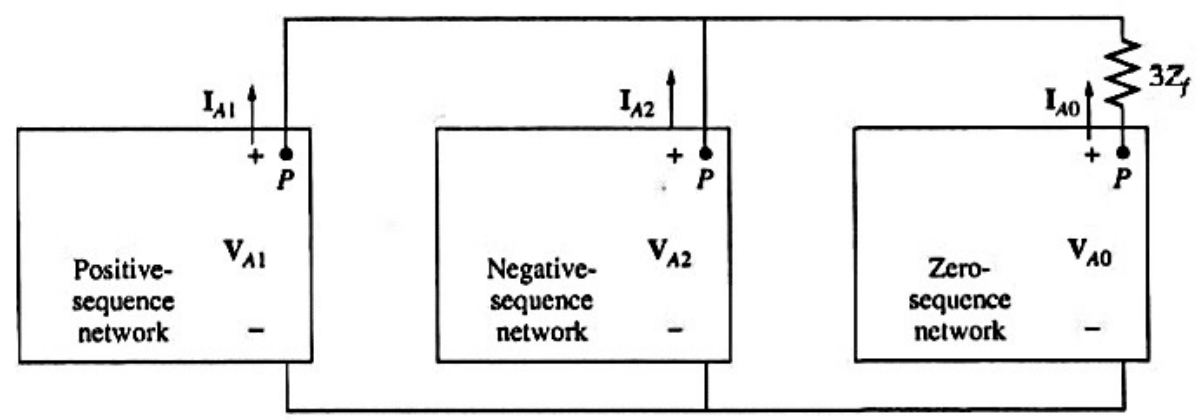

(d)

Figure 3.23: Connections of sequence networks to simulate faults through an impedance on a power system: (a) Symmetrical three-phase fault; (b) Single line-to-ground fault; (c) Line-to-line fault; (d) Double line-to-ground fault [3]. 


\subsection{Chapter Summary}

Unbalanced three-phase voltages and currents can be resolved into their symmetrical components. There are three sets of symmetrical components: a positive-sequence $(a b c)$ set, a negative-sequence $(a c b)$ set, and a zero-sequence (in phase) set. Positive-sequence components peak in phase order $a b c$, while negative-sequence components peak in phase order $a c b$, and zero-sequence components all peak at the same time. Problems are solved with symmetrical components by treating each set of symmetrical components separately and superimposing the results.

Currents of a given sequence produce voltages of that sequence only, so it is possible to create separate positive-, negative-, and zero-sequence per-unit equivalent circuits (called sequence networks), and to solve for the voltage and current relationships in each one separately.

The positive-sequence network alone is sufficient to solve problems involving symmetrical three-phase faults, because those power systems are balanced. If the power system becomes unbalanced for some reason (such as a single line-to-ground fault), then all the phase sequences are needed.

The fault currents in a single line-to-ground fault can be calculated by connecting the three sequence networks in series so that $\mathbf{I}_{A 1}=\mathbf{I}_{A 2}=\mathbf{I}_{A 0}$. Then, the positive-sequence current $\mathbf{I}_{A 1}$ in the fault becomes

$$
\mathbf{I}_{A 1}=\frac{\mathbf{V}_{f}}{Z_{0}+Z_{1}+Z_{2}}
$$

where $\mathbf{V}_{f}$ is the prefault voltage on the bus where the fault occurs, and $Z_{1}, Z_{2}$, and $Z_{0}$ are the Thevenin equivalent impedances of the positive-, negative-, and zero-sequence networks, respectively, at the point of the fault.

The fault currents in a line-to-line fault can be calculated by connecting the positive- and negative-sequence networks in parallel so that $\mathbf{V}_{A 1}=\mathbf{V}_{A 2}$ and $\mathbf{I}_{A 1}=-\mathbf{I}_{A 2}$. Then, the positive-sequence current $\mathbf{I}_{A 1}$ in the fault becomes

$$
\mathbf{I}_{A 1}=\frac{\mathbf{V}_{f}}{Z_{1}+Z_{2}}
$$


where $\mathbf{V}_{f}$ is the prefault voltage on the bus where the fault occurs, and $Z_{1}$ and $Z_{2}$ are the Thevenin equivalent impedances of the positive- and negative-sequence networks, respectively, at the point of the fault.

The fault currents in a double line-to-ground fault can be calculated by connecting the positive-, negative-, and zero-sequence networks in parallel so that $\mathbf{V}_{A 1}=\mathbf{V}_{A 2}=\mathbf{V}_{A 0}$ and $\mathbf{I}_{A 1}=-\mathbf{I}_{A 2}-\mathbf{I}_{A 0}$. Then, the positivesequence current $\mathbf{I}_{A 1}$ in the fault becomes

$$
\mathbf{I}_{A 1}=\frac{\mathbf{V}_{f}}{Z_{1}+\frac{Z_{2} Z_{0}}{Z_{2}+Z_{0}}}
$$

where $\mathbf{V}_{f}$ is the prefault voltage on the bus where the fault occurs, and $Z_{1}, Z_{2}$, and $Z_{0}$ are the Thevenin equivalent impedances of the positive-, negative-, and zero-sequence networks, respectively, at the point of the fault.

In any type of fault, once the positive-sequence current $\mathbf{I}_{A 1}$ in the fault has been calculated, it is relatively straightforward to calculate the negative- and zero-sequence currents $\mathbf{I}_{A 2}$ and $\mathbf{I}_{A 0}$, respectively, from the special relationships that apply to the particular type of fault. Then the actual faults currents in each phase can be calculated from the equations

$$
\begin{aligned}
& \mathbf{I}_{A}=\mathbf{I}_{A 1}+\mathbf{I}_{A 2}+\mathbf{I}_{A 0} \\
& \mathbf{I}_{B}=a^{2} \mathbf{I}_{A 1}+a \mathbf{I}_{A 2}+\mathbf{I}_{A 0} \\
& \mathbf{I}_{C}=a \mathbf{I}_{A 1}+a^{2} \mathbf{I}_{A 2}+\mathbf{I}_{A 0}
\end{aligned}
$$

It is possible to create separate positive-, negative-, and zero-sequence bus admittance matrices $\mathbf{Y}_{\text {bus }}$ and bus impedance matrices $\mathbf{Z}_{\text {bus }}$ for a power system. Since the on-diagonal terms of the bus impedance matrix $Z_{i i}$ contains the Thevenin equivalent impedance of the power system at that point, these terms can be used in the equations for the positive-sequence current $\mathbf{I}_{A 1}$ to calculate the fault currents that would flow at each bus in the power system for each type of fault. 


\section{Chapter 4 - Power System Modeling and Fault Analysis of Converter-Based Distributed Energy Resources}

\subsection{Introduction}

Electric power systems have used and will continue to use synchronous machines in the foreseeable future for the generation of electricity. However, with emerging concerns over climate change and the need for reduced greenhouse gas emissions, together with the growing awareness of the importance of the natural environment and the depletion of the earth's non-renewable energy resources, the generation of electricity from distributed renewable energy resources such as solar photovoltaic (PV) and wind energy has begun to expand at a rapid pace. Generally, such generation systems either generate dc or use asynchronous machines that generate incompatible frequencies, and therefore must be interfaced to the three-phase power system through a power electronics converter. The combination of synchronous and converter isolated electrical generation systems is expected to change the behaviour of three-phase power systems following disturbances such as the occurrence of a fault on the power system. Proliferation of converterbased distributed energy resources in distribution systems has introduced new challenges in determining the maximum possible fault currents that a power system must be able to withstand without being compromised. As part of a connection impact assessment for a new generation facility, utilities require detailed information on fault characteristics of the generation sources. Although IEEE Std 1547-2018 [18] provides requirements relevant to the interconnection and interoperability between power systems and distributed energy resources, there is still limited knowledge or contradictory conclusions regarding the behaviour of converter-based distributed energy resources (e.g., solar PV and wind power generation stations) during faults on transmission or distribution systems. Without accurate data and details of the fault current contributions from series converter-connected solar PV and wind generators, it is not possible to assess the risk of connecting them and hence determining the strategy and settings of the overcurrent protective devices that protect the power system. Therefore, it is imperative to develop the mathematical and software simulation models that approximate the response of converter-based distributed energy resources during a fault on the transmission or distribution system in order to determine the fault current contributions to the electrical grid that a transmission or distribution utility needs to reflect in their connection impact assessments. A single line diagram of a wind turbine generator together with flywheel energy storage interfaced to the three-phase power system through a power electronics converter is shown on the next page in Figure 4.1(a), followed by a single line diagram of a microgrid topology with solar PV and wind power generation options together with battery bank and supercapacitor storage options interfaced to the three-phase power system through a power electronics converter shown on the next page in Figure 4.1(b). 


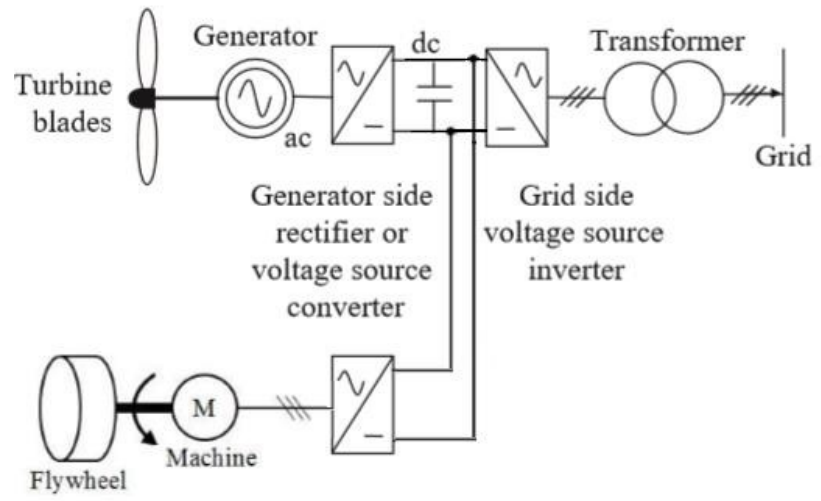

(a)

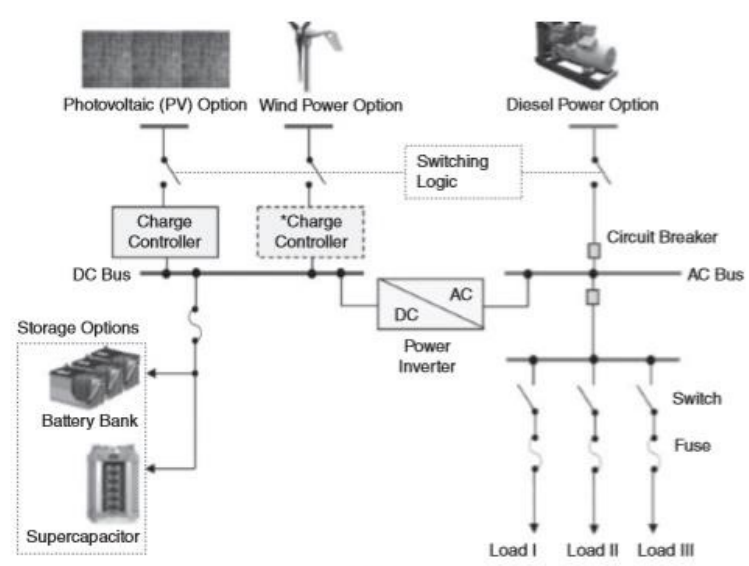

(b)

Figure 4.1: (a) Series converter-connected wind turbine generator together with flywheel energy storage [5]. (b) Microgrid topology with series converter-connected solar PV and wind power generation options together with battery bank and supercapacitor storage options [19]. 


\subsection{Problem Statement}

Although IEEE Std 1547-2018 [18] provides requirements relevant to the interconnection and interoperability between power systems and distributed energy resources, there is still limited knowledge or contradictory conclusions regarding the behaviour of converter-based distributed energy resources during faults on transmission or distribution systems. Full-scale static converters effectively isolate the electrical generator and its specific electrical performance characteristics from the power system in the event of a fault on the host system [5]. Many modern static inverters tend to use insulated gate bipolar transistor (IGBT) switches. The performance of series converter-connected generators during a fault on the converter output terminals or elsewhere on the power system is dictated by the control strategy of the inverter. Before a fault occurs, the inverter output current is controlled both in magnitude and phase. The phase is typically set within minimum and maximum limits with respect to the zero crossing of the output voltage to ensure that the inverter reactive power output is kept within corresponding minimum and maximum limits. When a symmetrical three-phase fault occurs on the power system, the inverter's inherent constant current control strategy can ensure that the inverter continues to supply the same current as that supplied in the power frequency cycle just before the occurrence of the fault. The magnitude of the inverter fault current contribution due to the inherent constant current control strategy in the event of a symmetrical three-phase fault at bus $i$ can be illustrated as shown on the next page in Figure 4.2. 
(a) Series converter-connected wind turbine generator, synchronous or induction

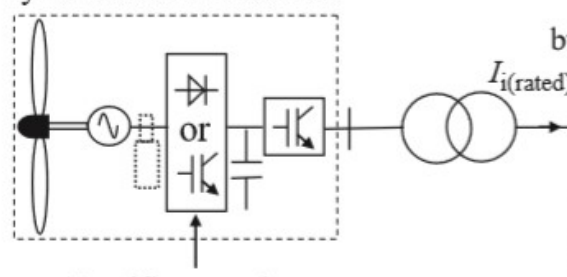

Rectifier or voltage source converter

(b)

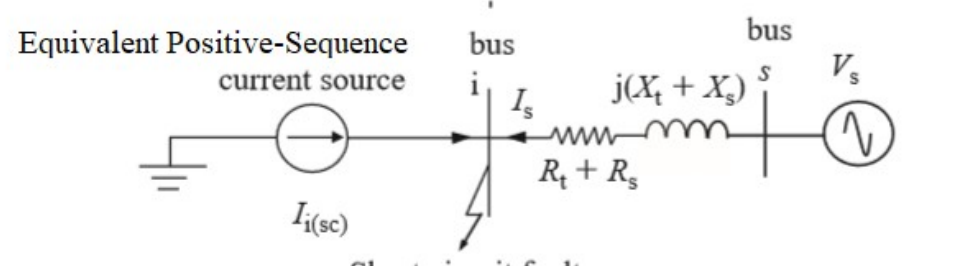

Short circuit fault

(c)

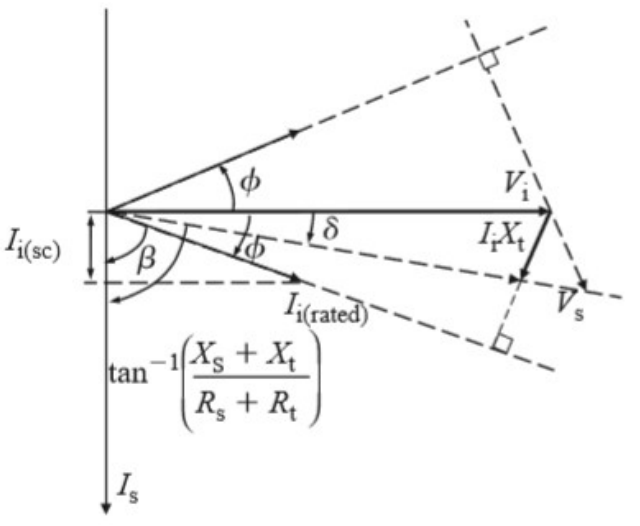

Figure 4.2: Modelling the fault current contribution of a series converter-connected wind turbine generator: (a) Initial prefault condition; (b) Fault condition; and (c) Vector diagram under fault conditions [5]. 


\subsection{Solution Method}

\subsubsection{Fault Analysis of Converter-Based Distributed Energy Resources for AC Side Bolted Faults}

Figure 4.2(a) on the previous page shows a series converter-connected wind turbine generator, which may be induction or synchronous, with the grid-side inverter connected through a transformer to a distribution system voltage level (typically either $13.8 \mathrm{kV}$ or $27.6 \mathrm{kV}$ in Ontario). This is then connected to a higher voltage grid system through another transformer. The largest prefault inverter current is that supplied at rated power and reactive power output of the inverter. Assume that at bus $i$ the inverter rated power output is $P$, rated lagging reactive power output is $Q$ and the rated lagging power factor is $\cos \phi$. Therefore, the rated inverter current delivered at bus $i$ just before the occurrence of the fault is given by the equation

$$
\mathbf{I}_{i(\text { rated })}=\frac{P-j Q}{\mathbf{V}_{i}^{*}}=\left|\mathbf{I}_{i(\text { rated })}\right| e^{-j \phi}
$$

The relationship between the voltage $\mathbf{V}_{i}$ at bus $i$ and the grid system voltage $\mathbf{V}_{s}$ at bus $s$, neglecting the transformer resistance $R_{t}$ as a simplifying assumption because it is much smaller than its reactance $X_{t}$, is given by the equation

$$
\mathbf{V}_{s}=\mathbf{V}_{i}-j X_{t} \mathbf{I}_{i(\text { rated })}=\left|\mathbf{V}_{s}\right| e^{-j \delta}
$$

At bus $s$, the grid system is represented by its Thevenin equivalent circuit. Since the grid-side inverter continues to supply a constant, balanced three-phase current during the fault and the resultant voltage dip at its terminals, the inverter and its transformer can be represented as a constant positive-sequence current source as shown on the previous page in Figure 4.2(b). For a symmetrical three-phase fault at bus $i$, the positive-sequence current supplied to the fault from the high voltage grid system is given by the equation

$$
\mathbf{I}_{s}=\frac{\mathbf{V}_{s}}{Z_{s}+Z_{t}}=\frac{\mathbf{V}_{s}}{\sqrt{\left(R_{S}+R_{t}\right)^{2}+\left(X_{S}+X_{t}\right)^{2}}} e^{-j \tan ^{-1}\left[\left(X_{S}+X_{t}\right) /\left(R_{S}+R_{t}\right)\right]}
$$

In practice, $\left(X_{S}+X_{t}\right) /\left(R_{s}+R_{t}\right)$ will be dominated by the transformer $X_{t} / R_{t}$ ratio, which is typically between 14 and 30 [5] but may be as high as 45 [14], [15] depending on the rating of the transformer as mentioned earlier. A transformer $X_{t} / R_{t}$ ratio of 14 and 30 correspond to impedance phase angles $\tan ^{-1}\left(X_{t} / R_{t}\right)$ of $86^{\circ}$ and $88^{\circ}$, respectively. Figure 4.2 (c) on the previous page shows the vector diagram of the above voltages and currents including the current supplied to the fault from the high voltage grid 
system. The component of positive-sequence current supplied to the fault from the inverter that is in phase with $\mathbf{I}_{S}$ is given by the equation

$$
\left|\mathbf{I}_{i(\mathrm{sc})}\right|=\left|\mathbf{I}_{i(\text { rated })}\right| \cos \beta=\left|\mathbf{I}_{i(\text { rated })}\right| \cos \left[\tan ^{-1}\left(\frac{X_{t}}{R_{t}}\right)-(\phi-\delta)\right]
$$

For example, for $X_{t} / R_{t}=14.3$ or $\tan ^{-1}\left(X_{t} / R_{t}\right)=86^{\circ}$, rated inverter lagging power factor on the high voltage side of its transformer equal to 0.95 or $\phi=\cos ^{-1}(0.95)=18.2^{\circ}$, and load angle $\delta=6^{\circ}$, it can be observed from Equation (4.4) that

$$
\left|\mathbf{I}_{i(\mathrm{sc})}\right|=\left|\mathbf{I}_{i(\text { rated })}\right| \cos \left[86^{\circ}-\left(18.2^{\circ}-6^{\circ}\right)\right] \approx 0.28\left|\mathbf{I}_{i(\text { rated })}\right|
$$

The total fault current at bus $i$ is simply the sum of the high voltage grid system and inverter fault current contributions, i.e., $\mathbf{I}_{f, i}=\mathbf{I}_{s}+\mathbf{I}_{i(\mathrm{sc})}$.

If the inverter were operating at rated leading power factor, it can be similarly shown that

$$
\left|\mathbf{I}_{i(\mathrm{sc})}\right|=\left|\mathbf{I}_{i(\text { rated })}\right| \cos \left[\tan ^{-1}\left(\frac{X_{t}}{R_{t}}\right)-(\phi+\delta)\right]
$$

However, in this case, although the current supplied to the fault from the inverter has a larger magnitude, it is in anti-phase with the dominate current supplied to the fault from the high voltage grid system and will therefore act to reduce the total fault current at bus $i$. Equation (4.4) shows that the inherent inverter fault current contribution in the event of a symmetrical three-phase fault at bus $i$ is a small fraction of the inverter rated current for a constant current control strategy which ensures that the inverter continues to supply the same current (both in magnitude and phase) during the fault as that supplied in the power frequency cycle just before the occurrence of the fault, i.e., $\mathbf{I}_{i(\mathrm{rated})}$.

Series converter-connected generators with a grid-side inverter can provide a controlled fault current response during the entire fault duration [5]. Therefore, utilities typically require a larger fault current contribution from such generators to assist power system protection in the detection of fault currents. As a result, modern grid-side inverter controls are typically designed to supply a larger and constant value of three-phase fault current that is related to the rated current by

$$
\left|\mathbf{I}_{i(\mathrm{sc})}\right|=\alpha\left|\mathbf{I}_{i(\text { rated })}\right|
$$


where $\alpha \leq 3$ for most current inverter designs [5] - [12]. The inverter can be considered to act as a positivesequence constant current source during the entire fault duration as given by Equation (4.7). In addition, the inverter constant current control strategy ensures that the positive-sequence fault current supplied does not contain a dc component of current [5].

Under unbalanced fault conditions, e.g., single line-to-ground fault, the control systems of most modern inverters are designed to continue to supply balanced three-phase currents irrespective of the degree of their voltage unbalance. Therefore, under unbalanced fault conditions, the inverter will only supply a positive-sequence current to the fault with the negative- and zero-sequence currents being equal to zero [5]. In other words, series converter-connected generators with grid-side inverters (e.g., solar PV and wind turbine generators) that deliver an increased constant current in response to the occurrence of a fault on the power system can be represented in industry-based fault studies by a positive-sequence equivalent circuit consisting of a constant current source, given by Equation (4.7), and negative- and zero-sequence equivalent circuits consisting of infinite negative- and zero-sequence impedances, respectively, i.e., opencircuits. To overcome the difficulty of integrating a constant current source into the framework of conventional fault analysis, it must be replaced by a voltage source behind an inductive reactance (as in the case of synchronous generators) using a Norton-Thevenin source transformation such that the conventional fault current calculation techniques described earlier in Chapters 2 and 3 may be employed. However, it is generally incorrect to assume that a series converter-connected generator with a grid-side inverter can be modeled by an internal generated voltage $\mathbf{E}_{A}$ behind a positive-sequence impedance $Z_{1}$ such that $\left|\mathbf{I}_{i(\mathrm{sc})}\right|=\left|\mathbf{E}_{A}\right| /\left|Z_{1}\right|$. This representation will produce the correct inverter fault current contribution for one fault location only, namely at the inverter transformer output terminals. At other fault locations on the power system, the source transformation will produce a lower and incorrect inverter fault current contribution than that given by Equation (4.7). Since the equivalent positive-sequence impedance varies with the inverter transformer terminal voltage which is a function of the fault current, the equivalent positivesequence impedance must be determined by an iterative process to obtain the correct inverter fault current contribution (as will be seen in Chapter 5). Furthermore, the inverter constant current control strategy is normally very fast so that the ac symmetrical component of current does not change with time during the fault, which means that the subtransient and steady-state inverter fault current contributions are equal [5]. 


\subsubsection{Fault Analysis of Converter-Based Distributed Energy Resources for DC Side Faults}

The discussion thus far had been limited to modelling and fault analysis of converter-based distributed energy resources in the event of a fault on the host system. The discussion will now be extended to include modelling and fault analysis of converter-based distributed energy resources in the event of a fault on the dc side of a grid-side inverter. The calculation of fault currents in dc systems is essential for the design and application of distribution and protective apparatuses used in these systems. Although different sources of dc fault currents may be considered, the focus of this discussion will be on battery power applications, which are commonly used as energy storage systems in conjunction with solar PV generation systems. Though the simplified procedures for dc fault current calculations are documented in some publications, these are not well established [14], [15]. There is no American National Standards Institute (ANSI)/Institute of Electrical and Electronics Engineers (IEEE) standard for the calculation of fault currents in dc systems. A General Electric Company publication [20] and IEEE Std C37.14-2015 [21] provide some guidelines. The International Electrotechnical Commission (IEC) standard 61660-1 [22], published in 1997, is the only comprehensive document available on this subject. This standard addresses the calculation of fault currents in dc auxiliary installations in power plants and substations and does not include calculations in other large dc power systems, such as electrical railway traction and transit systems. Analogous to fault current calculations in ac systems, the maximum possible fault currents must be considered for selecting the withstand rating of all electrical equipment as they must be designed to withstand this extremely high current level for a brief period without damage. To determine the maximum possible fault currents that a dc system must be able to withstand without being destroyed, it is assumed that the battery is fully charged and the fault current contribution of the converter is limited to a pre-set maximum value due to the current control capability of modern converter control systems, which remove the firing pulses to the IGBTs when the current exceeds a particular threshold in order to preserve the electronic components [6], [12], [14], [15]. Note that the ac system contributes to a dc fault current only when the converter operates in rectifier mode, and the fault current contribution only becomes significant when the rectifier is either without grid control (such converters have become obsolete) or the controls for limiting the rectifier current are not effective. Therefore, in the case of solar PV generation with battery bank storage connected through a grid-side inverter, only the fault current contribution of the storage batteries to a fault on the dc side of the grid-side inverter will be considered since the ac system will not contribute to the dc fault current when the converter operates in inverter mode. The remainder of this discussion will consider the fault current contribution of fully charged lead-acid storage batteries during a fault on the dc side of a grid-side inverter by comparing the results of two different approaches for calculating the dc fault current, beginning with the guidelines

provided in the General Electric Company publication [20] subsequently followed by the procedures outlined in IEC standard 61660-1 [22]. 
The equivalent circuit of a lead-acid storage battery for evaluating fault current is shown below in Figure 4.3 and consists of a voltage source $\left(E_{\mathrm{B}}\right)$, two resistances $\left(R_{\mathrm{B}}\right.$ and $\left.R_{\mathrm{C}}\right)$, and an inductance $\left(L_{\mathrm{C}}\right)$. The magnitude of the voltage source is determined based on two volts per cell or

$$
E_{\mathrm{B}}=2.0 \mathrm{~V} / \text { cell } \times \text { number of cells }(\mathrm{V})
$$

One of the resistances in the equivalent circuit of Figure 4.3 represents the internal resistance $\left(R_{\mathrm{B}}\right)$ of the battery and its magnitude is equal to the battery internal voltage ( $2 \mathrm{~V}$ per cell) divided by 100 times the 8hour ampere rating of the battery, i.e.,

$$
R_{\mathrm{B}}=\frac{E_{\mathrm{B}}}{100 I_{8 \mathrm{HR}}}(\Omega)
$$

The other resistance $\left(R_{\mathrm{C}}\right)$ in the equivalent circuit of Figure 4.3 represents the conductors which connect the cells together. The magnitude of this resistance must be based on a knowledge of the conductor size, length, and composition. The inductance $\left(L_{\mathrm{C}}\right)$ in the equivalent circuit of Figure 4.3 represents the inductance of the circuit which is created by the conductors connecting the cells together. The cells themselves are considered to have no internal inductance. The physical arrangement of a 60-cell lead-acid storage battery mounted in three rows is shown on the next page in Figure 4.4.

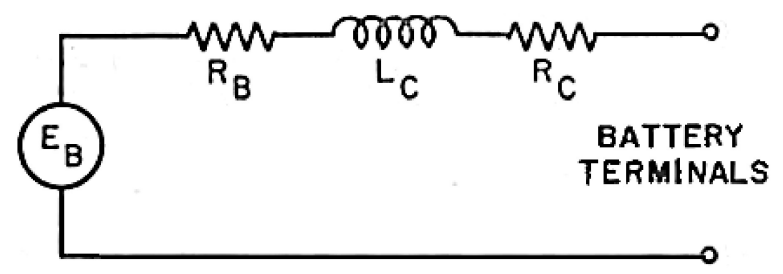

$$
\begin{aligned}
& R_{\mathrm{B}}=\text { Battery internal resistance }=\frac{E_{\mathrm{B}}}{100 I_{8 \mathrm{HR}}}(\Omega) \\
& E_{\mathrm{B}}=\text { Battery internal voltage }=2.0 \mathrm{~V} / \text { cell } \times \text { number of cells }(\mathrm{V}) \\
& R_{\mathrm{C}}=\text { Resistance of cell connectors }(\Omega) \\
& L_{\mathrm{C}}=\text { Inductance of cell circuit }(\mathrm{H}) \\
& I_{8 \mathrm{HR}}=\text { 8-hour ampere rating of battery }(\mathrm{A})
\end{aligned}
$$

Figure 4.3: Equivalent circuit of battery for calculating fault current [20]. 


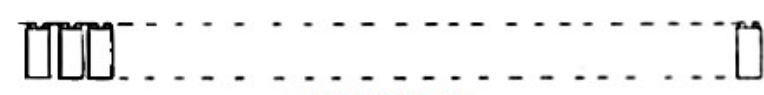

FRONT VIEW

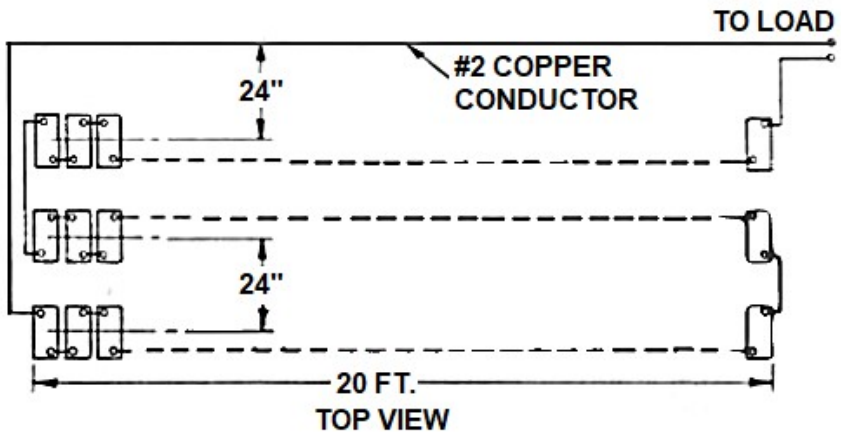

Figure 4.4: Physical arrangement of 60-cell lead-acid storage battery mounted in three rows [20].

The differential equation describing the equivalent circuit of Figure 4.3 is given by

$$
i_{\mathrm{B}}\left(R_{\mathrm{B}}+R_{\mathrm{C}}\right)+L_{\mathrm{C}} \frac{d i_{\mathrm{B}}}{d t}=E_{\mathrm{B}}
$$

The solution of this equation is given by

$$
i_{\mathrm{B}}=\frac{E_{\mathrm{B}}}{\left(R_{\mathrm{B}}+R_{\mathrm{C}}\right)}\left[1-e^{-\left(\left(R_{\mathrm{B}}+R_{\mathrm{C}}\right) / L_{\mathrm{C}}\right) t}\right](\mathrm{A})
$$

The maximum fault current can be calculated from the equation

$$
I_{\mathrm{B}(\mathrm{SC})}=\frac{E_{\mathrm{B}}}{R_{\mathrm{B}}+R_{\mathrm{C}}}(\mathrm{A})
$$

The initial maximum rate of rise of the fault current is given by $d i_{\mathrm{B}} / d t$ at $t=0$ seconds, i.e.,

$$
\frac{d i_{\mathrm{B}}}{d t}=\frac{E_{\mathrm{B}}}{L_{\mathrm{C}}}(\mathrm{A} / \mathrm{s})
$$

The fault current vs. time profile for the battery will be a simple exponential curve since all the circuit parameters remain constant during the transient. 
As an example of the guidelines provided in the General Electric Company publication [20] outlined above, consider the 60-cell lead-acid storage battery mounted in three rows as shown on the previous page in Figure 4.4. The battery is rated $200 \mathrm{Ah}$ (ampere hour) based on an 8-hour rate of discharge to $1.75 \mathrm{~V}$ per cell at $20^{\circ} \mathrm{C}$. The battery internal voltage according to Equation (4.8) is

$$
E_{\mathrm{B}}=2.0 \mathrm{~V} / \text { cell } \times 60 \text { cells }=120 \mathrm{~V}
$$

The battery internal resistance according to Equation (4.9) is

$$
R_{\mathrm{B}}=\frac{120 \mathrm{~V}}{100 \times 25 \mathrm{~A}}=0.048 \Omega
$$

The cell connections consist of a total of approximately 7.9248 meters (26 feet) of No. 2 copper conductor:

$$
R_{\mathrm{C}}=7.9248 \mathrm{~m} \times 0.162 \mathrm{ohms} / 304.8 \mathrm{~m} \times \frac{1}{304.8}=0.004 \Omega
$$

The inductance of the cell circuit is determined from the configuration shown previously in Figure 4.4. The total conductor length is approximately 26.2128 meters ( 86 feet). The battery cells constitute 18.288 meters (60 feet) of this conductor length and the No. 2 copper conductor constitutes the remaining 7.9248 meters (26 feet). The circuit inductance can be determined by referring to appropriate 60 -cycle reactance data and dividing the inductive reactance by $2 \pi f=2 \pi(60 \mathrm{~Hz})=377 \mathrm{rad} / \mathrm{s}$. To calculate the inductance for the battery cells, assume that the cells are the equivalent of a $15.24 \mathrm{~cm}$ (six-inch) conductor spaced $60.96 \mathrm{~cm}$ (24 inches) from the return conductor. The 60-cycle reactance data shown on the next page in Figure 4.5 for bus-bar circuits is sufficiently accurate for representing the battery cells. From Figure 4.5, for $15.24 \mathrm{~cm}$ (six-inch) channels spaced $60.96 \mathrm{~cm}$ (24 inches) apart:

$$
\begin{aligned}
& X=160.76 \times 10^{-6} \Omega / \mathrm{m} \\
& L=\frac{160.76 \times 10^{-6} \Omega / \mathrm{m}}{377 \mathrm{rad} / \mathrm{s}}=0.4264 \times 10^{-6} \mathrm{H} / \mathrm{m} \\
& L=18.288 \mathrm{~m} \times 0.4264 \times 10^{-6} \mathrm{H} / \mathrm{m}=7.8 \times 10^{-6} \mathrm{H}
\end{aligned}
$$

Similarly, the 60 -cycle reactance data shown on page 77 in Figure 4.6 can be used to represent the No. 2 copper conductor on $60.96 \mathrm{~cm}$ (24-inch) spacing:

$$
X=0.126 \Omega / 304.8 \mathrm{~m}
$$




$$
\begin{aligned}
& L=\frac{0.126 \Omega / 304.8 \mathrm{~m}}{377 \mathrm{rad} / \mathrm{s}}=0.00033 \mathrm{H} / 304.8 \mathrm{~m} \\
& L=7.9248 \mathrm{~m} \times 0.00033 \mathrm{H} / 304.8 \mathrm{~m} \times \frac{1}{304.8}=8.6 \times 10^{-6} \mathrm{H}
\end{aligned}
$$

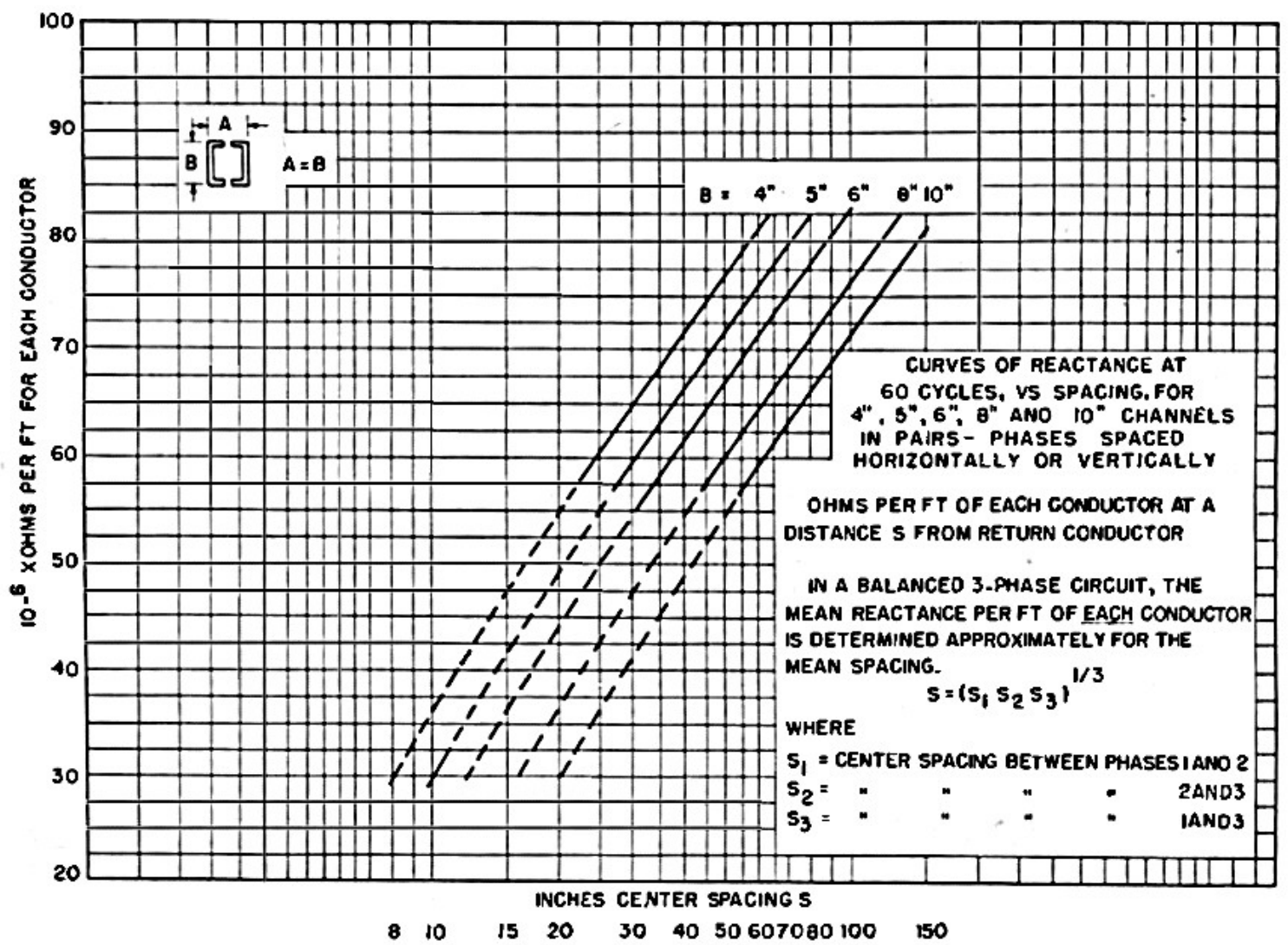

Figure 4.5: Bus-bar reactance in ohms per 0.3048 meters (per foot) for each conductor at 60 cycles [20]. 


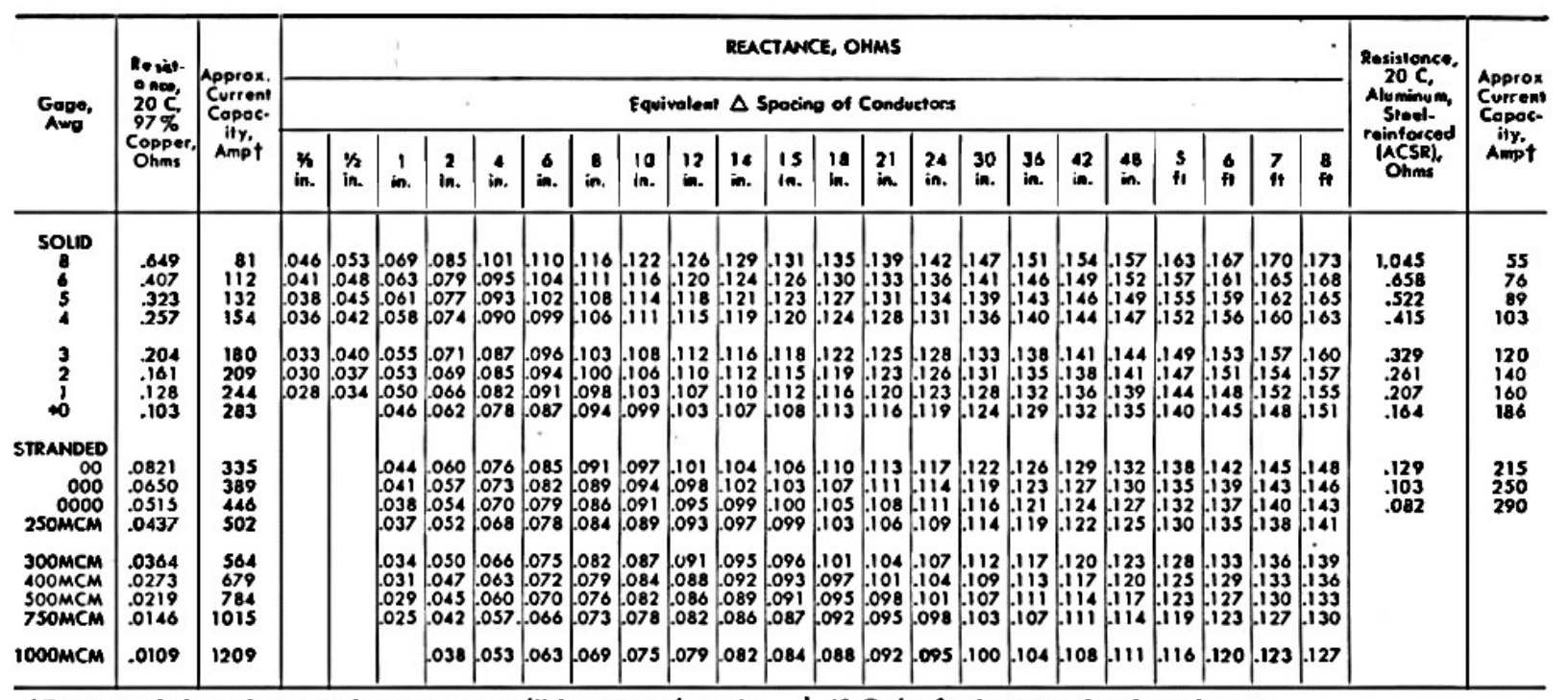

"For stranded conductors, the reactance will be approximately $† 40 \mathrm{C}$ rise for bare overhead conductor.

$0.001 \mathrm{ohm}$ less than for solid conductors of the same size. Reference-Overhead Systems Reference Book-1927.

Figure 4.6: Transmission line resistance and reactance per single conductor in ohms per 304.8 meters (1000 feet) at 60 cycles [20].

The total inductance for the battery circuit is then equal to

$$
L_{C}=(7.8+8.6) \times 10^{-6} \mathrm{H}=16.4 \times 10^{-6} \mathrm{H}
$$

The equivalent circuit for this battery appears as shown on the next page in Figure 4.7. The maximum fault current according to Equation (4.12) is

$$
I_{\mathrm{B}(\mathrm{SC})}=\frac{120 \mathrm{~V}}{0.048 \Omega+0.004 \Omega}=2310 \mathrm{~A}
$$

The initial maximum rate of rise of the fault current according to Equation (4.13) is

$$
\frac{d i_{\mathrm{B}}}{d t}=\frac{120 \mathrm{~V}}{16.4 \times 10^{-6} \mathrm{H}}=7.3 \times 10^{6} \mathrm{~A} / \mathrm{s}
$$

The fault current-time profile for this example is shown on the next page in Figure 4.8. The time constant for this circuit is equal to

$$
\frac{L_{\mathrm{C}}}{R_{\mathrm{B}}+R_{\mathrm{C}}}=\frac{16.4 \times 10^{-6} \mathrm{H}}{0.048 \Omega+0.004 \Omega}=0.32 \mathrm{~ms}
$$


In other words, the fault current will equal $63 \%$ of $2310 \mathrm{~A}$ (or equivalently $1450 \mathrm{~A}$ ) at 0.32 milliseconds after the fault is initiated.

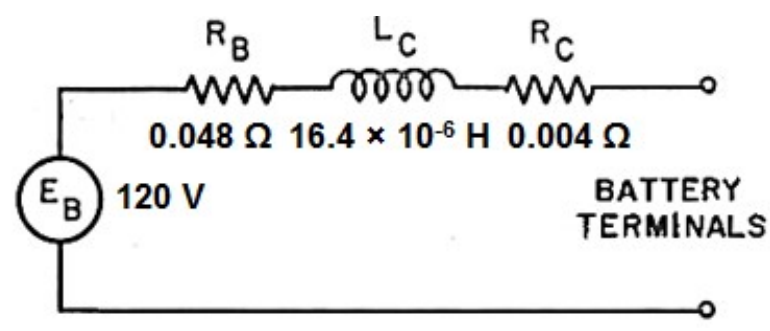

Figure 4.7: Equivalent circuit for example calculation [20].

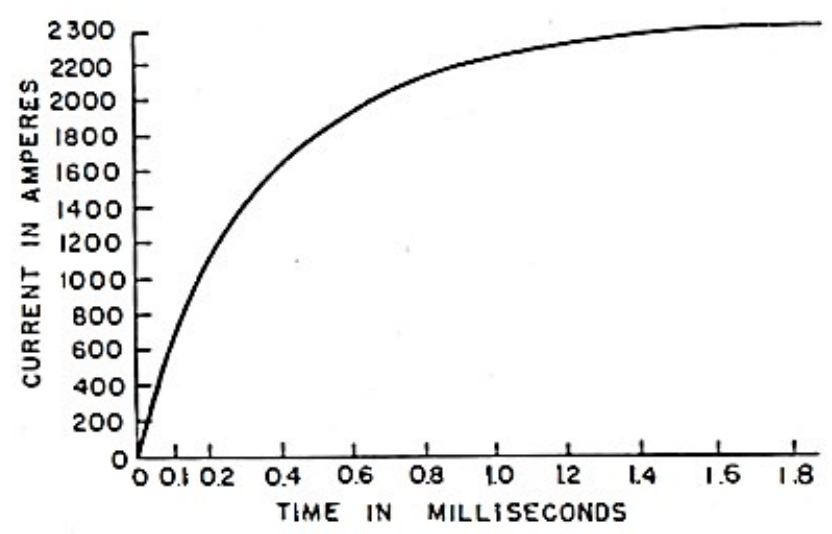

Figure 4.8: Fault current-time profile of battery for example calculation [20].

The typical fault current-time profile of a lead-acid storage battery and the standard approximate functions as per IEC standard 61660-1 [22] are both shown on the next page in Figures 4.9 and 4.10, respectively. The following definitions apply to the procedures outlined in IEC standard 61660-1 [22]:

$$
\begin{aligned}
& I_{\mathrm{kB}}=\text { quasi steady-state fault current }(\mathrm{A}) \\
& i_{\mathrm{pB}}=\text { peak fault current }(\mathrm{A}) \\
& T_{\mathrm{kB}}=\text { fault duration }(\mathrm{s}) \\
& t_{\mathrm{pB}}=\text { time to peak }(\mathrm{s}) \\
& \tau_{1 \mathrm{~B}}=\text { rise time constant }(\mathrm{s}) \\
& \tau_{2 \mathrm{~B}}=\text { decay time constant }(\mathrm{s})
\end{aligned}
$$

The standard approximate functions shown on the next page in Figure 4.10 are described by the following equations: 


$$
\begin{gathered}
i_{1}(t)=i_{\mathrm{pB}} \frac{1-e^{-t / \tau_{1 \mathrm{~B}}}}{1-e^{-t_{\mathrm{pB}} / \tau_{1 \mathrm{~B}}}}(\mathrm{~A}) \\
i_{2}(t)=i_{\mathrm{pB}}\left[(1-\alpha) e^{-\left(t-t_{\mathrm{pB}}\right) \tau_{2 \mathrm{~B}}}+\alpha\right](\mathrm{A}), t \geq t_{\mathrm{pB}} \\
\alpha=I_{\mathrm{kB}} / i_{\mathrm{pB}}
\end{gathered}
$$

The quasi steady-state current, $I_{\mathrm{kB}}$, is conventionally assumed as the value at 1 second after the fault is initiated.

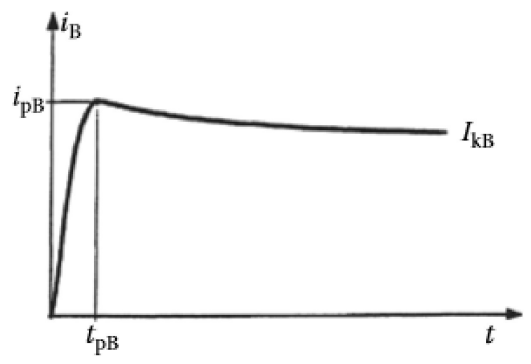

Figure 4.9: Fault current-time profile of battery [22].

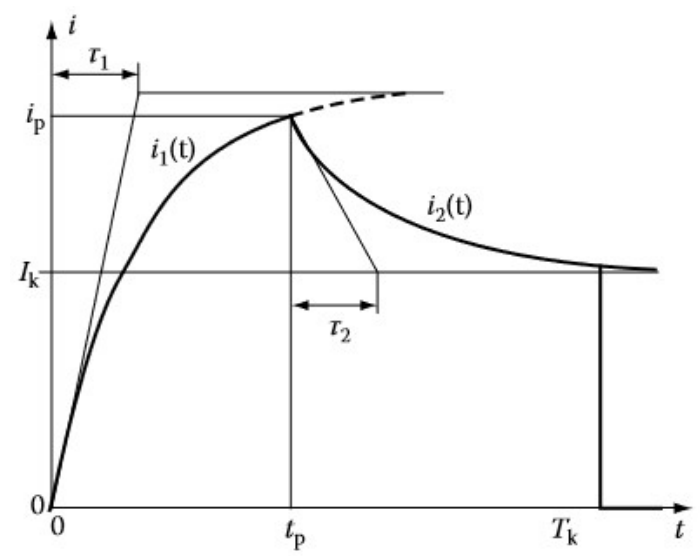

Figure 4.10: Standard approximation of fault function [22].

To calculate the maximum fault current or the peak current according to IEC standard 61660-1 [22], the battery internal resistance $R_{\mathrm{B}}$ is multiplied by a factor of 0.9 . All other impedances shown previously in Figure 4.3 remain unchanged. Also, if the open-circuit voltage of the battery is unknown, then the battery internal voltage $E_{\mathrm{B}}=1.05 U_{\mathrm{nB}}$ is used, where $U_{\mathrm{nB}}=2.0 \mathrm{~V} /$ cell for lead-acid batteries. The peak fault current is given by the equation 


$$
i_{\mathrm{pB}}=\frac{E_{\mathrm{B}}}{R_{\mathrm{BBr}}}(\mathrm{A})
$$

where $i_{\mathrm{pB}}$ is the peak fault current from the battery and $R_{\mathrm{BBr}}$ is the total equivalent resistance up to the fault location in the battery circuit, with $R_{\mathrm{B}}$ multiplied by a factor of 0.9 , i.e., $R_{\mathrm{BBr}}=0.9 R_{\mathrm{B}}+R_{\mathrm{C}}(\Omega)$. The time to peak and the rise time constant are read from the curves shown on the next page in Figure 4.11, based on $1 / \delta$, which is defined by the equation

$$
\frac{1}{\delta}=\frac{2}{\frac{R_{\mathrm{BBr}}}{L_{\mathrm{BBr}}}+\frac{1}{T_{\mathrm{B}}}}(\mathrm{s})
$$

The time constant $T_{\mathrm{B}}$ is specified as equal to 30 milliseconds and $L_{\mathrm{BBr}}$ is the total equivalent inductance up to the fault location in the battery circuit. The decay time constant $\tau_{2 \mathrm{~B}}$ is considered to be 100 milliseconds. The quasi steady-state fault current is given by the equation

$$
I_{\mathrm{kB}}=\frac{0.95 E_{\mathrm{B}}}{R_{\mathrm{BBr}}+0.1 R_{\mathrm{B}}}(\mathrm{A})
$$

Note that Equation (4.19) considers that the battery voltage falls and the internal cell resistance increases after the occurrence of a fault (a discharged battery will have a higher cell resistance).

As an example of the procedure outlined in IEC standard 61660-1 [22], consider the same 60-cell lead-acid storage battery mounted in three rows used in the previous example. The total equivalent resistance in the battery circuit is $R_{\mathrm{BBr}}=0.9 R_{\mathrm{B}}+R_{\mathrm{C}}=0.9 \times 0.048+0.004=0.0472 \Omega$. The battery nominal voltage of $120 \mathrm{~V}$ is multiplied by a factor of 1.05 . Therefore, the peak fault current according to Equation (4.17) is

$$
i_{\mathrm{pB}}=\frac{1.05 \times 120 \mathrm{~V}}{0.0472 \Omega}=2670 \mathrm{~A}
$$

This is approximately $15.6 \%$ higher compared to the calculation of the previous example. From Equation (4.18),

$$
\frac{1}{\delta}=\frac{2}{\frac{0.0472 \Omega}{16.4 \times 10^{-6} \mathrm{H}}+\frac{1}{30 \times 10^{-3} \mathrm{~s}}}=0.7 \mathrm{~ms}
$$


From Figure 4.11 , the time to peak $t_{\mathrm{pB}} \approx 2$ milliseconds and the rise time constant $\tau_{1 \mathrm{~B}} \approx 0.35$ milliseconds. These results are consistent with those of the previous example. The quasi steady-state fault current according to Equation (4.19) is

$$
I_{\mathrm{kB}}=\frac{0.95 \times 120 \mathrm{~V}}{0.0472 \Omega+0.1 \times 0.048 \Omega}=2192 \mathrm{~A}
$$

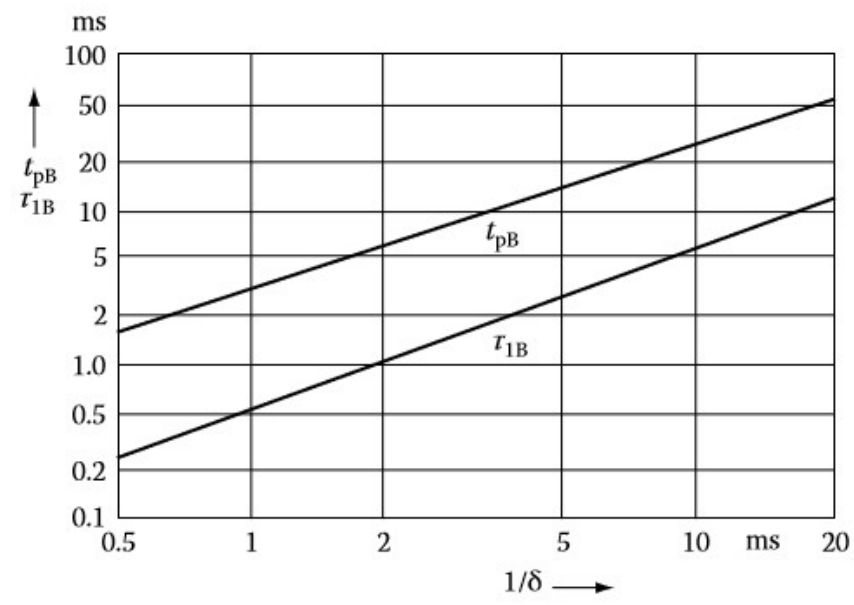

Figure 4.11: Time to peak $t_{\mathrm{pB}}$ and rise time constant $\tau_{1 \mathrm{~B}}$ for fault of a battery [22]. 


\subsection{Chapter Summary}

Series converter-connected generators with a grid-side inverter effectively isolate the electrical generator and its specific electrical performance characteristics from the power system in the event of a fault on the host system and can provide a controlled fault current response during the entire fault duration [5]. Therefore, utilities typically require a larger fault current contribution from such generators to assist power system protection in the detection of fault currents. As a result, modern grid-side inverter controls are typically designed to supply a larger and constant value of three-phase fault current that is related to the rated current by

$$
\left|\mathbf{I}_{i(\mathrm{sc})}\right|=\alpha\left|\mathbf{I}_{i(\text { rated })}\right|
$$

where $\alpha \leq 3$ for most current inverter designs [5] - [12]. The inverter can be considered to act as a positivesequence constant current source during the entire fault duration as given by Equation (4.7). In addition, the inverter constant current control strategy ensures that the positive-sequence fault current supplied does not contain a dc component of current [5].

Under unbalanced fault conditions, e.g., single line-to-ground fault, the control systems of most modern inverters are designed to continue to supply balanced three-phase currents irrespective of the degree of their voltage unbalance. Therefore, under unbalanced fault conditions, the inverter will only supply a positive-sequence current to the fault with the negative- and zero-sequence currents being equal to zero [5]. In other words, series converter-connected generators with grid-side inverters (e.g., solar PV and wind turbine generators) that deliver an increased constant current in response to the occurrence of a fault on the power system can be represented in industry-based fault studies by a positive-sequence equivalent circuit consisting of a constant current source, given by Equation (4.7), and negative- and zero-sequence equivalent circuits consisting of infinite negative- and zero-sequence impedances, respectively (i.e., opencircuits). To overcome the difficulty of integrating a constant current source into the framework of conventional fault analysis, it must be replaced by a voltage source behind an inductive reactance (as in the case of synchronous generators) using a Norton-Thevenin source transformation such that the conventional fault current calculation techniques may be employed. However, it is generally incorrect to assume that a series converter-connected generator with a grid-side inverter can be modeled by an internal

generated voltage $\mathbf{E}_{A}$ behind a positive-sequence impedance $Z_{1}$ such that $\left|\mathbf{I}_{i(\mathrm{sc})}\right|=\left|\mathbf{E}_{A}\right| /\left|Z_{1}\right|$. This representation will produce the correct inverter fault current contribution for one fault location only, namely at the inverter transformer output terminals. At other fault locations on the power system, the source transformation will produce a lower and incorrect inverter fault current contribution than that given by Equation (4.7). Since the equivalent positive-sequence impedance varies with the inverter transformer terminal voltage which is a function of the fault current, the equivalent positive-sequence impedance must 
be determined by an iterative process to obtain the correct inverter fault current contribution. Furthermore, the inverter constant current control strategy is normally very fast so that the ac symmetrical component of current does not change with time during the fault, which means that the subtransient and steady-state inverter fault current contributions are equal [5]. 


\section{Chapter 5 - MATLAB Fault Current Analysis Program}

\subsection{Introduction}

The discussion of the previous chapter identified the importance of developing mathematical and software simulation models that accurately approximate the response of converter-based distributed energy resources during a fault on the transmission or distribution system. In this chapter, a MATLAB program developed for such a purpose, i.e., calculating the voltages and fault currents at each bus and in each transmission line in the power system for three-phase, single line-to-ground, line-to-line, or double line-toground faults during the subtransient, transient, or steady-state periods, is verified and validated against published cases and other fault current analysis software programs to ensure its technical accuracy. After comparing the results, the MATLAB fault current analysis program is then used to perform fault analysis of a power system with converter-based distributed energy resources in order to simulate the fault current contributions to the electrical grid that a transmission or distribution utility needs to reflect in their connection impact assessments. The source code for the MATLAB program is provided in the appendix of this report.

The input data for the MATLAB fault current analysis program is placed in an input file, which can be created using any available text editor (e.g., notepad, wordpad, etc.). The input file may contain up to six different types of input lines used to describe the power system:

- A SYSTEM line to define the power system study name and base apparent power of the power system (in MVA).

- Two or more BUS lines to define the bus names and prefault bus voltage.

- One or more LINE lines to define the transmission lines connecting the various buses together. Note that transformers at the sending and receiving ends of transmission lines are treated additional "transmission lines".

- One or more GENERATOR lines to define the impedances of generators in the power system.

- Zero or more MOTOR lines to define the impedances of motors in the power system.

- A FAULT line to specify the location, type, and time period (subtransient, etc.) of the fault.

A sample input file is shown on the next page in Figure 5.1. This input file contains all six different types of input lines, including comment lines that begin with a $\frac{\circ}{\circ}$ symbol in column 1. 


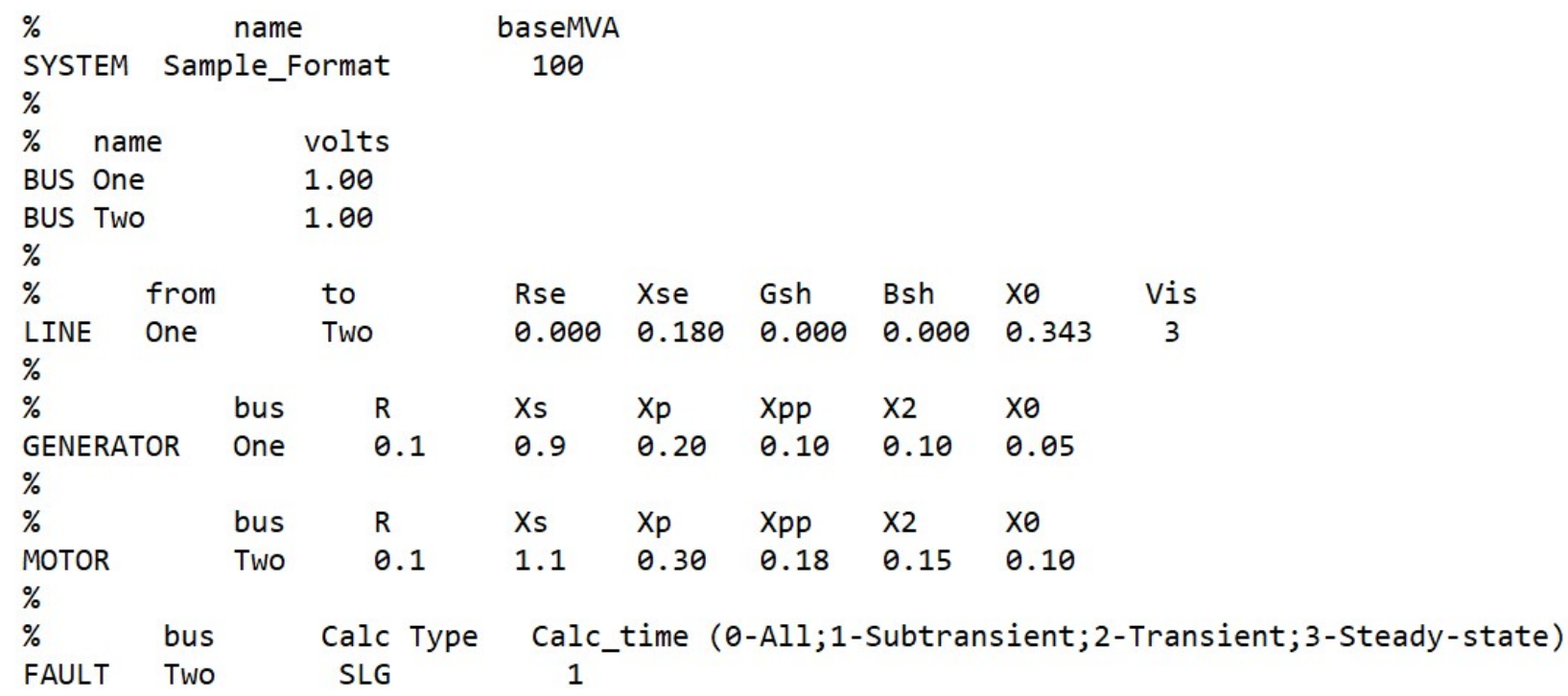

Figure 5.1: Sample input file to the MATLAB fault current analysis program.

The format of a SYSTEM line is shown below, and the values in the line are defined in Table 5.1.

$\begin{array}{lcc}\% & \text { name } & \text { baseMVA } \\ \text { SYSTEM } & \text { Sample_Format } & 100\end{array}$

\begin{tabular}{|l|l|}
\hline Field & Description \\
\hline SYSTEM & Line identifier \\
\hline name & Power system study name (spaces are not allowed within the name) \\
\hline baseMVA & Base apparent power of the power system, in MVA \\
\hline
\end{tabular}

Table 5.1: Fields defined on the SYSTEM line.

The format of a BUS line is shown below, and the values in the line are defined in Table 5.2.

$\begin{array}{ll}\% \quad \text { name } & \text { volts } \\ \text { BUS One } & 1.00\end{array}$




\begin{tabular}{|l|l|}
\hline Field & Description \\
\hline BUS & Line identifier \\
\hline name & Bus name (spaces are not allowed within the name) \\
\hline volts & Prefault bus voltage, in per unit \\
\hline
\end{tabular}

Table 5.2: Fields defined on the BUS line.

The format of a LINE line is shown below, and the values in the line are defined in Table 5.3.

$\begin{array}{llllllllc}\% & \text { from } & \text { to } & \text { Rse } & \text { Xse } & \text { Gsh } & \text { Bsh } & \text { Xo } & \text { Vis } \\ \text { LINE } & \text { One } & \text { Two } & 0.000 & 0.180 & 0.000 & 0.000 & 0.343 & 3\end{array}$

\begin{tabular}{|l|l|}
\hline Field & Description \\
\hline LINE & Line identifier \\
\hline from & Bus where transmission line begins \\
\hline to & Bus where transmission line ends \\
\hline Rse & Transmission line series resistance, in per unit \\
\hline Xse & Transmission line series reactance, in per unit \\
\hline Gsh & Transmission line shunt conductance, in per unit \\
\hline Bsh & Transmission line shunt susceptance, in per unit \\
\hline X0 & $\begin{array}{l}\text { Zero-sequence series reactance, in per unit (set to 0 for symmetrical } \\
\text { three-phase faults) }\end{array}$ \\
\hline Vis & $\begin{array}{l}\text { Zero-sequence visibility flag (set to 0 for symmetrical three-phase } \\
\text { faults): } \\
0-\text { Visible to neither bus } \\
1-\text { Visible to the "from" bus only } \\
2-\text { Visible to the "to" bus only } \\
3 \text { - Visible to both buses }\end{array}$ \\
&
\end{tabular}

Table 5.3: Fields defined on the LINE line. 
The formats of GENERATOR and MOTOR lines is shown below, and the values in the lines are defined in Table 5.4.

$\begin{array}{llllllll}\% & \text { bus } & \mathrm{R} & \mathrm{Xs} & \mathrm{Xp} & \mathrm{Xpp} & \mathrm{X} 2 & \text { X0 } \\ \text { GENERATOR } & \text { One } & 0.1 & 0.9 & 0.20 & 0.10 & 0.10 & 0.05 \\ & & & & & & & \\ \% & \text { bus } & \mathrm{R} & \mathrm{Xs} & \mathrm{Xp} & \mathrm{Xpp} & \mathrm{X} 2 & \text { X0 } \\ \text { MOTOR } & \text { TwO } & 0.1 & 1.1 & 0.30 & 0.18 & 0.15 & 0.10\end{array}$

\begin{tabular}{|l|l|}
\hline Field & Description \\
\hline GENERATOR & Line identifier \\
\hline MOTOR & Line identifier \\
\hline bus & Bus that machine is connected to \\
\hline $\mathrm{R}$ & Machine resistance, in per unit \\
\hline $\mathrm{XS}$ & Machine synchronous reactance $X_{S}$, in per unit \\
\hline $\mathrm{XP}$ & Machine transient reactance $X^{\prime}$, in per unit \\
\hline $\mathrm{XPP}$ & Machine subtransient reactance $X^{\prime \prime}$, in per unit \\
\hline $\mathrm{X} 2$ & $\begin{array}{l}\text { Negative-sequence reactance } X_{2}, \text { in per unit (set to } 0 \text { for symmetrical } \\
\text { three-phase faults) }\end{array}$ \\
\hline $\mathrm{X} 0$ & $\begin{array}{l}\text { Zero-sequence reactance } X_{0}, \text { in per unit (set to } 0 \text { for symmetrical } \\
\text { three-phase faults) }\end{array}$ \\
\hline
\end{tabular}

Table 5.4: Fields defined on the GENERATOR and MOTOR lines.

The format of a FAULT line is shown below, and the values in the line are defined in Table 5.5.

$\begin{array}{llrc}\% & \text { bus } & \text { Calc Type Calc_time ( } 0 \text {-All;1-Subtransient;2-Transient;3-Steady-state) } \\ \text { FAULT Two } & \text { SLG } & 1\end{array}$ 


\begin{tabular}{|l|l|}
\hline Field & Description \\
\hline FAULT & Line identifier \\
\hline bus & Fault location \\
\hline Cal__type & Fault type: \\
& $3 P$ - Symmetrical three-phase fault \\
& LG - Single line-to-ground fault \\
& LL - Line-to-line fault \\
& DLG - Double line-to-ground fault \\
& Time period for fault analysis: \\
& $0-$ All \\
& $1-$ Subtransient period \\
& $2-$ Transient period \\
& $3-$ Steady-state period \\
\hline
\end{tabular}

Table 5.5: Fields defined on the FAULT line.

To perform fault analysis using this MATLAB program, the per-unit impedances and admittances of all components must first be calculated to a common system base, and then an input file can be created containing all those input parameters used to describe the power system being studied. Note that transformers at the sending and receiving ends of transmission lines are treated additional "transmission lines". As mentioned earlier, the prefault voltages should be generally assumed to be a constant value (typically 1.0) to correspond to prefault no-load conditions. 


\subsection{Test Data 1}

The following is a comparison of the MATLAB simulation results against published results using PowerWorld Simulator [13]. The fault analysis is performed on a 5-bus power system whose single-line diagram is shown below in Figure 5.2. Input data are given below in Tables 5.6, 5.7, and 5.8. The input files to the MATLAB program and the corresponding MATLAB command window outputs are provided in the appendix of this report. Note that the neutrals of both transformers and the generator connected to bus 1 are solidly grounded, as indicated by a neutral reactance of zero for all these equipment. However, a neutral reactance $=0.0025$ per unit is connected to the neutral of the generator connected to bus 3 . The prefault voltage is 1.05 per unit. The fault is a single line-to-ground fault at Bus 1, then Bus 2, and so on to Bus 5. The comparison between the MATLAB simulation results and those published using PowerWorld Simulator [13] are shown on the next page in Table 5.9. Note that the results are identical with zero percent error.

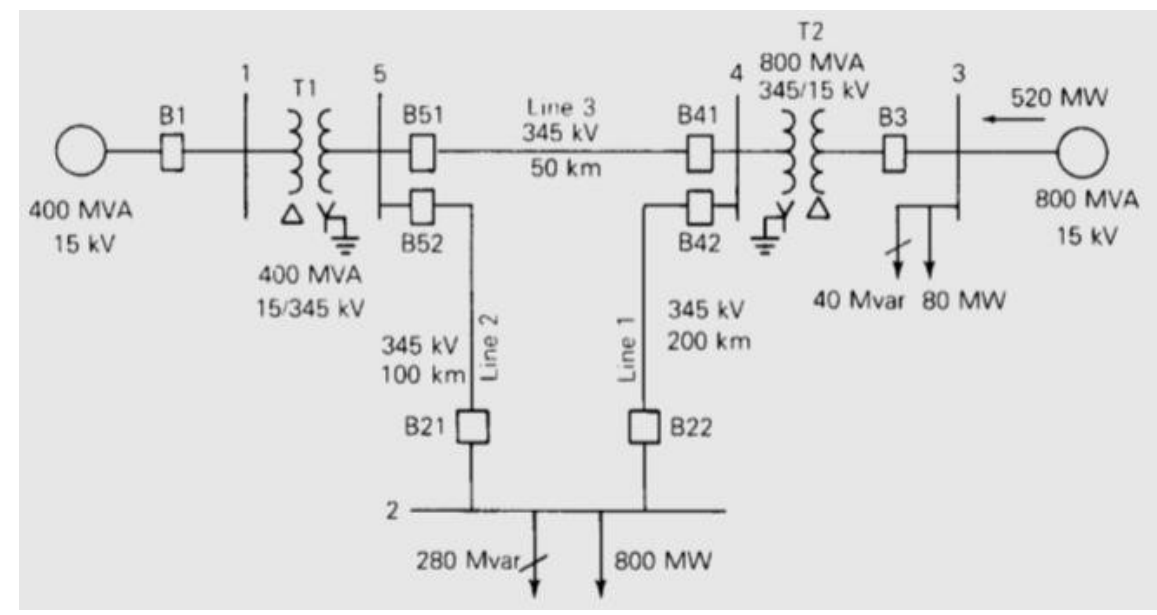

Figure 5.2: Single-line diagram for test data 1 [13].

\begin{tabular}{|l|c|c|c|c|}
\hline Bus & $\begin{array}{c}\boldsymbol{X}_{1}=\boldsymbol{X}^{\prime \prime} \\
\text { per unit }\end{array}$ & $\begin{array}{c}\boldsymbol{X}_{2} \\
\text { per unit }\end{array}$ & $\begin{array}{c}\boldsymbol{X}_{0} \\
\text { per unit }\end{array}$ & $\begin{array}{c}\text { Neutral Reactance } \boldsymbol{X}_{n} \\
\text { per unit }\end{array}$ \\
\hline 1 & 0.0450 & 0.0450 & 0.0125 & 0.0000 \\
\hline 3 & 0.0225 & 0.0225 & 0.0050 & 0.0025 \\
\hline
\end{tabular}

Table 5.6: Synchronous machine data for test data 1 (p.u., 100MVA) [13]. 


\begin{tabular}{|l|c|c|}
\hline Bus-to-Bus & $\begin{array}{c}X_{1}=X_{2} \\
\text { per unit }\end{array}$ & $\begin{array}{c}X_{0} \\
\text { per unit }\end{array}$ \\
\hline $2-4$ & 0.1000 & 0.3000 \\
\hline $2-5$ & 0.0500 & 0.1500 \\
\hline $4-5$ & 0.0250 & 0.0750 \\
\hline
\end{tabular}

Table 5.7: Transmission line data for test data 1 (p.u., 100MVA) [13].

\begin{tabular}{|l|c|c|c|c|}
\hline Bus & $\begin{array}{c}\text { Low-Voltage } \\
\text { (connection) } \\
\text { bus }\end{array}$ & $\begin{array}{c}\text { High-Voltage } \\
\text { (connection) } \\
\text { bus }\end{array}$ & $\begin{array}{c}\text { Leakage Reactance } \\
\text { per unit }\end{array}$ & $\begin{array}{c}\text { Neutral Reactance } \\
\text { per unit }\end{array}$ \\
\hline 1 & $1(\Delta)$ & $5(\mathrm{Y})$ & 0.0200 & 0.0000 \\
\hline 3 & $3(\Delta)$ & $4(\mathrm{Y})$ & 0.0100 & 0.0000 \\
\hline
\end{tabular}

Table 5.8: Transformer data for test data 1 (p.u., 100MVA) [13].

\begin{tabular}{|c|c|c|c|c|c|c|}
\hline $\begin{array}{l}\text { Fault } \\
\text { Bus }\end{array}$ & $\begin{array}{c}\text { PowerWorld } \\
\text { Single } \\
\text { Line-to- } \\
\text { Ground } \\
\text { Fault Current } \\
\text { (Phase A) } \\
\text { per unit }\end{array}$ & $\begin{array}{c}\text { MATLAB } \\
\text { Single } \\
\text { Line-to- } \\
\text { Ground } \\
\text { Fault Current } \\
\text { (Phase A) } \\
\text { per unit }\end{array}$ & $\%$ Error & $\begin{array}{c}\text { MATLAB } \\
\text { Positive- } \\
\text { Sequence } \\
\text { Thevenin } \\
\text { Equivalent } \\
\text { Impedance } \\
\text { per unit }\end{array}$ & $\begin{array}{c}\text { MATLAB } \\
\text { Negative- } \\
\text { Sequence } \\
\text { Thevenin } \\
\text { Equivalent } \\
\text { Impedance } \\
\text { per unit }\end{array}$ & $\begin{array}{c}\text { MATLAB } \\
\text { Zero- } \\
\text { Sequence } \\
\text { Thevenin } \\
\text { Equivalent } \\
\text { Impedance } \\
\text { per unit }\end{array}$ \\
\hline 1 & 46.02 & 46.02 & 0.00 & j0.027973 & j0.027973 & $\mathrm{j} 0.012500$ \\
\hline 2 & 14.14 & 14.14 & 0.00 & j0.056952 & j0.056952 & j0.108939 \\
\hline 3 & 64.30 & 64.30 & 0.00 & j0.018243 & j0.018243 & j0.012500 \\
\hline 4 & 56.07 & 56.07 & 0.00 & j0.023619 & j0.023619 & j0.008939 \\
\hline 5 & 42.16 & 42.16 & 0.00 & j0.029474 & j0.029474 & j0.015758 \\
\hline
\end{tabular}

Table 5.9: Comparison of MATLAB simulation results against published results using PowerWorld Simulator [13]. 


\subsection{Test Data 2}

The following is a comparison of the MATLAB simulation results against both published results from IEEE Standard 399-1997 [4] as well as published results from an ETAP short-circuit verification and validation company document [23] used to verify and validate ETAP's technical accuracy by comparing results with comprehensive test cases, including the example from IEEE Standard 399-1997 [4]. The fault analysis is performed on a 44-bus industrial system whose single-line diagram is shown on the next page in Figure 5.3. The system is a composed of circuits of several voltage levels, local generation, a utility interconnection, and a variety of rotating loads typical of an industrial system operating near to full capacity. The system contains both synchronous and induction motors. The utility is operating at $69 \mathrm{kV}$ and the local generators at $13.8 \mathrm{kV}$. Input data are given on pages 93-95 in Tables 5.10, 5.11, and 5.12. The input file to the MATLAB program and the corresponding MATLAB command window output are provided in the appendix of this report. The fault is a symmetrical three-phase fault at Bus 19. The comparison between the MATLAB simulation results and those published from IEEE Standard 399-1997 [4] and the ETAP shortcircuit verification and validation company document [23] are shown on page 96 in Table 5.13. Note that the results are identical with zero percent error. 


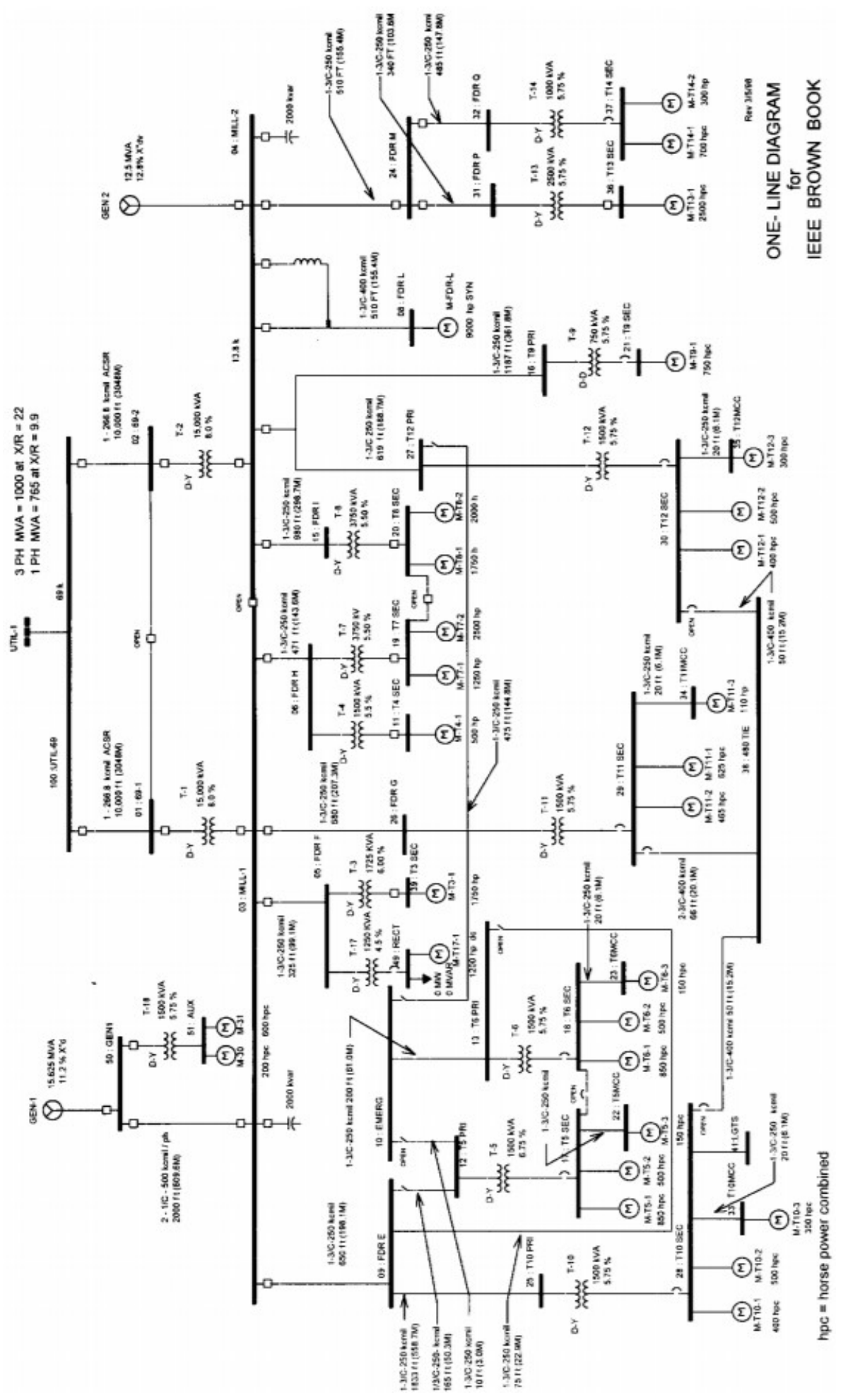

Figure 5.3: Single-line diagram for test data 2 [4]. 


\begin{tabular}{|c|c|c|c|c|c|c|c|c|}
\hline \multirow{2}{*}{$\begin{array}{l}\text { From } \\
\text { bus \# }\end{array}$} & \multirow{2}{*}{$\begin{array}{c}\text { To } \\
\text { bus \# }\end{array}$} & \multirow{2}{*}{ Crkt \# } & \multicolumn{3}{|c|}{ Impedances } & \multirow{2}{*}{$\underset{\text { kV }}{\text { From }}$} & \multirow{2}{*}{$\begin{array}{r}\text { To } \\
\text { kV }\end{array}$} & \multirow{2}{*}{ Tap } \\
\hline & & & $\boldsymbol{R}$ (p.u.) & $X$ (p.u.) & $\boldsymbol{R}$ (p.u.) & & & \\
\hline 1 & 3 & 1 & .00313 & .05324 & 0.0 & 69.00 & 13.800 & 1.00 \\
\hline 2 & 4 & 1 & .00313 & .05324 & 0.0 & 69.00 & 13.800 & 1.00 \\
\hline 5 & 39 & 1 & .04314 & .34514 & 0.0 & 13.80 & 4.160 & 1.00 \\
\hline 5 & 49 & 1 & .05918 & .35510 & 0.0 & 13.80 & .480 & 1.00 \\
\hline 6 & 11 & 1 & .05575 & .36240 & 0.0 & 13.80 & 2.400 & 1.00 \\
\hline 6 & 19 & 1 & .01218 & .14616 & 0.0 & 13.80 & 2.400 & 1.00 \\
\hline 12 & 17 & 1 & .06843 & .44477 & 0.0 & 13.80 & .480 & 1.00 \\
\hline 13 & 18 & 1 & .05829 & .37888 & 0.0 & 13.80 & .480 & 1.00 \\
\hline 15 & 20 & 1 & .01218 & .14616 & 0.0 & 13.80 & 2.400 & 1.00 \\
\hline 16 & 21 & 1 & .15036 & .75178 & 0.0 & 13.80 & .480 & 1.00 \\
\hline 25 & 28 & 1 & .05829 & .37888 & 0.0 & 13.80 & .480 & 1.00 \\
\hline 26 & 29 & 1 & .05829 & .37888 & 0.0 & 13.80 & .480 & 1.00 \\
\hline 27 & 30 & 1 & .05829 & .37888 & 0.0 & 13.80 & .480 & 1.00 \\
\hline 31 & 36 & 1 & .02289 & .22886 & 0.0 & 13.80 & 2.400 & 1.00 \\
\hline 32 & 37 & 1 & .10286 & .56573 & 0.0 & 13.80 & .480 & 1.00 \\
\hline 50 & 51 & 1 & .06395 & .37796 & 0.0 & 13.80 & .480 & 1.00 \\
\hline 3 & 5 & 1 & .00075 & .00063 & 0.0 & & & \\
\hline 3 & 6 & 1 & .00109 & .00091 & 0.0 & & & \\
\hline 3 & 9 & 1 & .00150 & .00125 & 0.0 & & & \\
\hline 3 & 26 & 1 & .00157 & .00131 & 0.0 & & & \\
\hline 4 & 8 & 1 & .00076 & .00092 & 0.0 & & & \\
\hline 4 & 15 & 1 & .00227 & .00189 & 0.0 & & & \\
\hline 4 & 24 & 1 & .00118 & .00098 & 0.0 & & & \\
\hline 4 & 16 & 1 & .00274 & .00229 & 0.0 & & & \\
\hline 4 & 27 & 1 & .00143 & .00119 & 0.0 & & & \\
\hline 9 & 12 & 1 & .00038 & .00032 & 0.0 & & & \\
\hline 9 & 25 & 1 & .00424 & .00353 & 0.0 & & & \\
\hline 10 & 13 & 1 & .00046 & .00039 & 0.0 & & & \\
\hline 10 & 27 & 1 & .00110 & .00091 & 0.0 & & & \\
\hline 17 & 22 & 1 & .03813 & .02451 & 0.0 & & & \\
\hline 18 & 23 & 1 & .03813 & .02451 & 0.0 & & & \\
\hline
\end{tabular}

Table 5.10: Positive-sequence system branch data (p.u., 10MVA) [4]. 


\begin{tabular}{|c|c|c|c|c|c|c|c|c|}
\hline \multirow{2}{*}{$\begin{array}{c}\text { From } \\
\text { bus \# }\end{array}$} & \multirow{2}{*}{$\begin{array}{c}\text { To } \\
\text { bus \# }\end{array}$} & \multirow{2}{*}{ Crkt \# } & \multicolumn{3}{|c|}{ Impedances } & \multirow{2}{*}{$\begin{array}{c}\text { From } \\
\text { kV }\end{array}$} & $\begin{array}{c}\text { To } \\
\text { kV }\end{array}$ & Tap \\
\cline { 4 - 7 } & & & $\boldsymbol{R}$ (p.u.) & X(p.u.) & $\boldsymbol{R}$ (p.u.) & & & \\
\hline 24 & 31 & 1 & .00079 & .00065 & 0.0 & & & \\
\hline 24 & 32 & 1 & .00112 & .00093 & 0.0 & & & \\
\hline 28 & 33 & 1 & .03813 & .02451 & 0.0 & & & \\
\hline 28 & 41 & 1 & .03429 & .02105 & 0.0 & & & \\
\hline 29 & 34 & 1 & .03813 & .02451 & 0.0 & & & \\
\hline 29 & 38 & 1 & .08024 & .07732 & 0.0 & & & \\
\hline 29 & 38 & 2 & .08024 & .07732 & 0.0 & & & \\
\hline 30 & 35 & 1 & .03813 & .02451 & 0.0 & & & \\
\hline 50 & 3 & 1 & .00243 & .00485 & 0.0 & & & \\
\hline 50 & 3 & 2 & .00243 & .00485 & 0.0 & & & \\
\hline 100 & 1 & 1 & .00139 & .00296 & 0.0 & & & \\
\hline 100 & 2 & 1 & .00139 & .00296 & 0.0 & & & \\
\hline
\end{tabular}

Table 5.10: Positive-sequence system branch data (p.u., 10MVA) (Continued) [4].

\begin{tabular}{|c|c|c|c|c|c|}
\hline \multirow{2}{*}{$\begin{array}{c}\text { Generator } \\
\text { bus \# }\end{array}$} & \multirow{2}{*}{ Bus kV } & \multicolumn{4}{|c|}{ Generator impedances } \\
\cline { 3 - 6 } & & $\boldsymbol{R}^{\prime}{ }_{\mathbf{d}}$ & $\boldsymbol{X}_{\mathbf{d}}^{\prime}$ & $\boldsymbol{R}^{\prime \prime}{ }_{\mathbf{d}}$ & $\boldsymbol{X}^{\prime \prime}{ }_{\mathbf{d}}$ \\
\hline 4 & 13.80 & 0.003 & 0.102 & 0.003 & 0.102 \\
\hline 50 & 13.80 & 0.002 & 0.072 & 0.002 & 0.072 \\
\hline 100 & 69.00 & 0.000 & 0.010 & 0.000 & 0.010 \\
\hline
\end{tabular}

Table 5.11: Generator impedances for momentary and interrupting duty (p.u., 10MVA) [4]. 


\begin{tabular}{|c|c|c|c|c|c|c|c|c|}
\hline \multirow{2}{*}{$\begin{array}{l}\text { Motor } \\
\text { bus \# }\end{array}$} & \multirow{2}{*}{ Bus kV } & \multicolumn{2}{|c|}{ Motor } & \multirow{2}{*}{$\begin{array}{c}\text { Motor } \\
\text { MVA }\end{array}$} & \multicolumn{4}{|c|}{ Motor impedances } \\
\hline & & $\#$ & Type & & $\boldsymbol{R}_{\text {mom }}$ & $X_{\text {mom }}$ & $\boldsymbol{R}_{\text {inter }}$ & $X_{\text {inter }}$ \\
\hline 11 & 2.40 & 1 & IM & 0.4750 & 0.352 & 4.219 & 0.879 & 10.547 \\
\hline 17 & 0.48 & 1 & IM & 0.8242 & 0.338 & 3.384 & & \\
\hline 17 & 0.48 & 1 & IM & 0.5000 & 0.802 & 4.008 & 2.004 & 10.020 \\
\hline 18 & 0.48 & 1 & IM & 0.8242 & 0.338 & 3.384 & & \\
\hline 18 & 0.48 & 1 & IM & 0.5000 & 0.802 & 4.008 & 2.004 & 10.020 \\
\hline 19 & 2.40 & 1 & IM & 1.1250 & 0.057 & 1.484 & 0.085 & 2.227 \\
\hline 19 & 2.40 & 1 & IM & 2.3750 & 0.047 & 0.703 & 0.070 & 1.055 \\
\hline 20 & 2.40 & 1 & IM & 1.6625 & 0.067 & 1.005 & 0.100 & 1.507 \\
\hline 20 & 2.40 & 1 & IM & 1.8000 & 0.060 & 1.556 & 0.090 & 2.333 \\
\hline 21 & 0.48 & 1 & IM & 0.7273 & 0.320 & 3.835 & & \\
\hline 22 & 0.48 & 1 & IM & 0.1425 & 1.398 & 19.571 & & \\
\hline 23 & 0.48 & 1 & IM & 0.1425 & 1.398 & 19.571 & & \\
\hline 28 & 0.48 & 1 & IM & 0.5000 & 0.697 & 6.972 & & \\
\hline 28 & 0.48 & 1 & IM & 0.4000 & 0.802 & 4.008 & 2.004 & 10.020 \\
\hline 29 & 0.48 & 1 & IM & 0.6250 & 0.321 & 3.206 & 0.802 & 8.016 \\
\hline 29 & 0.48 & 1 & IM & 0.4650 & 1.199 & 5.998 & & \\
\hline 30 & 0.48 & 1 & IM & 0.3879 & 0.431 & 5.166 & 1.077 & 12.916 \\
\hline 30 & 0.48 & 1 & IM & 0.5000 & 1.116 & 5.578 & & \\
\hline 33 & 0.48 & 1 & IM & 0.2875 & 0.809 & 9.701 & & \\
\hline 34 & 0.48 & 1 & IM & 0.1100 & 3.621 & 25.354 & & \\
\hline 35 & 0.48 & 1 & IM & 0.2875 & 0.809 & 9.701 & & \\
\hline 36 & 2.40 & 1 & IM & 2.2500 & 0.025 & 0.818 & 0.062 & 2.045 \\
\hline 37 & 0.48 & 1 & IM & 0.6788 & 0.246 & 2.952 & 0.615 & 7.381 \\
\hline 37 & 0.48 & 1 & IM & 0.3000 & 1.859 & 9.296 & & \\
\hline 39 & 4.16 & 1 & IM & 1.6625 & 0.034 & 1.005 & 0.051 & 1.507 \\
\hline 49 & 0.48 & 1 & IM & 1.2500 & 0.264 & 2.640 & & \\
\hline 51 & 0.48 & 1 & IM & 0.2000 & 1.432 & 10.020 & 3.579 & 25.050 \\
\hline 51 & 0.48 & 1 & IM & 0.5700 & 0.408 & 4.893 & & \\
\hline 8 & 13.80 & 1 & SM & 9.0000 & 0.006 & 0.222 & 0.010 & 0.333 \\
\hline
\end{tabular}

Table 5.12: Motor impedances for momentary and interrupting duty (p.u., 10MVA) [4]. 


\begin{tabular}{|l|c|c|c|c|}
\hline Fault at Bus 19 & $\begin{array}{c}\text { IEEE Std. } \\
399-1997 \\
\text { Example }\end{array}$ & ETAP & MATLAB & \% Error* \\
\hline Prefault Voltage (kV) & 2.40 & 2.40 & 2.40 & 0.00 \\
\hline Fault current (p.u., 10MVA) & 7.67 & - & 7.67 & 0.00 \\
\hline Fault current (kA) & 18.449 & 18.453 & 18.449 & 0.00 \\
\hline Voltage-to-ground during fault (p.u.) & 0.00 & 0.00 & 0.00 & 0.00 \\
\hline Fault contribution from Bus 6 (p.u.) & 5.57 & - & 5.57 & 0.00 \\
\hline Fault contribution from Bus 6 (kA) & 13.418 & 13.422 & 13.418 & 0.00 \\
\hline Voltage-to-ground at Bus 6 (p.u.) & 0.82 & 0.82 & 0.82 & 0.00 \\
\hline Fault contribution from motors (p.u.) & 2.09 & - & 2.09 & 0.00 \\
\hline Fault contribution from motors (kA) & 5.03 & 5.03 & 5.03 & 0.00 \\
\hline
\end{tabular}

Table 5.13: Comparison of MATLAB simulation results against published results from IEEE Std. 399-1997 [4] and an ETAP short-circuit verification and validation company document [23].

*Percent Error is between the MATLAB simulation results and those published from IEEE Standard 399-1997 [4]. 


\subsection{Test Data 3}

The MATLAB fault current analysis program will now be used to perform fault analysis of a power system with converter-based distributed energy resources to demonstrate how to model the fault current contributions to the power system within the framework of conventional fault analysis techniques. The fault analysis is performed on the same system as in the previous set of test data from IEEE Standard 399-1997 [4], except that the 12.5 MVA local synchronous generator identified by "GEN 2" on the single-line diagram shown earlier in Figure 5.3 is replaced with an equivalent 12.5 MVA series converter-connected generator with a grid-side inverter (e.g., solar PV and wind turbine generators with energy storage) connected through a transformer to the distribution system voltage level $(13.8 \mathrm{kV})$ as shown on page 100 in Figure 5.4. As in the previous set of test data from IEEE Standard 399-1997 [4], the system base apparent power is 10 MVA. The input file to the MATLAB program and the corresponding MATLAB command window output are provided in the appendix of this report. The fault is a symmetrical three-phase fault at Bus 4, i.e., the inverter transformer output terminals. The MATLAB simulation results are shown on page 101 in Table 5.14.

The rated output current of the inverter on the high-voltage side of the transformer can be calculated from

$$
\left|\mathbf{I}_{\text {inv(rated) }}\right|=\frac{12.5 \mathrm{MVA}}{\sqrt{3} \times 13.8 \mathrm{kV}}=523 \mathrm{~A}
$$

As mentioned in Chapter 4, series converter-connected generators with a grid-side inverter effectively isolate the electrical generator and its specific electrical performance characteristics from the power system in the event of a fault on the host system and can provide a controlled fault current response during the entire fault duration [5]. Therefore, utilities typically require a larger fault current contribution from such generators to assist power system protection in the detection of fault currents. As a result, modern grid-side inverter controls are typically designed to supply a constant value of three-phase fault current that is related to the rated current by

$$
\left|\mathbf{I}_{\text {inv(sc) }}\right|=\alpha\left|\mathbf{I}_{\text {inv(rated) }}\right|
$$

where $\alpha \leq 3$ for most current inverter designs [5] - [12]. For this set of test data, we will use $\alpha=2$. In this case, the correct inverter fault current contribution is given by

$$
\left|\mathbf{I}_{\text {inv(sc) }}\right|=2 \times 523 \mathrm{~A}=1,046 \mathrm{~A}
$$

In per unit, 


$$
\left|\mathbf{I}_{\text {inv(sc) }}\right|=\frac{1,046 \mathrm{~A}}{I_{\text {base }}}=\frac{1,046 \mathrm{~A}}{\frac{S_{\text {base }}}{\sqrt{3} \times V_{\text {LL,base }}}}=\frac{1,046 \mathrm{~A}}{\frac{10 \mathrm{MVA}}{\sqrt{3} \times 13.8 \mathrm{kV}}}=\frac{1,046 \mathrm{~A}}{418.4 \mathrm{~A}}=2.5 \mathrm{pu}
$$

To overcome the difficulty of integrating a constant current source into the framework of conventional fault analysis, it must be replaced by a voltage source behind an inductive reactance (as in the case of synchronous generators) using a Norton-Thevenin source transformation such that the conventional fault current calculation techniques described earlier in Chapters 2 and 3 may be employed. However, it is generally incorrect to assume that a series converter-connected generator with a grid-side inverter can be modeled by an internal generated voltage $\mathbf{E}_{A}$ behind a positive-sequence impedance $Z_{1}$ such that $\left|\mathbf{I}_{\text {inv(sc) }}\right|=\left|\mathbf{E}_{A}\right| /\left|Z_{1}\right|$. This representation will produce the correct inverter fault current contribution for one fault location only, namely at the inverter transformer output terminals. At other fault locations on the power system, the source transformation will produce a lower and incorrect inverter fault current contribution than that given by Equation (5.1). Since the equivalent positive-sequence impedance varies with the inverter transformer terminal voltage which is a function of the fault current, the equivalent positive-sequence impedance must be determined by an iterative process to obtain the correct inverter fault current contribution at all other fault locations on the power system (as will be seen in the next set of test data). Therefore, the inverter fault current contribution can be calculated from

$$
\left|\mathbf{I}_{\text {inv(sc) }}\right|=\frac{\left|\mathbf{E}_{A}\right|-\left|\mathbf{V}_{A 1}\right|}{\left|Z_{1}\right|}
$$

where $\mathbf{V}_{A 1}$ is the positive-sequence terminal voltage of the inverter transformer. For a fault at the inverter transformer output terminals, i.e., $\mathbf{V}_{A 1}=0$ per unit, this simplifies to

$$
\left|\mathbf{I}_{\text {inv(sc) }}\right|=\frac{\left|\mathbf{E}_{A}\right|}{\left|Z_{1}\right|}
$$

To obtain the desired inverter fault current contribution, the equivalent positive-sequence impedance can be calculated according to Equation (5.3)

$$
\left|Z_{1}\right|=\frac{\left|\mathbf{E}_{A}\right|}{\left|\mathbf{I}_{\mathrm{inv}(\mathrm{sc})}\right|}=\frac{1.0 \mathrm{pu}}{2.5 \mathrm{pu}}=0.4 \mathrm{pu}
$$

But 


$$
\left|Z_{1}\right|=\sqrt{R_{1}^{2}+X_{1}^{2}}=\sqrt{R_{\mathrm{t}}^{2}+\left(X_{\mathrm{t}}+X_{\mathrm{inv}}\right)^{2}}=0.4 \mathrm{pu}
$$

which leads to

$$
\sqrt{(0.00313 \mathrm{pu})^{2}+\left(0.05324 \mathrm{pu}+X_{\mathrm{inv}}\right)^{2}} \approx 0.05324 \mathrm{pu}+X_{\mathrm{inv}}=0.4 \mathrm{pu}
$$

Hence, to obtain the desired inverter fault current contribution, the series converter-connected generator with a grid-side inverter (including the inverter transformer) can be modelled by an equivalent voltage source behind a subtransient reactance of 0.4 per unit. From the MATLAB simulation results shown on page 101 in Table 5.14, the inverter fault current contribution from Bus 52 to the symmetrical three-phase fault at Bus 4, i.e., the inverter transformer output terminals, is the calculated desired value of 2.5 per unit or $1,046 \mathrm{~A}$ as expected. This result verifies and validates the MATLAB model of the series converterconnected generator with a grid-side inverter, thereby ensuring the technical accuracy of the model within the framework of conventional fault analysis techniques. 


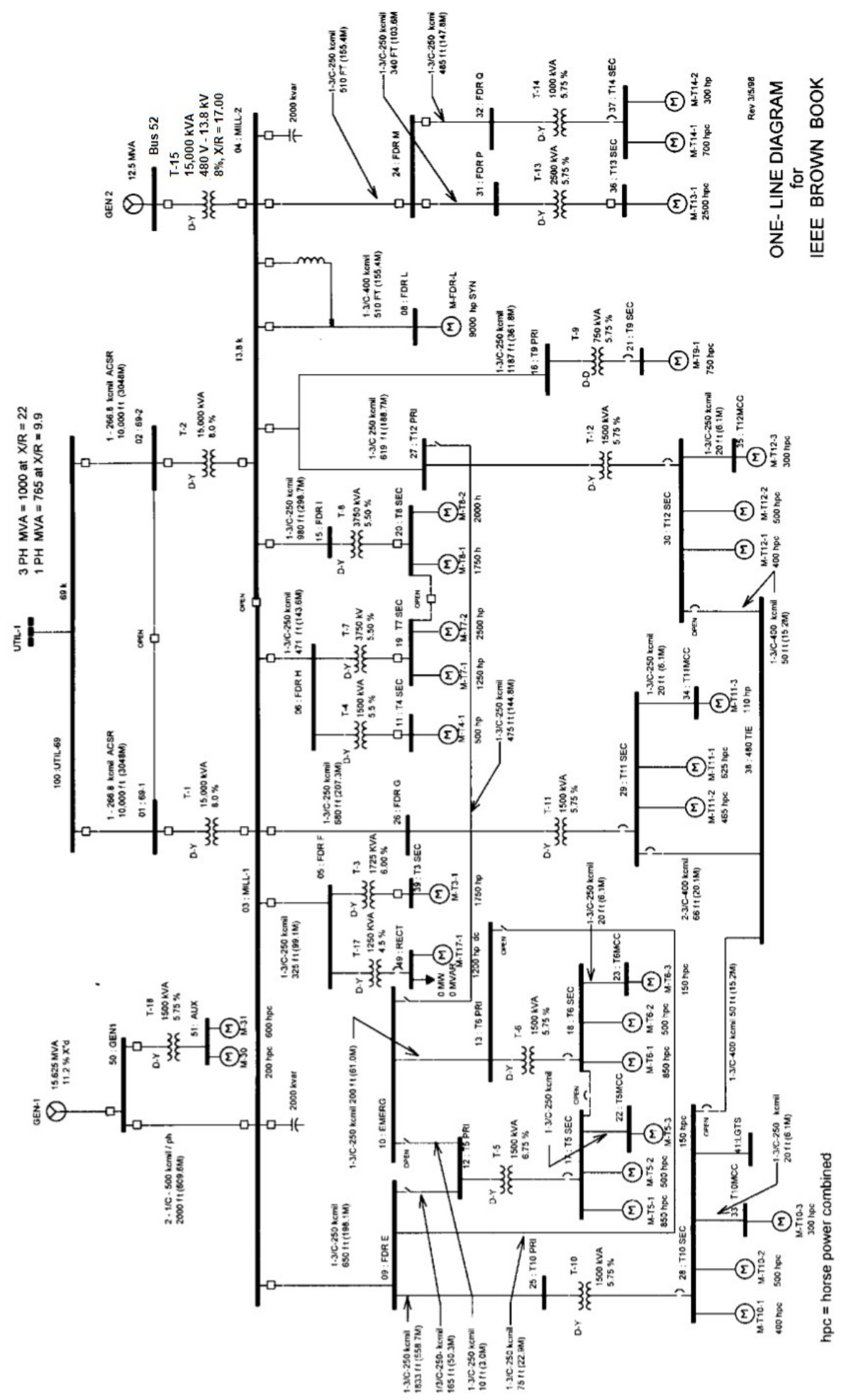

Figure 5.4: Single-line diagram for test data 3 [4]. 


\begin{tabular}{|c|c|c|c|}
\hline Fault at Bus 4 & MATLAB & $\begin{array}{c}\text { Correct } \\
\text { Calculated } \\
\text { Value }\end{array}$ & $\%$ Error \\
\hline Prefault Voltage (kV) & 13.8 & - & - \\
\hline Fault current (p.u., 10MVA) & 25.944 & - & - \\
\hline Fault current $(\mathrm{kA})$ & 10.855 & - & - \\
\hline Voltage-to-ground during fault (p.u.) & 0.000 & - & - \\
\hline Fault contribution from Bus 2 (p.u.) & 15.257 & - & - \\
\hline Fault contribution from Bus $2(\mathrm{kA})$ & 6.384 & - & - \\
\hline Voltage-to-ground at Bus 2 (p.u.) & 0.814 & - & - \\
\hline Fault contribution from Bus 8 (p.u.) & 4.484 & - & - \\
\hline Fault contribution from Bus 8 (kA) & 1.876 & - & - \\
\hline Voltage-to-ground at Bus 8 (p.u.) & 0.005 & - & - \\
\hline Fault contribution from Bus 15 (p.u.) & 1.315 & - & - \\
\hline Fault contribution from Bus $15(\mathrm{kA})$ & 0.550 & - & - \\
\hline Voltage-to-ground at Bus 15 (p.u.) & 0.004 & - & - \\
\hline Fault contribution from Bus 16 (p.u.) & 0.217 & - & - \\
\hline Fault contribution from Bus $16(\mathrm{kA})$ & 0.091 & - & - \\
\hline Voltage-to-ground at Bus 16 (p.u.) & 0.001 & - & - \\
\hline Fault contribution from Bus 24 (p.u.) & 1.304 & - & - \\
\hline Fault contribution from Bus $24(\mathrm{kA})$ & 0.546 & - & - \\
\hline Voltage-to-ground at Bus 24 (p.u.) & 0.002 & - & - \\
\hline Fault contribution from Bus 27 (p.u.) & 0.878 & - & - \\
\hline Fault contribution from Bus $27(\mathrm{kA})$ & 0.367 & - & - \\
\hline Voltage-to-ground at Bus 27 (p.u.) & 0.002 & - & - \\
\hline Fault contribution from Bus 52 (p.u.) & 2.500 & 2.500 & 0.00 \\
\hline Fault contribution from Bus $52(\mathrm{kA})$ & 1.046 & 1.046 & 0.00 \\
\hline Voltage-to-ground at Bus 52 (р.u.) & 0.133 & - & - \\
\hline
\end{tabular}

Table 5.14: Comparison of MATLAB simulation results against calculated inverter fault current contribution.

For comparison, the fault analysis performed above for a symmetrical three-phase fault at Bus 4 is repeated on the same system used in the previous set of test data from IEEE Standard 399-1997 [4], with the original 12.5 MVA local synchronous generator identified by "GEN 2" on the single-line diagram shown earlier in Figure 5.3. The input file to the MATLAB program and the corresponding command window output are provided in the appendix of this report. The MATLAB simulation results are shown on the next page in Table 
5.15. From the MATLAB simulation results shown in Table 5.15, it is evident that the fault current contribution during the symmetrical three-phase fault at Bus 4 from the original 12.5 MVA local synchronous generator identified by "GEN 2" on the single-line diagram shown earlier in Figure 5.3 is significantly greater than the fault current contribution from the 12.5 MVA series converter-connected generator with a grid-side inverter identified by "GEN 2" on the single-line diagram shown earlier in Figure 5.4.

\begin{tabular}{|l|c|c|}
\hline Fault at Bus 4 & $\begin{array}{c}12.5 \text { MVA } \\
\text { Synchronous } \\
\text { Generator }\end{array}$ & $\begin{array}{c}12.5 \text { MVA } \\
\text { Grid-side } \\
\text { Inverter }\end{array}$ \\
\hline Prefault Voltage (kV) & 13.8 & 13.8 \\
\hline Fault current (p.u., 10MVA) & 33.244 & 25.944 \\
\hline Fault current (kA) & 13.909 & 10.855 \\
\hline Voltage-to-ground during fault (p.u.) & 0.000 & 0.000 \\
\hline Fault contribution from Bus 2 (p.u.) & 15.257 & 15.257 \\
\hline Fault contribution from Bus 2 (kA) & 6.384 & 6.384 \\
\hline Voltage-to-ground at Bus 2 (p.u.) & 0.814 & 0.814 \\
\hline Fault contribution from Bus 8 (p.u.) & 4.484 & 4.484 \\
\hline Fault contribution from Bus 8 (kA) & 1.876 & 1.876 \\
\hline Voltage-to-ground at Bus 8 (p.u.) & 0.005 & 0.005 \\
\hline Fault contribution from Bus 15 (p.u.) & 1.315 & 1.315 \\
\hline Fault contribution from Bus 15 (kA) & 0.550 & 0.550 \\
\hline Voltage-to-ground at Bus 15 (p.u.) & 0.004 & 0.004 \\
\hline Fault contribution from Bus 16 (p.u.) & 0.217 & 0.217 \\
\hline Fault contribution from Bus 16 (kA) & 0.091 & 0.091 \\
\hline Voltage-to-ground at Bus 16 (p.u.) & 0.001 & 0.001 \\
\hline Fault contribution from Bus 24 (p.u.) & 1.304 & 1.304 \\
\hline Fault contribution from Bus 24 (kA) & 0.546 & 0.546 \\
\hline Voltage-to-ground at Bus 24 (p.u.) & 0.002 & 0.002 \\
\hline Fault contribution from Bus 27 (p.u.) & 0.878 & 0.878 \\
\hline Fault contribution from Bus 27 (kA) & 0.367 & 0.002 \\
\hline Voltage-to-ground at Bus 27 (p.u.) & 0.002 & 9.500 \\
\hline Fault contribution from GEN 2 (p.u.) & 9.789 & 4.096 \\
\hline Fault contribution from GEN 2 (kA) & & \\
\hline
\end{tabular}

Table 5.15: Comparison of fault current contributions from a 12.5 MVA synchronous generator and a 12.5 MVA series converter-connected generator with a grid-side inverter. 


\subsection{Test Data 4}

The MATLAB fault current analysis program will again be used to perform fault analysis of a power system with converter-based distributed energy resources, except that the location of the fault on the power system will be other than the inverter transformer output terminals to demonstrate the iterative process used to obtain the correct inverter fault current contribution at all other fault locations on the power system. The fault analysis is performed on the same system used in the previous set of test data shown earlier in Figure 5.4. The input file to the MATLAB program and the corresponding MATLAB command window output are provided in the appendix of this report. The fault is a symmetrical three-phase fault at Bus 20. The MATLAB simulation results are shown on pages 109 and 110 in Tables 5.16 and 5.17, respectively.

The rated output current of the inverter on the high-voltage side of the transformer can be calculated from

$$
\left|\mathbf{I}_{\text {inv(rated) }}\right|=\frac{12.5 \mathrm{MVA}}{\sqrt{3} \times 13.8 \mathrm{kV}}=523 \mathrm{~A}
$$

As mentioned earlier, series converter-connected generators with a grid-side inverter effectively isolate the electrical generator and its specific electrical performance characteristics from the power system in the event of a fault on the host system and can provide a controlled fault current response during the entire fault duration [5]. Therefore, utilities typically require a larger fault current contribution from such generators to assist power system protection in the detection of fault currents. As a result, modern grid-side inverter controls are typically designed to supply a constant value of three-phase fault current that is related to the rated current by Equation (5.1), where $\alpha \leq 3$ for most current inverter designs [5] - [12]. For this set of test data, we will use $\alpha=2$. In this case, the correct inverter fault current contribution is given by

$$
\left|\mathbf{I}_{\text {inv(sc) }}\right|=2 \times 523 \mathrm{~A}=1,046 \mathrm{~A}
$$

In per unit,

$$
\left|\mathbf{I}_{\text {inv(sc) }}\right|=\frac{1,046 \mathrm{~A}}{I_{\text {base }}}=\frac{1,046 \mathrm{~A}}{\frac{S_{\text {base }}}{\sqrt{3} \times V_{\text {LL,base }}}}=\frac{1,046 \mathrm{~A}}{\frac{10 \mathrm{MVA}}{\sqrt{3} \times 13.8 \mathrm{kV}}}=\frac{1,046 \mathrm{~A}}{418.4 \mathrm{~A}}=2.5 \mathrm{pu}
$$

To overcome the difficulty of integrating a constant current source into the framework of conventional fault analysis, it must be replaced by a voltage source behind an inductive reactance (as in the case of synchronous generators) using a Norton-Thevenin source transformation such that the conventional fault current calculation techniques described earlier in Chapters 2 and 3 may be employed. However, it is 
generally incorrect to assume that a series converter-connected generator with a grid-side inverter can be modeled by an internal generated voltage $\mathbf{E}_{A}$ behind a positive-sequence impedance $Z_{1}$ such that $\left|\mathbf{I}_{\text {inv(sc) }}\right|=\left|\mathbf{E}_{A}\right| /\left|Z_{1}\right|$. This representation will produce the correct inverter fault current contribution for one fault location only, namely at the inverter transformer output terminals. At other fault locations on the power system, the source transformation will produce a lower and incorrect inverter fault current contribution than that given by Equation (5.1). Since the equivalent positive-sequence impedance varies with the inverter transformer terminal voltage which is a function of the fault current, the equivalent positive-sequence impedance must be determined by an iterative process to obtain the correct inverter fault current contribution at all other fault locations on the power system. Therefore, the inverter fault current contribution can be calculated from Equation (5.2), where $\mathbf{V}_{A 1}$ is the positive-sequence terminal voltage of the inverter transformer. For a fault at the inverter transformer output terminals, i.e., $\mathbf{V}_{A 1}=0$ per unit, this simplifies to Equation (5.3) as seen in the previous set of test data. However, at all other fault locations on the power system, Equation (5.3) will produce a lower and incorrect inverter fault current contribution than that given by Equation (5.1). To obtain the desired inverter fault current contribution at all other fault locations on the power system, the equivalent positive-sequence impedance must be determined by an iterative process until it produces an inverter fault current contribution that converges to the desired value.

For the first iteration, we will assume $\mathbf{V}_{A 1}=0$ per unit as an initial estimated value for the inverter transformer terminal voltage during the fault on the power system. This value will then be used to calculate the equivalent positive-sequence impedance according to Equation (5.2), which will be supplied as input data to the MATLAB program. The output of the MATLAB program will be observed to determine if the desired value of the inverter fault current contribution matches the MATLAB simulation results based on the estimated values of the inverter transformer terminal voltage and the equivalent positive-sequence impedance. If any mismatch exists, subsequent iterations are performed until the desired value of the inverter fault current contribution matches the MATLAB simulation results. During each subsequent iteration, the starting value for the inverter transformer terminal voltage during the fault on the power system will be updated based on the MATLAB simulation results of the previous iteration and the process described above will be repeated until the corresponding equivalent positive-sequence impedance calculated from Equation (5.2) produces the desired inverter fault current contribution. Substituting $\mathbf{V}_{A 1}=0$ per unit into Equation (5.2) yields

$$
\left|Z_{1}\right|=\frac{\left|\mathbf{E}_{A}\right|}{\left|\mathbf{I}_{\mathrm{inv}(\mathrm{sc})}\right|}=\frac{1.0 \mathrm{pu}}{2.5 \mathrm{pu}}=0.4 \mathrm{pu}
$$

But 


$$
\left|Z_{1}\right|=\sqrt{R_{1}^{2}+X_{1}^{2}}=\sqrt{R_{\mathrm{t}}^{2}+\left(X_{\mathrm{t}}+X_{\mathrm{inv}}\right)^{2}}=0.4 \mathrm{pu}
$$

which leads to

$$
\sqrt{(0.00313 \mathrm{pu})^{2}+\left(0.05324 \mathrm{pu}+X_{\mathrm{inv}}\right)^{2}} \approx 0.05324 \mathrm{pu}+X_{\mathrm{inv}}=0.4 \mathrm{pu}
$$

From the MATLAB simulation results shown on page 109 in Table 5.16, the inverter fault current contribution during the symmetrical three-phase fault at Bus 20 is 0.536 per unit or $224 \mathrm{~A}$. As a result, the equivalent positive-sequence impedance must be further adjusted by recalculating Equation (5.2) until it produces an inverter fault current contribution that converges to the desired value.

For the second iteration, we will use $\left|\mathbf{E}_{A}\right|-\left|\mathbf{V}_{A 1}\right|=0.21440$ per unit as a starting value based on the MATLAB simulation results from the previous iteration, i.e.,

$$
\left|\mathbf{E}_{A}\right|-\left|\mathbf{V}_{A 1}\right|=\left|\mathbf{I}_{\text {inv(sc) }}\right| \times\left|Z_{1}\right|=0.536 \mathrm{pu} \times 0.4 \mathrm{pu}=0.21440 \mathrm{pu}
$$

Repeating the process described above with this updated value yields

$$
\left|Z_{1}\right|=\frac{\left|\mathbf{E}_{A}\right|-\left|\mathbf{V}_{A 1}\right|}{\left|\mathbf{I}_{\text {inv(sc) }}\right|}=\frac{0.21440 \mathrm{pu}}{2.5 \mathrm{pu}}=0.08576 \mathrm{pu}
$$

which leads to

$$
\left|Z_{1}\right|=\sqrt{R_{1}^{2}+X_{1}^{2}} \approx X_{1}=X_{\mathrm{t}}+X_{\mathrm{inv}}=0.05324 \mathrm{pu}+X_{\mathrm{inv}}=0.08576 \mathrm{pu}
$$

From the MATLAB simulation results shown on page 109 in Table 5.16, the inverter fault current contribution during the symmetrical three-phase fault at Bus 20 is 1.934 per unit or $809 \mathrm{~A}$. As a result, the equivalent positive-sequence impedance must be further adjusted by recalculating Equation (5.2) until it produces an inverter fault current contribution that converges to the desired value.

For the third iteration, we will use $\left|\mathbf{E}_{A}\right|-\left|\mathbf{V}_{A 1}\right|=0.16586$ per unit as a starting value based on the MATLAB simulation results from the previous iteration, i.e.,

$$
\left|\mathbf{E}_{A}\right|-\left|\mathbf{V}_{A 1}\right|=\left|\mathbf{I}_{\text {inv }(\mathrm{sc})}\right| \times\left|Z_{1}\right|=1.934 \mathrm{pu} \times 0.08576 \mathrm{pu}=0.16586 \mathrm{pu}
$$


Repeating the process described above with this updated value yields

$$
\left|Z_{1}\right|=\frac{\left|\mathbf{E}_{A}\right|-\left|\mathbf{V}_{A 1}\right|}{\left|\mathbf{I}_{\text {inv(sc) }}\right|}=\frac{0.16586 \mathrm{pu}}{2.5 \mathrm{pu}}=0.06634 \mathrm{pu}
$$

which leads to

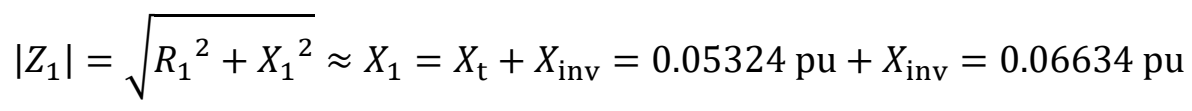

From the MATLAB simulation results shown on page 109 in Table 5.16, the inverter fault current contribution during the symmetrical three-phase fault at Bus 20 is 2.305 per unit or $964 \mathrm{~A}$. As a result, the equivalent positive-sequence impedance must be further adjusted by recalculating Equation (5.2) until it produces an inverter fault current contribution that converges to the desired value.

For the fourth iteration, we will use $\left|\mathbf{E}_{A}\right|-\left|\mathbf{V}_{A 1}\right|=0.15291$ per unit as a starting value based on the MATLAB simulation results from the previous iteration, i.e.,

$$
\left|\mathbf{E}_{A}\right|-\left|\mathbf{V}_{A 1}\right|=\left|\mathbf{I}_{\text {inv(sc) }}\right| \times\left|Z_{1}\right|=2.305 \mathrm{pu} \times 0.06634 \mathrm{pu}=0.15291 \mathrm{pu}
$$

Repeating the process described above with this updated value yields

$$
\left|Z_{1}\right|=\frac{\left|\mathbf{E}_{A}\right|-\left|\mathbf{V}_{A 1}\right|}{\left|\mathbf{I}_{\text {inv(sc) }}\right|}=\frac{0.15291 \mathrm{pu}}{2.5 \mathrm{pu}}=0.06117 \mathrm{pu}
$$

which leads to

$$
\left|Z_{1}\right|=\sqrt{{R_{1}}^{2}+X_{1}^{2}} \approx X_{1}=X_{\mathrm{t}}+X_{\mathrm{inv}}=0.05324 \mathrm{pu}+X_{\mathrm{inv}}=0.06117 \mathrm{pu}
$$

From the MATLAB simulation results shown on page 109 in Table 5.16, the inverter fault current contribution during the symmetrical three-phase fault at Bus 20 is 2.430 per unit or 1,017 A. As a result, the equivalent positive-sequence impedance must be further adjusted by recalculating Equation (5.2) until it produces an inverter fault current contribution that converges to the desired value.

For the fifth iteration, we will use $\left|\mathbf{E}_{A}\right|-\left|\mathbf{V}_{A 1}\right|=0.14864$ per unit as a starting value based on the MATLAB simulation results from the previous iteration, i.e., 


$$
\left|\mathbf{E}_{A}\right|-\left|\mathbf{V}_{A 1}\right|=\left|\mathbf{I}_{\text {inv }(\mathrm{sc})}\right| \times\left|Z_{1}\right|=2.430 \mathrm{pu} \times 0.06117 \mathrm{pu}=0.14864 \mathrm{pu}
$$

Repeating the process described above with this updated value yields

$$
\left|Z_{1}\right|=\frac{\left|\mathbf{E}_{A}\right|-\left|\mathbf{V}_{A 1}\right|}{\left|\mathbf{I}_{\text {inv(sc) }}\right|}=\frac{0.14864 \mathrm{pu}}{2.5 \mathrm{pu}}=0.05946 \mathrm{pu}
$$

which leads to

$$
\left|Z_{1}\right|=\sqrt{R_{1}^{2}+X_{1}^{2}} \approx X_{1}=X_{\mathrm{t}}+X_{\mathrm{inv}}=0.05324 \mathrm{pu}+X_{\mathrm{inv}}=0.05946 \mathrm{pu}
$$

From the MATLAB simulation results shown on page 109 in Table 5.16, the inverter fault current contribution during the symmetrical three-phase fault at Bus 20 is 2.474 per unit or 1,035 A. As a result, the equivalent positive-sequence impedance must be further adjusted by recalculating Equation (5.2) until it produces an inverter fault current contribution that converges to the desired value.

For the sixth iteration, we will use $\left|\mathbf{E}_{A}\right|-\left|\mathbf{V}_{A 1}\right|=0.14710$ per unit as a starting value based on the MATLAB simulation results from the previous iteration, i.e.,

$$
\left|\mathbf{E}_{A}\right|-\left|\mathbf{V}_{A 1}\right|=\left|\mathbf{I}_{\mathrm{inv}(\mathrm{sc})}\right| \times\left|Z_{1}\right|=2.474 \mathrm{pu} \times 0.05946 \mathrm{pu}=0.14710 \mathrm{pu}
$$

Repeating the process described above with this updated value yields

$$
\left|Z_{1}\right|=\frac{\left|\mathbf{E}_{A}\right|-\left|\mathbf{V}_{A 1}\right|}{\left|\mathbf{I}_{\text {inv(sc) }}\right|}=\frac{0.14710 \mathrm{pu}}{2.5 \mathrm{pu}}=0.05884 \mathrm{pu}
$$

which leads to

$$
\left|Z_{1}\right|=\sqrt{{R_{1}}^{2}+X_{1}^{2}} \approx X_{1}=X_{\mathrm{t}}+X_{\mathrm{inv}}=0.05324 \mathrm{pu}+X_{\mathrm{inv}}=0.05884 \mathrm{pu}
$$

From the MATLAB simulation results shown on page 109 in Table 5.16, the inverter fault current contribution during the symmetrical three-phase fault at Bus 20 is 2.490 per unit or 1,042 A. As a result, the equivalent positive-sequence impedance must be further adjusted by recalculating Equation (5.2) until it produces an inverter fault current contribution that converges to the desired value. 
For the seventh iteration, we will use $\left|\mathbf{E}_{A}\right|-\left|\mathbf{V}_{A 1}\right|=0.14651$ per unit as a starting value based on the MATLAB simulation results from the previous iteration, i.e.,

$$
\left|\mathbf{E}_{A}\right|-\left|\mathbf{V}_{A 1}\right|=\left|\mathbf{I}_{\text {inv(sc) }}\right| \times\left|Z_{1}\right|=2.490 \mathrm{pu} \times 0.05884 \mathrm{pu}=0.14651 \mathrm{pu}
$$

Repeating the process described above with this updated value yields

$$
\left|Z_{1}\right|=\frac{\left|\mathbf{E}_{A}\right|-\left|\mathbf{V}_{A 1}\right|}{\left|\mathbf{I}_{\text {inv(sc) }}\right|}=\frac{0.14651 \mathrm{pu}}{2.5 \mathrm{pu}}=0.05860 \mathrm{pu}
$$

which leads to

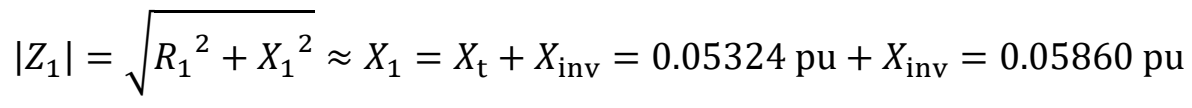

From the MATLAB simulation results shown on the next page in Table 5.16, the inverter fault current contribution during the symmetrical three-phase fault at Bus 20 is 2.495 per unit or 1,044 A. As a result, the equivalent positive-sequence impedance must be further adjusted by recalculating Equation (5.2) until it produces an inverter fault current contribution that converges to the desired value.

For the eighth iteration, we will use $\left|\mathbf{E}_{A}\right|-\left|\mathbf{V}_{A 1}\right|=0.14621$ per unit as a starting value based on the MATLAB simulation results from the previous iteration, i.e.,

$$
\left|\mathbf{E}_{A}\right|-\left|\mathbf{V}_{A 1}\right|=\left|\mathbf{I}_{\text {inv(sc) }}\right| \times\left|Z_{1}\right|=2.495 \mathrm{pu} \times 0.05860 \mathrm{pu}=0.14621 \mathrm{pu}
$$

Repeating the process described above with this updated value yields

$$
\left|Z_{1}\right|=\frac{\left|\mathbf{E}_{A}\right|-\left|\mathbf{V}_{A 1}\right|}{\left|\mathbf{I}_{\text {inv(sc) }}\right|}=\frac{0.14621 \mathrm{pu}}{2.5 \mathrm{pu}}=0.05848 \mathrm{pu}
$$

which leads to

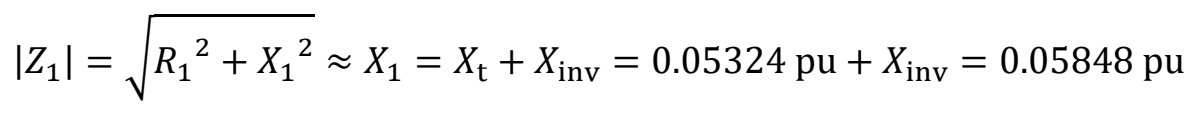

From the MATLAB simulation results shown on the next page in Table 5.16, the inverter fault current contribution during the symmetrical three-phase fault at Bus 20 is the calculated desired value of 2.5 per 
unit or 1,046 $\mathrm{A}$ as expected. As a result, the equivalent positive-sequence impedance need not be further adjusted by recalculating Equation (5.2) due to that the calculated inverter fault current contribution has converged to the desired value and no further iterations are required. Hence, to obtain the desired inverter fault current contribution, the series converter-connected generator with a grid-side inverter (including the inverter transformer) can be modelled by an equivalent voltage source behind a subtransient reactance of 0.05848 per unit. This result verifies and validates the MATLAB model of the series converter-connected generator with a grid-side inverter, thereby ensuring the technical accuracy of the model within the framework of conventional fault analysis techniques.

\begin{tabular}{|l|c|c|c|}
\hline & $\begin{array}{c}\text { Inverter } \\
\text { Fault } \\
\text { Contribution } \\
\text { Fault at Bus 20, 10MVA) }\end{array}$ & $\begin{array}{c}\text { Inverter } \\
\text { Fault } \\
\text { Contribution } \\
\text { (kA) }\end{array}$ & \% Error \\
\hline Iteration 1 & 0.536 & 0.224 & 78.56 \\
\hline Iteration 2 & 1.934 & 0.809 & 22.64 \\
\hline Iteration 3 & 2.305 & 0.964 & 7.80 \\
\hline Iteration 4 & 2.430 & 1.017 & 2.80 \\
\hline Iteration 5 & 2.474 & 1.035 & 1.04 \\
\hline Iteration 6 & 2.490 & 1.042 & 0.40 \\
\hline Iteration 7 & 2.495 & 1.044 & 0.20 \\
\hline Iteration 8 & 2.500 & 1.046 & 0.00 \\
\hline
\end{tabular}

Table 5.16: Comparison of MATLAB simulation results against calculated inverter fault current contribution.

For comparison, the fault analysis performed above for a symmetrical three-phase fault at Bus 20 is repeated on the same system used in test data 2 from IEEE Standard 399-1997 [4], with the original 12.5 MVA local synchronous generator identified by "GEN 2" on the single-line diagram shown earlier in Figure 5.3. The input file to the MATLAB program and the corresponding command window output are provided in the appendix of this report. The MATLAB simulation results are shown on the next page in Table 5.17. From the MATLAB simulation results shown in Table 5.17, it is evident that the fault current contribution during the symmetrical three-phase fault at Bus 20 from the original 12.5 MVA local synchronous generator identified by "GEN 2" on the single-line diagram shown earlier in Figure 5.3 is less than the fault current contribution from the 12.5 MVA series converter-connected generator with a grid-side inverter identified by "GEN 2" on the single-line diagram shown earlier in Figure 5.4. This result emphasizes the need to develop mathematical and software simulation models that approximate the response of converter-based distributed energy resources during a fault on the transmission or distribution system in order to determine the fault current contributions to the electrical grid that a transmission or distribution utility needs to reflect in their 
connection impact assessments essential for the design and application of distribution and protective apparatuses used in these systems.

\begin{tabular}{|l|c|c|}
\hline Fault at Bus 20 & $\begin{array}{c}12.5 \text { MVA } \\
\text { Synchronous } \\
\text { Generator }\end{array}$ & $\begin{array}{c}12.5 \text { MVA } \\
\text { Grid-side } \\
\text { Inverter }\end{array}$ \\
\hline Prefault Voltage (kV) & 2.40 & 2.40 \\
\hline Fault current (p.u., 10MVA) & 7.188 & 7.373 \\
\hline Fault current (kA) & 3.007 & 3.085 \\
\hline Voltage-to-ground during fault (p.u.) & 0.000 & 0.000 \\
\hline Fault contribution from Bus 15 (p.u.) & 5.554 & 5.739 \\
\hline Fault contribution from Bus 15 (kA) & 2.324 & 2.401 \\
\hline Voltage-to-ground at Bus 15 (p.u.) & 0.815 & 0.842 \\
\hline Fault contribution from motors (p.u.) & 1.634 & 1.634 \\
\hline Fault contribution from motors (kA) & 0.684 & 0.684 \\
\hline Fault contribution from GEN 2 (p.u.) & 1.702 & 2.500 \\
\hline Fault contribution from GEN 2 (kA) & 0.712 & 1.046 \\
\hline
\end{tabular}

Table 5.17: Comparison of fault current contributions from a 12.5 MVA synchronous generator and a 12.5 MVA series converter-connected generator with a grid-side inverter. 


\subsection{Chapter Summary}

Based on the testing results presented in this chapter, it can be concluded that the desired inverter fault current contribution, given by $\left|\mathbf{I}_{i(\mathrm{sc})}\right|=\alpha\left|\mathbf{I}_{i \text { (rated) }}\right|$, was successfully obtained by modelling the series converter-connected generator with a grid-side inverter (including the inverter transformer) by an equivalent voltage source behind an inductive reactance using a Norton-Thevenin source transformation such that conventional fault current calculation techniques were able to be employed. These results verify and validate the MATLAB model of the series converter-connected generator with a grid-side inverter, thereby ensuring the technical accuracy of the model within the framework of conventional fault analysis techniques. Furthermore, based on the testing results presented in this chapter, it can also be concluded that the fault current contribution from a series converter-connected generator with a grid-side inverter during a fault at the inverter transformer output terminals is significantly less than the fault current contribution from an equivalent local synchronous generator at the same fault location on the power system. However, at other fault locations on the power system, it was concluded that this is not necessarily the case and for a given fault location on the power system, the fault current contribution from the series converter-connected generator with a grid-side inverter was greater than the fault current contribution from the equivalent local synchronous generator. These results emphasize the need to develop mathematical and software simulation models that approximate the fault response of converter-based distributed energy resources within the framework of conventional fault analysis techniques and accurately simulate the fault current contributions to the electrical grid that a transmission or distribution utility needs to reflect in their connection impact assessments essential for the design and application of distribution and protective apparatuses used in these systems. 


\section{Chapter 6 - Conclusion}

\subsection{General}

In summary, electrical power systems are complex systems composed of a wide range of equipment devoted to generating, transmitting, and distributing electrical power to various consumption centers. Although these systems are designed to serve loads in a safe and reliable manner, the very complexity of these systems suggests that failures are unavoidable regardless of how carefully these systems have been designed. The feasibility of designing and operating a power system with zero failure rate is, if not realistic, economically unjustifiable. A fault in a circuit is any failure that interferes with the normal flow of current to the loads [1]. In most faults, a current path develops between two or more phases, or between one or more phases and the neutral (ground). Once this current path is established, it provides a short-circuit with relatively low impedance resulting in excessive current flows. If uncontrolled, these excessive current flows can cause service outage with accompanying downtown of equipment and associated inconvenience, interruption of essential facilities or vital services, extensive equipment damage, personnel injury or fatality, and possible fire damage [2].

Electric power systems have used and will continue to use synchronous machines in the foreseeable future for the generation of electricity. However, with emerging concerns over climate change and the need for reduced greenhouse gas emissions, together with the growing awareness of the importance of the natural environment and the depletion of the earth's non-renewable energy resources, the generation of electricity from distributed renewable energy resources such as solar photovoltaic (PV) and wind energy has begun to expand at a rapid pace. Proliferation of converter-based distributed energy resources in distribution systems has introduced new challenges in determining the maximum possible fault currents that a power system must be able to withstand without being compromised. As part of a connection impact assessment for a new generation facility, utilities require detailed information on fault characteristics of the generation sources. Without accurate data and details of the fault current contributions from series converterconnected solar PV and wind generators, it is not possible to assess the risk of connecting them and hence determining the strategy and settings of the overcurrent protective devices that protect the power system. Therefore, it is imperative to develop the mathematical and software simulation models that approximate the response of converter-based distributed energy resources during a fault on the transmission or distribution system in order to determine the fault current contributions to the electrical grid that a transmission or distribution utility needs to reflect in their connection impact assessments.

From the analysis in the report body, it was concluded that full-scale static converters effectively isolate the electrical generator and its specific electrical performance characteristics from the power system in the event of a fault on the host system. The performance of series converter-connected generators during a 
fault on the converter output terminals or elsewhere on the power system is dictated by the control strategy of the inverter. Before a fault occurs, the inverter output current is controlled both in magnitude and phase. The phase is typically set within minimum and maximum limits with respect to the zero crossing of the output voltage to ensure that the inverter reactive power output is kept within corresponding minimum and maximum limits. When a fault occurs on the power system, the inverter's inherent constant current control strategy can ensure that the inverter is able to provide a controlled fault current response during the entire fault duration. Therefore, utilities typically require a larger fault current contribution from such generators to assist power system protection in the detection of fault currents. As a result, modern grid-side inverter controls are typically designed to supply a larger and constant value of three-phase fault current that is related to the rated current by $\left|\mathbf{I}_{i \text { (sc) }}\right|=\alpha\left|\mathbf{I}_{i \text { (rated) }}\right|$, where $\alpha \leq 3$ for most current inverter designs [5] - [12]. In addition, most modern inverters are designed to continue to supply balanced three-phase currents irrespective of the degree of their voltage unbalance and are therefore considered to act as positivesequence constant current sources during the entire fault duration [5]. Furthermore, the inverter constant current control strategy ensures that the positive-sequence fault current supplied does not contain a dc component of current and is normally very fast so that the ac symmetrical component of current does not change with time during the fault, which means that the subtransient and steady-state inverter fault current contributions are equal [5]. 


\subsection{Chapter Wise Summary}

The preceding chapters and succeeding appendix comprising the entirety of the report can be summarized by the following:

The second chapter of the report, entitled "Symmetrical Faults", begins with an introduction to fault analysis, followed by a discussion of three-phase symmetrical faults and concludes with the formulation of a general procedure for calculating the voltages and fault currents at each bus and in each transmission line in a power system for symmetrical three-phase faults during the subtransient, transient, or steady-state periods. This general procedure is then illustrated by means of an example. This chapter provides the necessary background for subsequent chapters.

The third chapter of the report, entitled "Symmetrical Components and Unsymmetrical Faults", beings with an introduction to the analysis of unbalanced power systems, followed by a discussion of symmetrical components, sequence impedances and sequence networks, positive-, negative-, and zero-sequence equivalent circuits of generators, transmission lines, transformers, etc. and concludes with a discussion of single line-to-ground, line-to-line, and double line-to-ground faults on an unloaded generator which is then extended to unsymmetrical faults on more complex power systems. The relationships developed for each type of unsymmetrical fault are used to formulate a general procedure for calculating the voltages and fault currents in single line-to-ground, line-to-line, and double line-to-ground faults on a power system during the subtransient, transient, or steady-state periods. These relationships are then extended to the case where a fault occurs thorough an impedance. This chapter provides the necessary background for subsequent chapters.

The fourth chapter of the report, entitled "Power System Modelling and Fault Analysis of Converter-Based Distributed Energy Resources" begins with an introduction to the challenges imposed by the proliferation of converter-based distributed energy resources and identifies the importance of developing mathematical and software simulation models that approximate the response of converter-based distributed energy resources during a fault on the transmission or distribution system. This is subsequently followed by both a qualitative and quantitative analysis of the fault current contributions from converter-based distributed energy resources in the event of a fault on the host system (information that a transmission or distribution utility needs to reflect in their connection impact assessments). The chapter concludes with the modelling and fault analysis of converter-based distributed energy resources in the event of a fault on the dc side of a grid-side inverter.

The fifth chapter of the report, entitled "MATLAB Fault Current Analysis Program", begins with an introduction to a MATLAB program developed for calculating the voltages and fault currents at each bus 
and in each transmission line in a power system for symmetrical three-phase, single line-to-ground, line-toline, or double line-to-ground faults during the subtransient, transient, or steady-state periods. Test data is used to verify and validate the MATLAB program against published cases and other fault current analysis software programs to ensure its technical accuracy. After comparing the results, the MATLAB program is then used to perform fault analysis of a power system with converter-based distributed energy resources in order to simulate the fault current contributions to the electrical grid that a transmission or distribution utility needs to reflect in their connection impact assessments. The input files to the MATLAB program and the corresponding MATLAB Command Window outputs for each set of test data are provided in the appendix of this report.

Finally, the sixth chapter of the report is entitled "Conclusion", followed by an appendix which contains the entire code for the MATLAB program developed for calculating the voltages and fault currents at each bus and in each transmission line in a power system for symmetrical three-phase, single line-to-ground, line-toline, or double line-to-ground faults during the subtransient, transient, or steady-state periods. The input files to the MATLAB program and the corresponding MATLAB Command Window outputs for each set of test data (from Chapter 5) are also provided in the appendix of this report. 


\subsection{Contribution and Conclusions}

In this report, theoretical models for converter-based distributed energy resources that have been presented in various literary works and publications on the topic [5] - [12] are implemented using a program developed in MATLAB for performing fault current analysis of a power system using conventional fault analysis techniques, i.e., calculating the voltages and fault currents at each bus and in each transmission line in the power system for symmetrical three-phase, single line-to-ground, line-to-line, or double line-to-ground faults during the subtransient, transient, or steady-state periods. The MATLAB fault current analysis program was used to perform fault analysis of a power system with converter-based distributed energy resources in order to demonstrate how to implement these models to approximate the fault response of converter-based distributed energy resources within the framework of conventional fault analysis techniques and accurately simulate the fault current contributions to the electrical grid that a transmission or distribution utility needs to reflect in their connection impact assessments essential for the design and application of distribution and protective apparatuses used in these systems.

It was discussed that series converter-connected generators with grid-side inverters (e.g., solar PV and wind turbine generators) that deliver an increased constant current in response to the occurrence of a fault on the power system can be represented in industry-based fault studies by a positive-sequence equivalent circuit consisting of a constant current source, given by $\left|\mathbf{I}_{i(\text { (sc) }}\right|=\alpha\left|\mathbf{I}_{i(\text { rated })}\right|$, and negative- and zerosequence equivalent circuits consisting of infinite negative- and zero-sequence impedances, respectively, i.e., open-circuits. To overcome the difficulty of integrating a constant current source into the framework of conventional fault analysis, it was replaced by a voltage source behind an inductive reactance (as in the case of synchronous generators) using a Norton-Thevenin source transformation such that the conventional fault current calculation techniques described earlier in Chapters 2 and 3 may be employed. However, it was discussed that it is generally incorrect to assume that a series converter-connected generator with a grid-side inverter can be modeled by an internal generated voltage $\mathbf{E}_{A}$ behind a positivesequence impedance $Z_{1}$ such that $\left|\mathbf{I}_{i(\mathrm{sc})}\right|=\left|\mathbf{E}_{A}\right| /\left|Z_{1}\right|$. This representation will produce the correct inverter fault current contribution for one fault location only, namely at the inverter transformer output terminals. At other fault locations on the power system, the source transformation will produce a lower and incorrect inverter fault current contribution than that given by $\left|\mathbf{I}_{i(\text { sc })}\right|=\alpha\left|\mathbf{I}_{i(\text { rated })}\right|$. Since the equivalent positivesequence impedance varies with the inverter transformer terminal voltage which is a function of the fault current, the equivalent positive-sequence impedance must be determined by the iterative process described in Chapter 5 until it produces an inverter fault current contribution that converges to the desired value at all other fault locations on the power system.

Based on the testing results from Chapter 5 , it can be concluded that the desired inverter fault current contribution, given by $\left|\mathbf{I}_{i(\text { sc })}\right|=\alpha\left|\mathbf{I}_{i \text { (rated) }}\right|$, was successfully obtained by modelling the series converter- 
connected generator with a grid-side inverter (including the inverter transformer) by an equivalent voltage source behind an inductive reactance using a Norton-Thevenin source transformation such that conventional fault current calculation techniques were able to be employed. These results verify and validate the MATLAB model of the series converter-connected generator with a grid-side inverter, thereby ensuring the technical accuracy of the model within the framework of conventional fault analysis techniques. Furthermore, based on the testing results from Chapter 5, it can also be concluded that the fault current contribution from a series converter-connected generator with a grid-side inverter during a fault at the inverter transformer output terminals is significantly less than the fault current contribution from an equivalent local synchronous generator at the same fault location on the power system. However, at other fault locations on the power system, it was concluded that this is not necessarily the case and for a given fault location on the power system, the fault current contribution from the series converter-connected generator with a grid-side inverter was greater than the fault current contribution from the equivalent local synchronous generator. These results emphasize the need to develop mathematical and software simulation models that approximate the fault response of converter-based distributed energy resources within the framework of conventional fault analysis techniques and accurately simulate the fault current contributions to the electrical grid that a transmission or distribution utility needs to reflect in their connection impact assessments. 
Appendix 


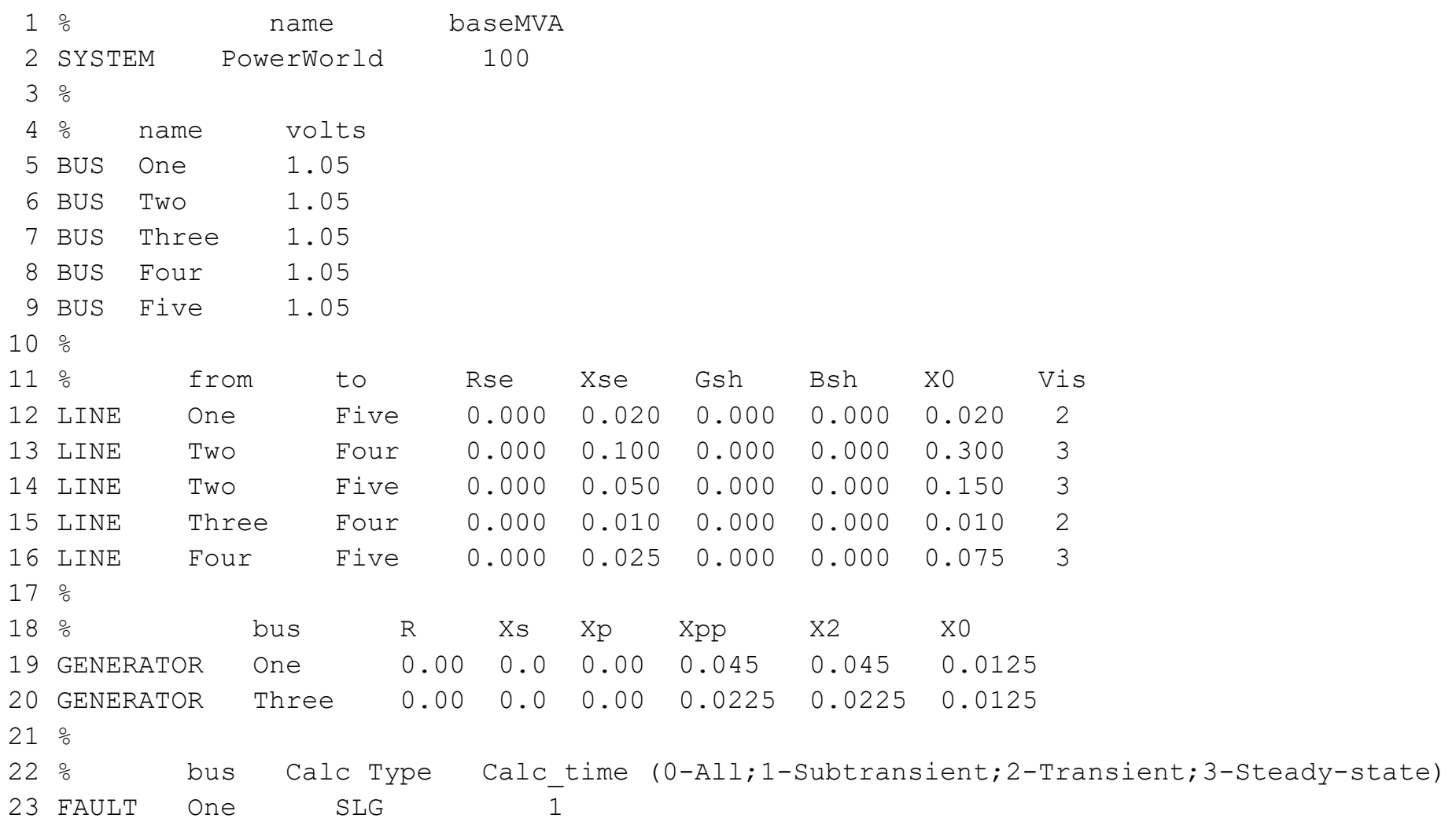




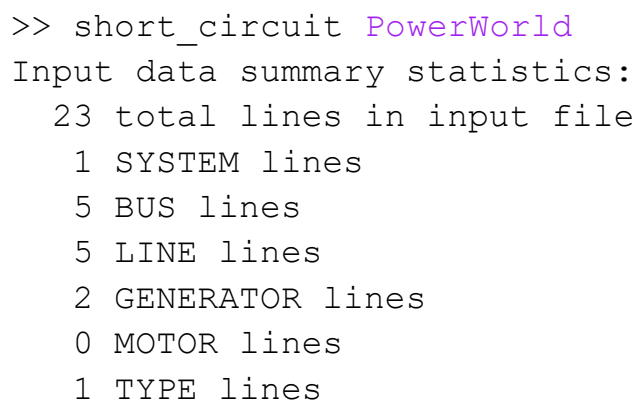

Zbus $1=$

$\begin{array}{lllll}j 0.027973 & j 0.017703 & j 0.008514 & j 0.012297 & j 0.020405 \\ j 0.017703 & j 0.056952 & j 0.013649 & j 0.019715 & j 0.025571 \\ j 0.008514 & j 0.013649 & j 0.018243 & j 0.016351 & j 0.012297 \\ j 0.012297 & j 0.019715 & j 0.016351 & j 0.023619 & j 0.017763 \\ j 0.020405 & j 0.025571 & j 0.012297 & j 0.017763 & j 0.029474\end{array}$

Zbus $2=$

$\begin{array}{lllll}j 0.027973 & j 0.017703 & j 0.008514 & j 0.012297 & j 0.020405 \\ j 0.017703 & j 0.056952 & j 0.013649 & j 0.019715 & j 0.025571 \\ j 0.008514 & j 0.013649 & j 0.018243 & j 0.016351 & j 0.012297 \\ j 0.012297 & j 0.019715 & j 0.016351 & j 0.023619 & j 0.017763 \\ j 0.020405 & j 0.025571 & j 0.012297 & j 0.017763 & j 0.029474\end{array}$

Zbus $0=$

$\begin{array}{rllll}j 0.012500 & j 0.000000 & j 0.000000 & j 0.000000 & j 0.000000 \\ j 0.000000 & j 0.108939 & j 0.000000 & j 0.004394 & j 0.011212 \\ j 0.000000 & j 0.000000 & j 0.012500 & j 0.000000 & j 0.000000 \\ j 0.000000 & j 0.004394 & j 0.000000 & j 0.008939 & j 0.002121 \\ j 0.000000 & j 0.011212 & j 0.000000 & j 0.002121 & j 0.015758 \\ & \text { Results for system Name "PowerWorld" }\end{array}$

Single Line-to-Ground Fault at Bus one

Calculating Subtransient Currents

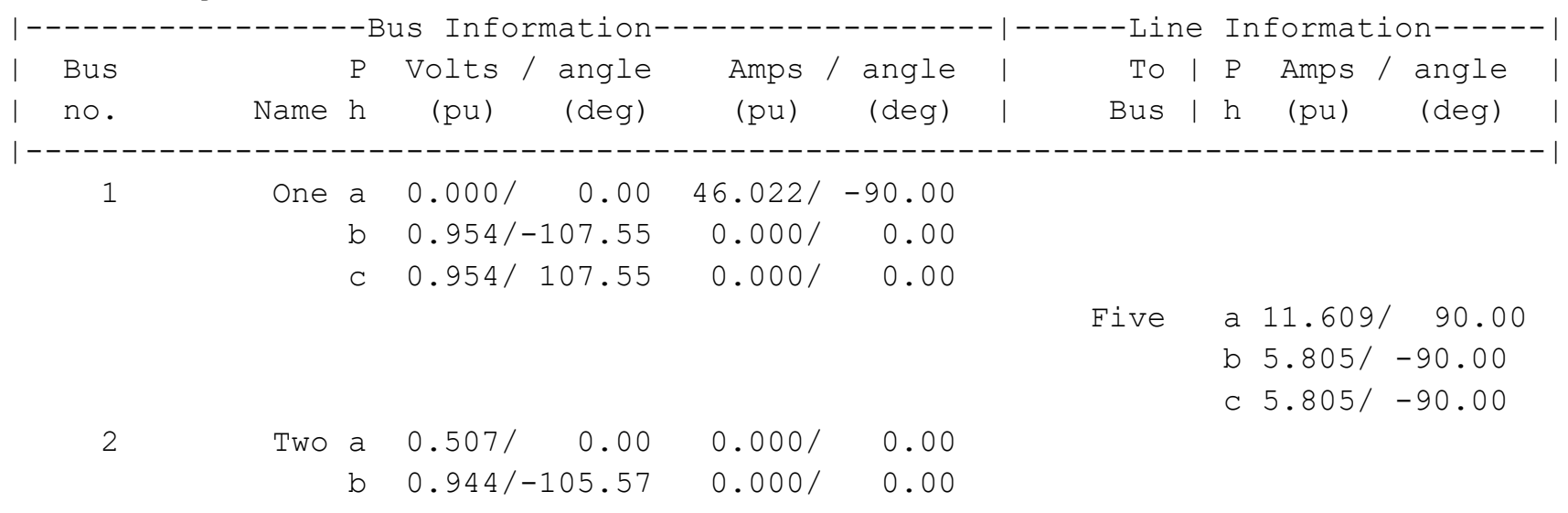


C $0.944 / 105.57 \quad 0.000 / 0.00$

Four a 1.658/90.00

b $0.829 /-90.00$

c $0.829 /-90.00$

Five a $1.658 /-90.00$

b $0.829 / 90.00$

c $0.829 / 90.00$

3

$\begin{array}{rrr}\text { Three a } & 0.789 / & 0.00 \\ \text { b } & 0.991 /-113.45 \\ \text { c } & 0.991 / 113.45\end{array}$

$0.000 / 0.00$

$0.000 / 0.00$

$0.000 / 0.00$

Four a $11.609 /-90.00$

b $5.805 / 90.00$

c $5.805 / 90.00$

4

$\begin{array}{rlrlr}\text { Four a } & 0.673 / & 0.00 & 0.000 / & 0.00 \\ \text { b } & 0.970 /-110.30 & 0.000 / & 0.00 \\ \text { c } & 0.970 / 110.30 & 0.000 / & 0.00\end{array}$

Two a $1.658 /-90.00$

b $0.829 / 90.00$

c $0.829 / 90.00$

Three a 11.609/ 90.00

b $5.805 /-90.00$

c $5.805 /-90.00$

Five a $9.951 /-90.00$

b $4.975 / 90.00$

c $4.975 / 90.00$

5
Five a $0.424 / 0.00$
$0.000 / 0.00$
b $0.934 /-103.12$
$0.000 / 0.00$
c $0.934 / 103.12$
$0.000 / 0.00$

One a $11.609 /-90.00$

b $5.805 / 90.00$

c $5.805 / 90.00$

Two a $1.658 / 90.00$

b $0.829 /-90.00$

c $0.829 /-90.00$

Four a 9.951/90.00

b $4.975 /-90.00$

c $4.975 /-90.00$ 


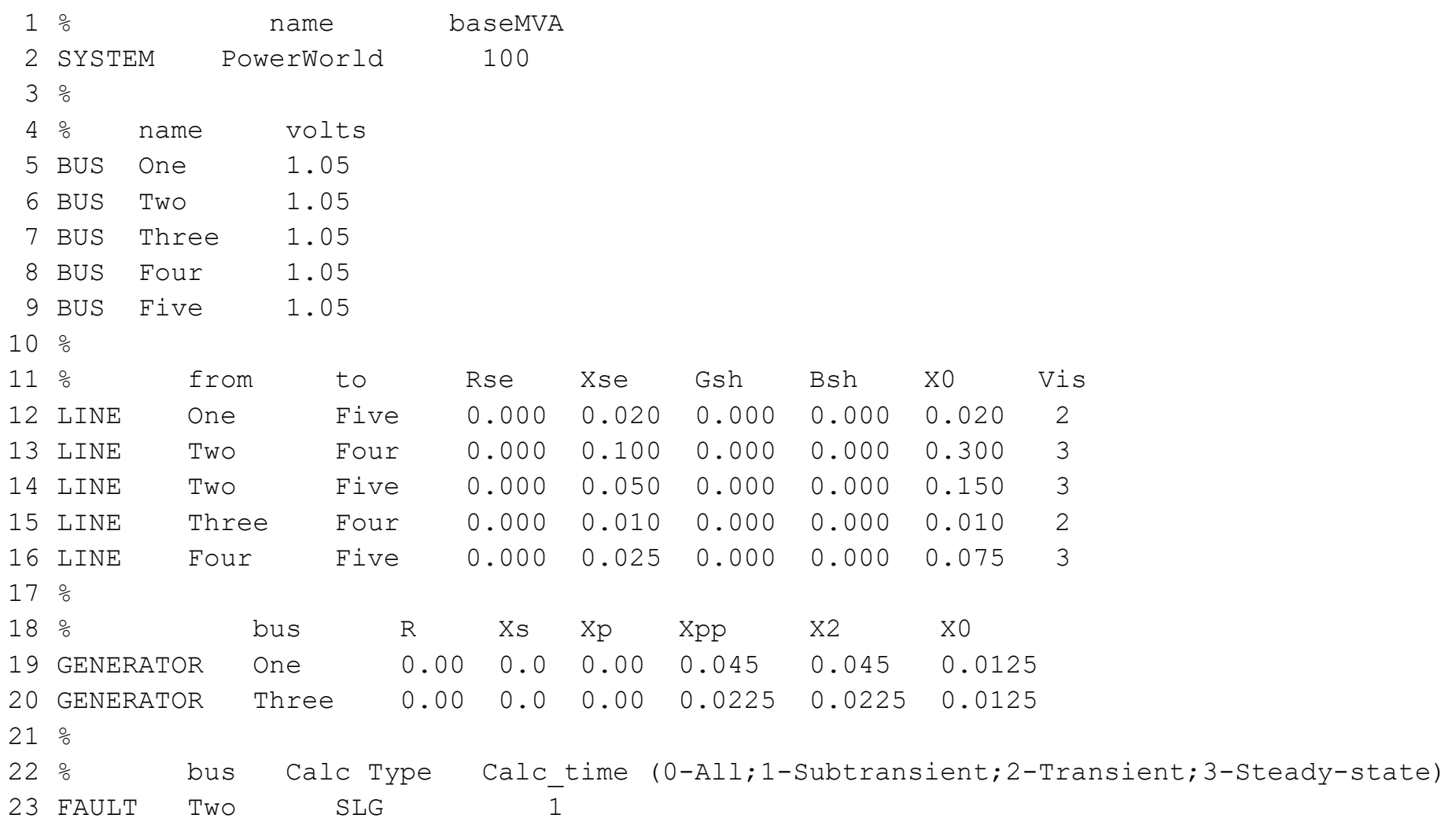




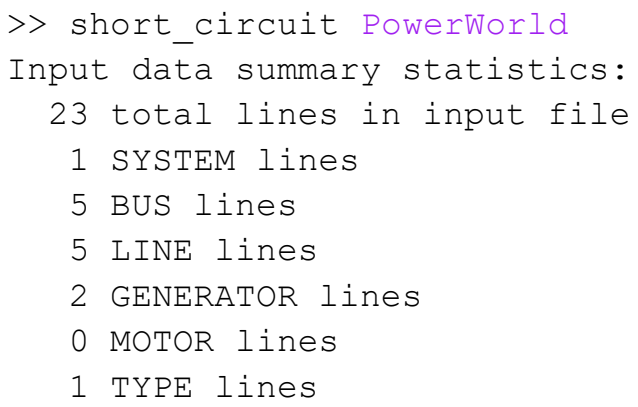




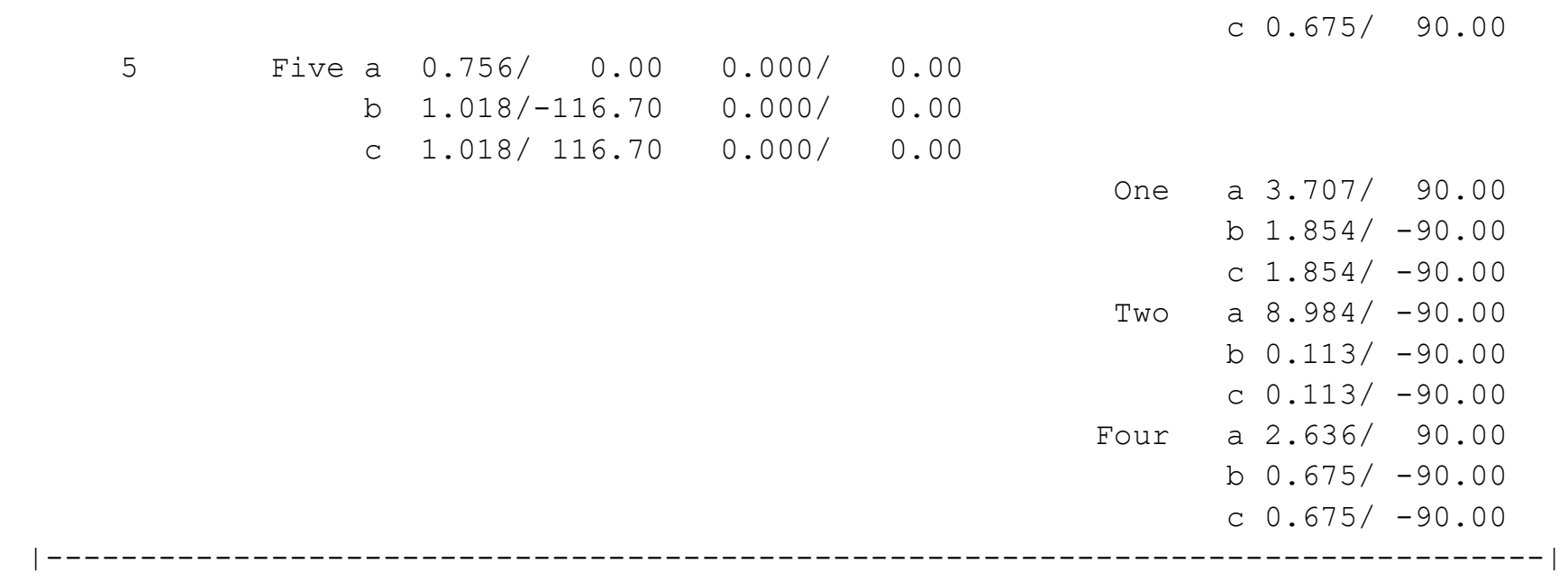




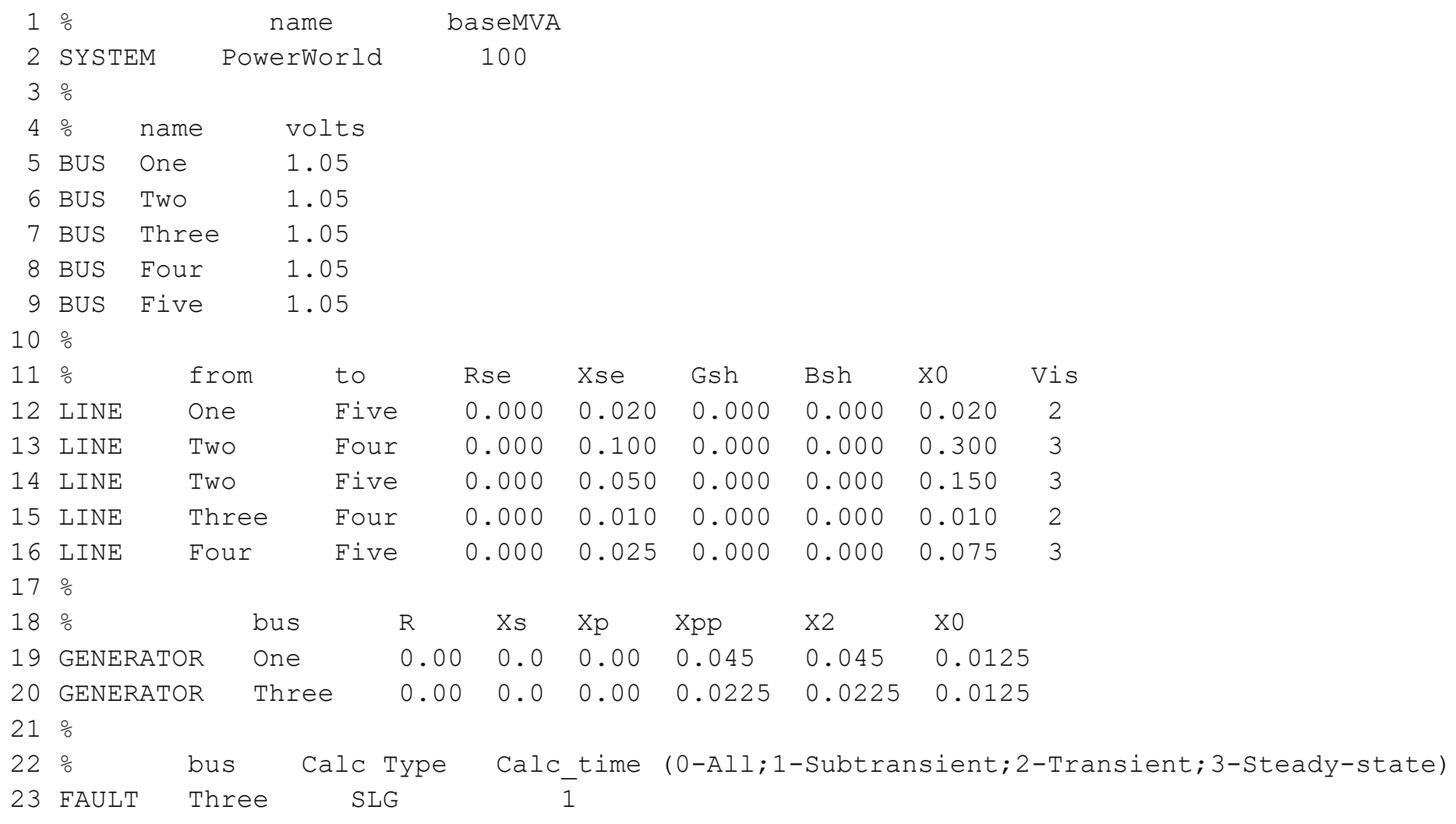




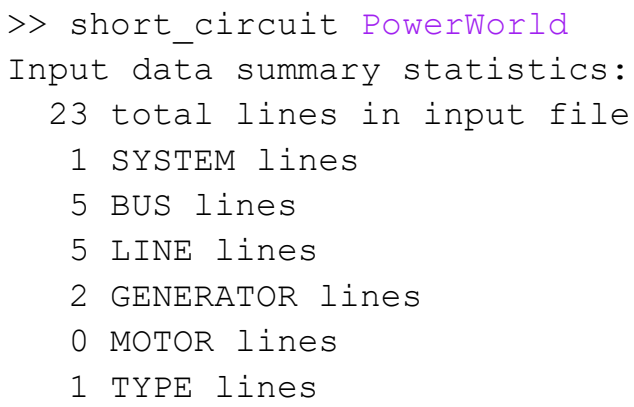

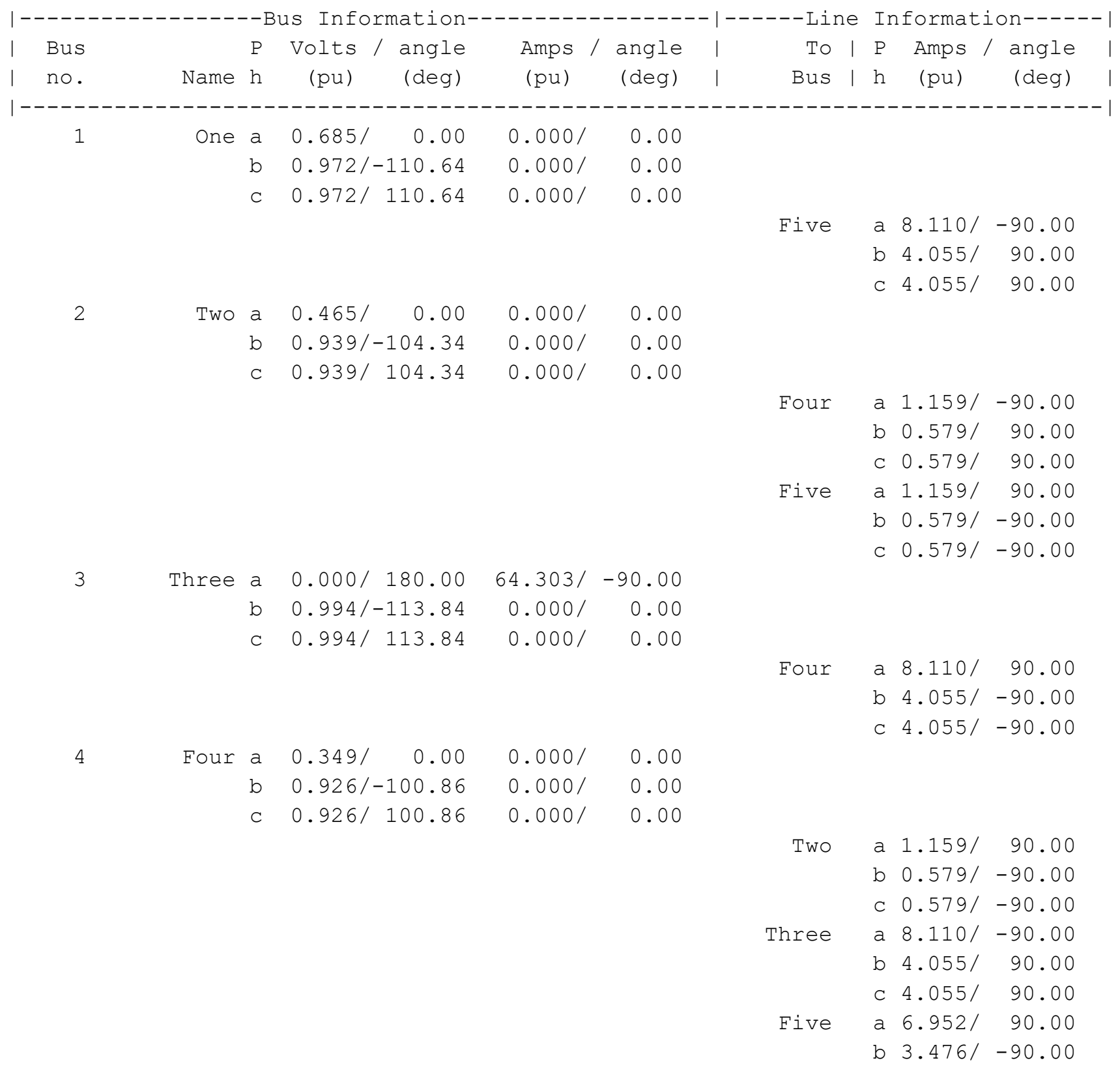




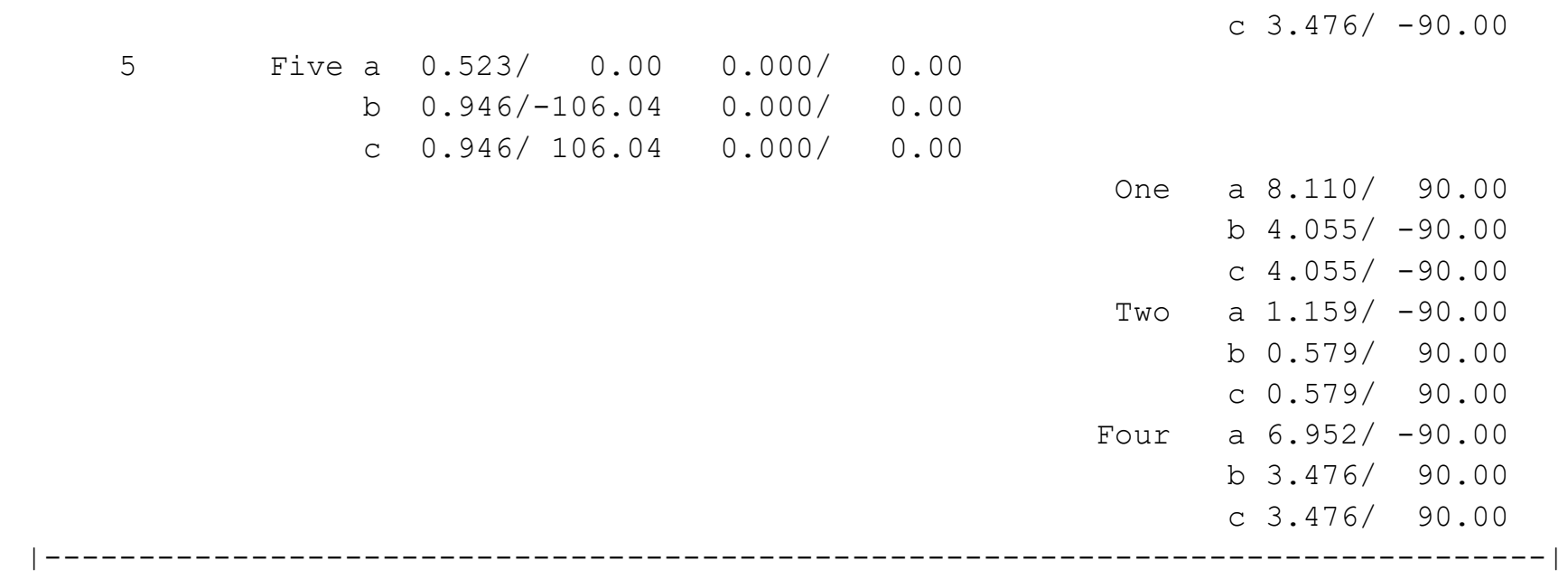




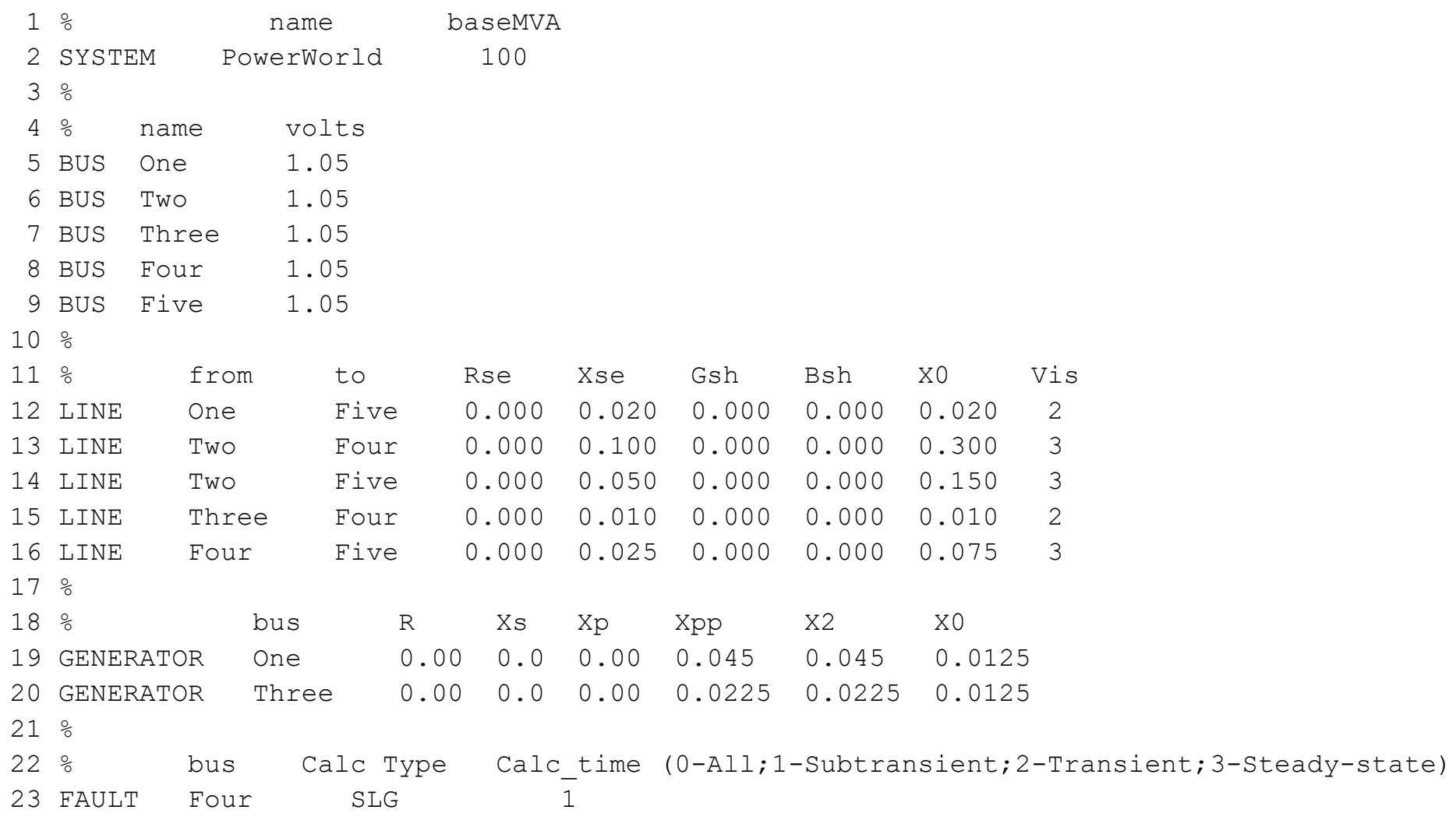




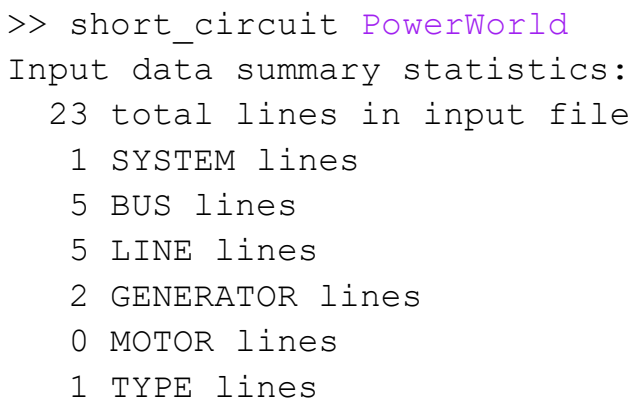

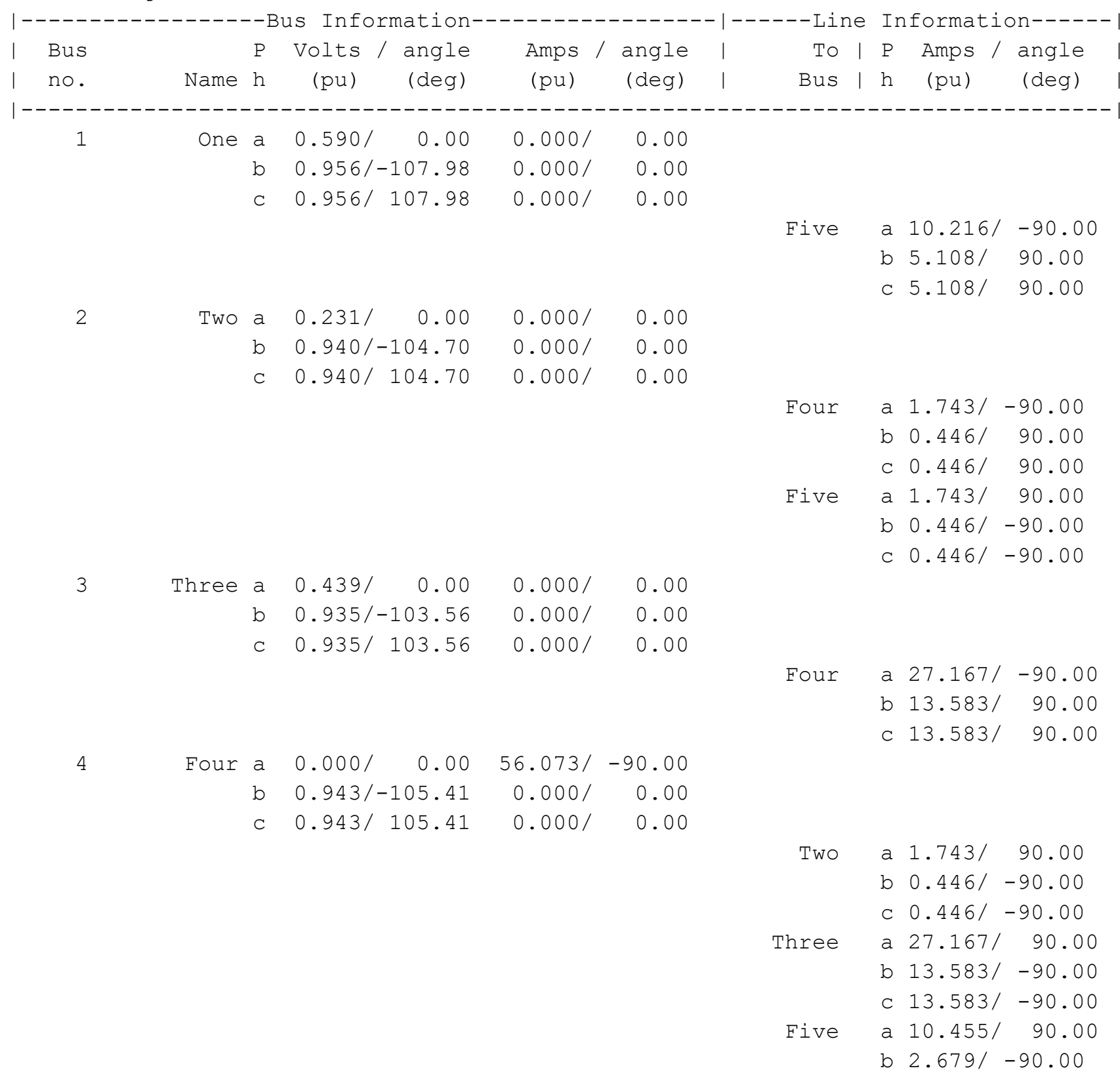




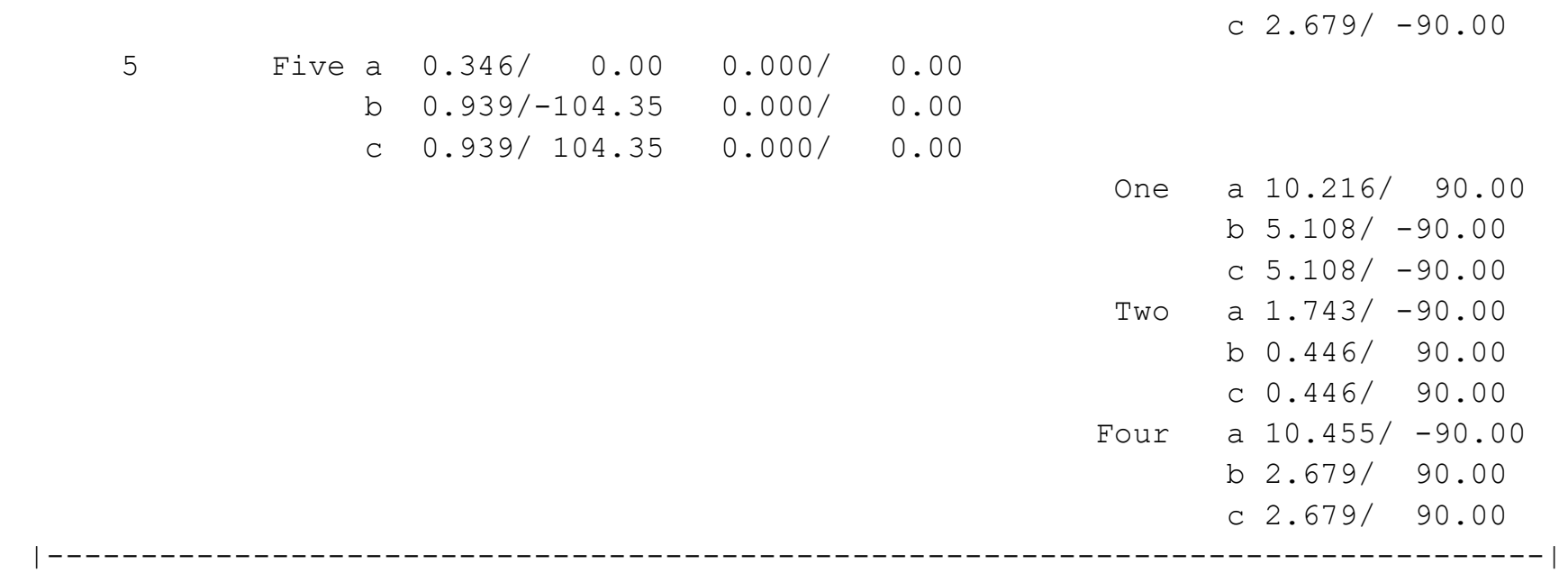




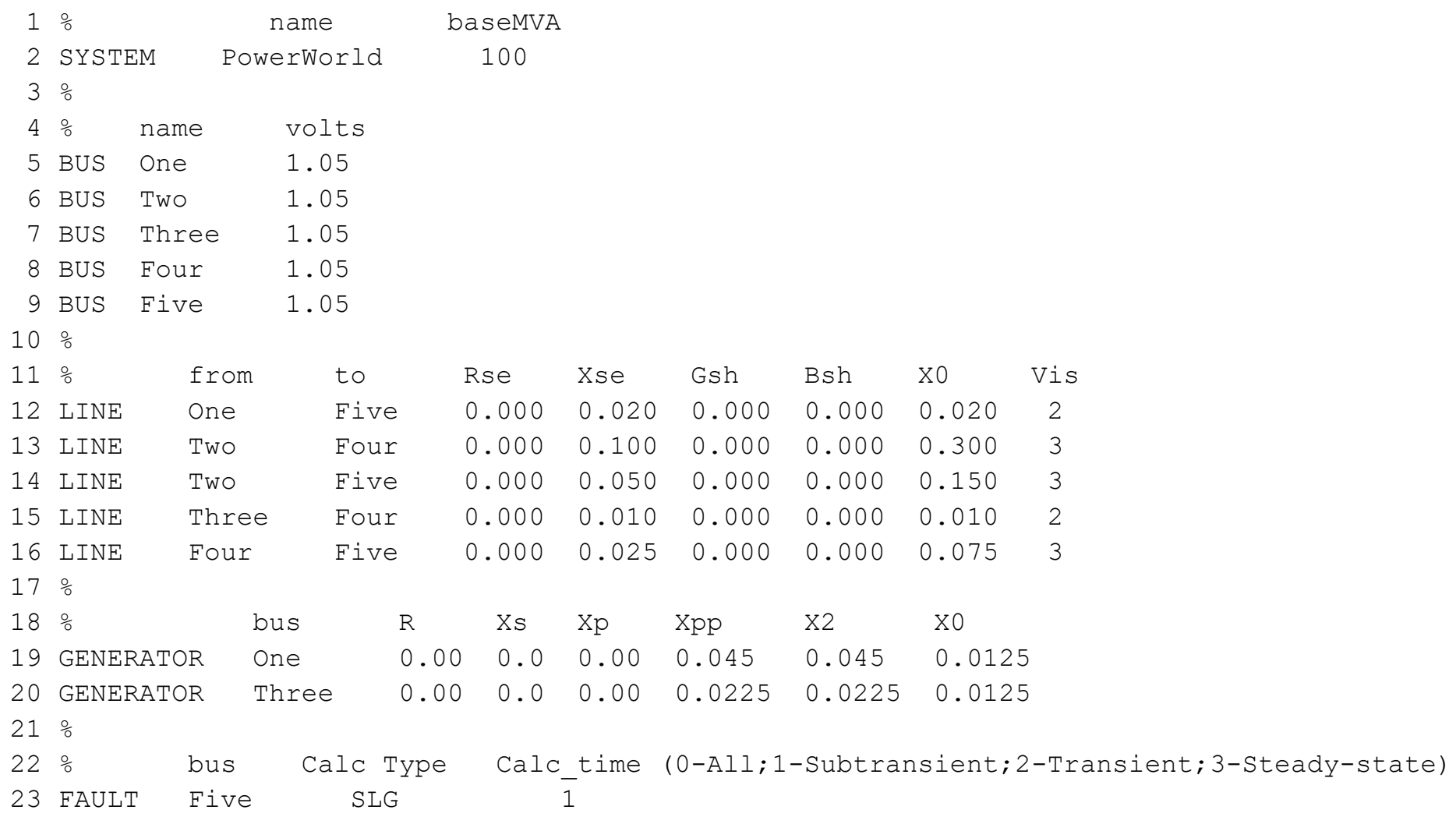




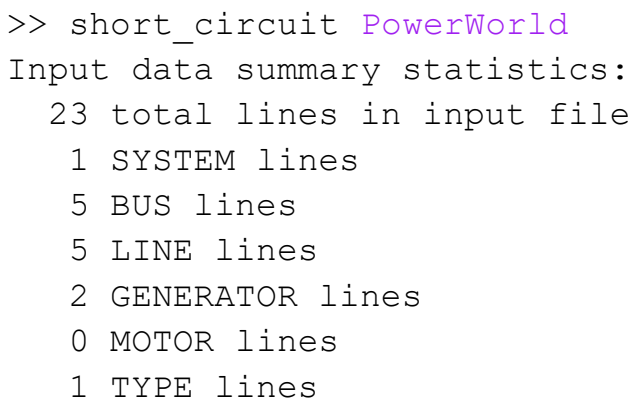

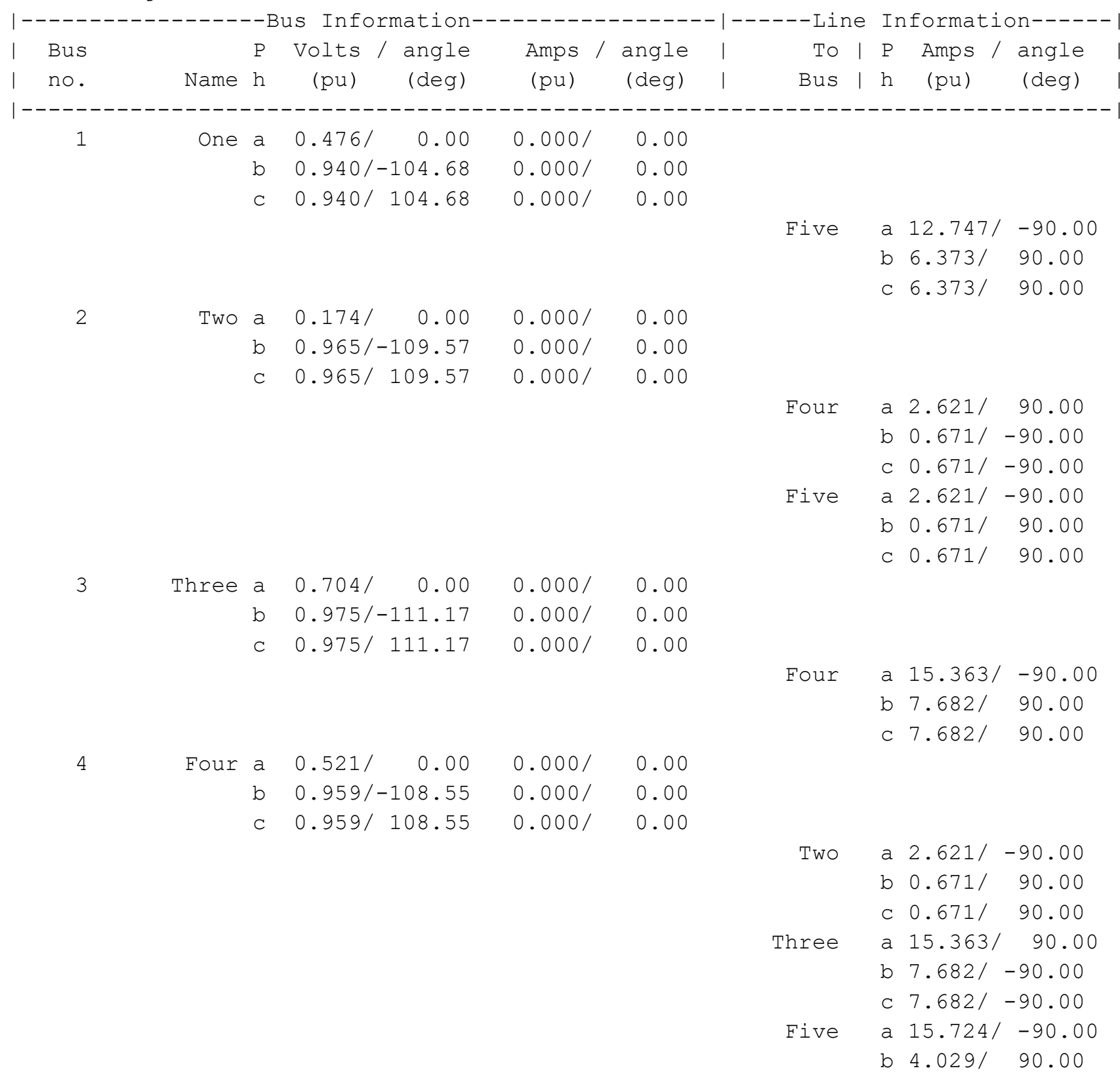




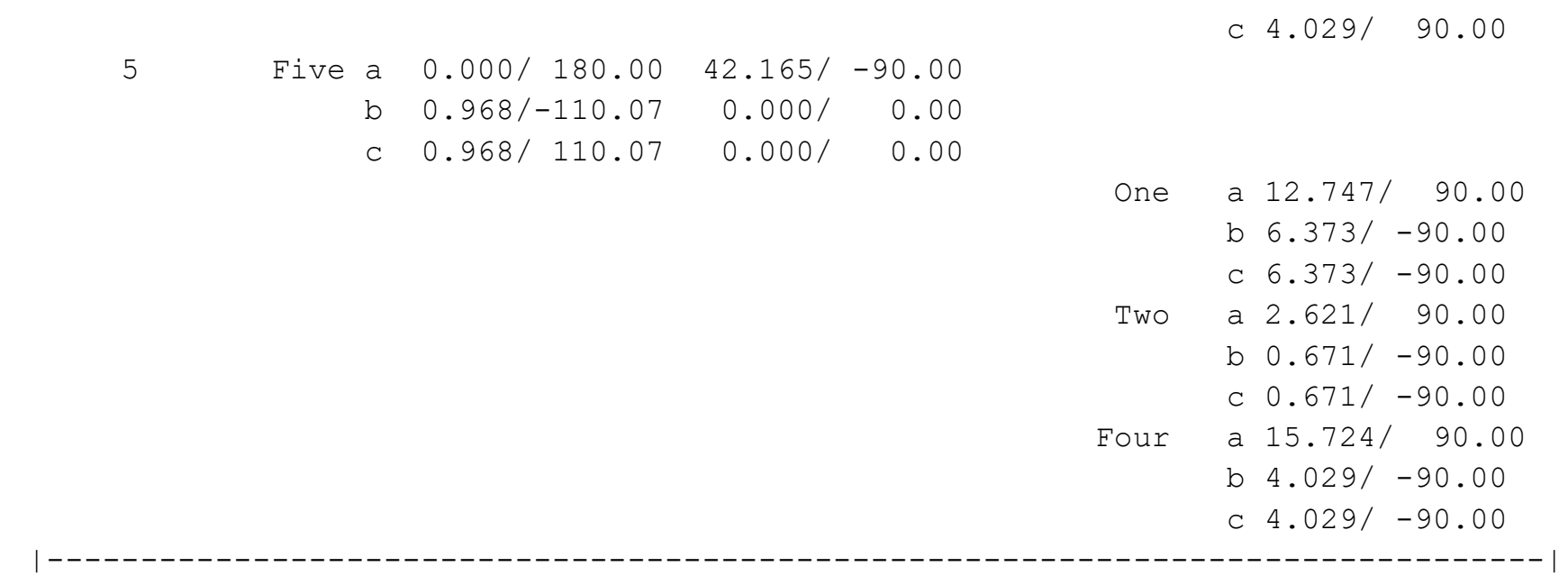




\begin{tabular}{|c|c|c|c|c|c|c|c|c|c|}
\hline \multirow{2}{*}{$\begin{array}{l}1 \\
2\end{array}$} & \multicolumn{2}{|c|}{$\%$} & name & \multicolumn{2}{|c|}{ baseMVA } & & & & \\
\hline & SYSTE & IEEE & Std_399-199 & 10 & & & & & \\
\hline 3 & $\div$ & & & & & & & & \\
\hline 4 & $\%$ & name & volts & & & & & & \\
\hline 5 & BUS & 1 & 1.00 & & & & & & \\
\hline 6 & BUS & 2 & 1.00 & & & & & & \\
\hline 7 & BUS & 3 & 1.00 & & & & & & \\
\hline 8 & BUS & 4 & 1.00 & & & & & & \\
\hline 9 & BUS & 5 & 1.00 & & & & & & \\
\hline 10 & BUS & 6 & 1.00 & & & & & & \\
\hline 11 & BUS & 8 & 1.00 & & & & & & \\
\hline 12 & BUS & 9 & 1.00 & & & & & & \\
\hline 13 & BUS & 10 & 1.00 & & & & & & \\
\hline 14 & BUS & 11 & 1.00 & & & & & & \\
\hline 15 & BUS & 12 & 1.00 & & & & & & \\
\hline 16 & BUS & 13 & 1.00 & & & & & & \\
\hline 17 & BUS & 15 & 1.00 & & & & & & \\
\hline 18 & BUS & 16 & 1.00 & & & & & & \\
\hline 19 & BUS & 17 & 1.00 & & & & & & \\
\hline 20 & BUS & 18 & 1.00 & & & & & & \\
\hline 21 & BUS & 19 & 1.00 & & & & & & \\
\hline 22 & BUS & 20 & 1.00 & & & & & & \\
\hline 23 & BUS & 21 & 1.00 & & & & & & \\
\hline 24 & BUS & 22 & 1.00 & & & & & & \\
\hline 25 & BUS & 23 & 1.00 & & & & & & \\
\hline 26 & BUS & 24 & 1.00 & & & & & & \\
\hline 27 & BUS & 25 & 1.00 & & & & & & \\
\hline 28 & BUS & 26 & 1.00 & & & & & & \\
\hline 29 & BUS & 27 & 1.00 & & & & & & \\
\hline 30 & BUS & 28 & 1.00 & & & & & & \\
\hline 31 & BUS & 29 & 1.00 & & & & & & \\
\hline 32 & BUS & 30 & 1.00 & & & & & & \\
\hline 33 & BUS & 31 & 1.00 & & & & & & \\
\hline 34 & BUS & 32 & 1.00 & & & & & & \\
\hline 35 & BUS & 33 & 1.00 & & & & & & \\
\hline 36 & BUS & 34 & 1.00 & & & & & & \\
\hline 37 & BUS & 35 & 1.00 & & & & & & \\
\hline 38 & BUS & 36 & 1.00 & & & & & & \\
\hline 39 & BUS & 37 & 1.00 & & & & & & \\
\hline 40 & BUS & 38 & 1.00 & & & & & & \\
\hline 41 & BUS & 39 & 1.00 & & & & & & \\
\hline 42 & BUS & 41 & 1.00 & & & & & & \\
\hline 43 & BUS & 49 & 1.00 & & & & & & \\
\hline 44 & BUS & 50 & 1.00 & & & & & & \\
\hline 45 & BUS & 51 & 1.00 & & & & & & \\
\hline 46 & BUS & 100 & 1.00 & & & & & & \\
\hline 47 & $\div$ & & & & & & & & \\
\hline 48 & $\frac{\circ}{0}$ & from & to & Rse & Xse & Gsh & Bsh & xo & Vis \\
\hline 49 & LINE & 1 & 3 & 0.00313 & 0.05324 & 0.00000 & 0.00000 & 0.00000 & 3 \\
\hline 50 & LINE & 2 & 4 & 0.00313 & 0.05324 & 0.00000 & 0.00000 & 0.00000 & 3 \\
\hline 51 & LINE & 5 & 39 & 0.04314 & 0.34514 & 0.00000 & 0.00000 & 0.00000 & 3 \\
\hline 52 & LINE & 5 & 49 & 0.05918 & 0.35510 & 0.00000 & 0.00000 & 0.00000 & 3 \\
\hline 53 & LINE & 6 & 11 & 0.05575 & 0.36240 & 0.00000 & 0.00000 & 0.00000 & 3 \\
\hline 54 & LINE & 6 & 19 & 0.01218 & 0.14616 & 0.00000 & 0.00000 & 0.00000 & 3 \\
\hline 55 & LINE & 12 & 17 & 0.06843 & 0.44477 & 0.00000 & 0.00000 & 0.00000 & 3 \\
\hline
\end{tabular}




\begin{tabular}{|c|c|c|c|c|c|c|c|c|}
\hline 56 & LINE & 13 & 18 & 0.05829 & 0.37888 & 0.00000 & 0.00000 & 0.00000 \\
\hline 57 & LINE & 15 & 20 & 0.01218 & 0.14616 & 0.00000 & 0.00000 & 0.00000 \\
\hline 58 & LINE & 16 & 21 & 0.15036 & 0.75178 & 0.00000 & 0.00000 & 0.00000 \\
\hline 59 & LINE & 25 & 28 & 0.05829 & 0.37888 & 0.00000 & 0.00000 & 0.00000 \\
\hline 60 & LINE & 26 & 29 & 0.05829 & 0.37888 & 0.00000 & 0.00000 & 0.00000 \\
\hline 61 & LINE & 27 & 30 & 0.05829 & 0.37888 & 0.00000 & 0.00000 & 0.00000 \\
\hline 62 & LINE & 31 & 36 & 0.02289 & 0.22886 & 0.00000 & 0.00000 & 0.00000 \\
\hline 63 & LINE & 32 & 37 & 0.10286 & 0.56573 & 0.00000 & 0.00000 & 0.00000 \\
\hline 64 & LINE & 50 & 51 & 0.06395 & 0.37796 & 0.00000 & 0.00000 & 0.00000 \\
\hline 65 & LINE & 3 & 5 & 0.00075 & 0.00063 & 0.00000 & 0.00000 & 0.00000 \\
\hline 66 & LINE & 3 & 6 & 0.00109 & 0.00091 & 0.00000 & 0.00000 & 0.00000 \\
\hline 67 & LINE & 3 & 9 & 0.00150 & 0.00125 & 0.00000 & 0.00000 & 0.00000 \\
\hline 68 & LINE & 3 & 26 & 0.00157 & 0.00131 & 0.00000 & 0.00000 & 0.00000 \\
\hline 69 & LINE & 4 & 8 & 0.00076 & 0.00092 & 0.00000 & 0.00000 & 0.00000 \\
\hline 70 & LINE & 4 & 15 & 0.00227 & 0.00189 & 0.00000 & 0.00000 & 0.00000 \\
\hline 71 & LINE & 4 & 24 & 0.00118 & 0.00098 & 0.00000 & 0.00000 & 0.00000 \\
\hline 72 & LINE & 4 & 16 & 0.00274 & 0.00229 & 0.00000 & 0.00000 & 0.00000 \\
\hline 73 & LINE & 4 & 27 & 0.00143 & 0.00119 & 0.00000 & 0.00000 & 0.00000 \\
\hline 74 & LINE & 9 & 12 & 0.00038 & 0.00032 & 0.00000 & 0.00000 & 0.00000 \\
\hline 75 & LINE & 9 & 25 & 0.00424 & 0.00353 & 0.00000 & 0.00000 & 0.00000 \\
\hline 76 & LINE & 10 & 13 & 0.00046 & 0.00039 & 0.00000 & 0.00000 & 0.00000 \\
\hline 77 & LINE & 10 & 27 & 0.00110 & 0.00091 & 0.00000 & 0.00000 & 0.00000 \\
\hline 78 & LINE & 17 & 22 & 0.03813 & 0.02451 & 0.00000 & 0.00000 & 0.00000 \\
\hline 79 & LINE & 18 & 23 & 0.03813 & 0.02451 & 0.00000 & 0.00000 & 0.00000 \\
\hline 80 & LINE & 24 & 31 & 0.00079 & 0.00065 & 0.00000 & 0.00000 & 0.00000 \\
\hline 81 & LINE & 24 & 32 & 0.00112 & 0.00093 & 0.00000 & 0.00000 & 0.00000 \\
\hline 82 & LINE & 28 & 33 & 0.03813 & 0.02451 & 0.00000 & 0.00000 & 0.00000 \\
\hline 83 & LINE & 28 & 41 & 0.03429 & 0.02105 & 0.00000 & 0.00000 & 0.00000 \\
\hline 84 & LINE & 29 & 34 & 0.03813 & 0.02451 & 0.00000 & 0.00000 & 0.00000 \\
\hline 85 & LINE & 29 & 38 & 0.08024 & 0.07732 & 0.00000 & 0.00000 & 0.00000 \\
\hline 86 & LINE & 29 & 38 & 0.08024 & 0.07732 & 0.00000 & 0.00000 & 0.00000 \\
\hline 87 & LINE & 30 & 35 & 0.03813 & 0.02451 & 0.00000 & 0.00000 & 0.00000 \\
\hline 88 & LINE & 50 & 3 & 0.00243 & 0.00485 & 0.00000 & 0.00000 & 0.00000 \\
\hline 89 & LINE & 50 & 3 & 0.00243 & 0.00485 & 0.00000 & 0.00000 & 0.00000 \\
\hline 90 & LINE & 100 & 1 & 0.00139 & 0.00296 & 0.00000 & 0.00000 & 0.00000 \\
\hline 91 & LINE & 100 & 2 & 0.00139 & 0.00296 & 0.00000 & 0.00000 & 0.00000 \\
\hline
\end{tabular}

$92 \div$

94 GENERATOR

95 GENERATOR 50

96 GENERATOR 100

$97 \%$

$98 \div$

99 MOTOR

100 MOTOR

101 MOTOR

102 MOTOR

103 MOTOR

104 MOTOR

105 MOTOR

106 MOTOR

107 MOTOR

108 MOTOR

109 MOTOR

110 MOTOR

bus
4
50
100

bus
11
17
17
18
18
19
19
20
20
21
22
23

0.003

0.002

$\mathrm{Xs}$
0.000

$\mathrm{xp}$

0.000

0.000

0.000

0.000

0.000

$\begin{array}{ll}R & X S \\ 0.352 & 0.000 \\ 0.338 & 0.000 \\ 0.802 & 0.000 \\ 0.338 & 0.000 \\ 0.802 & 0.000 \\ 0.057 & 0.000 \\ 0.047 & 0.000 \\ 0.067 & 0.000 \\ 0.060 & 0.000 \\ 0.320 & 0.000 \\ 1.398 & 0.000 \\ 1.398 & 0.000\end{array}$

Xpp
0.102

$\mathrm{x} 2$

xo

0.072

0.000

0.000

0.010

$0.000 \quad 0.000$

$0.000 \quad 0.000$

bus

17

17

18

18

19

19

20

20

21

23 $\mathrm{xp}$

0.000

Xpp

0.000

0.000

0.000

0.000

0.000

0.000

0.000

0.000

0.000

0.000

0.000

4.219

x2

0.000

$\mathrm{x} 0$

3.384

0.000

0.000

4.008

0.000

0.000

3.384

0.000

0.000

4.008

0.000

.000

1.484

0.000

0.000

0.703

0.000

0.000

1.005

0.000

.000

1.556

0.000

0.000

3.835

0.000

0.000

19.571

0.000

0.000

19.571

0.000

0.000 


\begin{tabular}{|c|c|c|c|c|c|c|c|c|}
\hline 111 MOTOR & & 28 & 0.697 & 0.000 & 0.000 & 6.972 & 0.000 & 0.000 \\
\hline 112 MOTOR & & 28 & 0.802 & 0.000 & 0.000 & 4.008 & 0.000 & 0.000 \\
\hline 13 MOTOR & & 29 & 0.321 & 0.000 & 0.000 & 3.206 & 0.000 & 0.000 \\
\hline 14 MOTOR & & 29 & 1.199 & 0.000 & 0.000 & 5.998 & 0.000 & 0.000 \\
\hline 15 MOTOR & & 30 & 0.431 & 0.000 & 0.000 & 5.166 & 0.000 & 0.000 \\
\hline $16 \mathrm{MOTOR}$ & & 30 & 1.116 & 0.000 & 0.000 & 5.578 & 0.000 & 0.000 \\
\hline 17 MOTOR & & 33 & 0.809 & 0.000 & 0.000 & 9.701 & 0.000 & 0.000 \\
\hline 18 MOTOR & & 34 & 3.621 & 0.000 & 0.000 & 25.354 & 0.000 & 0.000 \\
\hline 19 MOTOR & & 35 & 0.809 & 0.000 & 0.000 & 9.701 & 0.000 & 0.000 \\
\hline 20 MOTOR & & 36 & 0.025 & 0.000 & 0.000 & 0.818 & 0.000 & 0.000 \\
\hline 21 MOTOR & & 37 & 0.246 & 0.000 & 0.000 & 2.952 & 0.000 & 0.000 \\
\hline 22 MOTOR & & 37 & 1.859 & 0.000 & 0.000 & 9.296 & 0.000 & 0.000 \\
\hline 23 MOTOR & & 39 & 0.034 & 0.000 & 0.000 & 1.005 & 0.000 & 0.000 \\
\hline 24 MOTOR & & 49 & 0.264 & 0.000 & 0.000 & 2.640 & 0.000 & 0.000 \\
\hline 25 MOTOR & & 51 & 1.432 & 0.000 & 0.000 & 10.020 & 0.000 & 0.000 \\
\hline $126 \mathrm{MOTOR}$ & & 51 & 0.408 & 0.000 & 0.000 & 4.893 & 0.000 & 0.000 \\
\hline 27 MOTOR & & 8 & 0.006 & 0.000 & 0.000 & 0.222 & 0.000 & 0.000 \\
\hline $8 \%$ & & & & & & & & \\
\hline $9 \%$ & bus & Calc Typ & & lc_time & $(0-A)$ & 1-Subtr & noingt & 2-Transient;3-Steady-state) \\
\hline 30 FAULT & 19 & 3P & & 1 & & & & \\
\hline
\end{tabular}




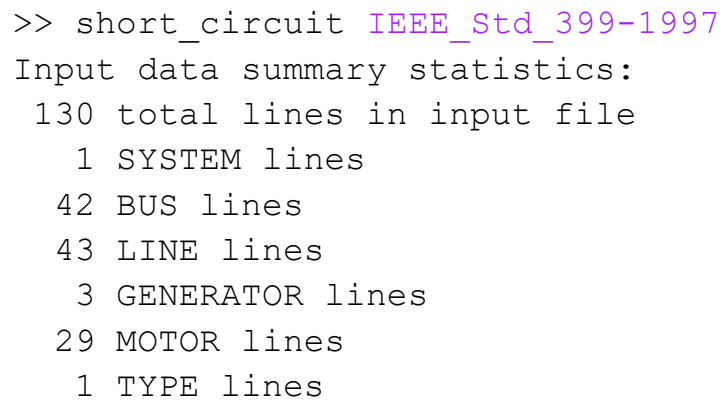

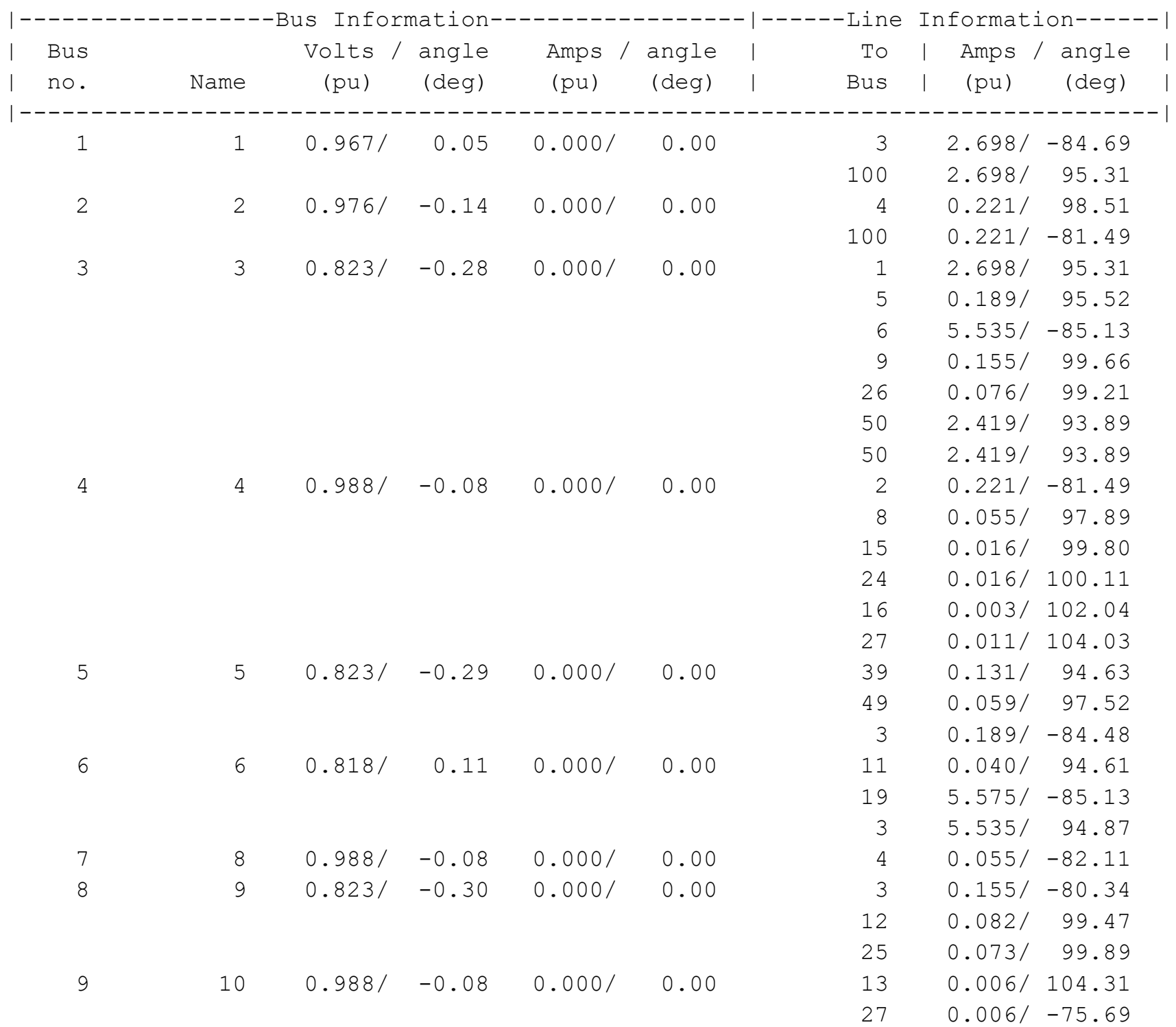




\begin{tabular}{|c|c|c|c|c|c|c|c|c|}
\hline 10 & 11 & $0.832 /$ & 0.03 & $0.000 /$ & 0.00 & 6 & $0.040 /$ & -85.39 \\
\hline \multirow[t]{2}{*}{11} & 12 & $0.823 /$ & -0.30 & $0.000 /$ & 0.00 & 17 & $0.082 /$ & 99.47 \\
\hline & & & & & & 9 & $0.082 /$ & -80.53 \\
\hline \multirow[t]{2}{*}{12} & 13 & $0.988 /$ & -0.08 & $0.000 /$ & 0.00 & 18 & $0.006 /$ & 104.31 \\
\hline & & & & & & 10 & $0.006 /$ & -75.69 \\
\hline \multirow[t]{2}{*}{13} & 15 & $0.988 /$ & -0.08 & $0.000 /$ & 0.00 & 20 & $0.016 /$ & 99.80 \\
\hline & & & & & & 4 & $0.016 /$ & -80.20 \\
\hline \multirow[t]{2}{*}{14} & 16 & $0.988 /$ & -0.08 & $0.000 /$ & 0.00 & 21 & $0.003 /$ & 102.04 \\
\hline & & & & & & 4 & $0.003 /$ & -77.96 \\
\hline \multirow[t]{2}{*}{15} & 17 & $0.860 /$ & -0.25 & $0.000 /$ & 0.00 & 12 & $0.082 /$ & -80.53 \\
\hline & & & & & & 22 & $0.007 /$ & 95.76 \\
\hline \multirow[t]{2}{*}{16} & 18 & $0.990 /$ & -0.06 & $0.000 /$ & 0.00 & 13 & $0.006 /$ & -75.69 \\
\hline & & & & & & 23 & $0.001 /$ & 100.60 \\
\hline 17 & 19 & $0.000 /$ & 0.00 & $7.667 /$ & -85.56 & 6 & $5.575 /$ & 94.87 \\
\hline 18 & 20 & $0.990 /$ & -0.07 & $0.000 /$ & 0.00 & 15 & $0.016 /$ & -80.20 \\
\hline 19 & 21 & $0.990 /$ & -0.08 & $0.000 /$ & 0.00 & 16 & $0.003 /$ & -77.96 \\
\hline 20 & 22 & $0.861 /$ & -0.27 & $0.000 /$ & 0.00 & 17 & $0.007 /$ & -84.24 \\
\hline 21 & 23 & $0.990 /$ & -0.07 & $0.000 /$ & 0.00 & 18 & $0.001 /$ & -79.40 \\
\hline \multirow[t]{3}{*}{22} & 24 & $0.988 /$ & -0.08 & $0.000 /$ & 0.00 & 4 & $0.016 /$ & -79.89 \\
\hline & & & & & & 31 & $0.012 /$ & 98.90 \\
\hline & & & & & & 32 & $0.004 /$ & 103.38 \\
\hline \multirow[t]{2}{*}{23} & 25 & $0.824 /$ & -0.31 & $0.000 /$ & 0.00 & 28 & $0.073 /$ & 99.89 \\
\hline & & & & & & 9 & $0.073 /$ & -80.11 \\
\hline \multirow[t]{2}{*}{24} & 26 & $0.823 /$ & -0.29 & $0.000 /$ & 0.00 & 29 & $0.076 /$ & 99.21 \\
\hline & & & & & & 3 & $0.076 /$ & -80.79 \\
\hline \multirow[t]{3}{*}{25} & 27 & $0.988 /$ & -0.08 & $0.000 /$ & 0.00 & 30 & $0.005 /$ & 103.70 \\
\hline & & & & & & 4 & $0.011 /$ & -75.97 \\
\hline & & & & & & 10 & $0.006 /$ & 104.31 \\
\hline \multirow[t]{3}{*}{26} & 28 & $0.852 /$ & -0.27 & $0.000 /$ & 0.00 & 25 & $0.073 /$ & -80.11 \\
\hline & & & & & & 33 & $0.015 /$ & 96.51 \\
\hline & & & & & & 41 & $0.000 /-$ & $-121 \cdot 54$ \\
\hline \multirow[t]{4}{*}{27} & 29 & $0.852 /$ & -0.26 & $0.000 /$ & 0.00 & 26 & $0.076 /$ & -80.79 \\
\hline & & & & & & 34 & $0.006 /$ & 99.73 \\
\hline & & & & & & 38 & $0.000 /-$ & -133.94 \\
\hline & & & & & & 38 & $0.000 /-$ & -133.94 \\
\hline \multirow[t]{2}{*}{28} & 30 & $0.990 /$ & -0.07 & $0.000 /$ & 0.00 & 27 & $0.005 /$ & -76.30 \\
\hline & & & & & & 35 & $0.001 /$ & 101.42 \\
\hline \multirow[t]{2}{*}{29} & 31 & $0.988 /$ & -0.08 & $0.000 /$ & 0.00 & 36 & $0.012 /$ & 98.90 \\
\hline & & & & & & 24 & $0.012 /$ & -81.10 \\
\hline \multirow[t]{2}{*}{30} & 32 & $0.988 /$ & -0.08 & $0.000 /$ & 0.00 & 37 & $0.004 /$ & 103.38 \\
\hline & & & & & & 24 & $0.004 /$ & -76.62 \\
\hline 31 & 33 & $0.852 /$ & -0.30 & $0.000 /$ & 0.00 & 28 & $0.015 /$ & -83.49 \\
\hline 32 & 34 & $0.853 /$ & -0.28 & $0.000 /$ & 0.00 & 29 & $0.006 /$ & -80.27 \\
\hline 33 & 35 & $0.990 /$ & -0.07 & $0.000 /$ & 0.00 & 30 & $0.001 /$ & -78.58 \\
\hline & 36 & $0.990 /$ & -0.07 & $0.000 /$ & 0.00 & 31 & $0.012 /$ & -81.10 \\
\hline & 37 & $0.990 /$ & -0.07 & $0.000 /$ & 0.00 & 32 & $0.004 /$ & -76.62 \\
\hline & 38 & $0.852 /$ & -0.26 & $0.000 /$ & 0.00 & 29 & $0.000 /$ & 46.06 \\
\hline
\end{tabular}




$\begin{array}{lrllll}37 & 39 & 0.869 / & -0.41 & 0.000 / & 0.00 \\ 38 & 41 & 0.852 / & -0.27 & 0.000 / & 0.00 \\ 39 & 49 & 0.844 / & -0.33 & 0.000 / & 0.00 \\ 40 & 50 & 0.829 / & -0.46 & 0.000 / & 0.00 \\ & & & & & \\ 41 & 51 & 0.847 / & -0.47 & 0.000 / & 0.00 \\ 42 & 100 & 0.975 / & -0.13 & 0.000 / & 0.00\end{array}$

$29 \quad 0.000 / 46.06$

$50.131 /-85.37$

$280.000 / 58.46$

$5 \quad 0.059 /-82.48$

$\begin{array}{lll}51 & 0.046 / 98.47\end{array}$

$32.419 /-86.11$

$32.419 /-86.11$

$50 \quad 0.046 /-81.53$

$12.698 /-84.69$

$20.221 / 98.51$

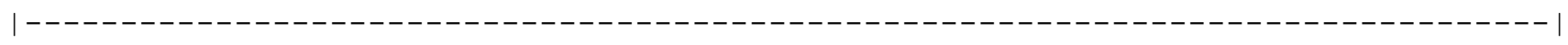

$>>$ 


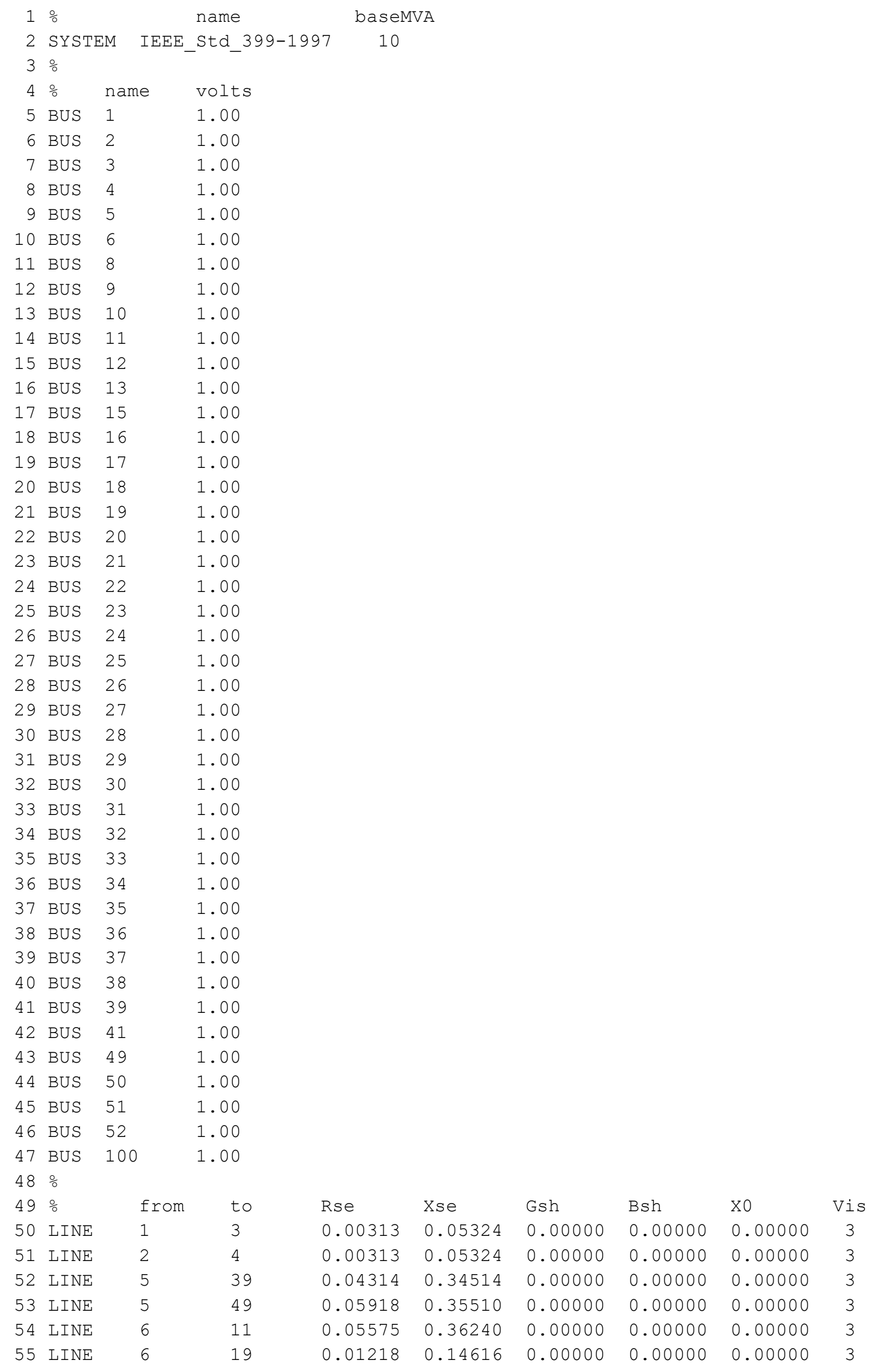




\begin{tabular}{|c|c|c|c|c|c|c|c|c|}
\hline 56 & LINE & 12 & 17 & 0.06843 & 0.44477 & 0.00000 & 0.00000 & 0.00000 \\
\hline 57 & LINE & 13 & 18 & 0.05829 & 0.37888 & 0.00000 & 0.00000 & 0.00000 \\
\hline 58 & LINE & 15 & 20 & 0.01218 & 0.14616 & 0.00000 & 0.00000 & 0.00000 \\
\hline 59 & LINE & 16 & 21 & 0.15036 & 0.75178 & 0.00000 & 0.00000 & 0.00000 \\
\hline 60 & LINE & 25 & 28 & 0.05829 & 0.37888 & 0.00000 & 0.00000 & 0.00000 \\
\hline 61 & LINE & 26 & 29 & 0.05829 & 0.37888 & 0.00000 & 0.00000 & 0.00000 \\
\hline 62 & LINE & 27 & 30 & 0.05829 & 0.37888 & 0.00000 & 0.00000 & 0.00000 \\
\hline 63 & LINE & 31 & 36 & 0.02289 & 0.22886 & 0.00000 & 0.00000 & 0.00000 \\
\hline 64 & LINE & 32 & 37 & 0.10286 & 0.56573 & 0.00000 & 0.00000 & 0.00000 \\
\hline 65 & LINE & 50 & 51 & 0.06395 & 0.37796 & 0.00000 & 0.00000 & 0.00000 \\
\hline 66 & LINE & 52 & 4 & 0.00313 & 0.05324 & 0.00000 & 0.00000 & 0.00000 \\
\hline 67 & LINE & 3 & 5 & 0.00075 & 0.00063 & 0.00000 & 0.00000 & 0.00000 \\
\hline 68 & LINE & 3 & 6 & 0.00109 & 0.00091 & 0.00000 & 0.00000 & 0.00000 \\
\hline 69 & LINE & 3 & 9 & 0.00150 & 0.00125 & 0.00000 & 0.00000 & 0.00000 \\
\hline 70 & LINE & 3 & 26 & 0.00157 & 0.00131 & 0.00000 & 0.00000 & 0.00000 \\
\hline 71 & LINE & 4 & 8 & 0.00076 & 0.00092 & 0.00000 & 0.00000 & 0.00000 \\
\hline 72 & LINE & 4 & 15 & 0.00227 & 0.00189 & 0.00000 & 0.00000 & 0.00000 \\
\hline 73 & LINE & 4 & 24 & 0.00118 & 0.00098 & 0.00000 & 0.00000 & 0.00000 \\
\hline 74 & LINE & 4 & 16 & 0.00274 & 0.00229 & 0.00000 & 0.00000 & 0.00000 \\
\hline 75 & LINE & 4 & 27 & 0.00143 & 0.00119 & 0.00000 & 0.00000 & 0.00000 \\
\hline 76 & LINE & 9 & 12 & 0.00038 & 0.00032 & 0.00000 & 0.00000 & 0.00000 \\
\hline 77 & LINE & 9 & 25 & 0.00424 & 0.00353 & 0.00000 & 0.00000 & 0.00000 \\
\hline 78 & LINE & 10 & 13 & 0.00046 & 0.00039 & 0.00000 & 0.00000 & 0.00000 \\
\hline 79 & LINE & 10 & 27 & 0.00110 & 0.00091 & 0.00000 & 0.00000 & 0.00000 \\
\hline 80 & LINE & 17 & 22 & 0.03813 & 0.02451 & 0.00000 & 0.00000 & 0.00000 \\
\hline 81 & LINE & 18 & 23 & 0.03813 & 0.02451 & 0.00000 & 0.00000 & 0.00000 \\
\hline 82 & LINE & 24 & 31 & 0.00079 & 0.00065 & 0.00000 & 0.00000 & 0.00000 \\
\hline 83 & LINE & 24 & 32 & 0.00112 & 0.00093 & 0.00000 & 0.00000 & 0.00000 \\
\hline 84 & LINE & 28 & 33 & 0.03813 & 0.02451 & 0.00000 & 0.00000 & 0.00000 \\
\hline 85 & LINE & 28 & 41 & 0.03429 & 0.02105 & 0.00000 & 0.00000 & 0.00000 \\
\hline 86 & LINE & 29 & 34 & 0.03813 & 0.02451 & 0.00000 & 0.00000 & 0.00000 \\
\hline 87 & LINE & 29 & 38 & 0.08024 & 0.07732 & 0.00000 & 0.00000 & 0.00000 \\
\hline 88 & LINE & 29 & 38 & 0.08024 & 0.07732 & 0.00000 & 0.00000 & 0.00000 \\
\hline 89 & LINE & 30 & 35 & 0.03813 & 0.02451 & 0.00000 & 0.00000 & 0.00000 \\
\hline 90 & LINE & 50 & 3 & 0.00243 & 0.00485 & 0.00000 & 0.00000 & 0.00000 \\
\hline 91 & LINE & 50 & 3 & 0.00243 & 0.00485 & 0.00000 & 0.00000 & 0.00000 \\
\hline 92 & LINE & 100 & 1 & 0.00139 & 0.00296 & 0.00000 & 0.00000 & 0.00000 \\
\hline 93 & LINE & 100 & 2 & 0.00139 & 0.00296 & 0.00000 & 0.00000 & 0.00000 \\
\hline
\end{tabular}

$94 \%$

\begin{tabular}{|c|c|}
\hline $5 \%$ & bus \\
\hline 6 GENERATOR & 50 \\
\hline GENERATOR & 52 \\
\hline 3 GENERATOR & 100 \\
\hline
\end{tabular}

$100 \%$

101 MOTOR

bus

102 MOTOR

103 MOTOR

104 MOTOR

105 MOTOR

106 MOTOR

107 MOTOR

108 MOTOR

109 MOTOR

110 MOTOR

0.002

0.000

0.000

$\mathrm{Xs}$

0.000

0.000

0.000

$\begin{array}{ll}R & X s \\ 0.352 & 0.000 \\ 0.338 & 0.000 \\ 0.802 & 0.000 \\ 0.338 & 0.000 \\ 0.802 & 0.000 \\ 0.057 & 0.000 \\ 0.047 & 0.000 \\ 0.067 & 0.000 \\ 0.060 & 0.000 \\ 0.320 & 0.000\end{array}$

$\mathrm{Xp}$

0.000

0.000

0.000

0.000

0.000

0.000

0.000

0.000

0.000

0.000

Xpp

$\mathrm{x} 2 \mathrm{x}$ 


\begin{tabular}{|c|c|c|c|c|c|c|c|c|c|}
\hline 111 & MOTOR & & 22 & 1.398 & 0.000 & 0.000 & 19.571 & 0.000 & 0.000 \\
\hline 112 & MOTOR & & 23 & 1.398 & 0.000 & 0.000 & 19.571 & 0.000 & 0.000 \\
\hline 113 & MOTOR & & 28 & 0.697 & 0.000 & 0.000 & 6.972 & 0.000 & 0.000 \\
\hline 114 & MOTOR & & 28 & 0.802 & 0.000 & 0.000 & 4.008 & 0.000 & 0.000 \\
\hline 115 & MOTOR & & 29 & 0.321 & 0.000 & 0.000 & 3.206 & 0.000 & 0.000 \\
\hline 116 & MOTOR & & 29 & 1.199 & 0.000 & 0.000 & 5.998 & 0.000 & 0.000 \\
\hline 117 & MOTOR & & 30 & 0.431 & 0.000 & 0.000 & 5.166 & 0.000 & 0.000 \\
\hline 118 & MOTOR & & 30 & 1.116 & 0.000 & 0.000 & 5.578 & 0.000 & 0.000 \\
\hline 119 & MOTOR & & 33 & 0.809 & 0.000 & 0.000 & 9.701 & 0.000 & 0.000 \\
\hline 120 & MOTOR & & 34 & 3.621 & 0.000 & 0.000 & 25.354 & 0.000 & 0.000 \\
\hline 121 & MOTOR & & 35 & 0.809 & 0.000 & 0.000 & 9.701 & 0.000 & 0.000 \\
\hline 122 & MOTOR & & 36 & 0.025 & 0.000 & 0.000 & 0.818 & 0.000 & 0.000 \\
\hline 123 & MOTOR & & 37 & 0.246 & 0.000 & 0.000 & 2.952 & 0.000 & 0.000 \\
\hline 124 & MOTOR & & 37 & 1.859 & 0.000 & 0.000 & 9.296 & 0.000 & 0.000 \\
\hline 125 & MOTOR & & 39 & 0.034 & 0.000 & 0.000 & 1.005 & 0.000 & 0.000 \\
\hline 126 & MOTOR & & 49 & 0.264 & 0.000 & 0.000 & 2.640 & 0.000 & 0.000 \\
\hline 127 & MOTOR & & 51 & 1.432 & 0.000 & 0.000 & 10.020 & 0.000 & 0.000 \\
\hline 128 & MOTOR & & 51 & 0.408 & 0.000 & 0.000 & 4.893 & 0.000 & 0.000 \\
\hline 129 & MOTOR & & 8 & 0.006 & 0.000 & 0.000 & 0.222 & 0.000 & 0.000 \\
\hline 30 & $\%$ & & & & & & & & \\
\hline & $\%$ & bus & Calc & Type & c_time & & & & 2-Transient;3-Steady-state) \\
\hline 132 & FAULT & 4 & $3 \mathrm{E}$ & & 1 & & & & \\
\hline
\end{tabular}




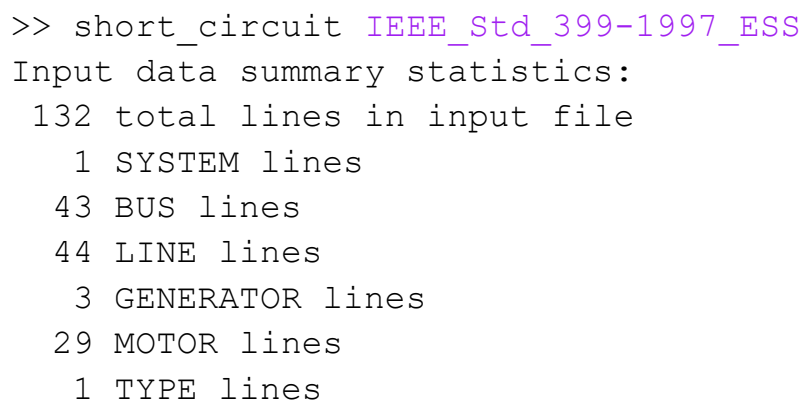

Results for System Name "IEEE_Std_399-1997"

Symmetrical Three-Phase Fault at Bus 4

Calculating Subtransient Currents

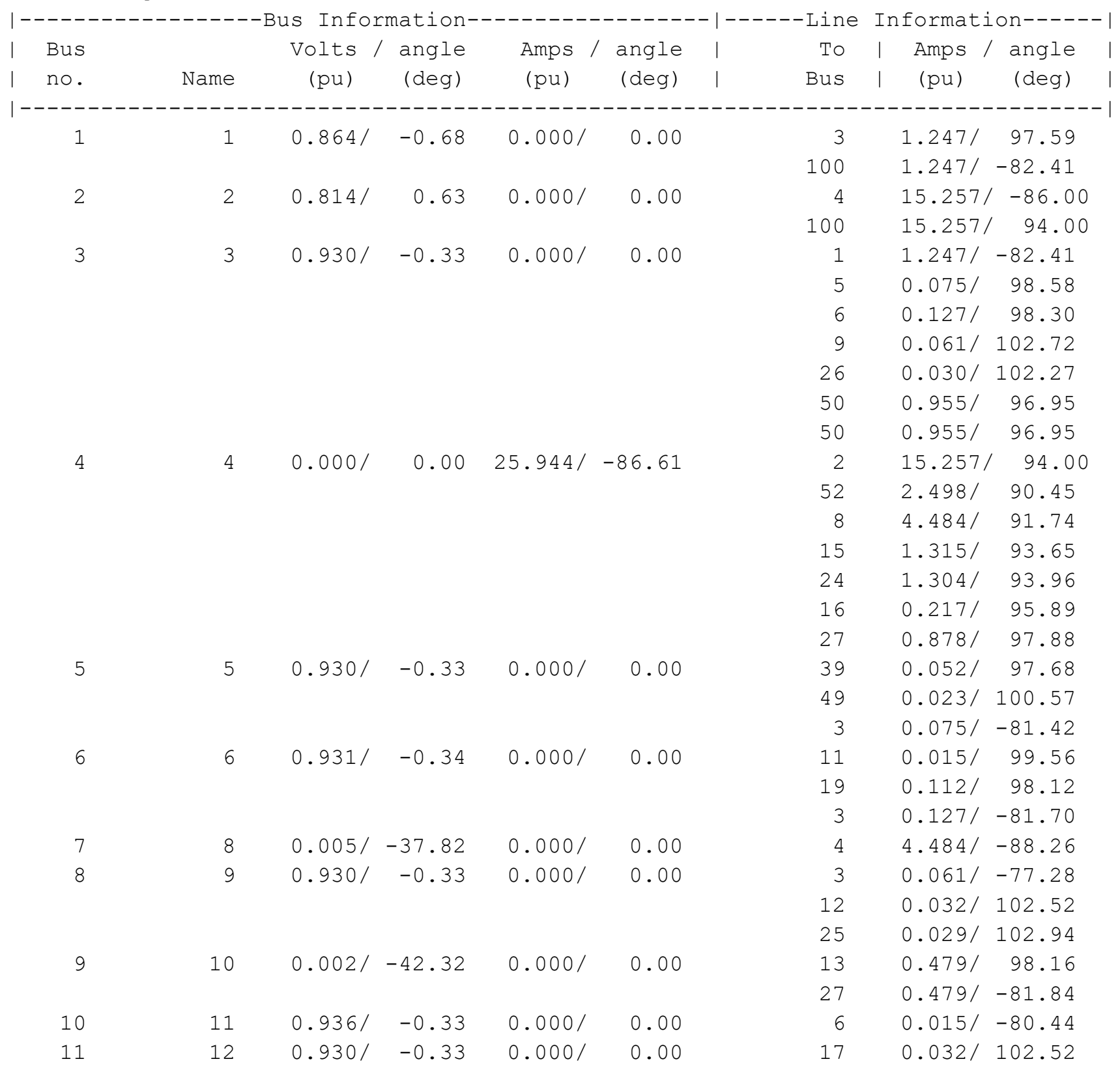




\begin{tabular}{|c|c|c|c|c|c|c|c|c|}
\hline 12 & 13 & $0.003 /$ & -42.24 & $0.000 /$ & 0.00 & $\begin{array}{l}18 \\
10\end{array}$ & $\begin{array}{l}0.479 / \\
0.479 /\end{array}$ & $\begin{array}{r}98.16 \\
-81.84\end{array}$ \\
\hline 13 & 15 & $0.004 /$ & -46.57 & $0.000 /$ & 0.00 & $\begin{array}{r}20 \\
4\end{array}$ & $1.315 /$ & $\begin{array}{r}93.65 \\
-86.35\end{array}$ \\
\hline 14 & 16 & $0.001 /$ & -44.23 & $0.000 /$ & 0.00 & $\begin{array}{r}21 \\
4\end{array}$ & $\begin{array}{l}0.217 / \\
0.217 /\end{array}$ & $\begin{array}{r}95.89 \\
-84.11\end{array}$ \\
\hline 15 & 17 & $0.945 /$ & -0.27 & $0.000 /$ & 0.00 & $\begin{array}{l}12 \\
22\end{array}$ & $\begin{array}{l}0.032 / \\
0.003 /\end{array}$ & $\begin{array}{r}-77.48 \\
98.81\end{array}$ \\
\hline 16 & 18 & $0.186 /$ & -1.12 & $0.000 /$ & 0.00 & $\begin{array}{l}13 \\
23\end{array}$ & $\begin{array}{l}0.479 / \\
0.041 /\end{array}$ & $\begin{array}{r}-81.84 \\
94.45\end{array}$ \\
\hline 17 & 19 & $0.947 /$ & -0.27 & $0.000 /$ & 0.00 & 6 & $0.112 /$ & -81.88 \\
\hline 18 & 20 & $0.196 /$ & -1.92 & $0.000 /$ & 0.00 & 15 & $1.315 /$ & -86.35 \\
\hline 19 & 21 & $0.167 /$ & -5.59 & $0.000 /$ & 0.00 & 16 & $0.217 /$ & -84.11 \\
\hline 20 & 22 & $0.945 /$ & -0.28 & $0.000 /$ & 0.00 & 17 & $0.003 /$ & -81.19 \\
\hline 21 & 23 & $0.187 /$ & -1.57 & $0.000 /$ & 0.00 & 18 & $0.041 /$ & -85.55 \\
\hline 22 & 24 & $0.002 /$ & -46.33 & $0.000 /$ & 0.00 & $\begin{array}{r}4 \\
31 \\
32\end{array}$ & $\begin{array}{l}1.304 / \\
0.952 / \\
0.352 /\end{array}$ & $\begin{array}{r}-86.04 \\
92.74 \\
97.23\end{array}$ \\
\hline 23 & 25 & $0.931 /$ & -0.34 & $0.000 /$ & 0.00 & $\begin{array}{r}28 \\
9\end{array}$ & $\begin{array}{l}0.029 / \\
0.029 /\end{array}$ & $\begin{array}{r}102.94 \\
-77.06\end{array}$ \\
\hline 24 & 26 & $0.930 /$ & -0.33 & $0.000 /$ & 0.00 & $\begin{array}{r}29 \\
3\end{array}$ & $\begin{array}{l}0.030 / \\
0.030 /\end{array}$ & $\begin{array}{r}102.27 \\
-77.73\end{array}$ \\
\hline 25 & 27 & $0.002 /$ & -42.35 & $0.000 /$ & 0.00 & $\begin{array}{r}30 \\
4 \\
10\end{array}$ & $\begin{array}{l}0.398 / \\
0.878 / \\
0.479 /\end{array}$ & $\begin{array}{r}97.55 \\
-82.12 \\
98.16\end{array}$ \\
\hline 26 & 28 & $0.942 /$ & -0.29 & $0.000 /$ & 0.00 & $\begin{array}{l}25 \\
33 \\
41\end{array}$ & $\begin{array}{l}0.029 / \\
0.006 / \\
0.000 /-\end{array}$ & $\begin{array}{r}-77.06 \\
99.56 \\
-121.54\end{array}$ \\
\hline 27 & 29 & $0.942 /$ & -0.28 & $0.000 /$ & 0.00 & $\begin{array}{l}26 \\
34 \\
38 \\
38\end{array}$ & $\begin{array}{l}0.030 / \\
0.002 / \\
0.000 /- \\
0.000 /-\end{array}$ & $\begin{array}{r}-77.73 \\
102.78 \\
-133.94 \\
-133.94\end{array}$ \\
\hline 28 & 30 & $0.154 /$ & -1.60 & $0.000 /$ & 0.00 & $\begin{array}{l}27 \\
35\end{array}$ & $\begin{array}{l}0.398 / / \\
0.087 /\end{array}$ & $\begin{array}{r}-82.45 \\
95.27\end{array}$ \\
\hline 29 & 31 & $0.003 /$ & -46.82 & $0.000 /$ & 0.00 & $\begin{array}{l}36 \\
24\end{array}$ & $\begin{array}{l}0.952 / \\
0.952 /\end{array}$ & $\begin{array}{r}92.74 \\
-87.26\end{array}$ \\
\hline 30 & 32 & $0.003 /$ & -45.67 & $0.000 /$ & 0.00 & $\begin{array}{l}37 \\
24\end{array}$ & $\begin{array}{l}0.352 / \\
0.352 /\end{array}$ & $\begin{array}{r}97.23 \\
-82.77\end{array}$ \\
\hline 31 & 33 & $0.942 /$ & -0.30 & $0.000 /$ & 0.00 & 28 & $0.006 /$ & -80.44 \\
\hline 32 & 34 & $0.942 /$ & -0.29 & $0.000 /$ & 0.00 & 29 & $0.002 /$ & -77.22 \\
\hline 33 & 35 & $0.156 /$ & -2.71 & $0.000 /$ & 0.00 & 30 & $0.087 /$ & -84.73 \\
\hline 34 & 36 & $0.221 /$ & -3.50 & $0.000 /$ & 0.00 & 31 & $0.952 /$ & -87.26 \\
\hline 35 & 37 & $0.204 /$ & -3.55 & $0.000 /$ & 0.00 & 32 & $0.352 /$ & -82.77 \\
\hline 36 & 38 & $0.942 /$ & -0.28 & $0.000 /$ & 0.00 & $\begin{array}{l}29 \\
29\end{array}$ & $\begin{array}{l}0.000 / \\
0.000 / /\end{array}$ & $\begin{array}{l}46.06 \\
46.06\end{array}$ \\
\hline 37 & 39 & $0.948 /$ & -0.31 & $0.000 /$ & 0.00 & 5 & $0.052 /$ & -82.32 \\
\hline 38 & 41 & $0.942 /$ & -0.29 & $0.000 /$ & 0.00 & 28 & $0.000 /$ & 58.46 \\
\hline 39 & 49 & $0.939 /$ & -0.32 & $0.000 /$ & 0.00 & 5 & $0.023 /$ & -79.43 \\
\hline 60 & 50 & $0.933 /$ & -0.38 & $0.000 /$ & 0.00 & 51 & $0.018 /$ & 101.52 \\
\hline
\end{tabular}




\begin{tabular}{|c|c|}
\hline 52 & $0.133 /$ \\
\hline 100 & $0.860 /$ \\
\hline
\end{tabular}

$30.955 /-83.05$

$30.955 /-83.05$

41

43

$0.000 /$

50

$0.018 /-78.48$

$4 \quad 2.498 /-89.55$

$1 \quad 1.247 / 97.59$

$2 \quad 15.257 /-86.00$

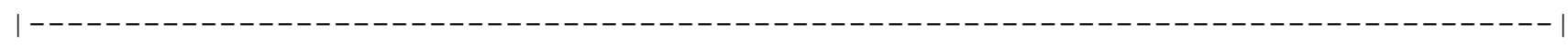




\begin{tabular}{|c|c|c|c|c|c|c|c|c|c|}
\hline \multirow{2}{*}{$\begin{array}{l}1 \\
2\end{array}$} & \multicolumn{2}{|c|}{$\%$} & name & \multicolumn{2}{|c|}{ baseMVA } & & & & \\
\hline & SYSTE & IEEE & Std_399-199 & 10 & & & & & \\
\hline 3 & $\div$ & & & & & & & & \\
\hline 4 & $\%$ & name & volts & & & & & & \\
\hline 5 & BUS & 1 & 1.00 & & & & & & \\
\hline 6 & BUS & 2 & 1.00 & & & & & & \\
\hline 7 & BUS & 3 & 1.00 & & & & & & \\
\hline 8 & BUS & 4 & 1.00 & & & & & & \\
\hline 9 & BUS & 5 & 1.00 & & & & & & \\
\hline 10 & BUS & 6 & 1.00 & & & & & & \\
\hline 11 & BUS & 8 & 1.00 & & & & & & \\
\hline 12 & BUS & 9 & 1.00 & & & & & & \\
\hline 13 & BUS & 10 & 1.00 & & & & & & \\
\hline 14 & BUS & 11 & 1.00 & & & & & & \\
\hline 15 & BUS & 12 & 1.00 & & & & & & \\
\hline 16 & BUS & 13 & 1.00 & & & & & & \\
\hline 17 & BUS & 15 & 1.00 & & & & & & \\
\hline 18 & BUS & 16 & 1.00 & & & & & & \\
\hline 19 & BUS & 17 & 1.00 & & & & & & \\
\hline 20 & BUS & 18 & 1.00 & & & & & & \\
\hline 21 & BUS & 19 & 1.00 & & & & & & \\
\hline 22 & BUS & 20 & 1.00 & & & & & & \\
\hline 23 & BUS & 21 & 1.00 & & & & & & \\
\hline 24 & BUS & 22 & 1.00 & & & & & & \\
\hline 25 & BUS & 23 & 1.00 & & & & & & \\
\hline 26 & BUS & 24 & 1.00 & & & & & & \\
\hline 27 & BUS & 25 & 1.00 & & & & & & \\
\hline 28 & BUS & 26 & 1.00 & & & & & & \\
\hline 29 & BUS & 27 & 1.00 & & & & & & \\
\hline 30 & BUS & 28 & 1.00 & & & & & & \\
\hline 31 & BUS & 29 & 1.00 & & & & & & \\
\hline 32 & BUS & 30 & 1.00 & & & & & & \\
\hline 33 & BUS & 31 & 1.00 & & & & & & \\
\hline 34 & BUS & 32 & 1.00 & & & & & & \\
\hline 35 & BUS & 33 & 1.00 & & & & & & \\
\hline 36 & BUS & 34 & 1.00 & & & & & & \\
\hline 37 & BUS & 35 & 1.00 & & & & & & \\
\hline 38 & BUS & 36 & 1.00 & & & & & & \\
\hline 39 & BUS & 37 & 1.00 & & & & & & \\
\hline 40 & BUS & 38 & 1.00 & & & & & & \\
\hline 41 & BUS & 39 & 1.00 & & & & & & \\
\hline 42 & BUS & 41 & 1.00 & & & & & & \\
\hline 43 & BUS & 49 & 1.00 & & & & & & \\
\hline 44 & BUS & 50 & 1.00 & & & & & & \\
\hline 45 & BUS & 51 & 1.00 & & & & & & \\
\hline 46 & BUS & 100 & 1.00 & & & & & & \\
\hline 47 & $\div$ & & & & & & & & \\
\hline 48 & $\frac{\circ}{0}$ & from & to & Rse & Xse & Gsh & Bsh & xo & Vis \\
\hline 49 & LINE & 1 & 3 & 0.00313 & 0.05324 & 0.00000 & 0.00000 & 0.00000 & 3 \\
\hline 50 & LINE & 2 & 4 & 0.00313 & 0.05324 & 0.00000 & 0.00000 & 0.00000 & 3 \\
\hline 51 & LINE & 5 & 39 & 0.04314 & 0.34514 & 0.00000 & 0.00000 & 0.00000 & 3 \\
\hline 52 & LINE & 5 & 49 & 0.05918 & 0.35510 & 0.00000 & 0.00000 & 0.00000 & 3 \\
\hline 53 & LINE & 6 & 11 & 0.05575 & 0.36240 & 0.00000 & 0.00000 & 0.00000 & 3 \\
\hline 54 & LINE & 6 & 19 & 0.01218 & 0.14616 & 0.00000 & 0.00000 & 0.00000 & 3 \\
\hline 55 & LINE & 12 & 17 & 0.06843 & 0.44477 & 0.00000 & 0.00000 & 0.00000 & 3 \\
\hline
\end{tabular}




\begin{tabular}{|c|c|c|c|c|c|c|c|c|}
\hline 56 & LINE & 13 & 18 & 0.05829 & 0.37888 & 0.00000 & 0.00000 & 0.00000 \\
\hline 57 & LINE & 15 & 20 & 0.01218 & 0.14616 & 0.00000 & 0.00000 & 0.00000 \\
\hline 58 & LINE & 16 & 21 & 0.15036 & 0.75178 & 0.00000 & 0.00000 & 0.00000 \\
\hline 59 & LINE & 25 & 28 & 0.05829 & 0.37888 & 0.00000 & 0.00000 & 0.00000 \\
\hline 60 & LINE & 26 & 29 & 0.05829 & 0.37888 & 0.00000 & 0.00000 & 0.00000 \\
\hline 61 & LINE & 27 & 30 & 0.05829 & 0.37888 & 0.00000 & 0.00000 & 0.00000 \\
\hline 62 & LINE & 31 & 36 & 0.02289 & 0.22886 & 0.00000 & 0.00000 & 0.00000 \\
\hline 63 & LINE & 32 & 37 & 0.10286 & 0.56573 & 0.00000 & 0.00000 & 0.00000 \\
\hline 64 & LINE & 50 & 51 & 0.06395 & 0.37796 & 0.00000 & 0.00000 & 0.00000 \\
\hline 65 & LINE & 3 & 5 & 0.00075 & 0.00063 & 0.00000 & 0.00000 & 0.00000 \\
\hline 66 & LINE & 3 & 6 & 0.00109 & 0.00091 & 0.00000 & 0.00000 & 0.00000 \\
\hline 67 & LINE & 3 & 9 & 0.00150 & 0.00125 & 0.00000 & 0.00000 & 0.00000 \\
\hline 68 & LINE & 3 & 26 & 0.00157 & 0.00131 & 0.00000 & 0.00000 & 0.00000 \\
\hline 69 & LINE & 4 & 8 & 0.00076 & 0.00092 & 0.00000 & 0.00000 & 0.00000 \\
\hline 70 & LINE & 4 & 15 & 0.00227 & 0.00189 & 0.00000 & 0.00000 & 0.00000 \\
\hline 71 & LINE & 4 & 24 & 0.00118 & 0.00098 & 0.00000 & 0.00000 & 0.00000 \\
\hline 72 & LINE & 4 & 16 & 0.00274 & 0.00229 & 0.00000 & 0.00000 & 0.00000 \\
\hline 73 & LINE & 4 & 27 & 0.00143 & 0.00119 & 0.00000 & 0.00000 & 0.00000 \\
\hline 74 & LINE & 9 & 12 & 0.00038 & 0.00032 & 0.00000 & 0.00000 & 0.00000 \\
\hline 75 & LINE & 9 & 25 & 0.00424 & 0.00353 & 0.00000 & 0.00000 & 0.00000 \\
\hline 76 & LINE & 10 & 13 & 0.00046 & 0.00039 & 0.00000 & 0.00000 & 0.00000 \\
\hline 77 & LINE & 10 & 27 & 0.00110 & 0.00091 & 0.00000 & 0.00000 & 0.00000 \\
\hline 78 & LINE & 17 & 22 & 0.03813 & 0.02451 & 0.00000 & 0.00000 & 0.00000 \\
\hline 79 & LINE & 18 & 23 & 0.03813 & 0.02451 & 0.00000 & 0.00000 & 0.00000 \\
\hline 80 & LINE & 24 & 31 & 0.00079 & 0.00065 & 0.00000 & 0.00000 & 0.00000 \\
\hline 81 & LINE & 24 & 32 & 0.00112 & 0.00093 & 0.00000 & 0.00000 & 0.00000 \\
\hline 82 & LINE & 28 & 33 & 0.03813 & 0.02451 & 0.00000 & 0.00000 & 0.00000 \\
\hline 83 & LINE & 28 & 41 & 0.03429 & 0.02105 & 0.00000 & 0.00000 & 0.00000 \\
\hline 84 & LINE & 29 & 34 & 0.03813 & 0.02451 & 0.00000 & 0.00000 & 0.00000 \\
\hline 85 & LINE & 29 & 38 & 0.08024 & 0.07732 & 0.00000 & 0.00000 & 0.00000 \\
\hline 86 & LINE & 29 & 38 & 0.08024 & 0.07732 & 0.00000 & 0.00000 & 0.00000 \\
\hline 87 & LINE & 30 & 35 & 0.03813 & 0.02451 & 0.00000 & 0.00000 & 0.00000 \\
\hline 88 & LINE & 50 & 3 & 0.00243 & 0.00485 & 0.00000 & 0.00000 & 0.00000 \\
\hline 89 & LINE & 50 & 3 & 0.00243 & 0.00485 & 0.00000 & 0.00000 & 0.00000 \\
\hline 90 & LINE & 100 & 1 & 0.00139 & 0.00296 & 0.00000 & 0.00000 & 0.00000 \\
\hline 91 & LINE & 100 & 2 & 0.00139 & 0.00296 & 0.00000 & 0.00000 & 0.00000 \\
\hline
\end{tabular}

$92 \div$

94 GENERATOR

95 GENERATOR 50

96 GENERATOR 100

$97 \%$

$98 \div$

99 MOTOR

100 MOTOR

101 MOTOR

102 MOTOR

103 MOTOR

104 MOTOR

105 MOTOR

106 MOTOR

107 MOTOR

108 MOTOR

109 MOTOR

110 MOTOR

bus
4
50
100

bus
11
17
17
18
18
19
19
20
20
21
22
23

0.003

0.002

$\mathrm{Xs}$
0.000

$\mathrm{xp}$

0.000

0.000

0.000

0.000

0.000

$\begin{array}{ll}R & X S \\ 0.352 & 0.000 \\ 0.338 & 0.000 \\ 0.802 & 0.000 \\ 0.338 & 0.000 \\ 0.802 & 0.000 \\ 0.057 & 0.000 \\ 0.047 & 0.000 \\ 0.067 & 0.000 \\ 0.060 & 0.000 \\ 0.320 & 0.000 \\ 1.398 & 0.000 \\ 1.398 & 0.000\end{array}$

Xpp
0.102

$\mathrm{x} 2$

xo

0.072

0.000

0.000

0.010

$0.000 \quad 0.000$

$0.000 \quad 0.000$

bus

17

17

18

18

19

19

20

20

21

23 $\mathrm{xp}$

0.000

Xpp

0.000

0.000

0.000

0.000

0.000

0.000

0.000

0.000

0.000

0.000

0.000

4.219

x2

0.000

$\mathrm{x} 0$

3.384

0.000

0.000

4.008

0.000

0.000

3.384

0.000

0.000

4.008

0.000

.000

1.484

0.000

0.000

0.703

0.000

0.000

1.005

0.000

.000

1.556

0.000

0.000

3.835

0.000

0.000

19.571

0.000

0.000

19.571

0.000

0.000 


$\begin{array}{llllllll}111 \text { MOTOR } & 28 & 0.697 & 0.000 & 0.000 & 6.972 & 0.000 & 0.000 \\ 112 \text { MOTOR } & 28 & 0.802 & 0.000 & 0.000 & 4.008 & 0.000 & 0.000 \\ 113 \text { MOTOR } & 29 & 0.321 & 0.000 & 0.000 & 3.206 & 0.000 & 0.000 \\ 114 \text { MOTOR } & 29 & 1.199 & 0.000 & 0.000 & 5.998 & 0.000 & 0.000 \\ 115 \text { MOTOR } & 30 & 0.431 & 0.000 & 0.000 & 5.166 & 0.000 & 0.000 \\ 116 \text { MOTOR } & 30 & 1.116 & 0.000 & 0.000 & 5.578 & 0.000 & 0.000 \\ 117 \text { MOTOR } & 33 & 0.809 & 0.000 & 0.000 & 9.701 & 0.000 & 0.000 \\ 118 \text { MOTOR } & 34 & 3.621 & 0.000 & 0.000 & 25.354 & 0.000 & 0.000 \\ 119 \text { MOTOR } & 35 & 0.809 & 0.000 & 0.000 & 9.701 & 0.000 & 0.000 \\ 120 \text { MOTOR } & 36 & 0.025 & 0.000 & 0.000 & 0.818 & 0.000 & 0.000 \\ 121 \text { MOTOR } & 37 & 0.246 & 0.000 & 0.000 & 2.952 & 0.000 & 0.000 \\ 122 \text { MOTOR } & 37 & 1.859 & 0.000 & 0.000 & 9.296 & 0.000 & 0.000 \\ 123 \text { MOTOR } & 39 & 0.034 & 0.000 & 0.000 & 1.005 & 0.000 & 0.000 \\ 124 \text { MOTOR } & 49 & 0.264 & 0.000 & 0.000 & 2.640 & 0.000 & 0.000 \\ 125 \text { MOTOR } & 51 & 1.432 & 0.000 & 0.000 & 10.020 & 0.000 & 0.000 \\ 126 \text { MOTOR } & 51 & 0.408 & 0.000 & 0.000 & 4.893 & 0.000 & 0.000 \\ 127 \text { MOTOR } & 8 & 0.006 & 0.000 & 0.000 & 0.222 & 0.000 & 0.000\end{array}$

$128 \%$

129 응

bus

130 FAULT

4

Calc Type $3 \mathrm{P}$

Calc_time

$(0=a 11 ; 1=$ sub; $2=\operatorname{trans} ; 3=$ ss $)$ 


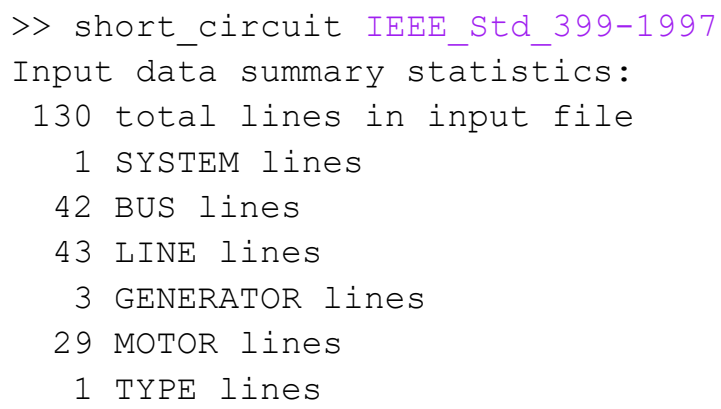

Results for System Name "IEEE_Std_399-1997"

Symmetrical Three-Phase Fault at Bus 4

Calculating Subtransient Currents

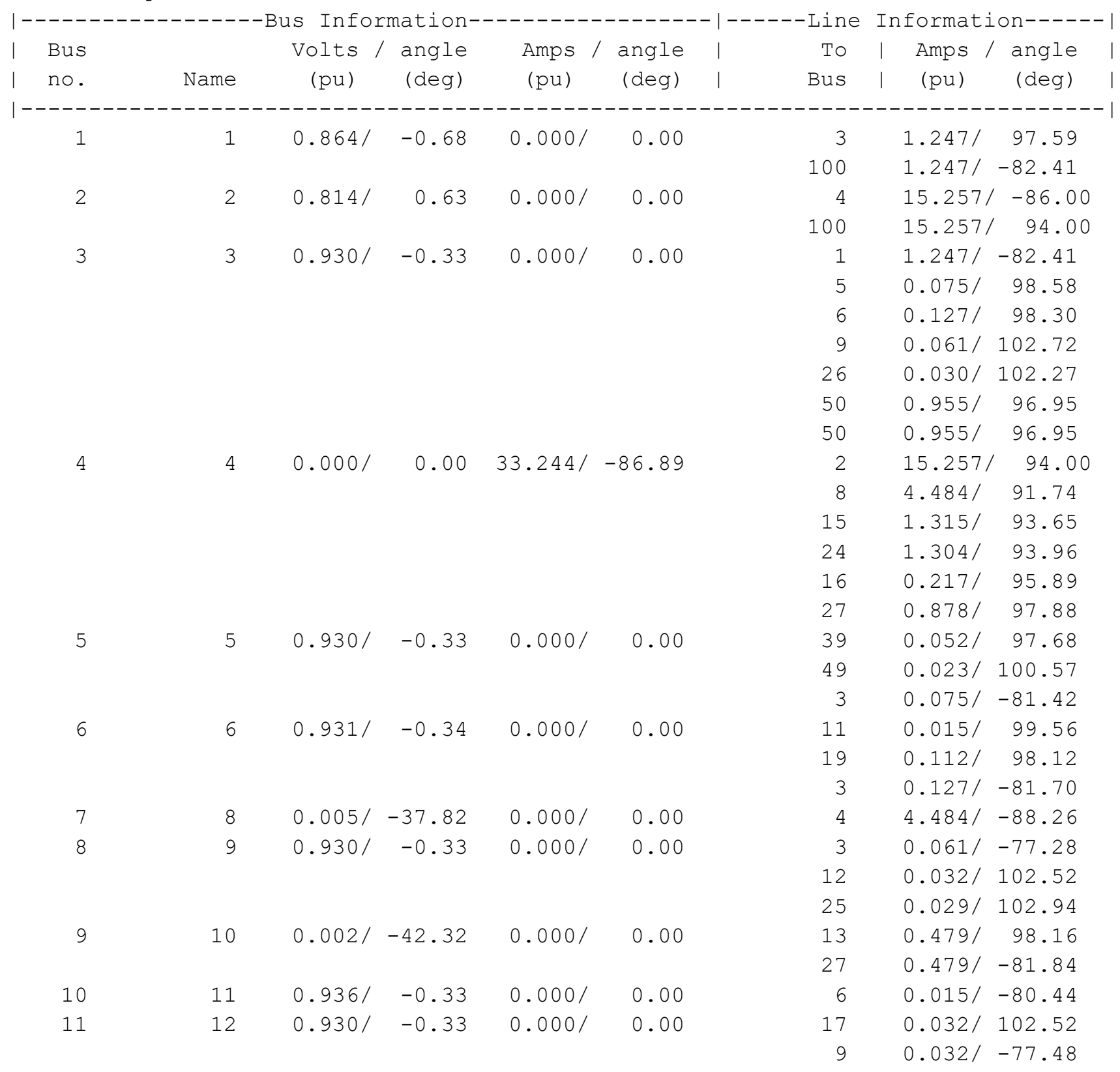




\begin{tabular}{|c|c|c|c|c|c|c|c|c|}
\hline \multirow{2}{*}{12} & \multirow[t]{2}{*}{13} & \multirow[t]{2}{*}{$0.003 /$} & \multirow[t]{2}{*}{-42.24} & \multirow[t]{2}{*}{$0.000 /$} & \multirow[t]{2}{*}{0.00} & 18 & $0.479 /$ & 98.16 \\
\hline & & & & & & 10 & $0.479 /$ & -81.84 \\
\hline \multirow[t]{2}{*}{13} & \multirow[t]{2}{*}{15} & \multirow[t]{2}{*}{$0.004 /$} & \multirow[t]{2}{*}{-46.57} & \multirow[t]{2}{*}{$0.000 /$} & \multirow[t]{2}{*}{0.00} & 20 & $1.315 /$ & 93.65 \\
\hline & & & & & & 4 & $1.315 /$ & -86.35 \\
\hline \multirow[t]{2}{*}{14} & \multirow[t]{2}{*}{16} & \multirow[t]{2}{*}{$0.001 /$} & \multirow[t]{2}{*}{-44.23} & \multirow[t]{2}{*}{$0.000 /$} & \multirow[t]{2}{*}{0.00} & 21 & $0.217 /$ & 95.89 \\
\hline & & & & & & 4 & $0.217 /$ & -84.11 \\
\hline \multirow[t]{2}{*}{15} & \multirow[t]{2}{*}{17} & $0.945 /$ & -0.27 & $0.000 /$ & 0.00 & 12 & $0.032 /$ & -77.48 \\
\hline & & & & & & 22 & $0.003 /$ & 98.81 \\
\hline 16 & 18 & $0.186 /$ & -1.12 & $0.000 /$ & 0.00 & 13 & $0.479 /$ & -81.84 \\
\hline & & & & & & 23 & $0.041 /$ & 94.45 \\
\hline 17 & 19 & $0.947 /$ & -0.27 & $0.000 /$ & 0.00 & 6 & $0.112 /$ & -81.88 \\
\hline 18 & 20 & $0.196 /$ & -1.92 & $0.000 /$ & 0.00 & 15 & $1.315 /$ & -86.35 \\
\hline 19 & 21 & $0.167 /$ & -5.59 & $0.000 /$ & 0.00 & 16 & $0.217 /$ & -84.11 \\
\hline 20 & 22 & $0.945 /$ & -0.28 & $0.000 /$ & 0.00 & 17 & $0.003 /$ & -81.19 \\
\hline 21 & 23 & $0.187 /$ & -1.57 & $0.000 /$ & 0.00 & 18 & $0.041 /$ & -85.55 \\
\hline 22 & 24 & $0.002 /$ & -46.33 & $0.000 /$ & 0.00 & 4 & $1.304 /$ & -86.04 \\
\hline & & & & & & 31 & $0.952 /$ & 92.74 \\
\hline & & & & & & 32 & $0.352 /$ & 97.23 \\
\hline 23 & 25 & $0.931 /$ & -0.34 & $0.000 /$ & 0.00 & 28 & $0.029 /$ & 102.94 \\
\hline & & & & & & 9 & $0.029 /$ & -77.06 \\
\hline 24 & 26 & $0.930 /$ & -0.33 & $0.000 /$ & 0.00 & 29 & $0.030 /$ & 102.27 \\
\hline & & & & & & 3 & $0.030 /$ & -77.73 \\
\hline 25 & 27 & $0.002 /$ & -42.35 & $0.000 /$ & 0.00 & 30 & $0.398 /$ & 97.55 \\
\hline & & & & & & 4 & $0.878 /$ & -82.12 \\
\hline & & & & & & 10 & $0.479 /$ & 98.16 \\
\hline 26 & 28 & $0.942 /$ & -0.29 & $0.000 /$ & 0.00 & 25 & $0.029 /$ & -77.06 \\
\hline & & & & & & 33 & $0.006 /$ & 99.56 \\
\hline & & & & & & 41 & $0.000 /$ & 149.80 \\
\hline 27 & 29 & $0.942 /$ & -0.28 & $0.000 /$ & 0.00 & 26 & $0.030 /$ & -77.73 \\
\hline & & & & & & 34 & $0.002 /$ & 102.78 \\
\hline & & & & & & 38 & $0.000 /$ & 139.19 \\
\hline & & & & & & 38 & $0.000 /$ & 139.19 \\
\hline 28 & 30 & $0.154 /$ & $-1 \cdot 60$ & $0.000 /$ & 0.00 & 27 & $0.398 /$ & -82.45 \\
\hline & & & & & & 35 & $0.087 /$ & 95.27 \\
\hline 29 & 31 & $0.003 /$ & -46.82 & $0.000 /$ & 0.00 & 36 & $0.952 /$ & 92.74 \\
\hline & & & & & & 24 & $0.952 /$ & -87.26 \\
\hline 30 & 32 & $0.003 /$ & -45.67 & $0.000 /$ & 0.00 & 37 & $0.352 /$ & 97.23 \\
\hline & & & & & & 24 & $0.352 /$ & -82.77 \\
\hline 31 & 33 & $0.942 /$ & -0.30 & $0.000 /$ & 0.00 & 28 & $0.006 /$ & -80.44 \\
\hline 32 & 34 & $0.942 /$ & -0.29 & $0.000 /$ & 0.00 & 29 & $0.002 /$ & -77.22 \\
\hline 33 & 35 & $0.156 /$ & -2.71 & $0.000 /$ & 0.00 & 30 & $0.087 /$ & -84.73 \\
\hline 34 & 36 & $0.221 /$ & -3.50 & $0.000 /$ & 0.00 & 31 & $0.952 /$ & -87.26 \\
\hline 35 & 37 & $0.204 /$ & -3.55 & $0.000 /$ & 0.00 & 32 & $0.352 /$ & -82.77 \\
\hline 36 & 38 & $0.942 /$ & -0.28 & $0.000 /$ & 0.00 & 29 & $0.000 /$ & -40.81 \\
\hline & & & & & & 29 & $0.000 /$ & -40.81 \\
\hline 37 & 39 & $0.948 /$ & -0.31 & $0.000 /$ & 0.00 & 5 & $0.052 /$ & -82.32 \\
\hline 38 & 41 & $0.942 /$ & -0.29 & $0.000 /$ & 0.00 & 28 & $0.000 /$ & -30.20 \\
\hline 39 & 49 & $0.939 /$ & -0.32 & $0.000 /$ & 0.00 & 5 & $0.023 /$ & -79.43 \\
\hline & 50 & $0.933 /$ & -0.38 & $0.000 /$ & 0.00 & 51 & $0.018 /$ & 101.52 \\
\hline & & & & & & 3 & $0.955 /$ & -83.05 \\
\hline
\end{tabular}




$\begin{array}{rrrrrr}41 & 51 & 0.940 / & -0.36 & 0.000 / & 0.00 \\ 42 & 100 & 0.860 / & -0.60 & 0.000 / & 0.00\end{array}$

$\begin{array}{lll}3 & 0.955 / & -83.05 \\ 0 & 0.018 / & -78.48 \\ 1 & 1.247 / & 97.59 \\ 2 & 15.257 / & -86.00\end{array}$

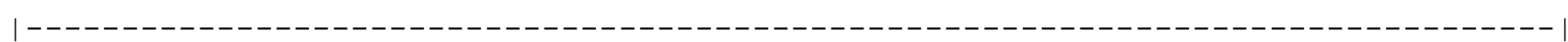




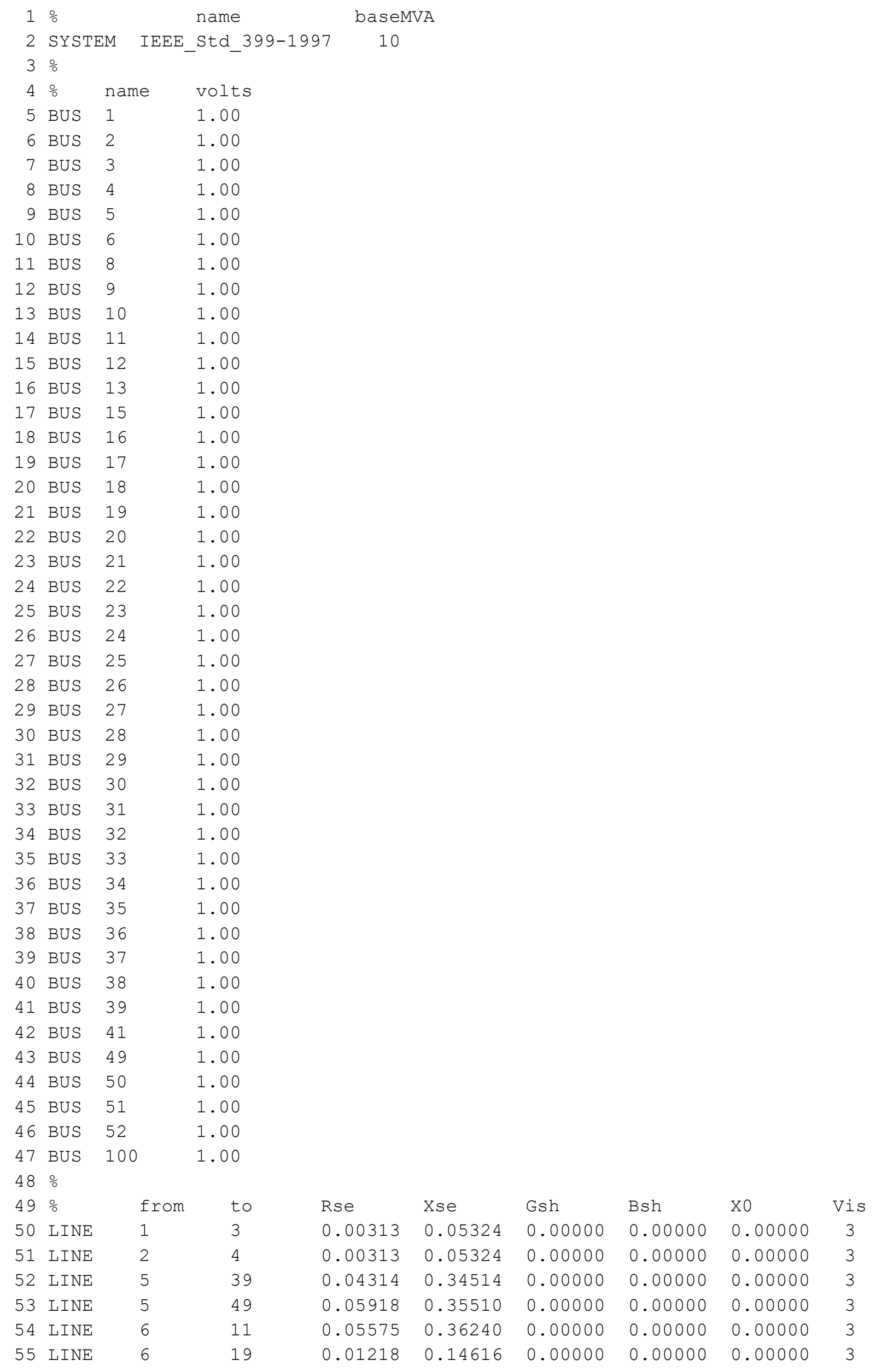




\begin{tabular}{|c|c|c|c|c|c|c|c|c|}
\hline 56 & LINE & 12 & 17 & 0.06843 & 0.44477 & 0.00000 & 0.00000 & 0.00000 \\
\hline 57 & LINE & 13 & 18 & 0.05829 & 0.37888 & 0.00000 & 0.00000 & 0.00000 \\
\hline 58 & LINE & 15 & 20 & 0.01218 & 0.14616 & 0.00000 & 0.00000 & 0.00000 \\
\hline 59 & LINE & 16 & 21 & 0.15036 & 0.75178 & 0.00000 & 0.00000 & 0.00000 \\
\hline 60 & LINE & 25 & 28 & 0.05829 & 0.37888 & 0.00000 & 0.00000 & 0.00000 \\
\hline 61 & LINE & 26 & 29 & 0.05829 & 0.37888 & 0.00000 & 0.00000 & 0.00000 \\
\hline 62 & LINE & 27 & 30 & 0.05829 & 0.37888 & 0.00000 & 0.00000 & 0.00000 \\
\hline 63 & LINE & 31 & 36 & 0.02289 & 0.22886 & 0.00000 & 0.00000 & 0.00000 \\
\hline 64 & LINE & 32 & 37 & 0.10286 & 0.56573 & 0.00000 & 0.00000 & 0.00000 \\
\hline 65 & LINE & 50 & 51 & 0.06395 & 0.37796 & 0.00000 & 0.00000 & 0.00000 \\
\hline 66 & LINE & 52 & 4 & 0.00313 & 0.05324 & 0.00000 & 0.00000 & 0.00000 \\
\hline 67 & LINE & 3 & 5 & 0.00075 & 0.00063 & 0.00000 & 0.00000 & 0.00000 \\
\hline 68 & LINE & 3 & 6 & 0.00109 & 0.00091 & 0.00000 & 0.00000 & 0.00000 \\
\hline 69 & LINE & 3 & 9 & 0.00150 & 0.00125 & 0.00000 & 0.00000 & 0.00000 \\
\hline 70 & LINE & 3 & 26 & 0.00157 & 0.00131 & 0.00000 & 0.00000 & 0.00000 \\
\hline 71 & LINE & 4 & 8 & 0.00076 & 0.00092 & 0.00000 & 0.00000 & 0.00000 \\
\hline 72 & LINE & 4 & 15 & 0.00227 & 0.00189 & 0.00000 & 0.00000 & 0.00000 \\
\hline 73 & LINE & 4 & 24 & 0.00118 & 0.00098 & 0.00000 & 0.00000 & 0.00000 \\
\hline 74 & LINE & 4 & 16 & 0.00274 & 0.00229 & 0.00000 & 0.00000 & 0.00000 \\
\hline 75 & LINE & 4 & 27 & 0.00143 & 0.00119 & 0.00000 & 0.00000 & 0.00000 \\
\hline 76 & LINE & 9 & 12 & 0.00038 & 0.00032 & 0.00000 & 0.00000 & 0.00000 \\
\hline 77 & LINE & 9 & 25 & 0.00424 & 0.00353 & 0.00000 & 0.00000 & 0.00000 \\
\hline 78 & LINE & 10 & 13 & 0.00046 & 0.00039 & 0.00000 & 0.00000 & 0.00000 \\
\hline 79 & LINE & 10 & 27 & 0.00110 & 0.00091 & 0.00000 & 0.00000 & 0.00000 \\
\hline 80 & LINE & 17 & 22 & 0.03813 & 0.02451 & 0.00000 & 0.00000 & 0.00000 \\
\hline 81 & LINE & 18 & 23 & 0.03813 & 0.02451 & 0.00000 & 0.00000 & 0.00000 \\
\hline 82 & LINE & 24 & 31 & 0.00079 & 0.00065 & 0.00000 & 0.00000 & 0.00000 \\
\hline 83 & LINE & 24 & 32 & 0.00112 & 0.00093 & 0.00000 & 0.00000 & 0.00000 \\
\hline 84 & LINE & 28 & 33 & 0.03813 & 0.02451 & 0.00000 & 0.00000 & 0.00000 \\
\hline 85 & LINE & 28 & 41 & 0.03429 & 0.02105 & 0.00000 & 0.00000 & 0.00000 \\
\hline 86 & LINE & 29 & 34 & 0.03813 & 0.02451 & 0.00000 & 0.00000 & 0.00000 \\
\hline 87 & LINE & 29 & 38 & 0.08024 & 0.07732 & 0.00000 & 0.00000 & 0.00000 \\
\hline 88 & LINE & 29 & 38 & 0.08024 & 0.07732 & 0.00000 & 0.00000 & 0.00000 \\
\hline 89 & LINE & 30 & 35 & 0.03813 & 0.02451 & 0.00000 & 0.00000 & 0.00000 \\
\hline 90 & LINE & 50 & 3 & 0.00243 & 0.00485 & 0.00000 & 0.00000 & 0.00000 \\
\hline 91 & LINE & 50 & 3 & 0.00243 & 0.00485 & 0.00000 & 0.00000 & 0.00000 \\
\hline 92 & LINE & 100 & 1 & 0.00139 & 0.00296 & 0.00000 & 0.00000 & 0.00000 \\
\hline 93 & LINE & 100 & 2 & 0.00139 & 0.00296 & 0.00000 & 0.00000 & 0.00000 \\
\hline
\end{tabular}

$94 \div$

\begin{tabular}{|c|c|}
\hline $5 \%$ & bus \\
\hline 6 GENERATOR & 50 \\
\hline GENERATOR & 52 \\
\hline 3 GENERATOR & 100 \\
\hline
\end{tabular}

$100 \%$

101 MOTOR

bus

102 MOTOR

103 MOTOR

104 MOTOR

105 MOTOR

106 MOTOR

107 MOTOR

108 MOTOR

109 MOTOR

110 MOTOR

0.002

0.000

0.000

$\mathrm{Xs}$

0.000

0.000

0.000

\section{$\mathrm{xp}$}

0.000

0.000

0.000

$\begin{array}{ll}R & X s \\ 0.352 & 0.000 \\ 0.338 & 0.000 \\ 0.802 & 0.000 \\ 0.338 & 0.000 \\ 0.802 & 0.000 \\ 0.057 & 0.000 \\ 0.047 & 0.000 \\ 0.067 & 0.000 \\ 0.060 & 0.000 \\ 0.320 & 0.000\end{array}$

$\mathrm{xp}$

0.000

0.000

0.000

0.000

0.000

0.000

$\mathrm{Xpp}$

0.072

0.347

0.010

\section{$\mathrm{x} 2$}

0.000

0.000

0.000

$\mathrm{x} 0$

0.000

0.000

0.000

Xpp $\quad$ X2 20

4.219

0.000

0.000

3.384

0.000

0.000

4.008

0.000

0.000

3.384

0.000

0.000

4.008

0.000

0.000

1.484

0.000

0.000

$0.000 \quad 0.703$

0.000

0.000

$0.000 \quad 1.005$

0.000

0.000

0.0001 .556

0.000

0.000

$0.000 \quad 3.835$

0.000

0.000 


\begin{tabular}{|c|c|c|c|c|c|c|c|c|c|}
\hline 111 & MOTOR & & 22 & 1.398 & 0.000 & 0.000 & 19.571 & 0.000 & 0.000 \\
\hline 112 & MOTOR & & 23 & 1.398 & 0.000 & 0.000 & 19.571 & 0.000 & 0.000 \\
\hline 113 & MOTOR & & 28 & 0.697 & 0.000 & 0.000 & 6.972 & 0.000 & 0.000 \\
\hline 114 & MOTOR & & 28 & 0.802 & 0.000 & 0.000 & 4.008 & 0.000 & 0.000 \\
\hline 115 & MOTOR & & 29 & 0.321 & 0.000 & 0.000 & 3.206 & 0.000 & 0.000 \\
\hline 116 & MOTOR & & 29 & 1.199 & 0.000 & 0.000 & 5.998 & 0.000 & 0.000 \\
\hline 117 & MOTOR & & 30 & 0.431 & 0.000 & 0.000 & 5.166 & 0.000 & 0.000 \\
\hline 118 & MOTOR & & 30 & 1.116 & 0.000 & 0.000 & 5.578 & 0.000 & 0.000 \\
\hline 119 & MOTOR & & 33 & 0.809 & 0.000 & 0.000 & 9.701 & 0.000 & 0.000 \\
\hline 120 & MOTOR & & 34 & 3.621 & 0.000 & 0.000 & 25.354 & 0.000 & 0.000 \\
\hline 121 & MOTOR & & 35 & 0.809 & 0.000 & 0.000 & 9.701 & 0.000 & 0.000 \\
\hline 122 & MOTOR & & 36 & 0.025 & 0.000 & 0.000 & 0.818 & 0.000 & 0.000 \\
\hline 123 & MOTOR & & 37 & 0.246 & 0.000 & 0.000 & 2.952 & 0.000 & 0.000 \\
\hline 124 & MOTOR & & 37 & 1.859 & 0.000 & 0.000 & 9.296 & 0.000 & 0.000 \\
\hline 125 & MOTOR & & 39 & 0.034 & 0.000 & 0.000 & 1.005 & 0.000 & 0.000 \\
\hline 126 & MOTOR & & 49 & 0.264 & 0.000 & 0.000 & 2.640 & 0.000 & 0.000 \\
\hline 127 & MOTOR & & 51 & 1.432 & 0.000 & 0.000 & 10.020 & 0.000 & 0.000 \\
\hline 128 & MOTOR & & 51 & 0.408 & 0.000 & 0.000 & 4.893 & 0.000 & 0.000 \\
\hline 129 & MOTOR & & 8 & 0.006 & 0.000 & 0.000 & 0.222 & 0.000 & 0.000 \\
\hline 130 & $\%$ & & & & & & & & \\
\hline 131 & \% & bus & Calc & Type & Lc_time & $(\Omega-A]$ & $1-\mathrm{S}$ ibt & nsien & ;2-Transient;3-Steady-state \\
\hline 132 & FAULT & 20 & 3 & & $\overline{1}$ & & & & \\
\hline
\end{tabular}




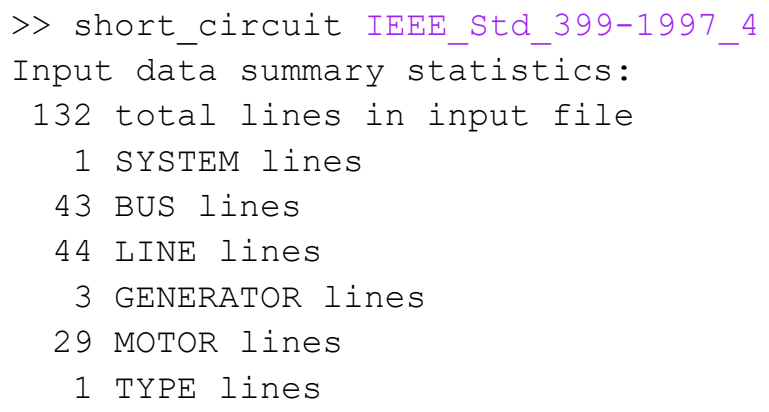

Results for System Name "IEEE_Std_399-1997"

Symmetrical Three-Phase Fault at Bus 20

Calculating Subtransient Currents

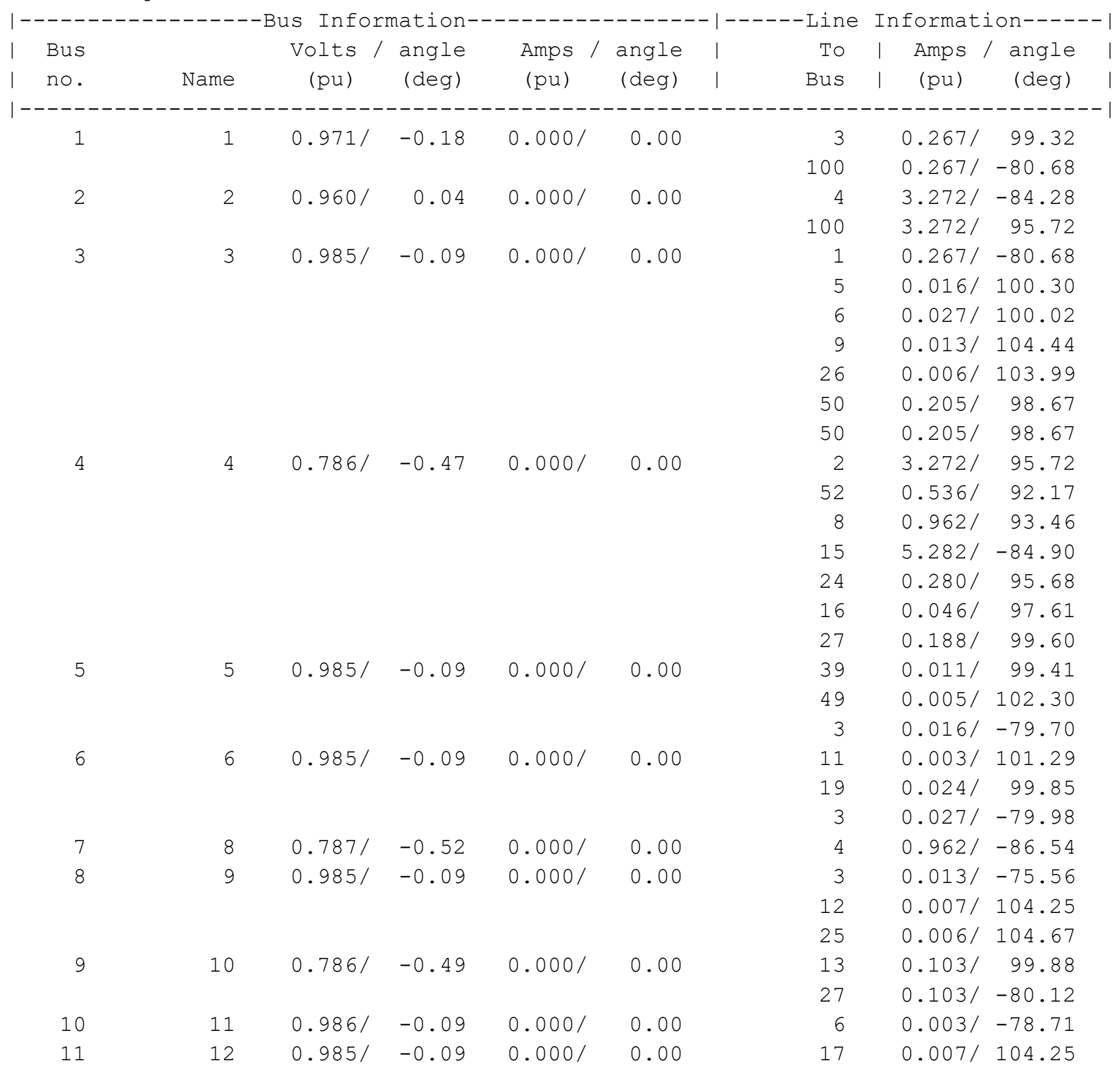




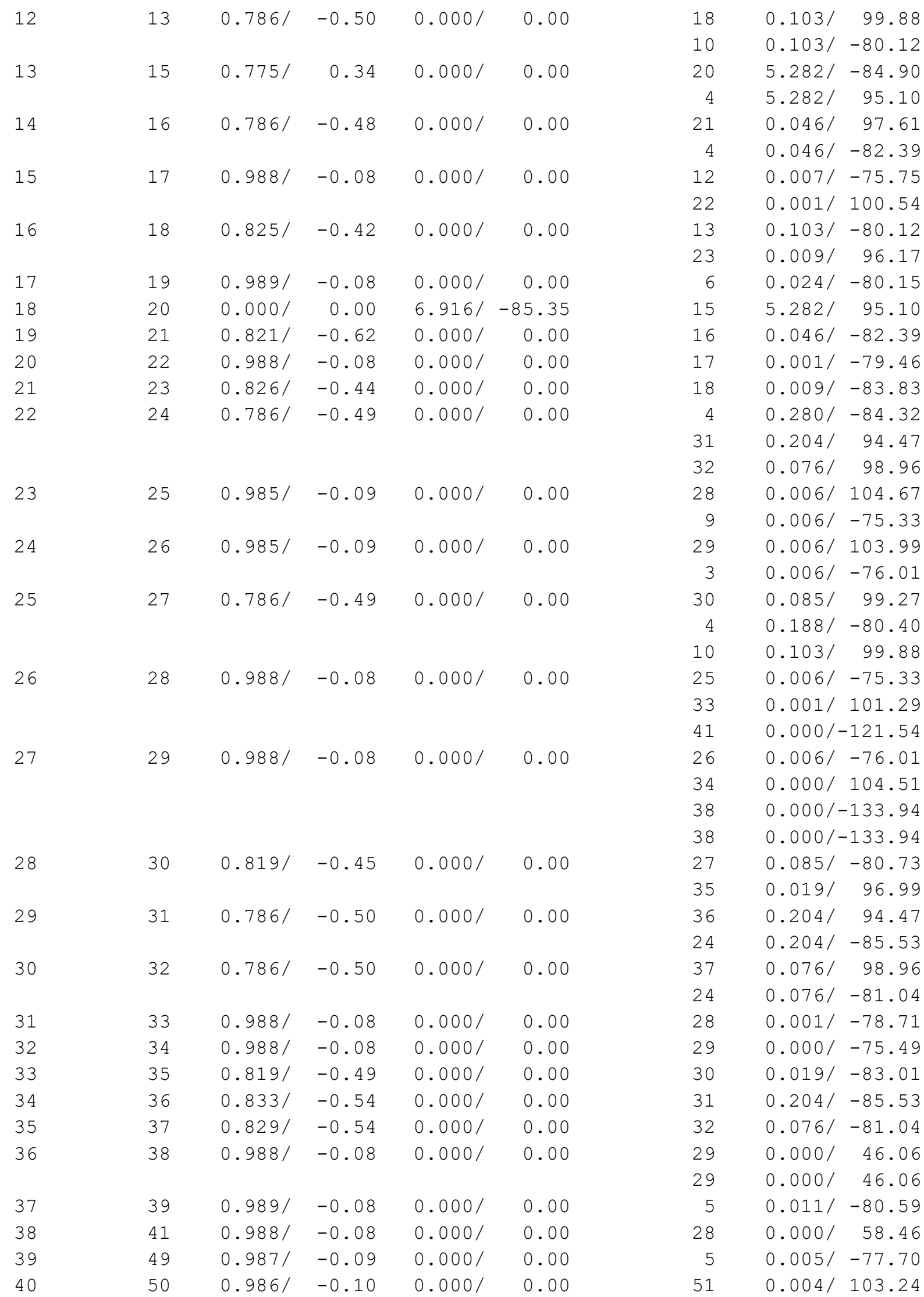




$\begin{array}{lrllll}41 & 51 & 0.987 / & -0.10 & 0.000 / & 0.00 \\ 42 & 52 & 0.814 / & -0.50 & 0.000 / & 0.00 \\ 43 & 100 & 0.970 / & -0.17 & 0.000 / & 0.00\end{array}$

$30.205 /-81.33$

$30.205 /-81.33$

41

43

$0.000 /$

50

$0.004 /-76.76$

$40.536 /-87.83$

$10.267 / 99.32$

$2 \quad 3.272 /-84.28$

$>>$ 


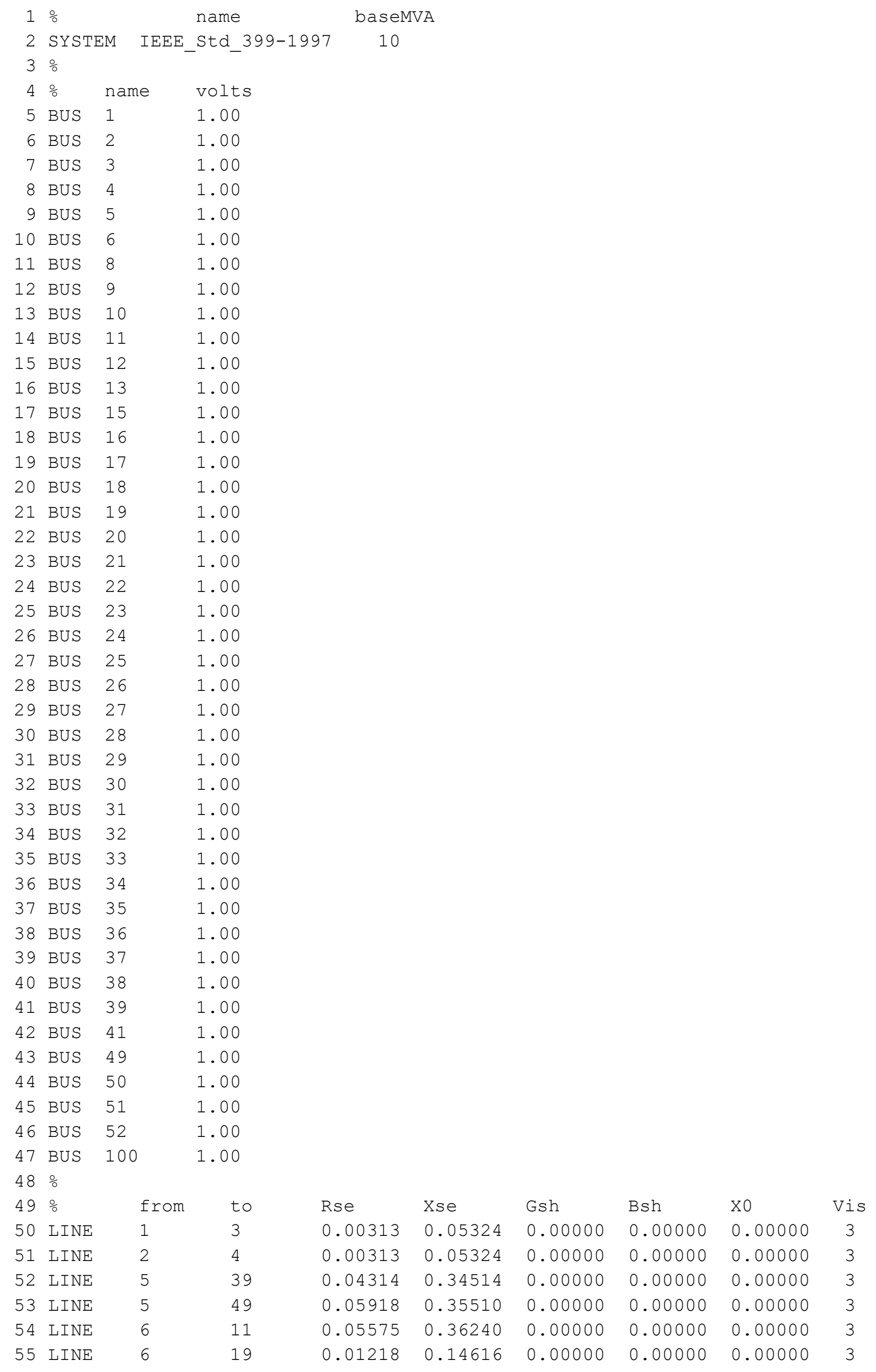




\begin{tabular}{|c|c|c|c|c|c|c|c|c|}
\hline 56 & LINE & 12 & 17 & 0.06843 & 0.44477 & 0.00000 & 0.00000 & 0.00000 \\
\hline 57 & LINE & 13 & 18 & 0.05829 & 0.37888 & 0.00000 & 0.00000 & 0.00000 \\
\hline 58 & LINE & 15 & 20 & 0.01218 & 0.14616 & 0.00000 & 0.00000 & 0.00000 \\
\hline 59 & LINE & 16 & 21 & 0.15036 & 0.75178 & 0.00000 & 0.00000 & 0.00000 \\
\hline 60 & LINE & 25 & 28 & 0.05829 & 0.37888 & 0.00000 & 0.00000 & 0.00000 \\
\hline 61 & LINE & 26 & 29 & 0.05829 & 0.37888 & 0.00000 & 0.00000 & 0.00000 \\
\hline 62 & LINE & 27 & 30 & 0.05829 & 0.37888 & 0.00000 & 0.00000 & 0.00000 \\
\hline 63 & LINE & 31 & 36 & 0.02289 & 0.22886 & 0.00000 & 0.00000 & 0.00000 \\
\hline 64 & LINE & 32 & 37 & 0.10286 & 0.56573 & 0.00000 & 0.00000 & 0.00000 \\
\hline 65 & LINE & 50 & 51 & 0.06395 & 0.37796 & 0.00000 & 0.00000 & 0.00000 \\
\hline 66 & LINE & 52 & 4 & 0.00313 & 0.05324 & 0.00000 & 0.00000 & 0.00000 \\
\hline 67 & LINE & 3 & 5 & 0.00075 & 0.00063 & 0.00000 & 0.00000 & 0.00000 \\
\hline 68 & LINE & 3 & 6 & 0.00109 & 0.00091 & 0.00000 & 0.00000 & 0.00000 \\
\hline 69 & LINE & 3 & 9 & 0.00150 & 0.00125 & 0.00000 & 0.00000 & 0.00000 \\
\hline 70 & LINE & 3 & 26 & 0.00157 & 0.00131 & 0.00000 & 0.00000 & 0.00000 \\
\hline 71 & LINE & 4 & 8 & 0.00076 & 0.00092 & 0.00000 & 0.00000 & 0.00000 \\
\hline 72 & LINE & 4 & 15 & 0.00227 & 0.00189 & 0.00000 & 0.00000 & 0.00000 \\
\hline 73 & LINE & 4 & 24 & 0.00118 & 0.00098 & 0.00000 & 0.00000 & 0.00000 \\
\hline 74 & LINE & 4 & 16 & 0.00274 & 0.00229 & 0.00000 & 0.00000 & 0.00000 \\
\hline 75 & LINE & 4 & 27 & 0.00143 & 0.00119 & 0.00000 & 0.00000 & 0.00000 \\
\hline 76 & LINE & 9 & 12 & 0.00038 & 0.00032 & 0.00000 & 0.00000 & 0.00000 \\
\hline 77 & LINE & 9 & 25 & 0.00424 & 0.00353 & 0.00000 & 0.00000 & 0.00000 \\
\hline 78 & LINE & 10 & 13 & 0.00046 & 0.00039 & 0.00000 & 0.00000 & 0.00000 \\
\hline 79 & LINE & 10 & 27 & 0.00110 & 0.00091 & 0.00000 & 0.00000 & 0.00000 \\
\hline 80 & LINE & 17 & 22 & 0.03813 & 0.02451 & 0.00000 & 0.00000 & 0.00000 \\
\hline 81 & LINE & 18 & 23 & 0.03813 & 0.02451 & 0.00000 & 0.00000 & 0.00000 \\
\hline 82 & LINE & 24 & 31 & 0.00079 & 0.00065 & 0.00000 & 0.00000 & 0.00000 \\
\hline 83 & LINE & 24 & 32 & 0.00112 & 0.00093 & 0.00000 & 0.00000 & 0.00000 \\
\hline 84 & LINE & 28 & 33 & 0.03813 & 0.02451 & 0.00000 & 0.00000 & 0.00000 \\
\hline 85 & LINE & 28 & 41 & 0.03429 & 0.02105 & 0.00000 & 0.00000 & 0.00000 \\
\hline 86 & LINE & 29 & 34 & 0.03813 & 0.02451 & 0.00000 & 0.00000 & 0.00000 \\
\hline 87 & LINE & 29 & 38 & 0.08024 & 0.07732 & 0.00000 & 0.00000 & 0.00000 \\
\hline 88 & LINE & 29 & 38 & 0.08024 & 0.07732 & 0.00000 & 0.00000 & 0.00000 \\
\hline 89 & LINE & 30 & 35 & 0.03813 & 0.02451 & 0.00000 & 0.00000 & 0.00000 \\
\hline 90 & LINE & 50 & 3 & 0.00243 & 0.00485 & 0.00000 & 0.00000 & 0.00000 \\
\hline 91 & LINE & 50 & 3 & 0.00243 & 0.00485 & 0.00000 & 0.00000 & 0.00000 \\
\hline 92 & LINE & 100 & 1 & 0.00139 & 0.00296 & 0.00000 & 0.00000 & 0.00000 \\
\hline 93 & LINE & 100 & 2 & 0.00139 & 0.00296 & 0.00000 & 0.00000 & 0.00000 \\
\hline
\end{tabular}

\begin{tabular}{|c|c|c|c|c|c|c|c|}
\hline $95 \div$ & bus & $\mathrm{R}$ & Xs & $\mathrm{Xp}$ & Xpp & $\mathrm{x} 2$ & xo \\
\hline 96 GENERATOR & 50 & 0.002 & 0.000 & 0.000 & 0.072 & 0.000 & 0.000 \\
\hline 97 GENERATOR & 52 & 0.000 & 0.000 & 0.000 & 0.0325 & 0.000 & 0.000 \\
\hline 98 GENERATOR & 100 & 0.000 & 0.000 & 0.000 & 0.010 & 0.000 & 0.000 \\
\hline $99 \div$ & & & & & & & \\
\hline $100 \%$ & bus & $\mathrm{R}$ & $X s$ & $\mathrm{xp}$ & Xpp & $\mathrm{x} 2$ & xo \\
\hline 101 MOTOR & 11 & 0.352 & 0.000 & 0.000 & 4.219 & 0.000 & 0.000 \\
\hline 102 MOTOR & 17 & 0.338 & 0.000 & 0.000 & 3.384 & 0.000 & 0.000 \\
\hline 103 MOTOR & 17 & 0.802 & 0.000 & 0.000 & 4.008 & 0.000 & 0.000 \\
\hline 104 MOTOR & 18 & 0.338 & 0.000 & 0.000 & 3.384 & 0.000 & 0.000 \\
\hline 105 MOTOR & 18 & 0.802 & 0.000 & 0.000 & 4.008 & 0.000 & 0.000 \\
\hline 106 MOTOR & 19 & 0.057 & 0.000 & 0.000 & 1.484 & 0.000 & 0.000 \\
\hline 107 MOTOR & 19 & 0.047 & 0.000 & 0.000 & 0.703 & 0.000 & 0.000 \\
\hline 108 MOTOR & 20 & 0.067 & 0.000 & 0.000 & 1.005 & 0.000 & 0.000 \\
\hline 109 MOTOR & 20 & 0.060 & 0.000 & 0.000 & 1.556 & 0.000 & 0.000 \\
\hline MOTOR & 21 & 0.320 & 0.000 & 0.000 & 3.835 & 0.000 & 0.000 \\
\hline
\end{tabular}




\begin{tabular}{|c|c|c|c|c|c|c|c|c|c|}
\hline 111 & MOTOR & & 22 & 1.398 & 0.000 & 0.000 & 19.571 & 0.000 & 0.000 \\
\hline 112 & MOTOR & & 23 & 1.398 & 0.000 & 0.000 & 19.571 & 0.000 & 0.000 \\
\hline 113 & MOTOR & & 28 & 0.697 & 0.000 & 0.000 & 6.972 & 0.000 & 0.000 \\
\hline 114 & MOTOR & & 28 & 0.802 & 0.000 & 0.000 & 4.008 & 0.000 & 0.000 \\
\hline 115 & MOTOR & & 29 & 0.321 & 0.000 & 0.000 & 3.206 & 0.000 & 0.000 \\
\hline 116 & MOTOR & & 29 & 1.199 & 0.000 & 0.000 & 5.998 & 0.000 & 0.000 \\
\hline 117 & MOTOR & & 30 & 0.431 & 0.000 & 0.000 & 5.166 & 0.000 & 0.000 \\
\hline 118 & MOTOR & & 30 & 1.116 & 0.000 & 0.000 & 5.578 & 0.000 & 0.000 \\
\hline 119 & MOTOR & & 33 & 0.809 & 0.000 & 0.000 & 9.701 & 0.000 & 0.000 \\
\hline 120 & MOTOR & & 34 & 3.621 & 0.000 & 0.000 & 25.354 & 0.000 & 0.000 \\
\hline 121 & MOTOR & & 35 & 0.809 & 0.000 & 0.000 & 9.701 & 0.000 & 0.000 \\
\hline 122 & MOTOR & & 36 & 0.025 & 0.000 & 0.000 & 0.818 & 0.000 & 0.000 \\
\hline 123 & MOTOR & & 37 & 0.246 & 0.000 & 0.000 & 2.952 & 0.000 & 0.000 \\
\hline 124 & MOTOR & & 37 & 1.859 & 0.000 & 0.000 & 9.296 & 0.000 & 0.000 \\
\hline 125 & MOTOR & & 39 & 0.034 & 0.000 & 0.000 & 1.005 & 0.000 & 0.000 \\
\hline 126 & MOTOR & & 49 & 0.264 & 0.000 & 0.000 & 2.640 & 0.000 & 0.000 \\
\hline 127 & MOTOR & & 51 & 1.432 & 0.000 & 0.000 & 10.020 & 0.000 & 0.000 \\
\hline 128 & MOTOR & & 51 & 0.408 & 0.000 & 0.000 & 4.893 & 0.000 & 0.000 \\
\hline 129 & MOTOR & & 8 & 0.006 & 0.000 & 0.000 & 0.222 & 0.000 & 0.000 \\
\hline 130 & $\%$ & & & & & & & & \\
\hline 131 & \% & bus & Calc & Type & Lc_time & $(\Omega-A]$ & $1-\mathrm{S}$ ibt & nsien & ;2-Transient;3-Steady-state \\
\hline 132 & FAULT & 20 & 3 & & $\overline{1}$ & & & & \\
\hline
\end{tabular}




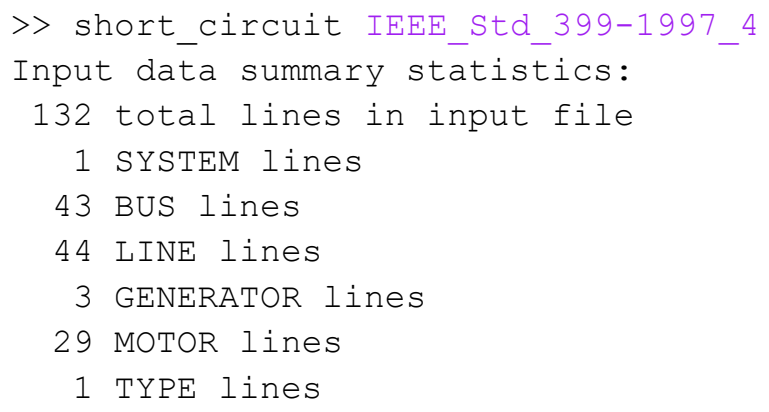

Results for System Name "IEEE_Std_399-1997"

Symmetrical Three-Phase Fault at Bus 20

Calculating Subtransient Currents

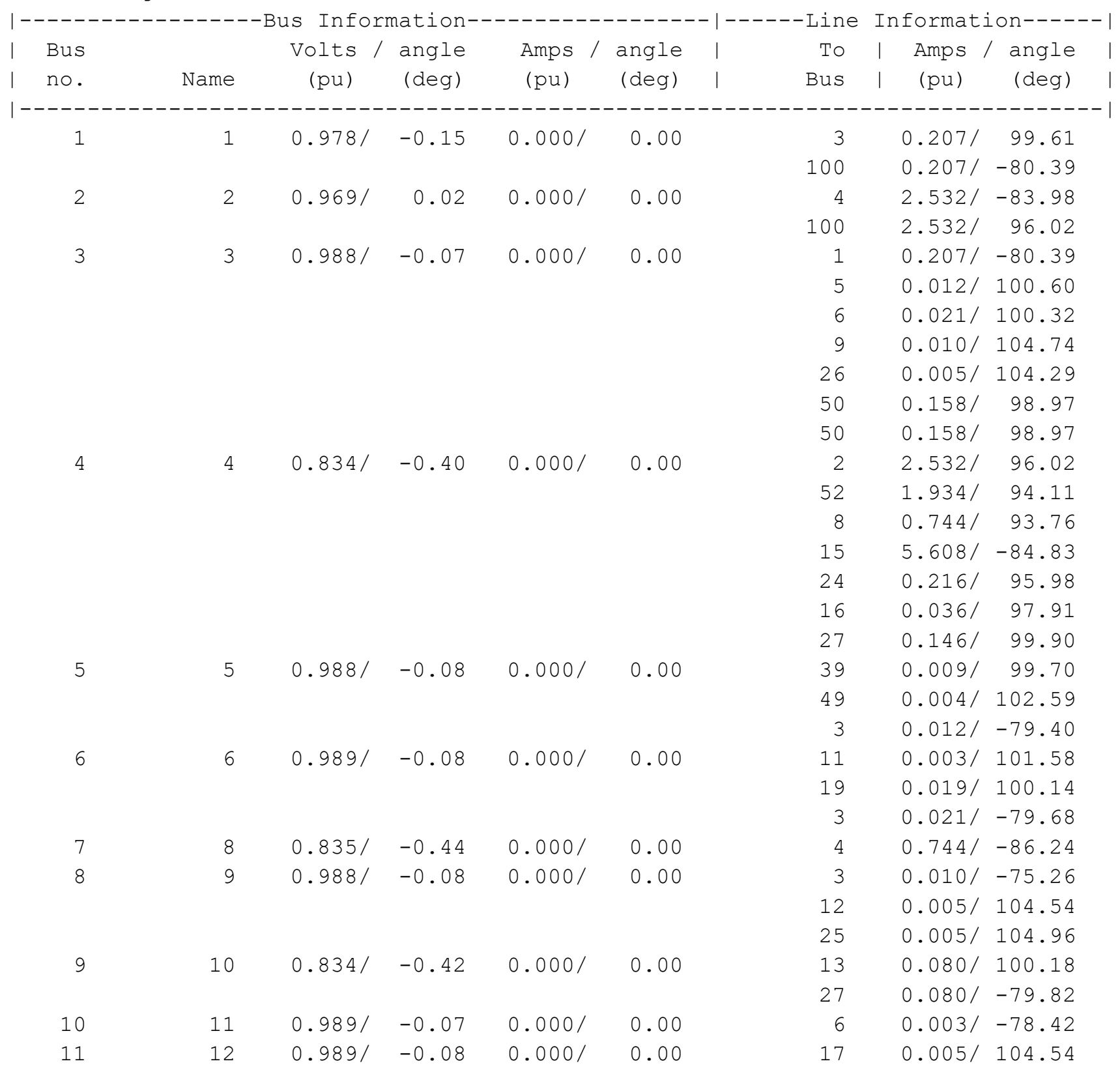




\begin{tabular}{|c|c|c|c|c|c|c|c|c|}
\hline 12 & 13 & $0.834 /$ & -0.42 & $0.000 /$ & 0.00 & 18 & $0.080 /$ & 100.18 \\
\hline & & & & & & 10 & $0.080 /$ & -79.82 \\
\hline 13 & 15 & $0.822 /$ & 0.41 & $0.000 /$ & 0.00 & 20 & $5.608 /$ & -84.83 \\
\hline & & & & & & 4 & $5.608 /$ & 95.17 \\
\hline 14 & 16 & $0.834 /$ & -0.41 & $0.000 /$ & 0.00 & 21 & $0.036 /$ & 97.91 \\
\hline & & & & & & 4 & $0.036 /$ & -82.09 \\
\hline 15 & 17 & $0.991 /$ & -0.06 & $0.000 /$ & 0.00 & 12 & $0.005 /$ & -75.46 \\
\hline & & & & & & 22 & $0.000 /$ & 100.83 \\
\hline 16 & 18 & $0.865 /$ & -0.36 & $0.000 /$ & 0.00 & 13 & $0.080 /$ & -79.82 \\
\hline & & & & & & 23 & $0.007 /$ & 96.47 \\
\hline 17 & 19 & $0.991 /$ & -0.06 & $0.000 /$ & 0.00 & 6 & $0.019 /$ & -79.86 \\
\hline 18 & 20 & $0.000 /$ & 0.00 & 7.2421 & -85.28 & 15 & $5.608 /$ & 95.17 \\
\hline 19 & 21 & $0.862 /$ & -0.50 & $0.000 /$ & 0.00 & 16 & $0.036 /$ & -82.09 \\
\hline 20 & 22 & $0.991 /$ & -0.06 & $0.000 /$ & 0.00 & 17 & $0.000 /$ & -79.17 \\
\hline 21 & 23 & $0.865 /$ & -0.37 & $0.000 /$ & 0.00 & 18 & $0.007 /$ & -83.53 \\
\hline 22 & 24 & $0.834 /$ & -0.42 & $0.000 /$ & 0.00 & 4 & $0.216 /$ & -84.02 \\
\hline & & & & & & 31 & $0.158 /$ & 94.76 \\
\hline & & & & & & 32 & $0.058 /$ & 99.25 \\
\hline 23 & 25 & $0.989 /$ & -0.08 & $0.000 /$ & 0.00 & 28 & $0.005 /$ & 104.96 \\
\hline & & & & & & 9 & $0.005 /$ & -75.04 \\
\hline 24 & 26 & $0.988 /$ & -0.08 & $0.000 /$ & 0.00 & 29 & $0.005 /$ & 104.29 \\
\hline & & & & & & 3 & $0.005 /$ & -75.71 \\
\hline 25 & 27 & $0.834 /$ & -0.41 & $0.000 /$ & 0.00 & 30 & $0.066 /$ & 99.57 \\
\hline & & & & & & 4 & $0.146 /$ & -80.10 \\
\hline & & & & & & 10 & $0.080 /$ & 100.18 \\
\hline 26 & 28 & $0.990 /$ & -0.06 & $0.000 /$ & 0.00 & 25 & $0.005 /$ & -75.04 \\
\hline & & & & & & 33 & $0.001 /$ & 101.58 \\
\hline & & & & & & 41 & $0.000 /$ & -121.54 \\
\hline 27 & 29 & $0.990 /$ & -0.06 & $0.000 /$ & 0.00 & 26 & $0.005 /$ & -75.71 \\
\hline & & & & & & 34 & $0.000 /$ & 104.80 \\
\hline & & & & & & 38 & $0.000 /$ & -133.94 \\
\hline & & & & & & 38 & $0.000 /$ & -133.94 \\
\hline 28 & 30 & $0.860 /$ & -0.38 & $0.000 /$ & 0.00 & 27 & $0.066 /$ & -80.43 \\
\hline & & & & & & 35 & $0.014 /$ & 97.29 \\
\hline 29 & 31 & $0.835 /$ & -0.43 & $0.000 /$ & 0.00 & 36 & $0.158 /$ & 94.76 \\
\hline & & & & & & 24 & $0.158 /$ & -85.24 \\
\hline 30 & 32 & $0.834 /$ & -0.42 & $0.000 /$ & 0.00 & 37 & $0.058 /$ & 99.25 \\
\hline & & & & & & 24 & $0.058 /$ & -80.75 \\
\hline 31 & 33 & $0.990 /$ & -0.07 & $0.000 /$ & 0.00 & 28 & $0.001 /$ & -78.42 \\
\hline 32 & 34 & $0.990 /$ & -0.07 & $0.000 /$ & 0.00 & 29 & $0.000 /$ & -75.20 \\
\hline 33 & 35 & $0.860 /$ & -0.41 & $0.000 /$ & 0.00 & 30 & $0.014 /$ & -82.71 \\
\hline 34 & 36 & $0.871 /$ & -0.45 & $0.000 /$ & 0.00 & 31 & $0.158 /$ & -85.24 \\
\hline 35 & 37 & $0.868 /$ & -0.45 & $0.000 /$ & 0.00 & 32 & $0.058 /$ & -80.75 \\
\hline 36 & 38 & $0.990 /$ & -0.06 & $0.000 /$ & 0.00 & 29 & $0.000 /$ & 46.06 \\
\hline & & & & & & 29 & $0.000 /$ & 46.06 \\
\hline 37 & 39 & $0.991 /$ & -0.07 & $0.000 /$ & 0.00 & 5 & $0.009 /$ & -80.30 \\
\hline 38 & 41 & $0.990 /$ & -0.06 & $0.000 /$ & 0.00 & 28 & $0.000 /$ & 58.46 \\
\hline 39 & 49 & $0.990 /$ & -0.07 & $0.000 /$ & 0.00 & 5 & $0.004 /$ & -77.41 \\
\hline 40 & 50 & $0.989 /$ & -0.08 & $0.000 /$ & 0.00 & 51 & $0.003 /$ & 103.54 \\
\hline
\end{tabular}




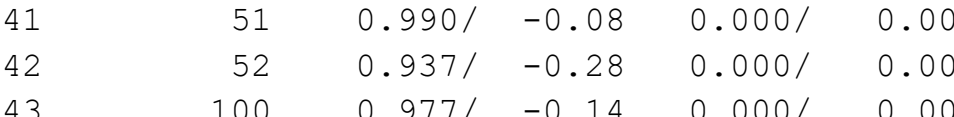

$30.158 /-81.03$

$30.158 /-81.03$

$50 \quad 0.003 /-76.46$

$4 \quad 1.934 /-85.89$

$10.207 / 99.61$

$2 \quad 2.532 /-83.98$

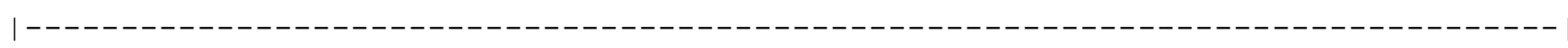




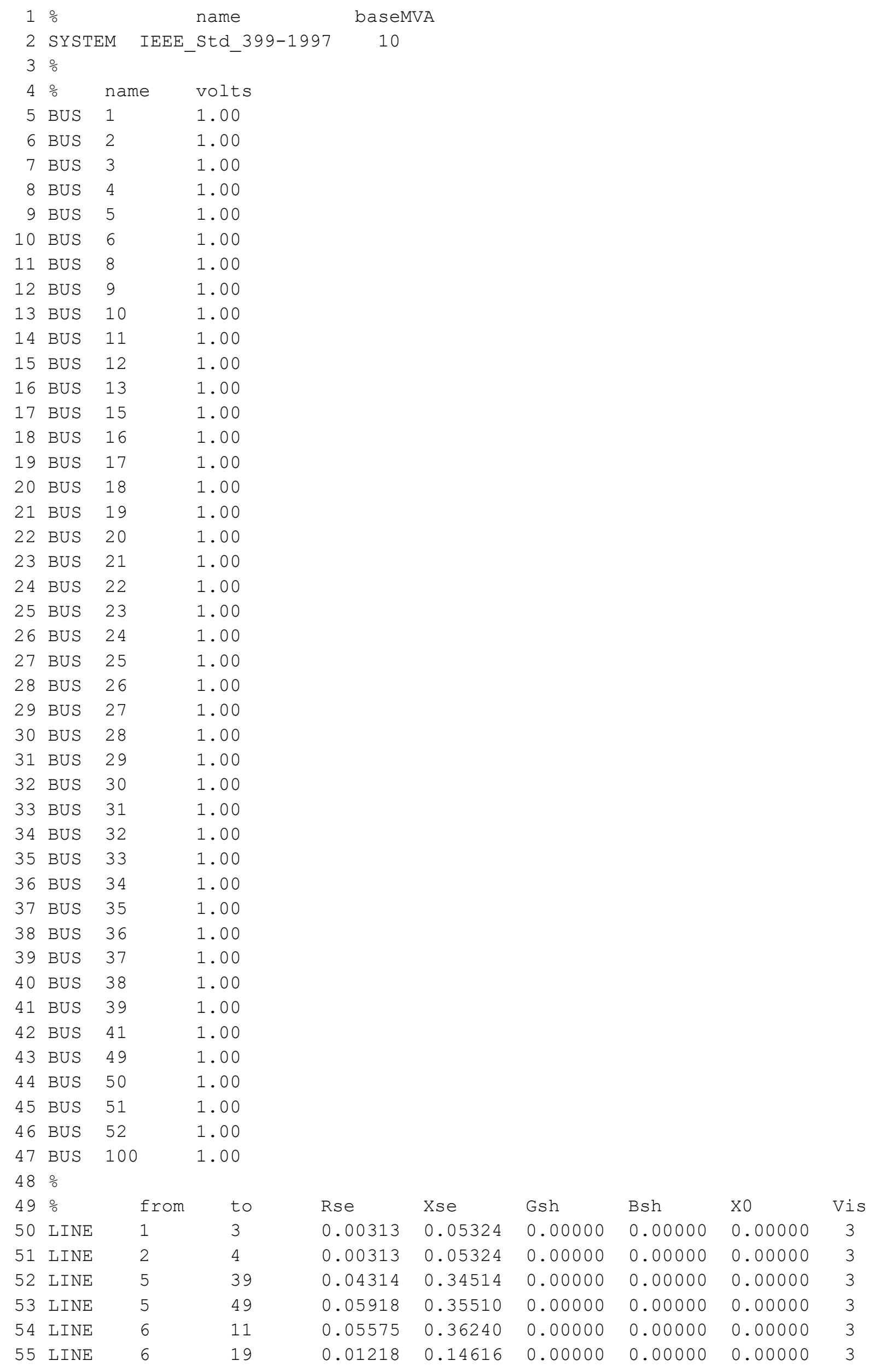




\begin{tabular}{|c|c|c|c|c|c|c|c|c|}
\hline 56 & LINE & 12 & 17 & 0.06843 & 0.44477 & 0.00000 & 0.00000 & 0.00000 \\
\hline 57 & LINE & 13 & 18 & 0.05829 & 0.37888 & 0.00000 & 0.00000 & 0.00000 \\
\hline 58 & LINE & 15 & 20 & 0.01218 & 0.14616 & 0.00000 & 0.00000 & 0.00000 \\
\hline 59 & LINE & 16 & 21 & 0.15036 & 0.75178 & 0.00000 & 0.00000 & 0.00000 \\
\hline 60 & LINE & 25 & 28 & 0.05829 & 0.37888 & 0.00000 & 0.00000 & 0.00000 \\
\hline 61 & LINE & 26 & 29 & 0.05829 & 0.37888 & 0.00000 & 0.00000 & 0.00000 \\
\hline 62 & LINE & 27 & 30 & 0.05829 & 0.37888 & 0.00000 & 0.00000 & 0.00000 \\
\hline 63 & LINE & 31 & 36 & 0.02289 & 0.22886 & 0.00000 & 0.00000 & 0.00000 \\
\hline 64 & LINE & 32 & 37 & 0.10286 & 0.56573 & 0.00000 & 0.00000 & 0.00000 \\
\hline 65 & LINE & 50 & 51 & 0.06395 & 0.37796 & 0.00000 & 0.00000 & 0.00000 \\
\hline 66 & LINE & 52 & 4 & 0.00313 & 0.05324 & 0.00000 & 0.00000 & 0.00000 \\
\hline 67 & LINE & 3 & 5 & 0.00075 & 0.00063 & 0.00000 & 0.00000 & 0.00000 \\
\hline 68 & LINE & 3 & 6 & 0.00109 & 0.00091 & 0.00000 & 0.00000 & 0.00000 \\
\hline 69 & LINE & 3 & 9 & 0.00150 & 0.00125 & 0.00000 & 0.00000 & 0.00000 \\
\hline 70 & LINE & 3 & 26 & 0.00157 & 0.00131 & 0.00000 & 0.00000 & 0.00000 \\
\hline 71 & LINE & 4 & 8 & 0.00076 & 0.00092 & 0.00000 & 0.00000 & 0.00000 \\
\hline 72 & LINE & 4 & 15 & 0.00227 & 0.00189 & 0.00000 & 0.00000 & 0.00000 \\
\hline 73 & LINE & 4 & 24 & 0.00118 & 0.00098 & 0.00000 & 0.00000 & 0.00000 \\
\hline 74 & LINE & 4 & 16 & 0.00274 & 0.00229 & 0.00000 & 0.00000 & 0.00000 \\
\hline 75 & LINE & 4 & 27 & 0.00143 & 0.00119 & 0.00000 & 0.00000 & 0.00000 \\
\hline 76 & LINE & 9 & 12 & 0.00038 & 0.00032 & 0.00000 & 0.00000 & 0.00000 \\
\hline 77 & LINE & 9 & 25 & 0.00424 & 0.00353 & 0.00000 & 0.00000 & 0.00000 \\
\hline 78 & LINE & 10 & 13 & 0.00046 & 0.00039 & 0.00000 & 0.00000 & 0.00000 \\
\hline 79 & LINE & 10 & 27 & 0.00110 & 0.00091 & 0.00000 & 0.00000 & 0.00000 \\
\hline 80 & LINE & 17 & 22 & 0.03813 & 0.02451 & 0.00000 & 0.00000 & 0.00000 \\
\hline 81 & LINE & 18 & 23 & 0.03813 & 0.02451 & 0.00000 & 0.00000 & 0.00000 \\
\hline 82 & LINE & 24 & 31 & 0.00079 & 0.00065 & 0.00000 & 0.00000 & 0.00000 \\
\hline 83 & LINE & 24 & 32 & 0.00112 & 0.00093 & 0.00000 & 0.00000 & 0.00000 \\
\hline 84 & LINE & 28 & 33 & 0.03813 & 0.02451 & 0.00000 & 0.00000 & 0.00000 \\
\hline 85 & LINE & 28 & 41 & 0.03429 & 0.02105 & 0.00000 & 0.00000 & 0.00000 \\
\hline 86 & LINE & 29 & 34 & 0.03813 & 0.02451 & 0.00000 & 0.00000 & 0.00000 \\
\hline 87 & LINE & 29 & 38 & 0.08024 & 0.07732 & 0.00000 & 0.00000 & 0.00000 \\
\hline 88 & LINE & 29 & 38 & 0.08024 & 0.07732 & 0.00000 & 0.00000 & 0.00000 \\
\hline 89 & LINE & 30 & 35 & 0.03813 & 0.02451 & 0.00000 & 0.00000 & 0.00000 \\
\hline 90 & LINE & 50 & 3 & 0.00243 & 0.00485 & 0.00000 & 0.00000 & 0.00000 \\
\hline 91 & LINE & 50 & 3 & 0.00243 & 0.00485 & 0.00000 & 0.00000 & 0.00000 \\
\hline 92 & LINE & 100 & 1 & 0.00139 & 0.00296 & 0.00000 & 0.00000 & 0.00000 \\
\hline 93 & LINE & 100 & 2 & 0.00139 & 0.00296 & 0.00000 & 0.00000 & 0.00000 \\
\hline
\end{tabular}

\begin{tabular}{|c|c|c|c|c|c|c|c|}
\hline $95 \div$ & bus & $\mathrm{R}$ & Xs & $\mathrm{Xp}$ & Xpp & $\mathrm{x} 2$ & xo \\
\hline 96 GENERATOR & 50 & 0.002 & 0.000 & 0.000 & 0.072 & 0.000 & 0.000 \\
\hline 97 GENERATOR & 52 & 0.000 & 0.000 & 0.000 & 0.0131 & 0.000 & 0.000 \\
\hline 98 GENERATOR & 100 & 0.000 & 0.000 & 0.000 & 0.010 & 0.000 & 0.000 \\
\hline $99 \div$ & & & & & & & \\
\hline $100 \%$ & bus & $\mathrm{R}$ & $X s$ & $\mathrm{xp}$ & Xpp & $\mathrm{x} 2$ & xo \\
\hline 101 MOTOR & 11 & 0.352 & 0.000 & 0.000 & 4.219 & 0.000 & 0.000 \\
\hline 102 MOTOR & 17 & 0.338 & 0.000 & 0.000 & 3.384 & 0.000 & 0.000 \\
\hline 103 MOTOR & 17 & 0.802 & 0.000 & 0.000 & 4.008 & 0.000 & 0.000 \\
\hline 104 MOTOR & 18 & 0.338 & 0.000 & 0.000 & 3.384 & 0.000 & 0.000 \\
\hline 105 MOTOR & 18 & 0.802 & 0.000 & 0.000 & 4.008 & 0.000 & 0.000 \\
\hline 106 MOTOR & 19 & 0.057 & 0.000 & 0.000 & 1.484 & 0.000 & 0.000 \\
\hline 107 MOTOR & 19 & 0.047 & 0.000 & 0.000 & 0.703 & 0.000 & 0.000 \\
\hline 108 MOTOR & 20 & 0.067 & 0.000 & 0.000 & 1.005 & 0.000 & 0.000 \\
\hline 109 MOTOR & 20 & 0.060 & 0.000 & 0.000 & 1.556 & 0.000 & 0.000 \\
\hline MOTOR & 21 & 0.320 & 0.000 & 0.000 & 3.835 & 0.000 & 0.000 \\
\hline
\end{tabular}




\begin{tabular}{|c|c|c|c|c|c|c|c|c|c|}
\hline 111 & MOTOR & & 22 & 1.398 & 0.000 & 0.000 & 19.571 & 0.000 & 0.000 \\
\hline 112 & MOTOR & & 23 & 1.398 & 0.000 & 0.000 & 19.571 & 0.000 & 0.000 \\
\hline 113 & MOTOR & & 28 & 0.697 & 0.000 & 0.000 & 6.972 & 0.000 & 0.000 \\
\hline 114 & MOTOR & & 28 & 0.802 & 0.000 & 0.000 & 4.008 & 0.000 & 0.000 \\
\hline 115 & MOTOR & & 29 & 0.321 & 0.000 & 0.000 & 3.206 & 0.000 & 0.000 \\
\hline 116 & MOTOR & & 29 & 1.199 & 0.000 & 0.000 & 5.998 & 0.000 & 0.000 \\
\hline 117 & MOTOR & & 30 & 0.431 & 0.000 & 0.000 & 5.166 & 0.000 & 0.000 \\
\hline 118 & MOTOR & & 30 & 1.116 & 0.000 & 0.000 & 5.578 & 0.000 & 0.000 \\
\hline 119 & MOTOR & & 33 & 0.809 & 0.000 & 0.000 & 9.701 & 0.000 & 0.000 \\
\hline 120 & MOTOR & & 34 & 3.621 & 0.000 & 0.000 & 25.354 & 0.000 & 0.000 \\
\hline 121 & MOTOR & & 35 & 0.809 & 0.000 & 0.000 & 9.701 & 0.000 & 0.000 \\
\hline 122 & MOTOR & & 36 & 0.025 & 0.000 & 0.000 & 0.818 & 0.000 & 0.000 \\
\hline 123 & MOTOR & & 37 & 0.246 & 0.000 & 0.000 & 2.952 & 0.000 & 0.000 \\
\hline 124 & MOTOR & & 37 & 1.859 & 0.000 & 0.000 & 9.296 & 0.000 & 0.000 \\
\hline 125 & MOTOR & & 39 & 0.034 & 0.000 & 0.000 & 1.005 & 0.000 & 0.000 \\
\hline 126 & MOTOR & & 49 & 0.264 & 0.000 & 0.000 & 2.640 & 0.000 & 0.000 \\
\hline 127 & MOTOR & & 51 & 1.432 & 0.000 & 0.000 & 10.020 & 0.000 & 0.000 \\
\hline 128 & MOTOR & & 51 & 0.408 & 0.000 & 0.000 & 4.893 & 0.000 & 0.000 \\
\hline 129 & MOTOR & & 8 & 0.006 & 0.000 & 0.000 & 0.222 & 0.000 & 0.000 \\
\hline 130 & $\%$ & & & & & & & & \\
\hline 131 & \% & bus & Calc & Type & Lc_time & $(\Omega-A]$ & $1-\mathrm{S}$ ibt & nsien & ;2-Transient;3-Steady-state \\
\hline 132 & FAULT & 20 & 3 & & $\overline{1}$ & & & & \\
\hline
\end{tabular}




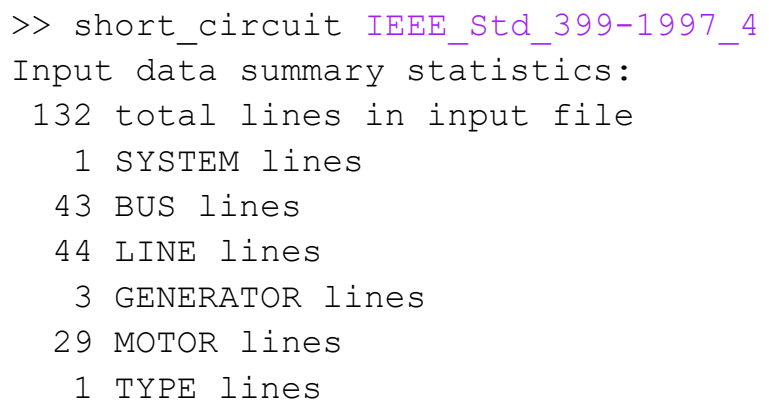

Results for System Name "IEEE_Std_399-1997"

Symmetrical Three-Phase Fault at Bus 20

Calculating Subtransient Currents

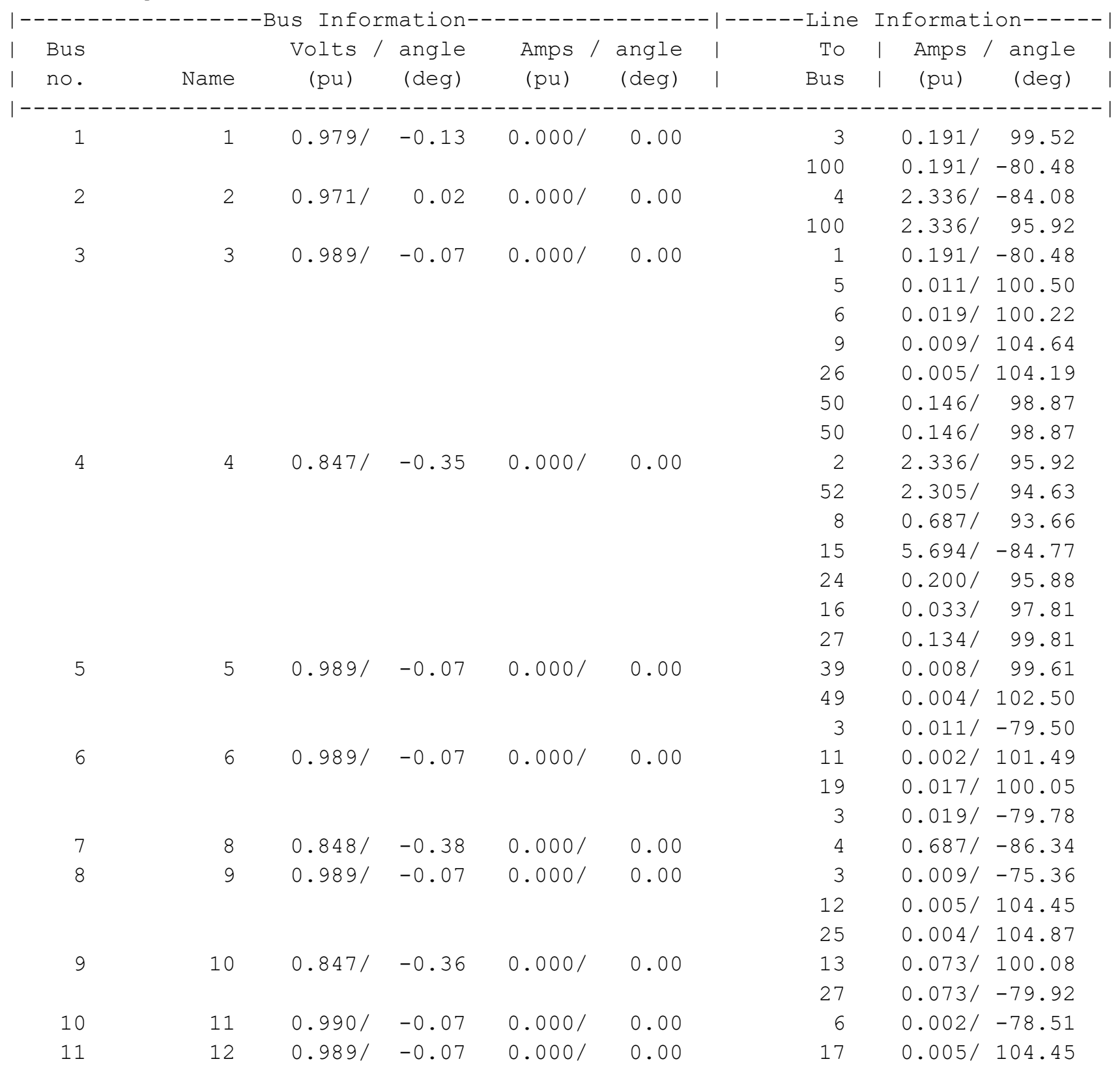




\begin{tabular}{|c|c|c|c|c|c|c|c|c|}
\hline \multirow[t]{2}{*}{12} & 13 & $0.847 /$ & -0.37 & $0.000 /$ & 0.00 & 18 & $0.073 /$ & 100.08 \\
\hline & & & & & & 10 & $0.073 /$ & -79.92 \\
\hline \multirow[t]{2}{*}{13} & 15 & $0.835 /$ & 0.46 & $0.000 /$ & 0.00 & 20 & $5.694 /$ & -84.77 \\
\hline & & & & & & 4 & $5.694 /$ & 95.23 \\
\hline \multirow[t]{2}{*}{14} & 16 & $0.847 /$ & -0.35 & $0.000 /$ & 0.00 & 21 & $0.033 /$ & 97.81 \\
\hline & & & & & & 4 & $0.033 /$ & -82.19 \\
\hline \multirow[t]{2}{*}{15} & 17 & $0.992 /$ & -0.06 & $0.000 /$ & 0.00 & 12 & $0.005 /$ & -75.55 \\
\hline & & & & & & 22 & $0.000 /$ & 100.74 \\
\hline \multirow[t]{2}{*}{16} & 18 & $0.875 /$ & -0.31 & $0.000 /$ & 0.00 & 13 & $0.073 /$ & -79.92 \\
\hline & & & & & & 23 & $0.006 /$ & 96.37 \\
\hline 17 & 19 & $0.992 /$ & -0.06 & $0.000 /$ & 0.00 & 6 & $0.017 /$ & -79.95 \\
\hline 18 & 20 & $0.000 /$ & 0.00 & $7.328 /$ & -85.23 & 15 & $5.694 /$ & 95.23 \\
\hline 19 & 21 & $0.872 /$ & -0.44 & $0.000 /$ & 0.00 & 16 & $0.033 /$ & -82.19 \\
\hline 20 & 22 & $0.992 /$ & -0.06 & $0.000 /$ & 0.00 & 17 & $0.000 /$ & -79.26 \\
\hline 21 & 23 & $0.876 /$ & -0.33 & $0.000 /$ & 0.00 & 18 & $0.006 /$ & -83.63 \\
\hline \multirow[t]{3}{*}{22} & 24 & $0.847 /$ & -0.36 & $0.000 /$ & 0.00 & 4 & $0.200 /$ & -84.12 \\
\hline & & & & & & 31 & $0.146 /$ & 94.67 \\
\hline & & & & & & 32 & $0.054 /$ & 99.16 \\
\hline \multirow[t]{2}{*}{23} & 25 & $0.989 /$ & -0.07 & $0.000 /$ & 0.00 & 28 & $0.004 /$ & 104.87 \\
\hline & & & & & & 9 & $0.004 /$ & -75.13 \\
\hline \multirow[t]{2}{*}{24} & 26 & $0.989 /$ & -0.07 & $0.000 /$ & 0.00 & 29 & $0.005 /$ & 104.19 \\
\hline & & & & & & 3 & $0.005 /$ & -75.81 \\
\hline \multirow[t]{3}{*}{25} & 27 & $0.847 /$ & -0.36 & $0.000 /$ & 0.00 & 30 & $0.061 /$ & 99.47 \\
\hline & & & & & & 4 & $0.134 /$ & -80.19 \\
\hline & & & & & & 10 & $0.073 /$ & 100.08 \\
\hline \multirow[t]{3}{*}{26} & 28 & $0.991 /$ & -0.06 & $0.000 /$ & 0.00 & 25 & $0.004 /$ & -75.13 \\
\hline & & & & & & 33 & $0.001 /$ & 101.49 \\
\hline & & & & & & 41 & $0.000 /-$ & -121.54 \\
\hline \multirow[t]{4}{*}{27} & 29 & $0.991 /$ & -0.06 & $0.000 /$ & 0.00 & 26 & $0.005 /$ & -75.81 \\
\hline & & & & & & 34 & $0.000 /$ & 104.71 \\
\hline & & & & & & 38 & $0.000 /-$ & -133.94 \\
\hline & & & & & & 38 & $0.000 /-$ & -133.94 \\
\hline \multirow[t]{2}{*}{28} & 30 & $0.871 /$ & -0.33 & $0.000 /$ & 0.00 & 27 & $0.061 /$ & -80.53 \\
\hline & & & & & & 35 & $0.013 /$ & 97.19 \\
\hline \multirow[t]{2}{*}{29} & 31 & $0.847 /$ & -0.37 & $0.000 /$ & 0.00 & 36 & $0.146 /$ & 94.67 \\
\hline & & & & & & 24 & $0.146 /$ & -85.33 \\
\hline \multirow[t]{2}{*}{30} & 32 & $0.847 /$ & -0.37 & $0.000 /$ & 0.00 & 37 & $0.054 /$ & 99.16 \\
\hline & & & & & & 24 & $0.054 /$ & -80.84 \\
\hline 31 & 33 & $0.991 /$ & -0.06 & $0.000 /$ & 0.00 & 28 & $0.001 /$ & -78.51 \\
\hline 32 & 34 & $0.991 /$ & -0.06 & $0.000 /$ & 0.00 & 29 & $0.000 /$ & -75.29 \\
\hline 33 & 35 & $0.871 /$ & -0.36 & $0.000 /$ & 0.00 & 30 & $0.013 /$ & -82.81 \\
\hline 34 & 36 & $0.881 /$ & -0.40 & $0.000 /$ & 0.00 & 31 & $0.146 /$ & -85.33 \\
\hline 35 & 37 & $0.878 /$ & -0.39 & $0.000 /$ & 0.00 & 32 & $0.054 /$ & -80.84 \\
\hline \multirow[t]{2}{*}{36} & 38 & $0.991 /$ & -0.06 & $0.000 /$ & 0.00 & 29 & $0.000 /$ & 46.06 \\
\hline & & & & & & 29 & $0.000 /$ & 46.06 \\
\hline 37 & 39 & $0.992 /$ & -0.06 & $0.000 /$ & 0.00 & 5 & $0.008 /$ & -80.39 \\
\hline 38 & 41 & $0.991 /$ & -0.06 & $0.000 /$ & 0.00 & 28 & $0.000 /$ & 58.46 \\
\hline 39 & 49 & $0.991 /$ & -0.06 & $0.000 /$ & 0.00 & 5 & $0.004 /$ & -77.50 \\
\hline 40 & 50 & $0.990 /$ & -0.07 & $0.000 /$ & 0.00 & 51 & $0.003 /$ & 103.45 \\
\hline
\end{tabular}




$\begin{array}{rrrrrr}42 & 52 & 0.970 / & -0.14 & 0.000 / & 0.00 \\ 43 & 100 & 0.979 / & -0.12 & 0.000 / & 0.00\end{array}$

$30.146 /-81.13$

$30.146 /-81.13$

41

43

$0.000 /$

50

$0.003 /-76.55$

$4 \quad 2.305 /-85.37$

$10.191 / 99.52$

$2 \quad 2.336 /-84.08$

$>>$ 


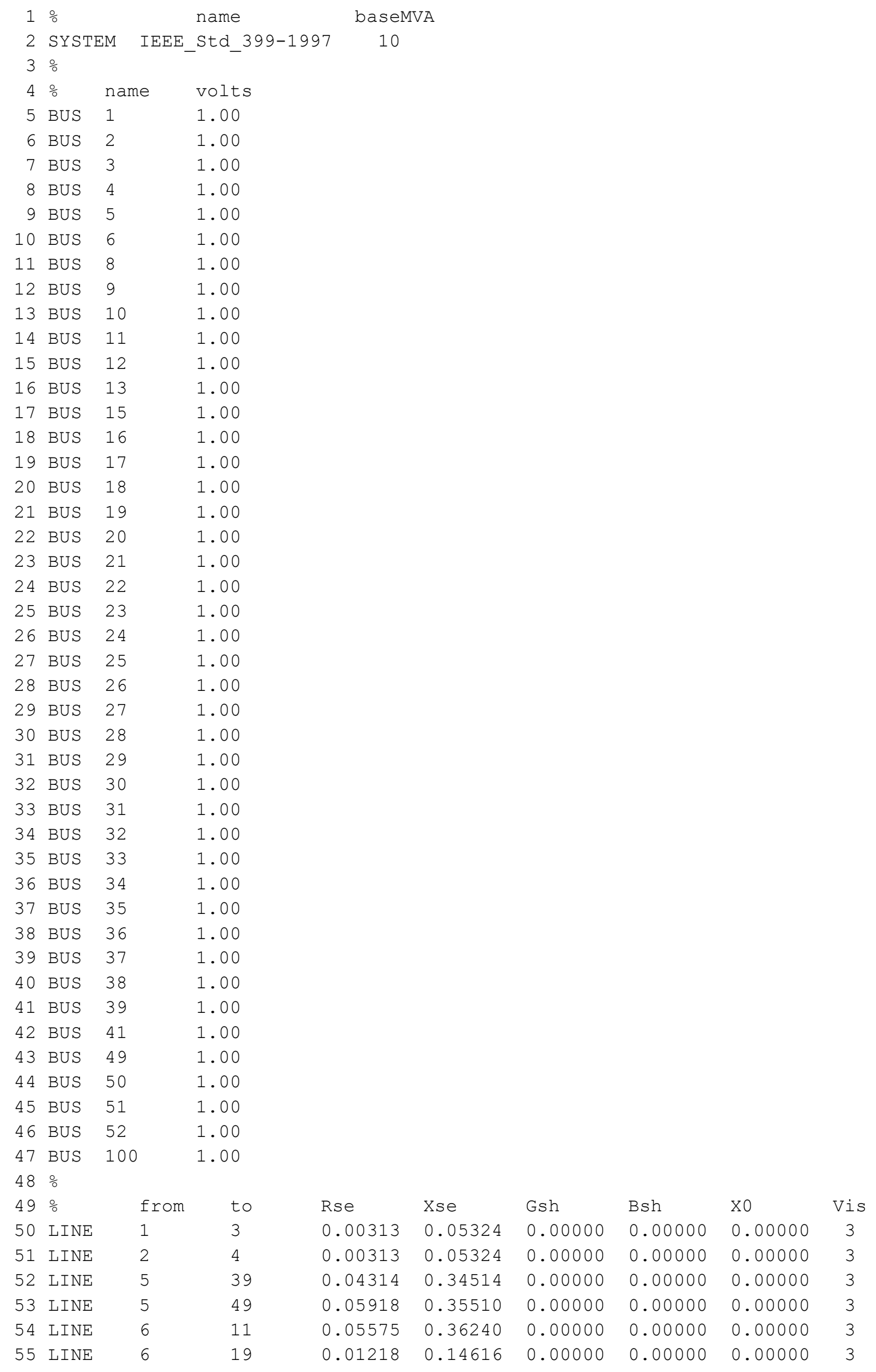




\begin{tabular}{|c|c|c|c|c|c|c|c|c|}
\hline 56 & LINE & 12 & 17 & 0.06843 & 0.44477 & 0.00000 & 0.00000 & 0.00000 \\
\hline 57 & LINE & 13 & 18 & 0.05829 & 0.37888 & 0.00000 & 0.00000 & 0.00000 \\
\hline 58 & LINE & 15 & 20 & 0.01218 & 0.14616 & 0.00000 & 0.00000 & 0.00000 \\
\hline 59 & LINE & 16 & 21 & 0.15036 & 0.75178 & 0.00000 & 0.00000 & 0.00000 \\
\hline 60 & LINE & 25 & 28 & 0.05829 & 0.37888 & 0.00000 & 0.00000 & 0.00000 \\
\hline 61 & LINE & 26 & 29 & 0.05829 & 0.37888 & 0.00000 & 0.00000 & 0.00000 \\
\hline 62 & LINE & 27 & 30 & 0.05829 & 0.37888 & 0.00000 & 0.00000 & 0.00000 \\
\hline 63 & LINE & 31 & 36 & 0.02289 & 0.22886 & 0.00000 & 0.00000 & 0.00000 \\
\hline 64 & LINE & 32 & 37 & 0.10286 & 0.56573 & 0.00000 & 0.00000 & 0.00000 \\
\hline 65 & LINE & 50 & 51 & 0.06395 & 0.37796 & 0.00000 & 0.00000 & 0.00000 \\
\hline 66 & LINE & 52 & 4 & 0.00313 & 0.05324 & 0.00000 & 0.00000 & 0.00000 \\
\hline 67 & LINE & 3 & 5 & 0.00075 & 0.00063 & 0.00000 & 0.00000 & 0.00000 \\
\hline 68 & LINE & 3 & 6 & 0.00109 & 0.00091 & 0.00000 & 0.00000 & 0.00000 \\
\hline 69 & LINE & 3 & 9 & 0.00150 & 0.00125 & 0.00000 & 0.00000 & 0.00000 \\
\hline 70 & LINE & 3 & 26 & 0.00157 & 0.00131 & 0.00000 & 0.00000 & 0.00000 \\
\hline 71 & LINE & 4 & 8 & 0.00076 & 0.00092 & 0.00000 & 0.00000 & 0.00000 \\
\hline 72 & LINE & 4 & 15 & 0.00227 & 0.00189 & 0.00000 & 0.00000 & 0.00000 \\
\hline 73 & LINE & 4 & 24 & 0.00118 & 0.00098 & 0.00000 & 0.00000 & 0.00000 \\
\hline 74 & LINE & 4 & 16 & 0.00274 & 0.00229 & 0.00000 & 0.00000 & 0.00000 \\
\hline 75 & LINE & 4 & 27 & 0.00143 & 0.00119 & 0.00000 & 0.00000 & 0.00000 \\
\hline 76 & LINE & 9 & 12 & 0.00038 & 0.00032 & 0.00000 & 0.00000 & 0.00000 \\
\hline 77 & LINE & 9 & 25 & 0.00424 & 0.00353 & 0.00000 & 0.00000 & 0.00000 \\
\hline 78 & LINE & 10 & 13 & 0.00046 & 0.00039 & 0.00000 & 0.00000 & 0.00000 \\
\hline 79 & LINE & 10 & 27 & 0.00110 & 0.00091 & 0.00000 & 0.00000 & 0.00000 \\
\hline 80 & LINE & 17 & 22 & 0.03813 & 0.02451 & 0.00000 & 0.00000 & 0.00000 \\
\hline 81 & LINE & 18 & 23 & 0.03813 & 0.02451 & 0.00000 & 0.00000 & 0.00000 \\
\hline 82 & LINE & 24 & 31 & 0.00079 & 0.00065 & 0.00000 & 0.00000 & 0.00000 \\
\hline 83 & LINE & 24 & 32 & 0.00112 & 0.00093 & 0.00000 & 0.00000 & 0.00000 \\
\hline 84 & LINE & 28 & 33 & 0.03813 & 0.02451 & 0.00000 & 0.00000 & 0.00000 \\
\hline 85 & LINE & 28 & 41 & 0.03429 & 0.02105 & 0.00000 & 0.00000 & 0.00000 \\
\hline 86 & LINE & 29 & 34 & 0.03813 & 0.02451 & 0.00000 & 0.00000 & 0.00000 \\
\hline 87 & LINE & 29 & 38 & 0.08024 & 0.07732 & 0.00000 & 0.00000 & 0.00000 \\
\hline 88 & LINE & 29 & 38 & 0.08024 & 0.07732 & 0.00000 & 0.00000 & 0.00000 \\
\hline 89 & LINE & 30 & 35 & 0.03813 & 0.02451 & 0.00000 & 0.00000 & 0.00000 \\
\hline 90 & LINE & 50 & 3 & 0.00243 & 0.00485 & 0.00000 & 0.00000 & 0.00000 \\
\hline 91 & LINE & 50 & 3 & 0.00243 & 0.00485 & 0.00000 & 0.00000 & 0.00000 \\
\hline 92 & LINE & 100 & 1 & 0.00139 & 0.00296 & 0.00000 & 0.00000 & 0.00000 \\
\hline 93 & LINE & 100 & 2 & 0.00139 & 0.00296 & 0.00000 & 0.00000 & 0.00000 \\
\hline
\end{tabular}

\begin{tabular}{|c|c|c|c|c|c|c|c|}
\hline $95 \div$ & bus & $\mathrm{R}$ & Xs & $\mathrm{Xp}$ & Xpp & $\mathrm{x} 2$ & xo \\
\hline 96 GENERATOR & 50 & 0.002 & 0.000 & 0.000 & 0.072 & 0.000 & 0.000 \\
\hline 97 GENERATOR & 52 & 0.000 & 0.000 & 0.000 & 0.0079 & 0.000 & 0.000 \\
\hline 98 GENERATOR & 100 & 0.000 & 0.000 & 0.000 & 0.010 & 0.000 & 0.000 \\
\hline $99 \div$ & & & & & & & \\
\hline $100 \%$ & bus & $\mathrm{R}$ & $X s$ & $\mathrm{xp}$ & Xpp & $\mathrm{x} 2$ & xo \\
\hline 101 MOTOR & 11 & 0.352 & 0.000 & 0.000 & 4.219 & 0.000 & 0.000 \\
\hline 102 MOTOR & 17 & 0.338 & 0.000 & 0.000 & 3.384 & 0.000 & 0.000 \\
\hline 103 MOTOR & 17 & 0.802 & 0.000 & 0.000 & 4.008 & 0.000 & 0.000 \\
\hline 104 MOTOR & 18 & 0.338 & 0.000 & 0.000 & 3.384 & 0.000 & 0.000 \\
\hline 105 MOTOR & 18 & 0.802 & 0.000 & 0.000 & 4.008 & 0.000 & 0.000 \\
\hline 106 MOTOR & 19 & 0.057 & 0.000 & 0.000 & 1.484 & 0.000 & 0.000 \\
\hline 107 MOTOR & 19 & 0.047 & 0.000 & 0.000 & 0.703 & 0.000 & 0.000 \\
\hline 108 MOTOR & 20 & 0.067 & 0.000 & 0.000 & 1.005 & 0.000 & 0.000 \\
\hline 109 MOTOR & 20 & 0.060 & 0.000 & 0.000 & 1.556 & 0.000 & 0.000 \\
\hline MOTOR & 21 & 0.320 & 0.000 & 0.000 & 3.835 & 0.000 & 0.000 \\
\hline
\end{tabular}




\begin{tabular}{|c|c|c|c|c|c|c|c|c|c|}
\hline 111 & MOTOR & & 22 & 1.398 & 0.000 & 0.000 & 19.571 & 0.000 & 0.000 \\
\hline 112 & MOTOR & & 23 & 1.398 & 0.000 & 0.000 & 19.571 & 0.000 & 0.000 \\
\hline 113 & MOTOR & & 28 & 0.697 & 0.000 & 0.000 & 6.972 & 0.000 & 0.000 \\
\hline 114 & MOTOR & & 28 & 0.802 & 0.000 & 0.000 & 4.008 & 0.000 & 0.000 \\
\hline 115 & MOTOR & & 29 & 0.321 & 0.000 & 0.000 & 3.206 & 0.000 & 0.000 \\
\hline 116 & MOTOR & & 29 & 1.199 & 0.000 & 0.000 & 5.998 & 0.000 & 0.000 \\
\hline 117 & MOTOR & & 30 & 0.431 & 0.000 & 0.000 & 5.166 & 0.000 & 0.000 \\
\hline 118 & MOTOR & & 30 & 1.116 & 0.000 & 0.000 & 5.578 & 0.000 & 0.000 \\
\hline 119 & MOTOR & & 33 & 0.809 & 0.000 & 0.000 & 9.701 & 0.000 & 0.000 \\
\hline 120 & MOTOR & & 34 & 3.621 & 0.000 & 0.000 & 25.354 & 0.000 & 0.000 \\
\hline 121 & MOTOR & & 35 & 0.809 & 0.000 & 0.000 & 9.701 & 0.000 & 0.000 \\
\hline 122 & MOTOR & & 36 & 0.025 & 0.000 & 0.000 & 0.818 & 0.000 & 0.000 \\
\hline 123 & MOTOR & & 37 & 0.246 & 0.000 & 0.000 & 2.952 & 0.000 & 0.000 \\
\hline 124 & MOTOR & & 37 & 1.859 & 0.000 & 0.000 & 9.296 & 0.000 & 0.000 \\
\hline 125 & MOTOR & & 39 & 0.034 & 0.000 & 0.000 & 1.005 & 0.000 & 0.000 \\
\hline 126 & MOTOR & & 49 & 0.264 & 0.000 & 0.000 & 2.640 & 0.000 & 0.000 \\
\hline 127 & MOTOR & & 51 & 1.432 & 0.000 & 0.000 & 10.020 & 0.000 & 0.000 \\
\hline 128 & MOTOR & & 51 & 0.408 & 0.000 & 0.000 & 4.893 & 0.000 & 0.000 \\
\hline 129 & MOTOR & & 8 & 0.006 & 0.000 & 0.000 & 0.222 & 0.000 & 0.000 \\
\hline 130 & $\%$ & & & & & & & & \\
\hline 131 & \% & bus & Calc & Type & Lc_time & $(\Omega-A]$ & $1-\mathrm{S}$ ibt & nsien & ;2-Transient;3-Steady-state \\
\hline 132 & FAULT & 20 & 3 & & $\overline{1}$ & & & & \\
\hline
\end{tabular}




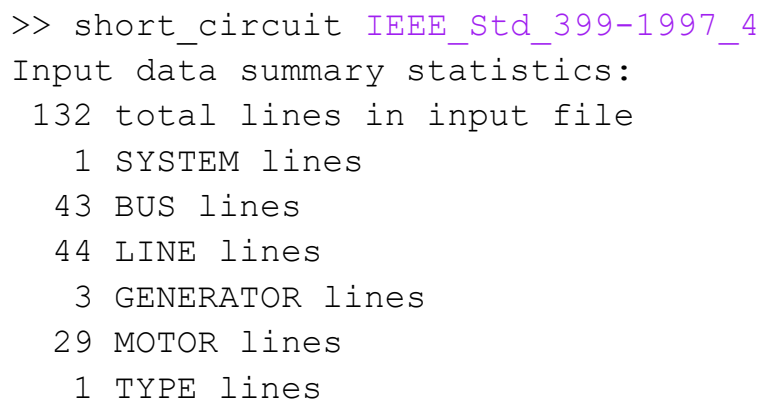

Results for System Name "IEEE_Std_399-1997"

Symmetrical Three-Phase Fault at Bus 20

Calculating Subtransient Currents

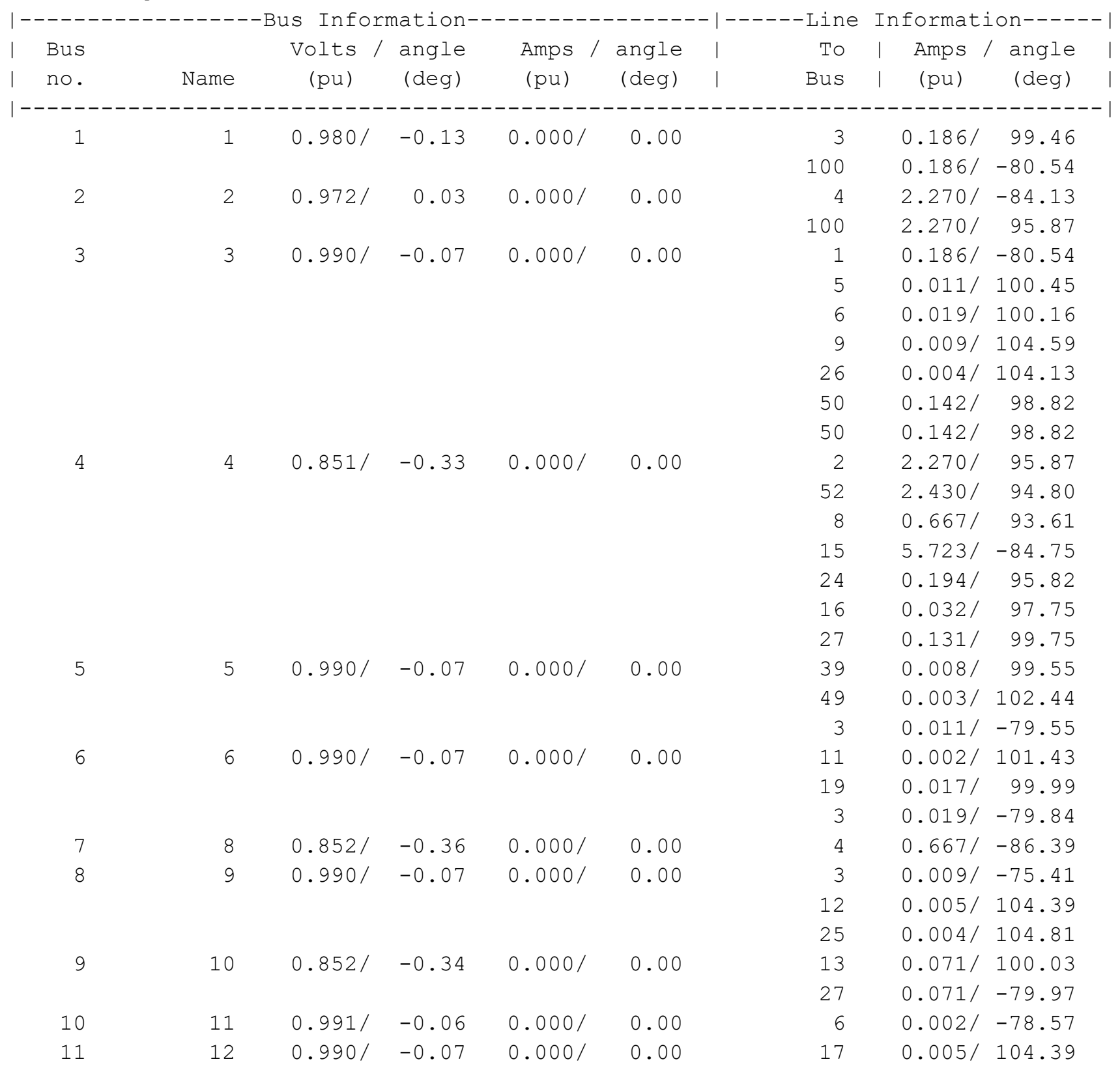




\begin{tabular}{|c|c|c|c|c|c|c|c|c|}
\hline & & & & & & 9 & $0.005 /$ & -75.61 \\
\hline \multirow[t]{2}{*}{12} & 13 & $0.852 /$ & -0.34 & $0.000 /$ & 0.00 & 18 & $0.071 /$ & 100.03 \\
\hline & & & & & & 10 & $0.071 /$ & -79.97 \\
\hline \multirow[t]{2}{*}{13} & 15 & $0.839 /$ & 0.48 & $0.000 /$ & 0.00 & 20 & $5.723 /$ & -84.75 \\
\hline & & & & & & 4 & $5.723 /$ & 95.25 \\
\hline \multirow[t]{2}{*}{14} & 16 & $0.851 /$ & -0.33 & $0.000 /$ & 0.00 & 21 & $0.032 /$ & 97.75 \\
\hline & & & & & & 4 & $0.032 /$ & -82.25 \\
\hline \multirow[t]{2}{*}{15} & 17 & $0.992 /$ & -0.05 & $0.000 /$ & 0.00 & 12 & $0.005 /$ & -75.61 \\
\hline & & & & & & 22 & $0.000 /$ & 100.68 \\
\hline \multirow[t]{2}{*}{16} & 18 & $0.879 /$ & -0.29 & $0.000 /$ & 0.00 & 13 & $0.071 /$ & -79.97 \\
\hline & & & & & & 23 & $0.006 /$ & 96.32 \\
\hline 17 & 19 & $0.992 /$ & -0.05 & $0.000 /$ & 0.00 & 6 & $0.017 /$ & -80.01 \\
\hline 18 & 20 & $0.000 /$ & 0.00 & $7.357 /$ & -85.21 & 15 & $5.723 /$ & 95.25 \\
\hline 19 & 21 & $0.876 /$ & -0.42 & $0.000 /$ & 0.00 & 16 & $0.032 /$ & -82.25 \\
\hline 20 & 22 & $0.992 /$ & -0.05 & $0.000 /$ & 0.00 & 17 & $0.000 /$ & -79.32 \\
\hline 21 & 23 & $0.879 /$ & -0.31 & $0.000 /$ & 0.00 & 18 & $0.006 /$ & -83.68 \\
\hline \multirow[t]{3}{*}{22} & 24 & $0.852 /$ & -0.34 & $0.000 /$ & 0.00 & 4 & $0.194 /$ & -84.18 \\
\hline & & & & & & 31 & $0.142 /$ & 94.61 \\
\hline & & & & & & 32 & $0.052 /$ & 99.10 \\
\hline \multirow[t]{2}{*}{23} & 25 & $0.990 /$ & -0.07 & $0.000 /$ & 0.00 & 28 & $0.004 /$ & 104.81 \\
\hline & & & & & & 9 & $0.004 /$ & -75.19 \\
\hline \multirow[t]{2}{*}{24} & 26 & $0.990 /$ & -0.07 & $0.000 /$ & 0.00 & 29 & $0.004 /$ & 104.13 \\
\hline & & & & & & 3 & $0.004 /$ & -75.87 \\
\hline \multirow[t]{3}{*}{25} & 27 & $0.852 /$ & -0.34 & $0.000 /$ & 0.00 & 30 & $0.059 /$ & 99.42 \\
\hline & & & & & & 4 & $0.131 /$ & -80.25 \\
\hline & & & & & & 10 & $0.071 /$ & 100.03 \\
\hline \multirow[t]{3}{*}{26} & 28 & $0.991 /$ & -0.06 & $0.000 /$ & 0.00 & 25 & $0.004 /$ & -75.19 \\
\hline & & & & & & 33 & $0.001 /$ & 101.43 \\
\hline & & & & & & 41 & $0.000 /$ & -121.54 \\
\hline \multirow[t]{4}{*}{27} & 29 & $0.991 /$ & -0.06 & $0.000 /$ & 0.00 & 26 & $0.004 /$ & -75.87 \\
\hline & & & & & & 34 & $0.000 /$ & 104.65 \\
\hline & & & & & & 38 & $0.000 /$ & -133.94 \\
\hline & & & & & & 38 & $0.000 /$ & -133.94 \\
\hline \multirow[t]{2}{*}{28} & 30 & $0.874 /$ & -0.31 & $0.000 /$ & 0.00 & 27 & $0.059 /$ & -80.58 \\
\hline & & & & & & 35 & $0.013 /$ & 97.14 \\
\hline \multirow[t]{2}{*}{29} & 31 & $0.852 /$ & -0.35 & $0.000 /$ & 0.00 & 36 & $0.142 /$ & 94.61 \\
\hline & & & & & & 24 & $0.142 /$ & -85.39 \\
\hline \multirow[t]{2}{*}{30} & 32 & $0.852 /$ & -0.34 & $0.000 /$ & 0.00 & 37 & $0.052 /$ & 99.10 \\
\hline & & & & & & 24 & $0.052 /$ & -80.90 \\
\hline 31 & 33 & $0.991 /$ & -0.06 & $0.000 /$ & 0.00 & 28 & $0.001 /$ & -78.57 \\
\hline 32 & 34 & $0.991 /$ & -0.06 & $0.000 /$ & 0.00 & 29 & $0.000 /$ & -75.35 \\
\hline 33 & 35 & $0.875 /$ & -0.34 & $0.000 /$ & 0.00 & 30 & $0.013 /$ & -82.86 \\
\hline 34 & 36 & $0.884 /$ & -0.38 & $0.000 /$ & 0.00 & 31 & $0.142 /$ & -85.39 \\
\hline 35 & 37 & $0.882 /$ & -0.37 & $0.000 /$ & 0.00 & 32 & $0.052 /$ & -80.90 \\
\hline \multirow[t]{2}{*}{36} & 38 & $0.991 /$ & -0.06 & $0.000 /$ & 0.00 & 29 & $0.000 /$ & 46.06 \\
\hline & & & & & & 29 & $0.000 /$ & 46.06 \\
\hline 37 & 39 & $0.992 /$ & -0.06 & $0.000 /$ & 0.00 & 5 & $0.008 /$ & -80.45 \\
\hline 38 & 41 & $0.991 /$ & -0.06 & $0.000 /$ & 0.00 & 28 & $0.000 /$ & 58.46 \\
\hline 39 & 49 & $0.991 /$ & -0.06 & $0.000 /$ & 0.00 & 5 & $0.003 /$ & -77.56 \\
\hline 40 & 50 & $0.990 /$ & -0.07 & $0.000 /$ & 0.00 & 51 & $0.003 /$ & 103.39 \\
\hline
\end{tabular}




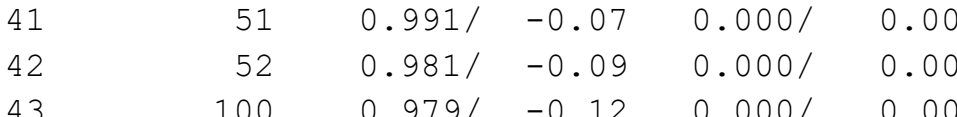

$3 \quad 0.142 /-81.18$

$30.142 /-81.18$

41

$\begin{array}{lll}100 & 0.979 / & -0.12\end{array}$

$0.000 /$

$50 \quad 0.003 /-76.61$

$4 \quad 2.430 /-85.20$

$10.186 / 99.46$

$2 \quad 2.270 /-84.13$

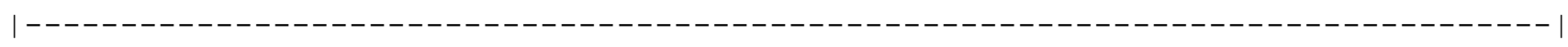




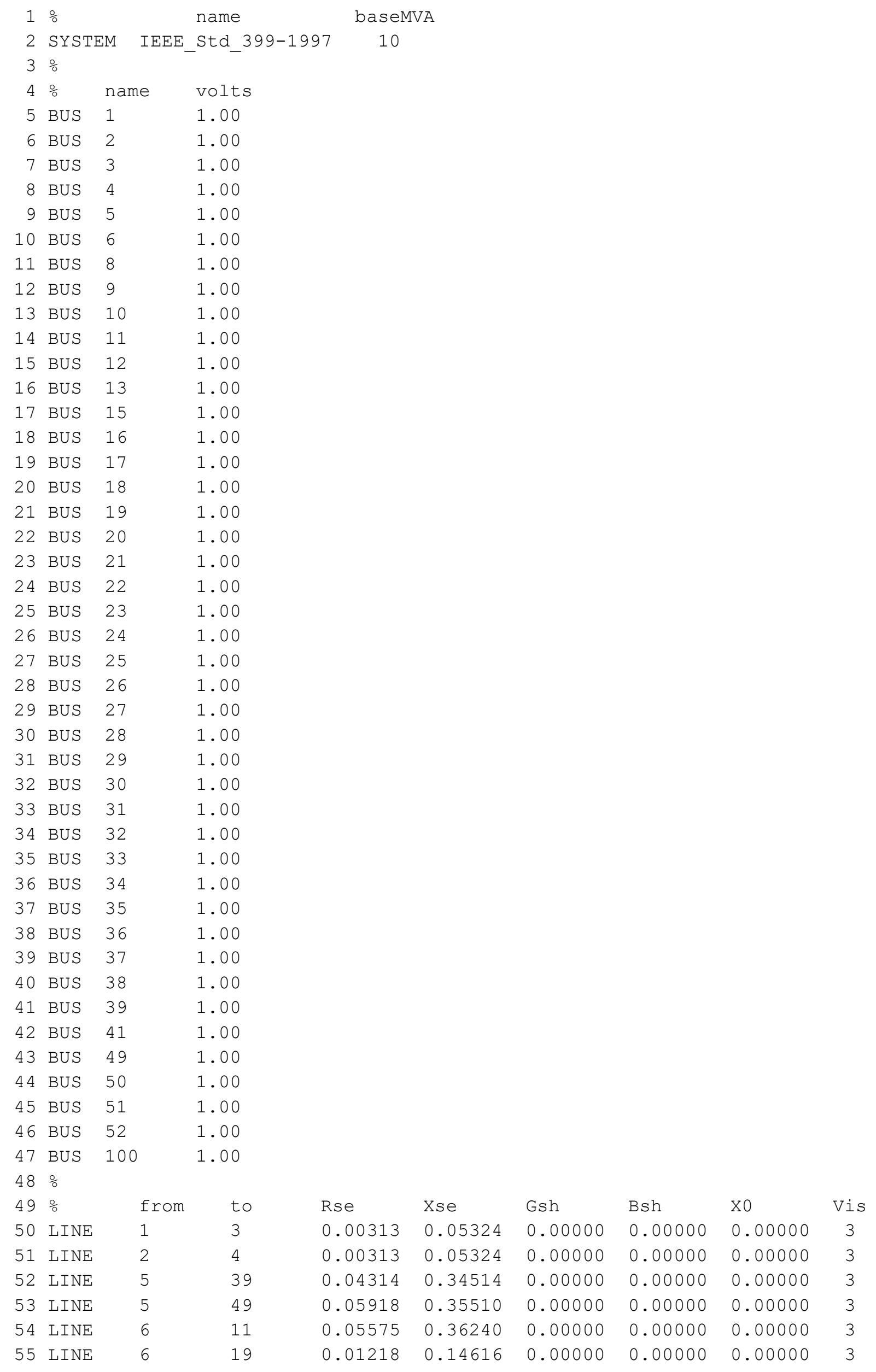




\begin{tabular}{|c|c|c|c|c|c|c|c|c|}
\hline 56 & LINE & 12 & 17 & 0.06843 & 0.44477 & 0.00000 & 0.00000 & 0.00000 \\
\hline 57 & LINE & 13 & 18 & 0.05829 & 0.37888 & 0.00000 & 0.00000 & 0.00000 \\
\hline 58 & LINE & 15 & 20 & 0.01218 & 0.14616 & 0.00000 & 0.00000 & 0.00000 \\
\hline 59 & LINE & 16 & 21 & 0.15036 & 0.75178 & 0.00000 & 0.00000 & 0.00000 \\
\hline 60 & LINE & 25 & 28 & 0.05829 & 0.37888 & 0.00000 & 0.00000 & 0.00000 \\
\hline 61 & LINE & 26 & 29 & 0.05829 & 0.37888 & 0.00000 & 0.00000 & 0.00000 \\
\hline 62 & LINE & 27 & 30 & 0.05829 & 0.37888 & 0.00000 & 0.00000 & 0.00000 \\
\hline 63 & LINE & 31 & 36 & 0.02289 & 0.22886 & 0.00000 & 0.00000 & 0.00000 \\
\hline 64 & LINE & 32 & 37 & 0.10286 & 0.56573 & 0.00000 & 0.00000 & 0.00000 \\
\hline 65 & LINE & 50 & 51 & 0.06395 & 0.37796 & 0.00000 & 0.00000 & 0.00000 \\
\hline 66 & LINE & 52 & 4 & 0.00313 & 0.05324 & 0.00000 & 0.00000 & 0.00000 \\
\hline 67 & LINE & 3 & 5 & 0.00075 & 0.00063 & 0.00000 & 0.00000 & 0.00000 \\
\hline 68 & LINE & 3 & 6 & 0.00109 & 0.00091 & 0.00000 & 0.00000 & 0.00000 \\
\hline 69 & LINE & 3 & 9 & 0.00150 & 0.00125 & 0.00000 & 0.00000 & 0.00000 \\
\hline 70 & LINE & 3 & 26 & 0.00157 & 0.00131 & 0.00000 & 0.00000 & 0.00000 \\
\hline 71 & LINE & 4 & 8 & 0.00076 & 0.00092 & 0.00000 & 0.00000 & 0.00000 \\
\hline 72 & LINE & 4 & 15 & 0.00227 & 0.00189 & 0.00000 & 0.00000 & 0.00000 \\
\hline 73 & LINE & 4 & 24 & 0.00118 & 0.00098 & 0.00000 & 0.00000 & 0.00000 \\
\hline 74 & LINE & 4 & 16 & 0.00274 & 0.00229 & 0.00000 & 0.00000 & 0.00000 \\
\hline 75 & LINE & 4 & 27 & 0.00143 & 0.00119 & 0.00000 & 0.00000 & 0.00000 \\
\hline 76 & LINE & 9 & 12 & 0.00038 & 0.00032 & 0.00000 & 0.00000 & 0.00000 \\
\hline 77 & LINE & 9 & 25 & 0.00424 & 0.00353 & 0.00000 & 0.00000 & 0.00000 \\
\hline 78 & LINE & 10 & 13 & 0.00046 & 0.00039 & 0.00000 & 0.00000 & 0.00000 \\
\hline 79 & LINE & 10 & 27 & 0.00110 & 0.00091 & 0.00000 & 0.00000 & 0.00000 \\
\hline 80 & LINE & 17 & 22 & 0.03813 & 0.02451 & 0.00000 & 0.00000 & 0.00000 \\
\hline 81 & LINE & 18 & 23 & 0.03813 & 0.02451 & 0.00000 & 0.00000 & 0.00000 \\
\hline 82 & LINE & 24 & 31 & 0.00079 & 0.00065 & 0.00000 & 0.00000 & 0.00000 \\
\hline 83 & LINE & 24 & 32 & 0.00112 & 0.00093 & 0.00000 & 0.00000 & 0.00000 \\
\hline 84 & LINE & 28 & 33 & 0.03813 & 0.02451 & 0.00000 & 0.00000 & 0.00000 \\
\hline 85 & LINE & 28 & 41 & 0.03429 & 0.02105 & 0.00000 & 0.00000 & 0.00000 \\
\hline 86 & LINE & 29 & 34 & 0.03813 & 0.02451 & 0.00000 & 0.00000 & 0.00000 \\
\hline 87 & LINE & 29 & 38 & 0.08024 & 0.07732 & 0.00000 & 0.00000 & 0.00000 \\
\hline 88 & LINE & 29 & 38 & 0.08024 & 0.07732 & 0.00000 & 0.00000 & 0.00000 \\
\hline 89 & LINE & 30 & 35 & 0.03813 & 0.02451 & 0.00000 & 0.00000 & 0.00000 \\
\hline 90 & LINE & 50 & 3 & 0.00243 & 0.00485 & 0.00000 & 0.00000 & 0.00000 \\
\hline 91 & LINE & 50 & 3 & 0.00243 & 0.00485 & 0.00000 & 0.00000 & 0.00000 \\
\hline 92 & LINE & 100 & 1 & 0.00139 & 0.00296 & 0.00000 & 0.00000 & 0.00000 \\
\hline 93 & LINE & 100 & 2 & 0.00139 & 0.00296 & 0.00000 & 0.00000 & 0.00000 \\
\hline
\end{tabular}

\begin{tabular}{|c|c|c|c|c|c|c|c|}
\hline $95 \div$ & bus & $\mathrm{R}$ & Xs & $\mathrm{Xp}$ & Xpp & $\mathrm{x} 2$ & xo \\
\hline 96 GENERATOR & 50 & 0.002 & 0.000 & 0.000 & 0.072 & 0.000 & 0.000 \\
\hline 97 GENERATOR & 52 & 0.000 & 0.000 & 0.000 & 0.0062 & 0.000 & 0.000 \\
\hline 98 GENERATOR & 100 & 0.000 & 0.000 & 0.000 & 0.010 & 0.000 & 0.000 \\
\hline $99 \div$ & & & & & & & \\
\hline $100 \%$ & bus & $\mathrm{R}$ & $X s$ & $\mathrm{xp}$ & Xpp & $\mathrm{x} 2$ & xo \\
\hline 101 MOTOR & 11 & 0.352 & 0.000 & 0.000 & 4.219 & 0.000 & 0.000 \\
\hline 102 MOTOR & 17 & 0.338 & 0.000 & 0.000 & 3.384 & 0.000 & 0.000 \\
\hline 103 MOTOR & 17 & 0.802 & 0.000 & 0.000 & 4.008 & 0.000 & 0.000 \\
\hline 104 MOTOR & 18 & 0.338 & 0.000 & 0.000 & 3.384 & 0.000 & 0.000 \\
\hline 105 MOTOR & 18 & 0.802 & 0.000 & 0.000 & 4.008 & 0.000 & 0.000 \\
\hline 106 MOTOR & 19 & 0.057 & 0.000 & 0.000 & 1.484 & 0.000 & 0.000 \\
\hline 107 MOTOR & 19 & 0.047 & 0.000 & 0.000 & 0.703 & 0.000 & 0.000 \\
\hline 108 MOTOR & 20 & 0.067 & 0.000 & 0.000 & 1.005 & 0.000 & 0.000 \\
\hline 109 MOTOR & 20 & 0.060 & 0.000 & 0.000 & 1.556 & 0.000 & 0.000 \\
\hline MOTOR & 21 & 0.320 & 0.000 & 0.000 & 3.835 & 0.000 & 0.000 \\
\hline
\end{tabular}




\begin{tabular}{|c|c|c|c|c|c|c|c|c|c|}
\hline 111 & MOTOR & & 22 & 1.398 & 0.000 & 0.000 & 19.571 & 0.000 & 0.000 \\
\hline 112 & MOTOR & & 23 & 1.398 & 0.000 & 0.000 & 19.571 & 0.000 & 0.000 \\
\hline 113 & MOTOR & & 28 & 0.697 & 0.000 & 0.000 & 6.972 & 0.000 & 0.000 \\
\hline 114 & MOTOR & & 28 & 0.802 & 0.000 & 0.000 & 4.008 & 0.000 & 0.000 \\
\hline 115 & MOTOR & & 29 & 0.321 & 0.000 & 0.000 & 3.206 & 0.000 & 0.000 \\
\hline 116 & MOTOR & & 29 & 1.199 & 0.000 & 0.000 & 5.998 & 0.000 & 0.000 \\
\hline 117 & MOTOR & & 30 & 0.431 & 0.000 & 0.000 & 5.166 & 0.000 & 0.000 \\
\hline 118 & MOTOR & & 30 & 1.116 & 0.000 & 0.000 & 5.578 & 0.000 & 0.000 \\
\hline 119 & MOTOR & & 33 & 0.809 & 0.000 & 0.000 & 9.701 & 0.000 & 0.000 \\
\hline 120 & MOTOR & & 34 & 3.621 & 0.000 & 0.000 & 25.354 & 0.000 & 0.000 \\
\hline 121 & MOTOR & & 35 & 0.809 & 0.000 & 0.000 & 9.701 & 0.000 & 0.000 \\
\hline 122 & MOTOR & & 36 & 0.025 & 0.000 & 0.000 & 0.818 & 0.000 & 0.000 \\
\hline 123 & MOTOR & & 37 & 0.246 & 0.000 & 0.000 & 2.952 & 0.000 & 0.000 \\
\hline 124 & MOTOR & & 37 & 1.859 & 0.000 & 0.000 & 9.296 & 0.000 & 0.000 \\
\hline 125 & MOTOR & & 39 & 0.034 & 0.000 & 0.000 & 1.005 & 0.000 & 0.000 \\
\hline 126 & MOTOR & & 49 & 0.264 & 0.000 & 0.000 & 2.640 & 0.000 & 0.000 \\
\hline 127 & MOTOR & & 51 & 1.432 & 0.000 & 0.000 & 10.020 & 0.000 & 0.000 \\
\hline 128 & MOTOR & & 51 & 0.408 & 0.000 & 0.000 & 4.893 & 0.000 & 0.000 \\
\hline 129 & MOTOR & & 8 & 0.006 & 0.000 & 0.000 & 0.222 & 0.000 & 0.000 \\
\hline 130 & $\%$ & & & & & & & & \\
\hline 131 & \% & bus & Calc & Type & Lc_time & $(\Omega-A]$ & $1-\mathrm{S}$ ibt & nsien & ;2-Transient;3-Steady-state \\
\hline 132 & FAULT & 20 & 3 & & $\overline{1}$ & & & & \\
\hline
\end{tabular}




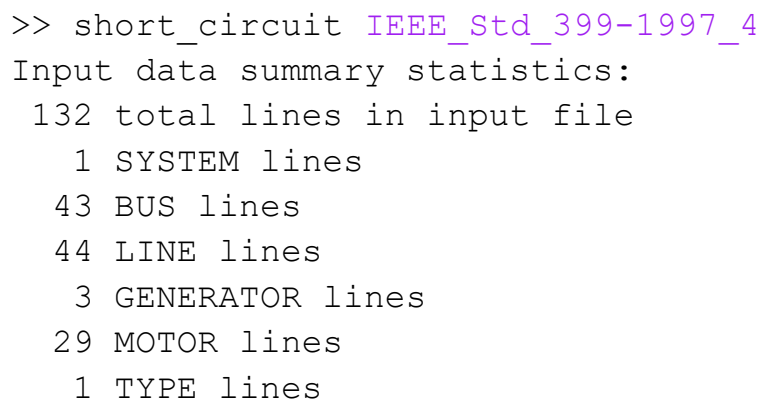

Results for System Name "IEEE_Std_399-1997"

Symmetrical Three-Phase Fault at Bus 20

Calculating Subtransient Currents

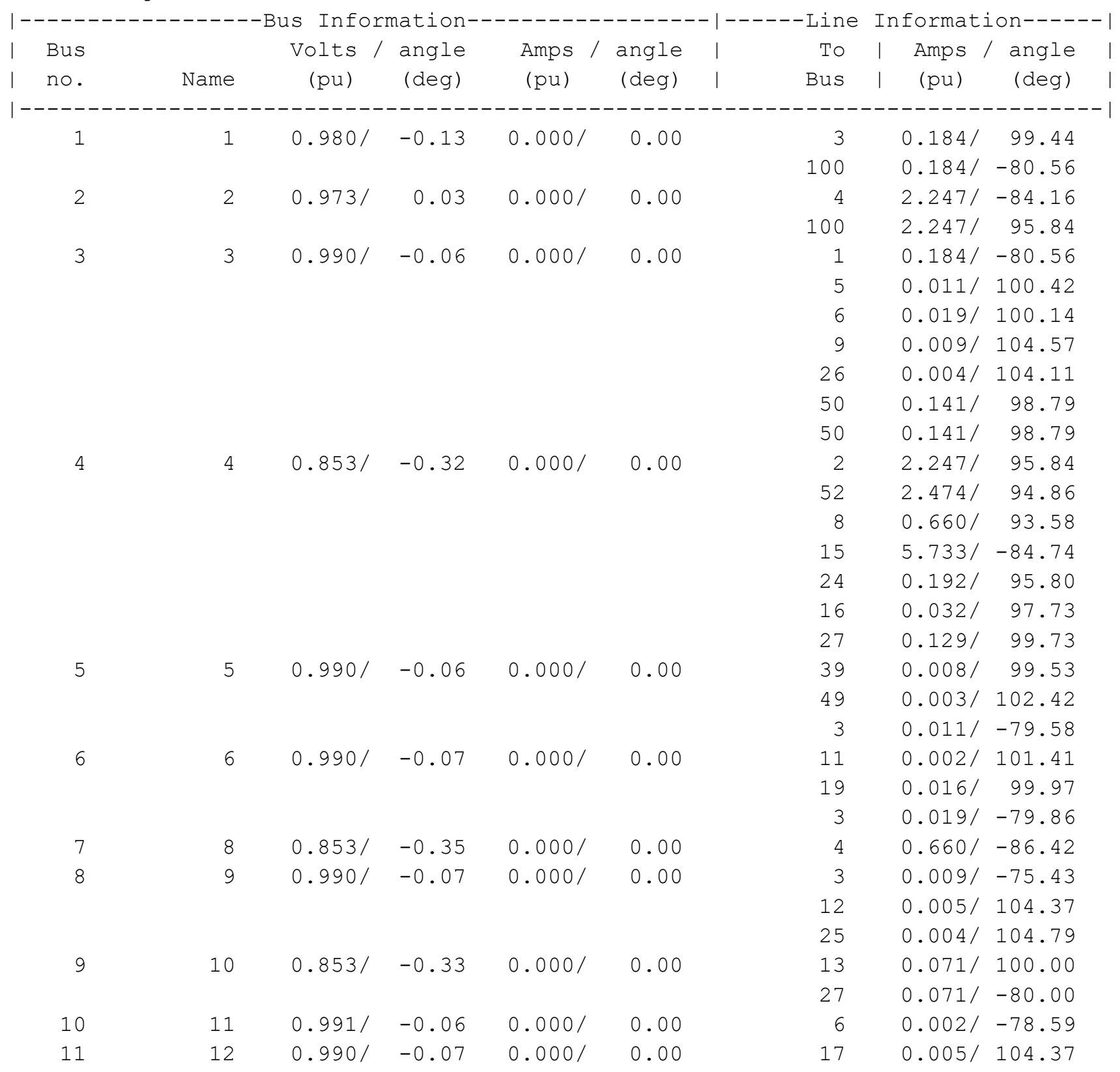




\begin{tabular}{|c|c|c|c|c|c|c|c|c|}
\hline & & & & & & 9 & $0.005 /$ & -75.63 \\
\hline 12 & 13 & $0.853 /$ & -0.34 & $0.000 /$ & 0.00 & 18 & $0.071 /$ & 100.00 \\
\hline & & & & & & 10 & $0.071 /$ & -80.00 \\
\hline 13 & 15 & $0.841 /$ & 0.49 & $0.000 /$ & 0.00 & 20 & $5.733 /$ & -84.74 \\
\hline & & & & & & 4 & $5.733 /$ & 95.26 \\
\hline 14 & 16 & $0.853 /$ & -0.32 & $0.000 /$ & 0.00 & 21 & $0.032 /$ & 97.73 \\
\hline & & & & & & 4 & $0.032 /$ & -82.27 \\
\hline 15 & 17 & $0.992 /$ & -0.05 & $0.000 /$ & 0.00 & 12 & $0.005 /$ & -75.63 \\
\hline & & & & & & 22 & $0.000 /$ & 100.66 \\
\hline 16 & 18 & $0.880 /$ & -0.29 & $0.000 /$ & 0.00 & 13 & $0.071 /$ & -80.00 \\
\hline & & & & & & 23 & $0.006 /$ & 96.29 \\
\hline 17 & 19 & $0.992 /$ & -0.05 & $0.000 /$ & 0.00 & 6 & $0.016 /$ & -80.03 \\
\hline 18 & 20 & $0.000 /$ & 0.00 & $7.367 /$ & -85.20 & 15 & $5.733 /$ & 95.26 \\
\hline 19 & 21 & $0.877 /$ & -0.41 & $0.000 /$ & 0.00 & 16 & $0.032 /$ & -82.27 \\
\hline 20 & 22 & $0.992 /$ & -0.05 & $0.000 /$ & 0.00 & 17 & $0.000 /$ & -79.34 \\
\hline 21 & 23 & $0.880 /$ & -0.30 & $0.000 /$ & 0.00 & 18 & $0.006 /$ & -83.71 \\
\hline 22 & 24 & $0.853 /$ & -0.33 & $0.000 /$ & 0.00 & 4 & $0.192 /$ & -84.20 \\
\hline & & & & & & 31 & $0.140 /$ & 94.59 \\
\hline & & & & & & 32 & $0.052 /$ & 99.08 \\
\hline 23 & 25 & $0.990 /$ & -0.07 & $0.000 /$ & 0.00 & 28 & $0.004 /$ & 104.79 \\
\hline & & & & & & 9 & $0.004 /$ & -75.21 \\
\hline 24 & 26 & $0.990 /$ & -0.06 & $0.000 /$ & 0.00 & 29 & $0.004 /$ & 104.11 \\
\hline & & & & & & 3 & $0.004 /$ & -75.89 \\
\hline 25 & 27 & $0.853 /$ & -0.33 & $0.000 /$ & 0.00 & 30 & $0.059 /$ & 99.39 \\
\hline & & & & & & 4 & $0.129 /$ & -80.27 \\
\hline & & & & & & 10 & $0.071 /$ & 100.00 \\
\hline 26 & 28 & $0.991 /$ & -0.06 & $0.000 /$ & 0.00 & 25 & $0.004 /$ & -75.21 \\
\hline & & & & & & 33 & $0.001 /$ & 101.41 \\
\hline & & & & & & 41 & $0.000 /$ & 148.79 \\
\hline 27 & 29 & $0.991 /$ & -0.06 & $0.000 /$ & 0.00 & 26 & $0.004 /$ & -75.89 \\
\hline & & & & & & 34 & $0.000 /$ & 104.63 \\
\hline & & & & & & 38 & $0.000 /-$ & -133.94 \\
\hline & & & & & & 38 & $0.000 /-$ & -133.94 \\
\hline 28 & 30 & $0.875 /$ & -0.30 & $0.000 /$ & 0.00 & 27 & $0.059 /$ & -80.61 \\
\hline & & & & & & 35 & $0.013 /$ & 97.11 \\
\hline 29 & 31 & $0.853 /$ & -0.34 & $0.000 /$ & 0.00 & 36 & $0.140 /$ & 94.59 \\
\hline & & & & & & 24 & $0.140 /$ & -85.41 \\
\hline 30 & 32 & $0.853 /$ & -0.34 & $0.000 /$ & 0.00 & 37 & $0.052 /$ & 99.08 \\
\hline & & & & & & 24 & $0.052 /$ & -80.92 \\
\hline 31 & 33 & $0.991 /$ & -0.06 & $0.000 /$ & 0.00 & 28 & $0.001 /$ & -78.59 \\
\hline 32 & 34 & $0.991 /$ & -0.06 & $0.000 /$ & 0.00 & 29 & $0.000 /$ & -75.37 \\
\hline 33 & 35 & $0.876 /$ & -0.33 & $0.000 /$ & 0.00 & 30 & $0.013 /$ & -82.89 \\
\hline 34 & 36 & $0.885 /$ & -0.37 & $0.000 /$ & 0.00 & 31 & $0.140 /$ & -85.41 \\
\hline 35 & 37 & $0.883 /$ & -0.37 & $0.000 /$ & 0.00 & 32 & $0.052 /$ & -80.92 \\
\hline 36 & 38 & $0.991 /$ & -0.06 & $0.000 /$ & 0.00 & 29 & $0.000 /$ & 46.06 \\
\hline & & & & & & 29 & $0.000 /$ & 46.06 \\
\hline 37 & 39 & $0.992 /$ & -0.06 & $0.000 /$ & 0.00 & 5 & $0.008 /$ & -80.47 \\
\hline 38 & 41 & $0.991 /$ & -0.06 & $0.000 /$ & 0.00 & 28 & $0.000 /$ & -31.21 \\
\hline 39 & 49 & $0.991 /$ & -0.06 & $0.000 /$ & 0.00 & 5 & $0.003 /$ & -77.58 \\
\hline 40 & 50 & $0.990 /$ & -0.07 & $0.000 /$ & 0.00 & 51 & $0.003 /$ & 103.37 \\
\hline
\end{tabular}




$\begin{array}{lrllll}41 & 51 & 0.991 / & -0.07 & 0.000 / & 0.00 \\ 42 & 52 & 0.985 / & -0.08 & 0.000 / & 0.00 \\ 43 & 100 & 0.979 / & -0.12 & 0.000 / & 0.00\end{array}$

$30.141 /-81.21$

$30.141 /-81.21$

41

43

$0.000 /$

50

$0.003 /-76.63$

$4 \quad 2.474 /-85.14$

$10.184 / 99.44$

$2 \quad 2.247 /-84.16$

$>>$ 


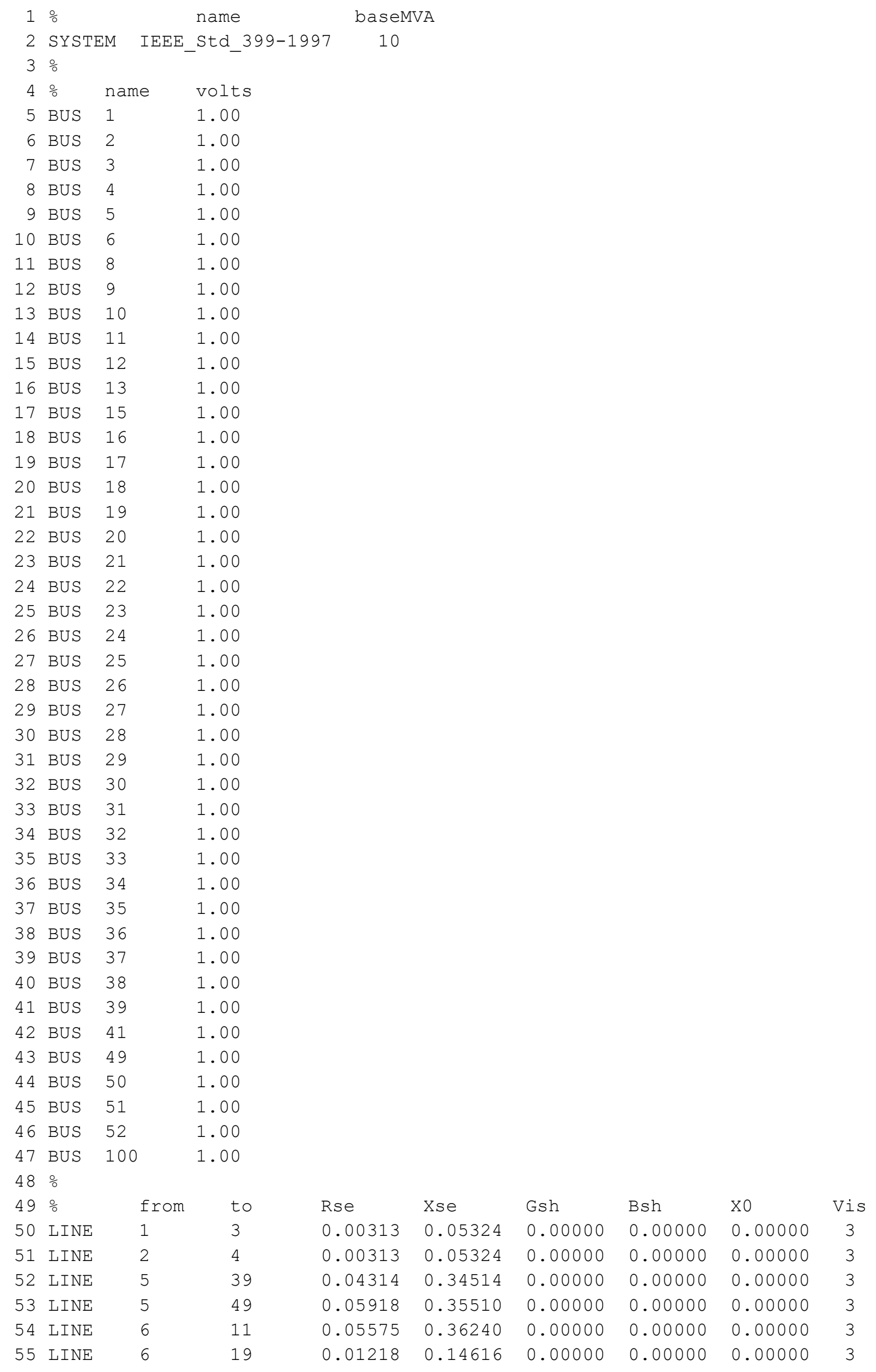




\begin{tabular}{|c|c|c|c|c|c|c|c|c|}
\hline 56 & LINE & 12 & 17 & 0.06843 & 0.44477 & 0.00000 & 0.00000 & 0.00000 \\
\hline 57 & LINE & 13 & 18 & 0.05829 & 0.37888 & 0.00000 & 0.00000 & 0.00000 \\
\hline 58 & LINE & 15 & 20 & 0.01218 & 0.14616 & 0.00000 & 0.00000 & 0.00000 \\
\hline 59 & LINE & 16 & 21 & 0.15036 & 0.75178 & 0.00000 & 0.00000 & 0.00000 \\
\hline 60 & LINE & 25 & 28 & 0.05829 & 0.37888 & 0.00000 & 0.00000 & 0.00000 \\
\hline 61 & LINE & 26 & 29 & 0.05829 & 0.37888 & 0.00000 & 0.00000 & 0.00000 \\
\hline 62 & LINE & 27 & 30 & 0.05829 & 0.37888 & 0.00000 & 0.00000 & 0.00000 \\
\hline 63 & LINE & 31 & 36 & 0.02289 & 0.22886 & 0.00000 & 0.00000 & 0.00000 \\
\hline 64 & LINE & 32 & 37 & 0.10286 & 0.56573 & 0.00000 & 0.00000 & 0.00000 \\
\hline 65 & LINE & 50 & 51 & 0.06395 & 0.37796 & 0.00000 & 0.00000 & 0.00000 \\
\hline 66 & LINE & 52 & 4 & 0.00313 & 0.05324 & 0.00000 & 0.00000 & 0.00000 \\
\hline 67 & LINE & 3 & 5 & 0.00075 & 0.00063 & 0.00000 & 0.00000 & 0.00000 \\
\hline 68 & LINE & 3 & 6 & 0.00109 & 0.00091 & 0.00000 & 0.00000 & 0.00000 \\
\hline 69 & LINE & 3 & 9 & 0.00150 & 0.00125 & 0.00000 & 0.00000 & 0.00000 \\
\hline 70 & LINE & 3 & 26 & 0.00157 & 0.00131 & 0.00000 & 0.00000 & 0.00000 \\
\hline 71 & LINE & 4 & 8 & 0.00076 & 0.00092 & 0.00000 & 0.00000 & 0.00000 \\
\hline 72 & LINE & 4 & 15 & 0.00227 & 0.00189 & 0.00000 & 0.00000 & 0.00000 \\
\hline 73 & LINE & 4 & 24 & 0.00118 & 0.00098 & 0.00000 & 0.00000 & 0.00000 \\
\hline 74 & LINE & 4 & 16 & 0.00274 & 0.00229 & 0.00000 & 0.00000 & 0.00000 \\
\hline 75 & LINE & 4 & 27 & 0.00143 & 0.00119 & 0.00000 & 0.00000 & 0.00000 \\
\hline 76 & LINE & 9 & 12 & 0.00038 & 0.00032 & 0.00000 & 0.00000 & 0.00000 \\
\hline 77 & LINE & 9 & 25 & 0.00424 & 0.00353 & 0.00000 & 0.00000 & 0.00000 \\
\hline 78 & LINE & 10 & 13 & 0.00046 & 0.00039 & 0.00000 & 0.00000 & 0.00000 \\
\hline 79 & LINE & 10 & 27 & 0.00110 & 0.00091 & 0.00000 & 0.00000 & 0.00000 \\
\hline 80 & LINE & 17 & 22 & 0.03813 & 0.02451 & 0.00000 & 0.00000 & 0.00000 \\
\hline 81 & LINE & 18 & 23 & 0.03813 & 0.02451 & 0.00000 & 0.00000 & 0.00000 \\
\hline 82 & LINE & 24 & 31 & 0.00079 & 0.00065 & 0.00000 & 0.00000 & 0.00000 \\
\hline 83 & LINE & 24 & 32 & 0.00112 & 0.00093 & 0.00000 & 0.00000 & 0.00000 \\
\hline 84 & LINE & 28 & 33 & 0.03813 & 0.02451 & 0.00000 & 0.00000 & 0.00000 \\
\hline 85 & LINE & 28 & 41 & 0.03429 & 0.02105 & 0.00000 & 0.00000 & 0.00000 \\
\hline 86 & LINE & 29 & 34 & 0.03813 & 0.02451 & 0.00000 & 0.00000 & 0.00000 \\
\hline 87 & LINE & 29 & 38 & 0.08024 & 0.07732 & 0.00000 & 0.00000 & 0.00000 \\
\hline 88 & LINE & 29 & 38 & 0.08024 & 0.07732 & 0.00000 & 0.00000 & 0.00000 \\
\hline 89 & LINE & 30 & 35 & 0.03813 & 0.02451 & 0.00000 & 0.00000 & 0.00000 \\
\hline 90 & LINE & 50 & 3 & 0.00243 & 0.00485 & 0.00000 & 0.00000 & 0.00000 \\
\hline 91 & LINE & 50 & 3 & 0.00243 & 0.00485 & 0.00000 & 0.00000 & 0.00000 \\
\hline 92 & LINE & 100 & 1 & 0.00139 & 0.00296 & 0.00000 & 0.00000 & 0.00000 \\
\hline 93 & LINE & 100 & 2 & 0.00139 & 0.00296 & 0.00000 & 0.00000 & 0.00000 \\
\hline
\end{tabular}

\begin{tabular}{|c|c|c|c|c|c|c|c|}
\hline $95 \%$ & bus & $\mathrm{R}$ & Xs & $\mathrm{Xp}$ & Xpp & $\mathrm{x} 2$ & xo \\
\hline 96 GENERATOR & 50 & 0.002 & 0.000 & 0.000 & 0.072 & 0.000 & 0.000 \\
\hline 97 GENERATOR & 52 & 0.000 & 0.000 & 0.000 & 0.0056 & 0.000 & 0.000 \\
\hline 98 GENERATOR & 100 & 0.000 & 0.000 & 0.000 & 0.010 & 0.000 & 0.000 \\
\hline $99 \div$ & & & & & & & \\
\hline $100 \%$ & bus & $\mathrm{R}$ & Xs & $\mathrm{xp}$ & Xpp & $\mathrm{x} 2$ & xo \\
\hline 101 MOTOR & 11 & 0.352 & 0.000 & 0.000 & 4.219 & 0.000 & 0.000 \\
\hline 102 MOTOR & 17 & 0.338 & 0.000 & 0.000 & 3.384 & 0.000 & 0.000 \\
\hline 103 MOTOR & 17 & 0.802 & 0.000 & 0.000 & 4.008 & 0.000 & 0.000 \\
\hline 104 MOTOR & 18 & 0.338 & 0.000 & 0.000 & 3.384 & 0.000 & 0.000 \\
\hline 105 MOTOR & 18 & 0.802 & 0.000 & 0.000 & 4.008 & 0.000 & 0.000 \\
\hline 106 MOTOR & 19 & 0.057 & 0.000 & 0.000 & 1.484 & 0.000 & 0.000 \\
\hline 107 MOTOR & 19 & 0.047 & 0.000 & 0.000 & 0.703 & 0.000 & 0.000 \\
\hline 108 MOTOR & 20 & 0.067 & 0.000 & 0.000 & 1.005 & 0.000 & 0.000 \\
\hline 109 MOTOR & 20 & 0.060 & 0.000 & 0.000 & 1.556 & 0.000 & 0.000 \\
\hline MOTOR & 21 & 0.320 & 0.000 & 0.000 & 3.835 & 0.000 & 0.000 \\
\hline
\end{tabular}




\begin{tabular}{|c|c|c|c|c|c|c|c|c|c|}
\hline 111 & MOTOR & & 22 & 1.398 & 0.000 & 0.000 & 19.571 & 0.000 & 0.000 \\
\hline 112 & MOTOR & & 23 & 1.398 & 0.000 & 0.000 & 19.571 & 0.000 & 0.000 \\
\hline 113 & MOTOR & & 28 & 0.697 & 0.000 & 0.000 & 6.972 & 0.000 & 0.000 \\
\hline 114 & MOTOR & & 28 & 0.802 & 0.000 & 0.000 & 4.008 & 0.000 & 0.000 \\
\hline 115 & MOTOR & & 29 & 0.321 & 0.000 & 0.000 & 3.206 & 0.000 & 0.000 \\
\hline 116 & MOTOR & & 29 & 1.199 & 0.000 & 0.000 & 5.998 & 0.000 & 0.000 \\
\hline 117 & MOTOR & & 30 & 0.431 & 0.000 & 0.000 & 5.166 & 0.000 & 0.000 \\
\hline 118 & MOTOR & & 30 & 1.116 & 0.000 & 0.000 & 5.578 & 0.000 & 0.000 \\
\hline 119 & MOTOR & & 33 & 0.809 & 0.000 & 0.000 & 9.701 & 0.000 & 0.000 \\
\hline 120 & MOTOR & & 34 & 3.621 & 0.000 & 0.000 & 25.354 & 0.000 & 0.000 \\
\hline 121 & MOTOR & & 35 & 0.809 & 0.000 & 0.000 & 9.701 & 0.000 & 0.000 \\
\hline 122 & MOTOR & & 36 & 0.025 & 0.000 & 0.000 & 0.818 & 0.000 & 0.000 \\
\hline 123 & MOTOR & & 37 & 0.246 & 0.000 & 0.000 & 2.952 & 0.000 & 0.000 \\
\hline 124 & MOTOR & & 37 & 1.859 & 0.000 & 0.000 & 9.296 & 0.000 & 0.000 \\
\hline 125 & MOTOR & & 39 & 0.034 & 0.000 & 0.000 & 1.005 & 0.000 & 0.000 \\
\hline 126 & MOTOR & & 49 & 0.264 & 0.000 & 0.000 & 2.640 & 0.000 & 0.000 \\
\hline 127 & MOTOR & & 51 & 1.432 & 0.000 & 0.000 & 10.020 & 0.000 & 0.000 \\
\hline 128 & MOTOR & & 51 & 0.408 & 0.000 & 0.000 & 4.893 & 0.000 & 0.000 \\
\hline 129 & MOTOR & & 8 & 0.006 & 0.000 & 0.000 & 0.222 & 0.000 & 0.000 \\
\hline 130 & $\%$ & & & & & & & & \\
\hline 131 & \% & bus & Calc & Type & Lc_time & $(\Omega-A]$ & $1-\mathrm{S}$ ibt & nsien & ;2-Transient;3-Steady-state \\
\hline 132 & FAULT & 20 & 3 & & $\overline{1}$ & & & & \\
\hline
\end{tabular}




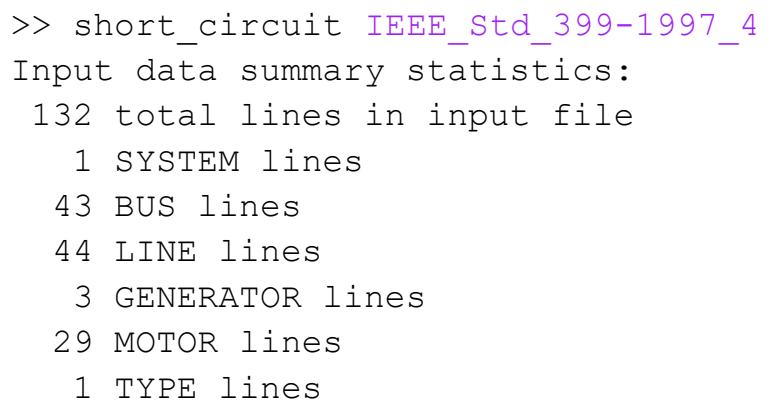

Results for System Name "IEEE_Std_399-1997"

Symmetrical Three-Phase Fault at Bus 20

Calculating Subtransient Currents

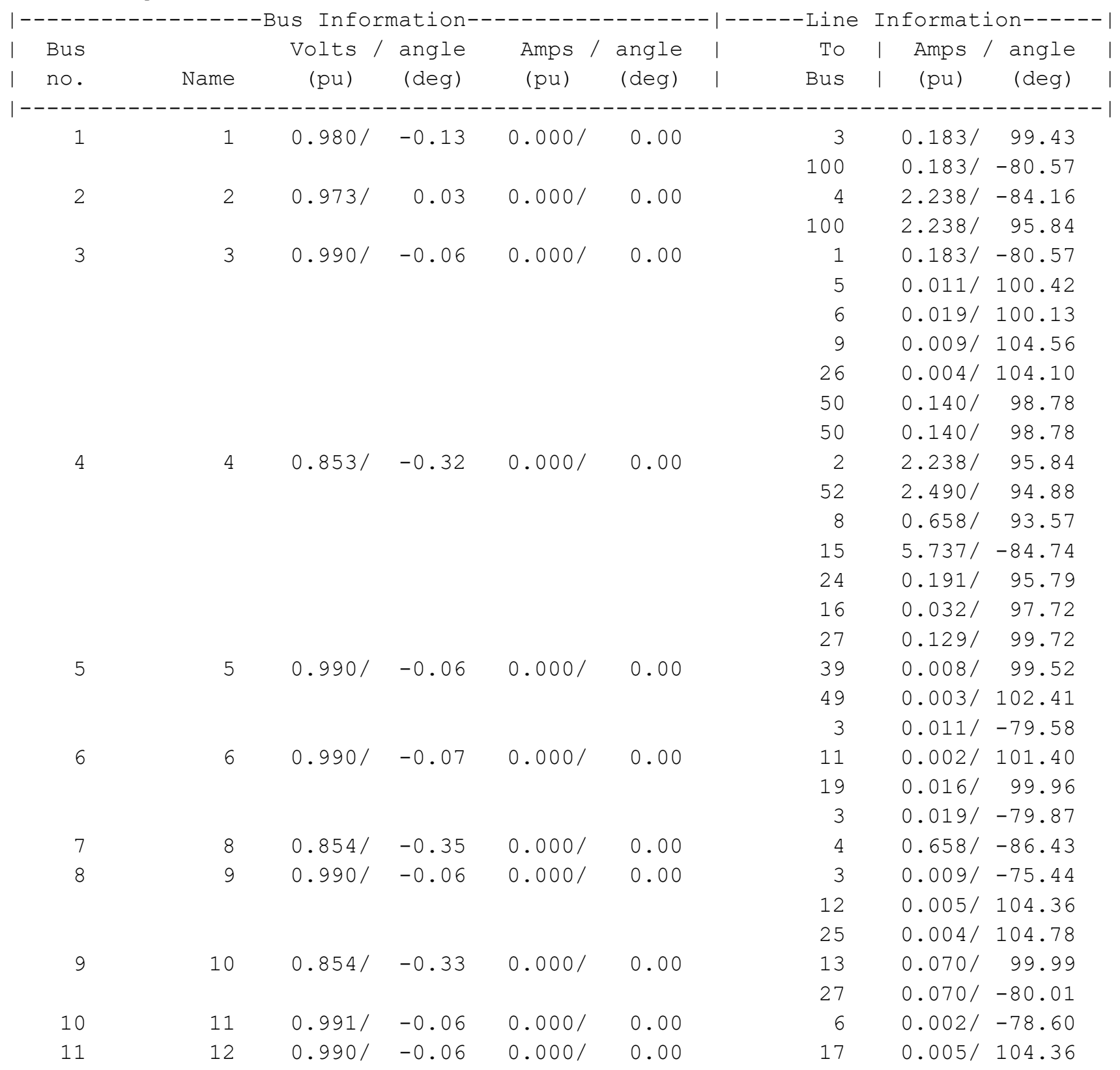




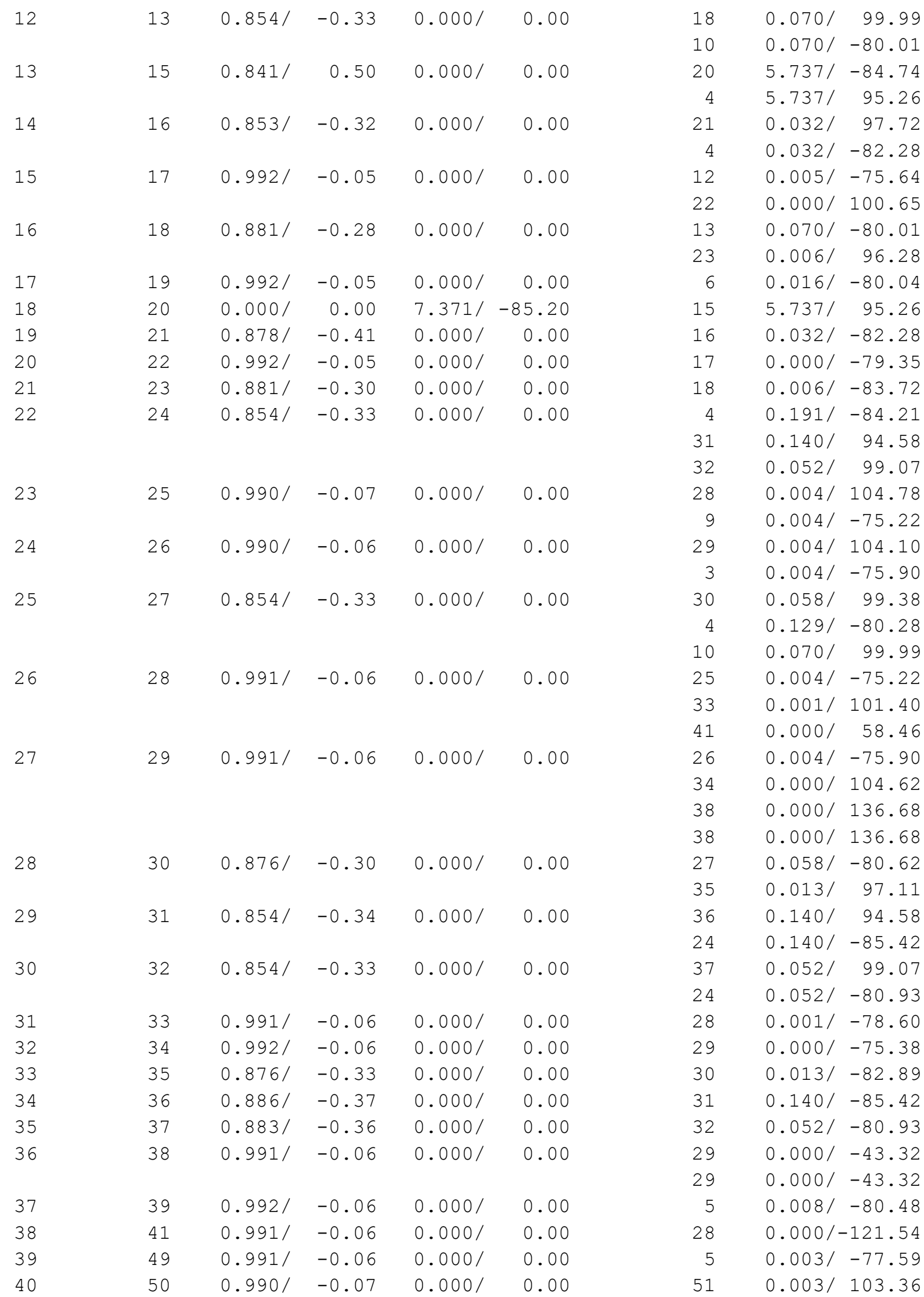




$\begin{array}{lrllll}41 & 51 & 0.991 / & -0.07 & 0.000 / & 0.00 \\ 42 & 52 & 0.986 / & -0.07 & 0.000 / & 0.00 \\ 43 & 100 & 0.980 / & -0.12 & 0.000 / & 0.00\end{array}$

$30.140 /-81.22$

$30.140 /-81.22$

41

43

$0.000 /$

50

$0.003 /-76.64$

$4 \quad 2.490 /-85.12$

$10.183 / 99.43$

$22.238 /-84.16$

$>>$ 


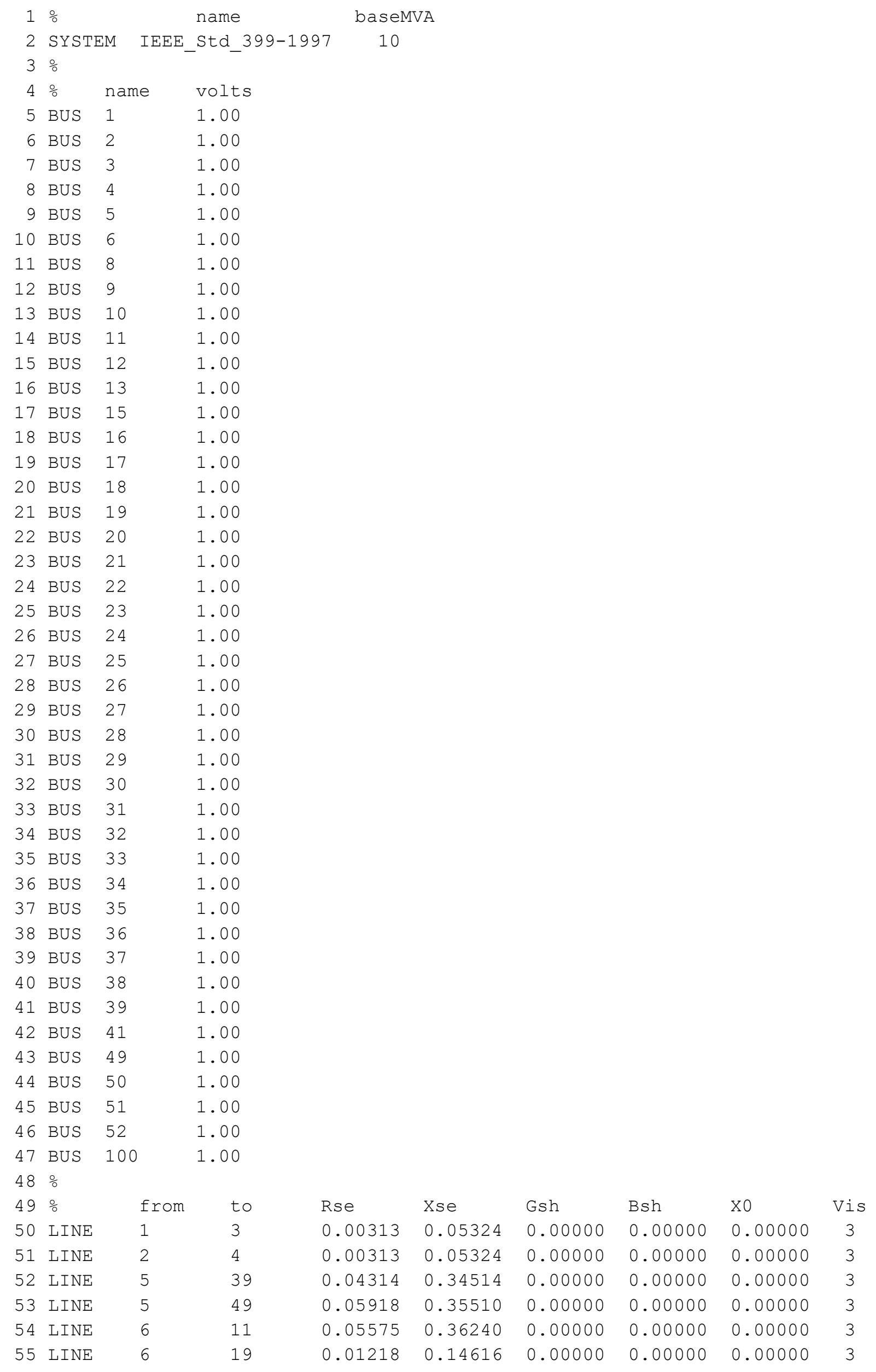




\begin{tabular}{|c|c|c|c|c|c|c|c|c|}
\hline 56 & LINE & 12 & 17 & 0.06843 & 0.44477 & 0.00000 & 0.00000 & 0.00000 \\
\hline 57 & LINE & 13 & 18 & 0.05829 & 0.37888 & 0.00000 & 0.00000 & 0.00000 \\
\hline 58 & LINE & 15 & 20 & 0.01218 & 0.14616 & 0.00000 & 0.00000 & 0.00000 \\
\hline 59 & LINE & 16 & 21 & 0.15036 & 0.75178 & 0.00000 & 0.00000 & 0.00000 \\
\hline 60 & LINE & 25 & 28 & 0.05829 & 0.37888 & 0.00000 & 0.00000 & 0.00000 \\
\hline 61 & LINE & 26 & 29 & 0.05829 & 0.37888 & 0.00000 & 0.00000 & 0.00000 \\
\hline 62 & LINE & 27 & 30 & 0.05829 & 0.37888 & 0.00000 & 0.00000 & 0.00000 \\
\hline 63 & LINE & 31 & 36 & 0.02289 & 0.22886 & 0.00000 & 0.00000 & 0.00000 \\
\hline 64 & LINE & 32 & 37 & 0.10286 & 0.56573 & 0.00000 & 0.00000 & 0.00000 \\
\hline 65 & LINE & 50 & 51 & 0.06395 & 0.37796 & 0.00000 & 0.00000 & 0.00000 \\
\hline 66 & LINE & 52 & 4 & 0.00313 & 0.05324 & 0.00000 & 0.00000 & 0.00000 \\
\hline 67 & LINE & 3 & 5 & 0.00075 & 0.00063 & 0.00000 & 0.00000 & 0.00000 \\
\hline 68 & LINE & 3 & 6 & 0.00109 & 0.00091 & 0.00000 & 0.00000 & 0.00000 \\
\hline 69 & LINE & 3 & 9 & 0.00150 & 0.00125 & 0.00000 & 0.00000 & 0.00000 \\
\hline 70 & LINE & 3 & 26 & 0.00157 & 0.00131 & 0.00000 & 0.00000 & 0.00000 \\
\hline 71 & LINE & 4 & 8 & 0.00076 & 0.00092 & 0.00000 & 0.00000 & 0.00000 \\
\hline 72 & LINE & 4 & 15 & 0.00227 & 0.00189 & 0.00000 & 0.00000 & 0.00000 \\
\hline 73 & LINE & 4 & 24 & 0.00118 & 0.00098 & 0.00000 & 0.00000 & 0.00000 \\
\hline 74 & LINE & 4 & 16 & 0.00274 & 0.00229 & 0.00000 & 0.00000 & 0.00000 \\
\hline 75 & LINE & 4 & 27 & 0.00143 & 0.00119 & 0.00000 & 0.00000 & 0.00000 \\
\hline 76 & LINE & 9 & 12 & 0.00038 & 0.00032 & 0.00000 & 0.00000 & 0.00000 \\
\hline 77 & LINE & 9 & 25 & 0.00424 & 0.00353 & 0.00000 & 0.00000 & 0.00000 \\
\hline 78 & LINE & 10 & 13 & 0.00046 & 0.00039 & 0.00000 & 0.00000 & 0.00000 \\
\hline 79 & LINE & 10 & 27 & 0.00110 & 0.00091 & 0.00000 & 0.00000 & 0.00000 \\
\hline 80 & LINE & 17 & 22 & 0.03813 & 0.02451 & 0.00000 & 0.00000 & 0.00000 \\
\hline 81 & LINE & 18 & 23 & 0.03813 & 0.02451 & 0.00000 & 0.00000 & 0.00000 \\
\hline 82 & LINE & 24 & 31 & 0.00079 & 0.00065 & 0.00000 & 0.00000 & 0.00000 \\
\hline 83 & LINE & 24 & 32 & 0.00112 & 0.00093 & 0.00000 & 0.00000 & 0.00000 \\
\hline 84 & LINE & 28 & 33 & 0.03813 & 0.02451 & 0.00000 & 0.00000 & 0.00000 \\
\hline 85 & LINE & 28 & 41 & 0.03429 & 0.02105 & 0.00000 & 0.00000 & 0.00000 \\
\hline 86 & LINE & 29 & 34 & 0.03813 & 0.02451 & 0.00000 & 0.00000 & 0.00000 \\
\hline 87 & LINE & 29 & 38 & 0.08024 & 0.07732 & 0.00000 & 0.00000 & 0.00000 \\
\hline 88 & LINE & 29 & 38 & 0.08024 & 0.07732 & 0.00000 & 0.00000 & 0.00000 \\
\hline 89 & LINE & 30 & 35 & 0.03813 & 0.02451 & 0.00000 & 0.00000 & 0.00000 \\
\hline 90 & LINE & 50 & 3 & 0.00243 & 0.00485 & 0.00000 & 0.00000 & 0.00000 \\
\hline 91 & LINE & 50 & 3 & 0.00243 & 0.00485 & 0.00000 & 0.00000 & 0.00000 \\
\hline 92 & LINE & 100 & 1 & 0.00139 & 0.00296 & 0.00000 & 0.00000 & 0.00000 \\
\hline 93 & LINE & 100 & 2 & 0.00139 & 0.00296 & 0.00000 & 0.00000 & 0.00000 \\
\hline
\end{tabular}

\begin{tabular}{|c|c|c|c|c|c|c|c|}
\hline $95 \div$ & bus & $\mathrm{R}$ & Xs & $\mathrm{Xp}$ & Xpp & $\mathrm{x} 2$ & xo \\
\hline 96 GENERATOR & 50 & 0.002 & 0.000 & 0.000 & 0.072 & 0.000 & 0.000 \\
\hline 97 GENERATOR & 52 & 0.000 & 0.000 & 0.000 & 0.0054 & 0.000 & 0.000 \\
\hline 98 GENERATOR & 100 & 0.000 & 0.000 & 0.000 & 0.010 & 0.000 & 0.000 \\
\hline $99 \div$ & & & & & & & \\
\hline $100 \%$ & bus & $\mathrm{R}$ & $X s$ & $\mathrm{xp}$ & Xpp & $\mathrm{x} 2$ & xo \\
\hline 101 MOTOR & 11 & 0.352 & 0.000 & 0.000 & 4.219 & 0.000 & 0.000 \\
\hline 102 MOTOR & 17 & 0.338 & 0.000 & 0.000 & 3.384 & 0.000 & 0.000 \\
\hline 103 MOTOR & 17 & 0.802 & 0.000 & 0.000 & 4.008 & 0.000 & 0.000 \\
\hline 104 MOTOR & 18 & 0.338 & 0.000 & 0.000 & 3.384 & 0.000 & 0.000 \\
\hline 105 MOTOR & 18 & 0.802 & 0.000 & 0.000 & 4.008 & 0.000 & 0.000 \\
\hline 106 MOTOR & 19 & 0.057 & 0.000 & 0.000 & 1.484 & 0.000 & 0.000 \\
\hline 107 MOTOR & 19 & 0.047 & 0.000 & 0.000 & 0.703 & 0.000 & 0.000 \\
\hline 108 MOTOR & 20 & 0.067 & 0.000 & 0.000 & 1.005 & 0.000 & 0.000 \\
\hline 109 MOTOR & 20 & 0.060 & 0.000 & 0.000 & 1.556 & 0.000 & 0.000 \\
\hline MOTOR & 21 & 0.320 & 0.000 & 0.000 & 3.835 & 0.000 & 0.000 \\
\hline
\end{tabular}




\begin{tabular}{|c|c|c|c|c|c|c|c|c|c|}
\hline 111 & MOTOR & & 22 & 1.398 & 0.000 & 0.000 & 19.571 & 0.000 & 0.000 \\
\hline 112 & MOTOR & & 23 & 1.398 & 0.000 & 0.000 & 19.571 & 0.000 & 0.000 \\
\hline 113 & MOTOR & & 28 & 0.697 & 0.000 & 0.000 & 6.972 & 0.000 & 0.000 \\
\hline 114 & MOTOR & & 28 & 0.802 & 0.000 & 0.000 & 4.008 & 0.000 & 0.000 \\
\hline 115 & MOTOR & & 29 & 0.321 & 0.000 & 0.000 & 3.206 & 0.000 & 0.000 \\
\hline 116 & MOTOR & & 29 & 1.199 & 0.000 & 0.000 & 5.998 & 0.000 & 0.000 \\
\hline 117 & MOTOR & & 30 & 0.431 & 0.000 & 0.000 & 5.166 & 0.000 & 0.000 \\
\hline 118 & MOTOR & & 30 & 1.116 & 0.000 & 0.000 & 5.578 & 0.000 & 0.000 \\
\hline 119 & MOTOR & & 33 & 0.809 & 0.000 & 0.000 & 9.701 & 0.000 & 0.000 \\
\hline 120 & MOTOR & & 34 & 3.621 & 0.000 & 0.000 & 25.354 & 0.000 & 0.000 \\
\hline 121 & MOTOR & & 35 & 0.809 & 0.000 & 0.000 & 9.701 & 0.000 & 0.000 \\
\hline 122 & MOTOR & & 36 & 0.025 & 0.000 & 0.000 & 0.818 & 0.000 & 0.000 \\
\hline 123 & MOTOR & & 37 & 0.246 & 0.000 & 0.000 & 2.952 & 0.000 & 0.000 \\
\hline 124 & MOTOR & & 37 & 1.859 & 0.000 & 0.000 & 9.296 & 0.000 & 0.000 \\
\hline 125 & MOTOR & & 39 & 0.034 & 0.000 & 0.000 & 1.005 & 0.000 & 0.000 \\
\hline 126 & MOTOR & & 49 & 0.264 & 0.000 & 0.000 & 2.640 & 0.000 & 0.000 \\
\hline 127 & MOTOR & & 51 & 1.432 & 0.000 & 0.000 & 10.020 & 0.000 & 0.000 \\
\hline 128 & MOTOR & & 51 & 0.408 & 0.000 & 0.000 & 4.893 & 0.000 & 0.000 \\
\hline 129 & MOTOR & & 8 & 0.006 & 0.000 & 0.000 & 0.222 & 0.000 & 0.000 \\
\hline 130 & $\%$ & & & & & & & & \\
\hline 131 & \% & bus & Calc & Type & Lc_time & $(\Omega-A]$ & $1-\mathrm{S}$ ibt & nsien & ;2-Transient;3-Steady-state \\
\hline 132 & FAULT & 20 & 3 & & $\overline{1}$ & & & & \\
\hline
\end{tabular}




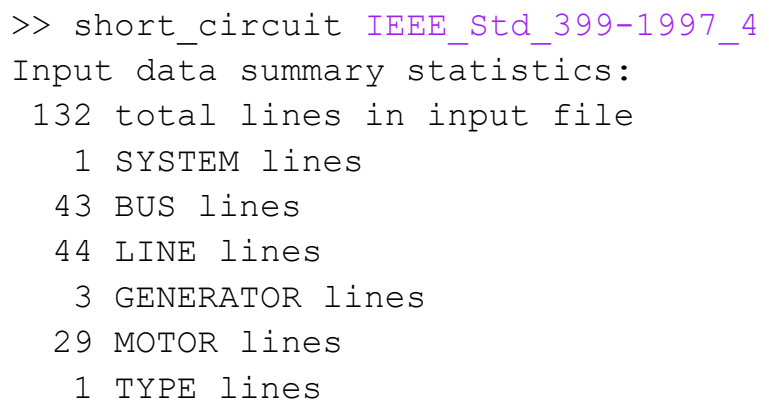

Results for System Name "IEEE_Std_399-1997"

Symmetrical Three-Phase Fault at Bus 20

Calculating Subtransient Currents

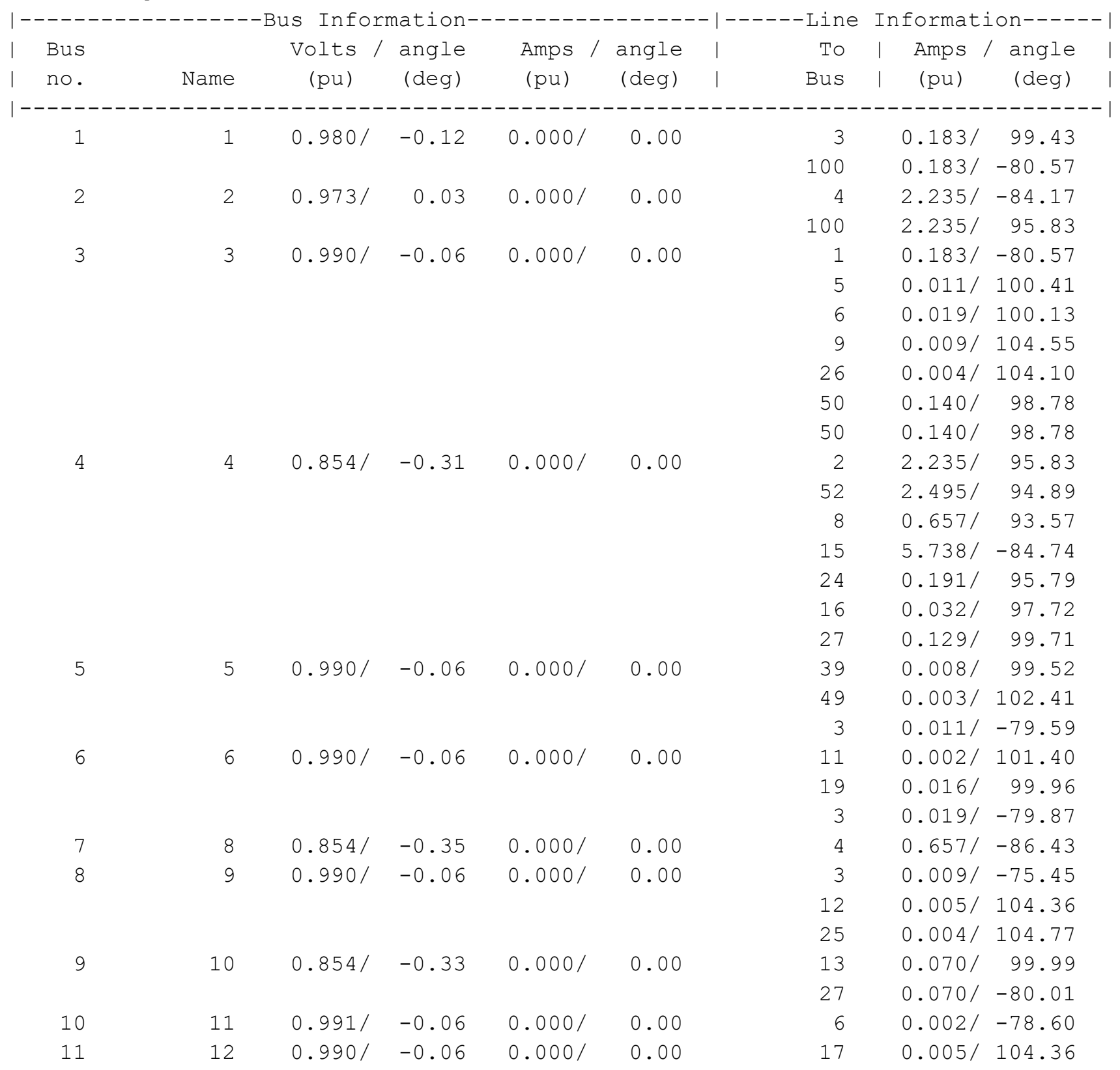




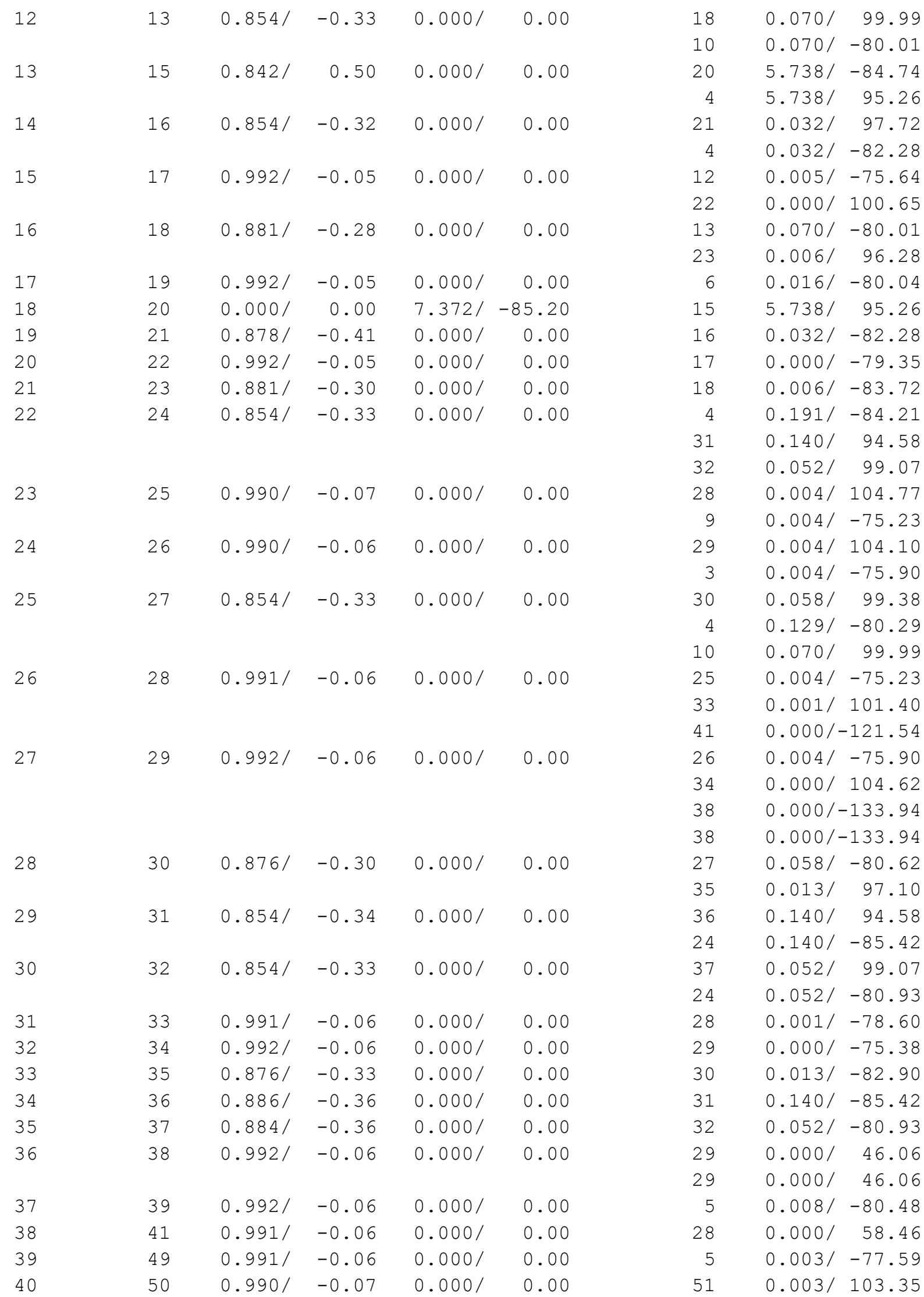




$\begin{array}{rrrrrr}42 & 52 & 0.987 / & -0.07 & 0.000 / & 0.00 \\ 43 & 100 & 0.980 / & -0.12 & 0.000 / & 0.00\end{array}$

$30.140 /-81.22$

$30.140 /-81.22$

41

43

$0.000 /$

50

$0.003 /-76.65$

$4 \quad 2.495 /-85.11$

$10.183 / 99.43$

$2 \quad 2.235 /-84.17$

$>>$ 


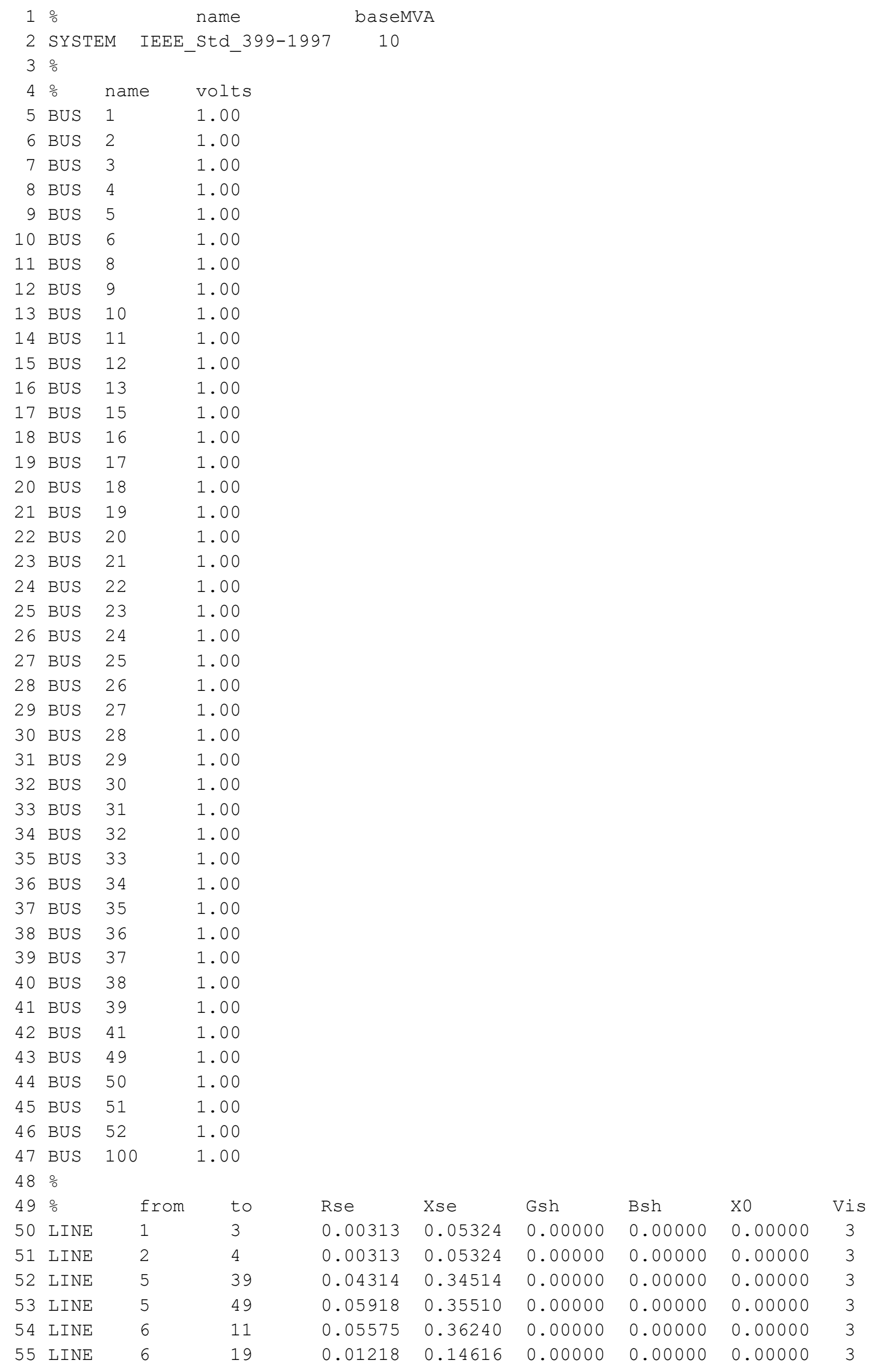




\begin{tabular}{|c|c|c|c|c|c|c|c|c|}
\hline 56 & LINE & 12 & 17 & 0.06843 & 0.44477 & 0.00000 & 0.00000 & 0.00000 \\
\hline 57 & LINE & 13 & 18 & 0.05829 & 0.37888 & 0.00000 & 0.00000 & 0.00000 \\
\hline 58 & LINE & 15 & 20 & 0.01218 & 0.14616 & 0.00000 & 0.00000 & 0.00000 \\
\hline 59 & LINE & 16 & 21 & 0.15036 & 0.75178 & 0.00000 & 0.00000 & 0.00000 \\
\hline 60 & LINE & 25 & 28 & 0.05829 & 0.37888 & 0.00000 & 0.00000 & 0.00000 \\
\hline 61 & LINE & 26 & 29 & 0.05829 & 0.37888 & 0.00000 & 0.00000 & 0.00000 \\
\hline 62 & LINE & 27 & 30 & 0.05829 & 0.37888 & 0.00000 & 0.00000 & 0.00000 \\
\hline 63 & LINE & 31 & 36 & 0.02289 & 0.22886 & 0.00000 & 0.00000 & 0.00000 \\
\hline 64 & LINE & 32 & 37 & 0.10286 & 0.56573 & 0.00000 & 0.00000 & 0.00000 \\
\hline 65 & LINE & 50 & 51 & 0.06395 & 0.37796 & 0.00000 & 0.00000 & 0.00000 \\
\hline 66 & LINE & 52 & 4 & 0.00313 & 0.05324 & 0.00000 & 0.00000 & 0.00000 \\
\hline 67 & LINE & 3 & 5 & 0.00075 & 0.00063 & 0.00000 & 0.00000 & 0.00000 \\
\hline 68 & LINE & 3 & 6 & 0.00109 & 0.00091 & 0.00000 & 0.00000 & 0.00000 \\
\hline 69 & LINE & 3 & 9 & 0.00150 & 0.00125 & 0.00000 & 0.00000 & 0.00000 \\
\hline 70 & LINE & 3 & 26 & 0.00157 & 0.00131 & 0.00000 & 0.00000 & 0.00000 \\
\hline 71 & LINE & 4 & 8 & 0.00076 & 0.00092 & 0.00000 & 0.00000 & 0.00000 \\
\hline 72 & LINE & 4 & 15 & 0.00227 & 0.00189 & 0.00000 & 0.00000 & 0.00000 \\
\hline 73 & LINE & 4 & 24 & 0.00118 & 0.00098 & 0.00000 & 0.00000 & 0.00000 \\
\hline 74 & LINE & 4 & 16 & 0.00274 & 0.00229 & 0.00000 & 0.00000 & 0.00000 \\
\hline 75 & LINE & 4 & 27 & 0.00143 & 0.00119 & 0.00000 & 0.00000 & 0.00000 \\
\hline 76 & LINE & 9 & 12 & 0.00038 & 0.00032 & 0.00000 & 0.00000 & 0.00000 \\
\hline 77 & LINE & 9 & 25 & 0.00424 & 0.00353 & 0.00000 & 0.00000 & 0.00000 \\
\hline 78 & LINE & 10 & 13 & 0.00046 & 0.00039 & 0.00000 & 0.00000 & 0.00000 \\
\hline 79 & LINE & 10 & 27 & 0.00110 & 0.00091 & 0.00000 & 0.00000 & 0.00000 \\
\hline 80 & LINE & 17 & 22 & 0.03813 & 0.02451 & 0.00000 & 0.00000 & 0.00000 \\
\hline 81 & LINE & 18 & 23 & 0.03813 & 0.02451 & 0.00000 & 0.00000 & 0.00000 \\
\hline 82 & LINE & 24 & 31 & 0.00079 & 0.00065 & 0.00000 & 0.00000 & 0.00000 \\
\hline 83 & LINE & 24 & 32 & 0.00112 & 0.00093 & 0.00000 & 0.00000 & 0.00000 \\
\hline 84 & LINE & 28 & 33 & 0.03813 & 0.02451 & 0.00000 & 0.00000 & 0.00000 \\
\hline 85 & LINE & 28 & 41 & 0.03429 & 0.02105 & 0.00000 & 0.00000 & 0.00000 \\
\hline 86 & LINE & 29 & 34 & 0.03813 & 0.02451 & 0.00000 & 0.00000 & 0.00000 \\
\hline 87 & LINE & 29 & 38 & 0.08024 & 0.07732 & 0.00000 & 0.00000 & 0.00000 \\
\hline 88 & LINE & 29 & 38 & 0.08024 & 0.07732 & 0.00000 & 0.00000 & 0.00000 \\
\hline 89 & LINE & 30 & 35 & 0.03813 & 0.02451 & 0.00000 & 0.00000 & 0.00000 \\
\hline 90 & LINE & 50 & 3 & 0.00243 & 0.00485 & 0.00000 & 0.00000 & 0.00000 \\
\hline 91 & LINE & 50 & 3 & 0.00243 & 0.00485 & 0.00000 & 0.00000 & 0.00000 \\
\hline 92 & LINE & 100 & 1 & 0.00139 & 0.00296 & 0.00000 & 0.00000 & 0.00000 \\
\hline 93 & LINE & 100 & 2 & 0.00139 & 0.00296 & 0.00000 & 0.00000 & 0.00000 \\
\hline
\end{tabular}

\begin{tabular}{|c|c|c|c|c|c|c|c|}
\hline $95 \div$ & bus & $\mathrm{R}$ & Xs & $\mathrm{Xp}$ & Xpp & $\mathrm{x} 2$ & xo \\
\hline 96 GENERATOR & 50 & 0.002 & 0.000 & 0.000 & 0.072 & 0.000 & 0.000 \\
\hline 97 GENERATOR & 52 & 0.000 & 0.000 & 0.000 & 0.0052 & 0.000 & 0.000 \\
\hline 98 GENERATOR & 100 & 0.000 & 0.000 & 0.000 & 0.010 & 0.000 & 0.000 \\
\hline $99 \div$ & & & & & & & \\
\hline $100 \%$ & bus & $\mathrm{R}$ & $X s$ & $\mathrm{xp}$ & Xpp & $\mathrm{x} 2$ & xo \\
\hline 101 MOTOR & 11 & 0.352 & 0.000 & 0.000 & 4.219 & 0.000 & 0.000 \\
\hline 102 MOTOR & 17 & 0.338 & 0.000 & 0.000 & 3.384 & 0.000 & 0.000 \\
\hline 103 MOTOR & 17 & 0.802 & 0.000 & 0.000 & 4.008 & 0.000 & 0.000 \\
\hline 104 MOTOR & 18 & 0.338 & 0.000 & 0.000 & 3.384 & 0.000 & 0.000 \\
\hline 105 MOTOR & 18 & 0.802 & 0.000 & 0.000 & 4.008 & 0.000 & 0.000 \\
\hline 106 MOTOR & 19 & 0.057 & 0.000 & 0.000 & 1.484 & 0.000 & 0.000 \\
\hline 107 MOTOR & 19 & 0.047 & 0.000 & 0.000 & 0.703 & 0.000 & 0.000 \\
\hline 108 MOTOR & 20 & 0.067 & 0.000 & 0.000 & 1.005 & 0.000 & 0.000 \\
\hline 109 MOTOR & 20 & 0.060 & 0.000 & 0.000 & 1.556 & 0.000 & 0.000 \\
\hline MOTOR & 21 & 0.320 & 0.000 & 0.000 & 3.835 & 0.000 & 0.000 \\
\hline
\end{tabular}




\begin{tabular}{|c|c|c|c|c|c|c|c|c|c|}
\hline 111 & MOTOR & & 22 & 1.398 & 0.000 & 0.000 & 19.571 & 0.000 & 0.000 \\
\hline 112 & MOTOR & & 23 & 1.398 & 0.000 & 0.000 & 19.571 & 0.000 & 0.000 \\
\hline 113 & MOTOR & & 28 & 0.697 & 0.000 & 0.000 & 6.972 & 0.000 & 0.000 \\
\hline 114 & MOTOR & & 28 & 0.802 & 0.000 & 0.000 & 4.008 & 0.000 & 0.000 \\
\hline 115 & MOTOR & & 29 & 0.321 & 0.000 & 0.000 & 3.206 & 0.000 & 0.000 \\
\hline 116 & MOTOR & & 29 & 1.199 & 0.000 & 0.000 & 5.998 & 0.000 & 0.000 \\
\hline 117 & MOTOR & & 30 & 0.431 & 0.000 & 0.000 & 5.166 & 0.000 & 0.000 \\
\hline 118 & MOTOR & & 30 & 1.116 & 0.000 & 0.000 & 5.578 & 0.000 & 0.000 \\
\hline 119 & MOTOR & & 33 & 0.809 & 0.000 & 0.000 & 9.701 & 0.000 & 0.000 \\
\hline 120 & MOTOR & & 34 & 3.621 & 0.000 & 0.000 & 25.354 & 0.000 & 0.000 \\
\hline 121 & MOTOR & & 35 & 0.809 & 0.000 & 0.000 & 9.701 & 0.000 & 0.000 \\
\hline 122 & MOTOR & & 36 & 0.025 & 0.000 & 0.000 & 0.818 & 0.000 & 0.000 \\
\hline 123 & MOTOR & & 37 & 0.246 & 0.000 & 0.000 & 2.952 & 0.000 & 0.000 \\
\hline 124 & MOTOR & & 37 & 1.859 & 0.000 & 0.000 & 9.296 & 0.000 & 0.000 \\
\hline 125 & MOTOR & & 39 & 0.034 & 0.000 & 0.000 & 1.005 & 0.000 & 0.000 \\
\hline 126 & MOTOR & & 49 & 0.264 & 0.000 & 0.000 & 2.640 & 0.000 & 0.000 \\
\hline 127 & MOTOR & & 51 & 1.432 & 0.000 & 0.000 & 10.020 & 0.000 & 0.000 \\
\hline 128 & MOTOR & & 51 & 0.408 & 0.000 & 0.000 & 4.893 & 0.000 & 0.000 \\
\hline 129 & MOTOR & & 8 & 0.006 & 0.000 & 0.000 & 0.222 & 0.000 & 0.000 \\
\hline 130 & $\%$ & & & & & & & & \\
\hline 131 & \% & bus & Calc & Type & Lc_time & $(\Omega-A]$ & $1-\mathrm{S}$ ibt & nsien & ;2-Transient;3-Steady-state \\
\hline 132 & FAULT & 20 & 3 & & $\overline{1}$ & & & & \\
\hline
\end{tabular}




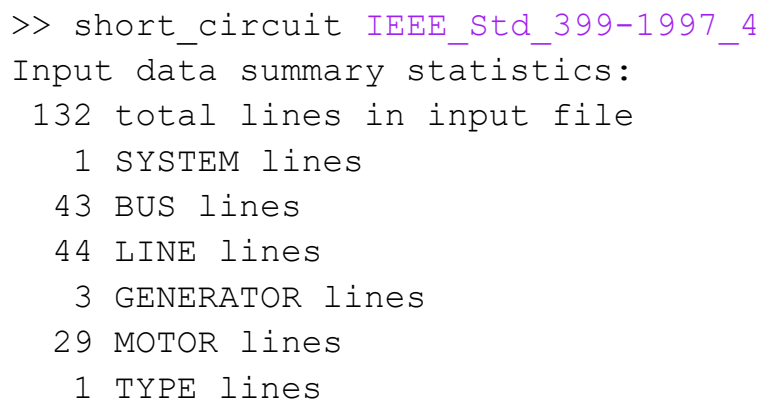

Results for System Name "IEEE_Std_399-1997"

Symmetrical Three-Phase Fault at Bus 20

Calculating Subtransient Currents

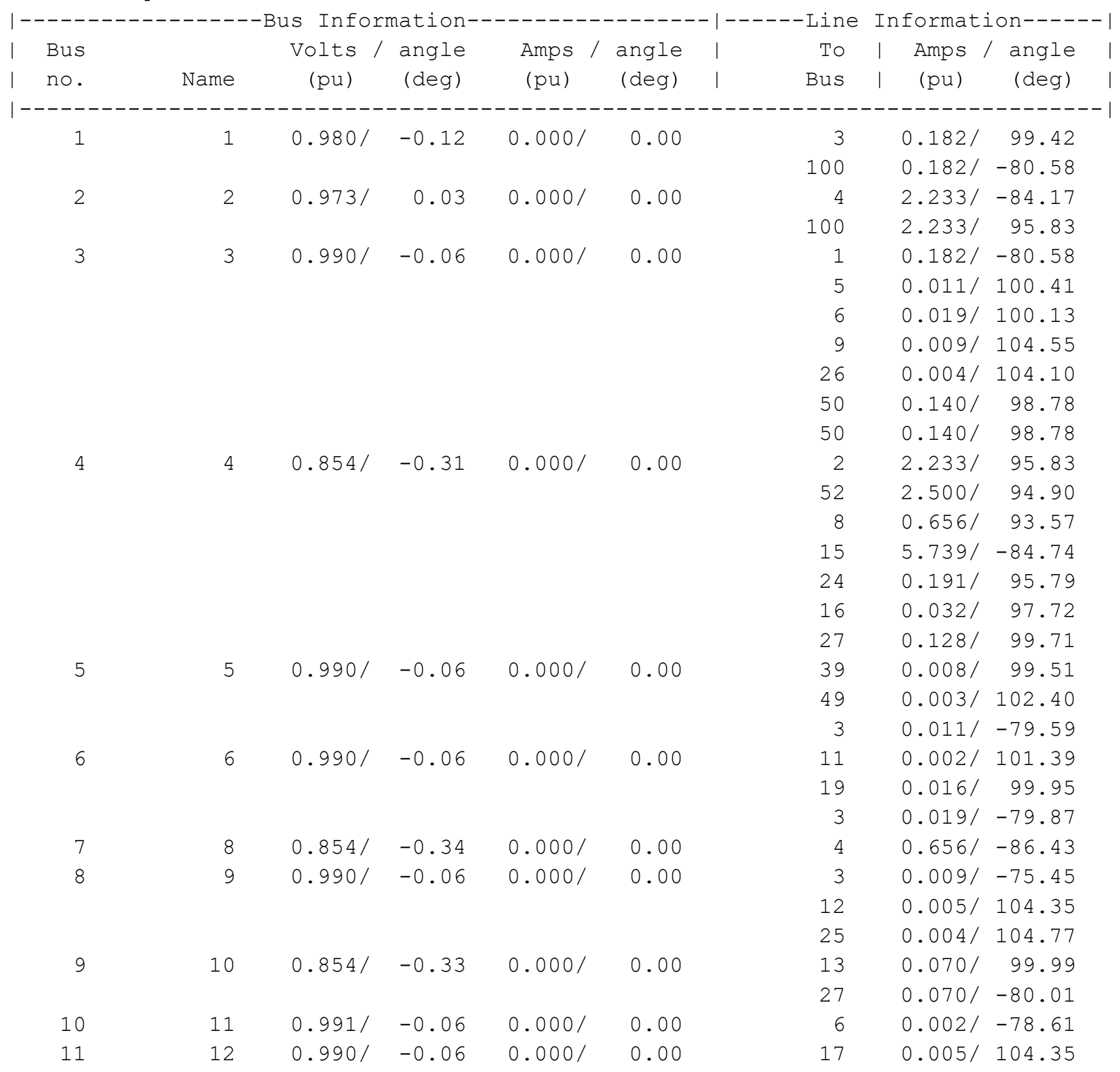




\begin{tabular}{|c|c|c|c|c|c|c|c|c|}
\hline 12 & 13 & $0.854 /$ & -0.33 & $0.000 /$ & 0.00 & 18 & $0.070 /$ & 99.99 \\
\hline & & & & & & 10 & $0.070 /$ & -80.01 \\
\hline 13 & 15 & $0.842 /$ & 0.50 & $0.000 /$ & 0.00 & 20 & $5.739 /$ & -84.74 \\
\hline & & & & & & 4 & $5.739 /$ & 95.26 \\
\hline 14 & 16 & $0.854 /$ & -0.32 & $0.000 /$ & 0.00 & 21 & $0.032 /$ & 97.72 \\
\hline & & & & & & 4 & $0.032 /$ & -82.28 \\
\hline 15 & 17 & $0.992 /$ & -0.05 & $0.000 /$ & 0.00 & 12 & $0.005 /$ & -75.65 \\
\hline & & & & & & 22 & $0.000 /$ & 100.64 \\
\hline 16 & 18 & $0.881 /$ & -0.28 & $0.000 /$ & 0.00 & 13 & $0.070 /$ & -80.01 \\
\hline & & & & & & 23 & $0.006 /$ & 96.28 \\
\hline 17 & 19 & $0.992 /$ & -0.05 & $0.000 /$ & 0.00 & 6 & $0.016 /$ & -80.05 \\
\hline 18 & 20 & $0.000 /$ & 0.00 & $7.373 /$ & -85.20 & 15 & $5.739 /$ & 95.26 \\
\hline 19 & 21 & $0.878 /$ & -0.41 & $0.000 /$ & 0.00 & 16 & $0.032 /$ & -82.28 \\
\hline 20 & 22 & $0.992 /$ & -0.05 & $0.000 /$ & 0.00 & 17 & $0.000 /$ & -79.36 \\
\hline 21 & 23 & $0.881 /$ & -0.30 & $0.000 /$ & 0.00 & 18 & $0.006 /$ & -83.72 \\
\hline 22 & 24 & $0.854 /$ & -0.33 & $0.000 /$ & 0.00 & 4 & $0.191 /$ & -84.21 \\
\hline & & & & & & 31 & $0.139 /$ & 94.57 \\
\hline & & & & & & 32 & $0.052 /$ & 99.06 \\
\hline 23 & 25 & $0.990 /$ & -0.07 & $0.000 /$ & 0.00 & 28 & $0.004 /$ & 104.77 \\
\hline & & & & & & 9 & $0.004 /$ & -75.23 \\
\hline 24 & 26 & $0.990 /$ & -0.06 & $0.000 /$ & 0.00 & 29 & $0.004 /$ & 104.10 \\
\hline & & & & & & 3 & $0.004 /$ & -75.90 \\
\hline 25 & 27 & $0.854 /$ & -0.32 & $0.000 /$ & 0.00 & 30 & $0.058 /$ & 99.38 \\
\hline & & & & & & 4 & $0.128 /$ & -80.29 \\
\hline & & & & & & 10 & $0.070 /$ & 99.99 \\
\hline 26 & 28 & $0.991 /$ & -0.06 & $0.000 /$ & 0.00 & 25 & $0.004 /$ & -75.23 \\
\hline & & & & & & 33 & $0.001 /$ & 101.39 \\
\hline & & & & & & 41 & $0.000 /-$ & -121.54 \\
\hline 27 & 29 & $0.992 /$ & -0.06 & $0.000 /$ & 0.00 & 26 & $0.004 /$ & -75.90 \\
\hline & & & & & & 34 & $0.000 /$ & 104.61 \\
\hline & & & & & & 38 & $0.000 /-$ & -133.94 \\
\hline & & & & & & 38 & $0.000 /-$ & -133.94 \\
\hline 28 & 30 & $0.876 /$ & -0.30 & $0.000 /$ & 0.00 & 27 & $0.058 /$ & -80.62 \\
\hline & & & & & & 35 & $0.013 /$ & 97.10 \\
\hline 29 & 31 & $0.854 /$ & -0.33 & $0.000 /$ & 0.00 & 36 & $0.139 /$ & 94.57 \\
\hline & & & & & & 24 & $0.139 /$ & -85.43 \\
\hline 30 & 32 & $0.854 /$ & -0.33 & $0.000 /$ & 0.00 & 37 & $0.052 /$ & 99.06 \\
\hline & & & & & & 24 & $0.052 /$ & -80.94 \\
\hline 31 & 33 & $0.992 /$ & -0.06 & $0.000 /$ & 0.00 & 28 & $0.001 /$ & -78.61 \\
\hline 32 & 34 & $0.992 /$ & -0.06 & $0.000 /$ & 0.00 & 29 & $0.000 /$ & -75.39 \\
\hline 33 & 35 & $0.877 /$ & -0.33 & $0.000 /$ & 0.00 & 30 & $0.013 /$ & -82.90 \\
\hline 34 & 36 & $0.886 /$ & -0.36 & $0.000 /$ & 0.00 & 31 & $0.139 /$ & -85.43 \\
\hline 35 & 37 & $0.884 /$ & -0.36 & $0.000 /$ & 0.00 & 32 & $0.052 /$ & -80.94 \\
\hline 36 & 38 & $0.992 /$ & -0.06 & $0.000 /$ & 0.00 & 29 & $0.000 /$ & 46.06 \\
\hline & & & & & & 29 & $0.000 /$ & 46.06 \\
\hline 37 & 39 & $0.992 /$ & -0.06 & $0.000 /$ & 0.00 & 5 & $0.008 /$ & -80.49 \\
\hline 38 & 41 & $0.991 /$ & -0.06 & $0.000 /$ & 0.00 & 28 & $0.000 /$ & 58.46 \\
\hline 39 & 49 & $0.991 /$ & -0.06 & $0.000 /$ & 0.00 & 5 & $0.003 /$ & -77.60 \\
\hline 40 & 50 & $0.990 /$ & -0.07 & $0.000 /$ & 0.00 & 51 & $0.003 /$ & 103.35 \\
\hline
\end{tabular}




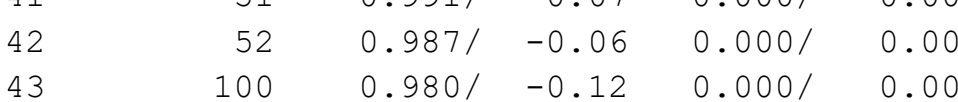

$30.140 /-81.22$

$30.140 /-81.22$

41

43

$0.000 /$

50

$0.003 /-76.65$

$4 \quad 2.500 /-85.10$

$10.182 / 99.42$

$2 \quad 2.233 /-84.17$

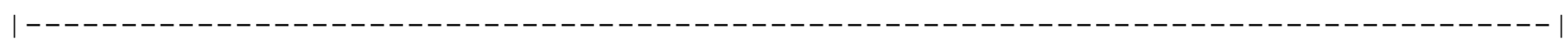




\begin{tabular}{|c|c|c|c|c|c|c|c|c|c|}
\hline \multirow{2}{*}{$\begin{array}{l}1 \\
2\end{array}$} & \multicolumn{2}{|c|}{$\%$} & name & \multicolumn{2}{|c|}{ baseMVA } & & & & \\
\hline & SYSTE & IEEE & Std_399-199 & 10 & & & & & \\
\hline 3 & $\div$ & & & & & & & & \\
\hline 4 & $\%$ & name & volts & & & & & & \\
\hline 5 & BUS & 1 & 1.00 & & & & & & \\
\hline 6 & BUS & 2 & 1.00 & & & & & & \\
\hline 7 & BUS & 3 & 1.00 & & & & & & \\
\hline 8 & BUS & 4 & 1.00 & & & & & & \\
\hline 9 & BUS & 5 & 1.00 & & & & & & \\
\hline 10 & BUS & 6 & 1.00 & & & & & & \\
\hline 11 & BUS & 8 & 1.00 & & & & & & \\
\hline 12 & BUS & 9 & 1.00 & & & & & & \\
\hline 13 & BUS & 10 & 1.00 & & & & & & \\
\hline 14 & BUS & 11 & 1.00 & & & & & & \\
\hline 15 & BUS & 12 & 1.00 & & & & & & \\
\hline 16 & BUS & 13 & 1.00 & & & & & & \\
\hline 17 & BUS & 15 & 1.00 & & & & & & \\
\hline 18 & BUS & 16 & 1.00 & & & & & & \\
\hline 19 & BUS & 17 & 1.00 & & & & & & \\
\hline 20 & BUS & 18 & 1.00 & & & & & & \\
\hline 21 & BUS & 19 & 1.00 & & & & & & \\
\hline 22 & BUS & 20 & 1.00 & & & & & & \\
\hline 23 & BUS & 21 & 1.00 & & & & & & \\
\hline 24 & BUS & 22 & 1.00 & & & & & & \\
\hline 25 & BUS & 23 & 1.00 & & & & & & \\
\hline 26 & BUS & 24 & 1.00 & & & & & & \\
\hline 27 & BUS & 25 & 1.00 & & & & & & \\
\hline 28 & BUS & 26 & 1.00 & & & & & & \\
\hline 29 & BUS & 27 & 1.00 & & & & & & \\
\hline 30 & BUS & 28 & 1.00 & & & & & & \\
\hline 31 & BUS & 29 & 1.00 & & & & & & \\
\hline 32 & BUS & 30 & 1.00 & & & & & & \\
\hline 33 & BUS & 31 & 1.00 & & & & & & \\
\hline 34 & BUS & 32 & 1.00 & & & & & & \\
\hline 35 & BUS & 33 & 1.00 & & & & & & \\
\hline 36 & BUS & 34 & 1.00 & & & & & & \\
\hline 37 & BUS & 35 & 1.00 & & & & & & \\
\hline 38 & BUS & 36 & 1.00 & & & & & & \\
\hline 39 & BUS & 37 & 1.00 & & & & & & \\
\hline 40 & BUS & 38 & 1.00 & & & & & & \\
\hline 41 & BUS & 39 & 1.00 & & & & & & \\
\hline 42 & BUS & 41 & 1.00 & & & & & & \\
\hline 43 & BUS & 49 & 1.00 & & & & & & \\
\hline 44 & BUS & 50 & 1.00 & & & & & & \\
\hline 45 & BUS & 51 & 1.00 & & & & & & \\
\hline 46 & BUS & 100 & 1.00 & & & & & & \\
\hline 47 & $\div$ & & & & & & & & \\
\hline 48 & $\frac{\circ}{0}$ & from & to & Rse & Xse & Gsh & Bsh & xo & Vis \\
\hline 49 & LINE & 1 & 3 & 0.00313 & 0.05324 & 0.00000 & 0.00000 & 0.00000 & 3 \\
\hline 50 & LINE & 2 & 4 & 0.00313 & 0.05324 & 0.00000 & 0.00000 & 0.00000 & 3 \\
\hline 51 & LINE & 5 & 39 & 0.04314 & 0.34514 & 0.00000 & 0.00000 & 0.00000 & 3 \\
\hline 52 & LINE & 5 & 49 & 0.05918 & 0.35510 & 0.00000 & 0.00000 & 0.00000 & 3 \\
\hline 53 & LINE & 6 & 11 & 0.05575 & 0.36240 & 0.00000 & 0.00000 & 0.00000 & 3 \\
\hline 54 & LINE & 6 & 19 & 0.01218 & 0.14616 & 0.00000 & 0.00000 & 0.00000 & 3 \\
\hline 55 & LINE & 12 & 17 & 0.06843 & 0.44477 & 0.00000 & 0.00000 & 0.00000 & 3 \\
\hline
\end{tabular}




\begin{tabular}{|c|c|c|c|c|c|c|c|c|}
\hline 56 & LINE & 13 & 18 & 0.05829 & 0.37888 & 0.00000 & 0.00000 & 0.00000 \\
\hline 57 & LINE & 15 & 20 & 0.01218 & 0.14616 & 0.00000 & 0.00000 & 0.00000 \\
\hline 58 & LINE & 16 & 21 & 0.15036 & 0.75178 & 0.00000 & 0.00000 & 0.00000 \\
\hline 59 & LINE & 25 & 28 & 0.05829 & 0.37888 & 0.00000 & 0.00000 & 0.00000 \\
\hline 60 & LINE & 26 & 29 & 0.05829 & 0.37888 & 0.00000 & 0.00000 & 0.00000 \\
\hline 61 & LINE & 27 & 30 & 0.05829 & 0.37888 & 0.00000 & 0.00000 & 0.00000 \\
\hline 62 & LINE & 31 & 36 & 0.02289 & 0.22886 & 0.00000 & 0.00000 & 0.00000 \\
\hline 63 & LINE & 32 & 37 & 0.10286 & 0.56573 & 0.00000 & 0.00000 & 0.00000 \\
\hline 64 & LINE & 50 & 51 & 0.06395 & 0.37796 & 0.00000 & 0.00000 & 0.00000 \\
\hline 65 & LINE & 3 & 5 & 0.00075 & 0.00063 & 0.00000 & 0.00000 & 0.00000 \\
\hline 66 & LINE & 3 & 6 & 0.00109 & 0.00091 & 0.00000 & 0.00000 & 0.00000 \\
\hline 67 & LINE & 3 & 9 & 0.00150 & 0.00125 & 0.00000 & 0.00000 & 0.00000 \\
\hline 68 & LINE & 3 & 26 & 0.00157 & 0.00131 & 0.00000 & 0.00000 & 0.00000 \\
\hline 69 & LINE & 4 & 8 & 0.00076 & 0.00092 & 0.00000 & 0.00000 & 0.00000 \\
\hline 70 & LINE & 4 & 15 & 0.00227 & 0.00189 & 0.00000 & 0.00000 & 0.00000 \\
\hline 71 & LINE & 4 & 24 & 0.00118 & 0.00098 & 0.00000 & 0.00000 & 0.00000 \\
\hline 72 & LINE & 4 & 16 & 0.00274 & 0.00229 & 0.00000 & 0.00000 & 0.00000 \\
\hline 73 & LINE & 4 & 27 & 0.00143 & 0.00119 & 0.00000 & 0.00000 & 0.00000 \\
\hline 74 & LINE & 9 & 12 & 0.00038 & 0.00032 & 0.00000 & 0.00000 & 0.00000 \\
\hline 75 & LINE & 9 & 25 & 0.00424 & 0.00353 & 0.00000 & 0.00000 & 0.00000 \\
\hline 76 & LINE & 10 & 13 & 0.00046 & 0.00039 & 0.00000 & 0.00000 & 0.00000 \\
\hline 77 & LINE & 10 & 27 & 0.00110 & 0.00091 & 0.00000 & 0.00000 & 0.00000 \\
\hline 78 & LINE & 17 & 22 & 0.03813 & 0.02451 & 0.00000 & 0.00000 & 0.00000 \\
\hline 79 & LINE & 18 & 23 & 0.03813 & 0.02451 & 0.00000 & 0.00000 & 0.00000 \\
\hline 80 & LINE & 24 & 31 & 0.00079 & 0.00065 & 0.00000 & 0.00000 & 0.00000 \\
\hline 81 & LINE & 24 & 32 & 0.00112 & 0.00093 & 0.00000 & 0.00000 & 0.00000 \\
\hline 82 & LINE & 28 & 33 & 0.03813 & 0.02451 & 0.00000 & 0.00000 & 0.00000 \\
\hline 83 & LINE & 28 & 41 & 0.03429 & 0.02105 & 0.00000 & 0.00000 & 0.00000 \\
\hline 84 & LINE & 29 & 34 & 0.03813 & 0.02451 & 0.00000 & 0.00000 & 0.00000 \\
\hline 85 & LINE & 29 & 38 & 0.08024 & 0.07732 & 0.00000 & 0.00000 & 0.00000 \\
\hline 86 & LINE & 29 & 38 & 0.08024 & 0.07732 & 0.00000 & 0.00000 & 0.00000 \\
\hline 87 & LINE & 30 & 35 & 0.03813 & 0.02451 & 0.00000 & 0.00000 & 0.00000 \\
\hline 88 & LINE & 50 & 3 & 0.00243 & 0.00485 & 0.00000 & 0.00000 & 0.00000 \\
\hline 89 & LINE & 50 & 3 & 0.00243 & 0.00485 & 0.00000 & 0.00000 & 0.00000 \\
\hline 90 & LINE & 100 & 1 & 0.00139 & 0.00296 & 0.00000 & 0.00000 & 0.00000 \\
\hline 91 & LINE & 100 & 2 & 0.00139 & 0.00296 & 0.00000 & 0.00000 & 0.00000 \\
\hline
\end{tabular}

$92 \div$

94 GENERATOR

95 GENERATOR 50

96 GENERATOR 100

$97 \%$

$98 \div$

99 MOTOR

100 MOTOR

101 MOTOR

102 MOTOR

103 MOTOR

104 MOTOR

105 MOTOR

106 MOTOR

107 MOTOR

108 MOTOR

109 MOTOR

110 MOTOR

bus
4
50
100

bus
11
17
17
18
18
19
19
20
20
21
22
23

0.003

0.002

$\mathrm{Xs}$
0.000

$\mathrm{xp}$

0.000

0.000

0.000

0.000

0.000

$\begin{array}{ll}R & X S \\ 0.352 & 0.000 \\ 0.338 & 0.000 \\ 0.802 & 0.000 \\ 0.338 & 0.000 \\ 0.802 & 0.000 \\ 0.057 & 0.000 \\ 0.047 & 0.000 \\ 0.067 & 0.000 \\ 0.060 & 0.000 \\ 0.320 & 0.000 \\ 1.398 & 0.000 \\ 1.398 & 0.000\end{array}$

Xpp
0.102

$\mathrm{x} 2$

xo

0.072

0.000

0.000

0.010

$0.000 \quad 0.000$

$0.000 \quad 0.000$

bus

17

17

18

18

19

19

20

20

21

23 $\mathrm{xp}$

0.000

Xpp

0.000

0.000

0.000

0.000

0.000

0.000

0.000

0.000

0.000

0.000

0.000

4.219

x2

0.000

$\mathrm{x} 0$

3.384

0.000

0.000

4.008

0.000

0.000

3.384

0.000

0.000

4.008

0.000

.000

1.484

0.000

0.000

0.703

0.000

0.000

1.005

0.000

.000

1.556

0.000

0.000

3.835

0.000

0.000

19.571

0.000

0.000

19.571

0.000

0.000 


\begin{tabular}{|c|c|c|c|c|c|c|c|c|}
\hline 111 MOTOR & & 28 & 0.697 & 0.000 & 0.000 & 6.972 & 0.000 & 0.000 \\
\hline 112 MOTOR & & 28 & 0.802 & 0.000 & 0.000 & 4.008 & 0.000 & 0.000 \\
\hline 13 MOTOR & & 29 & 0.321 & 0.000 & 0.000 & 3.206 & 0.000 & 0.000 \\
\hline 14 MOTOR & & 29 & 1.199 & 0.000 & 0.000 & 5.998 & 0.000 & 0.000 \\
\hline 15 MOTOR & & 30 & 0.431 & 0.000 & 0.000 & 5.166 & 0.000 & 0.000 \\
\hline $16 \mathrm{MOTOR}$ & & 30 & 1.116 & 0.000 & 0.000 & 5.578 & 0.000 & 0.000 \\
\hline 17 MOTOR & & 33 & 0.809 & 0.000 & 0.000 & 9.701 & 0.000 & 0.000 \\
\hline 18 MOTOR & & 34 & 3.621 & 0.000 & 0.000 & 25.354 & 0.000 & 0.000 \\
\hline 19 MOTOR & & 35 & 0.809 & 0.000 & 0.000 & 9.701 & 0.000 & 0.000 \\
\hline 20 MOTOR & & 36 & 0.025 & 0.000 & 0.000 & 0.818 & 0.000 & 0.000 \\
\hline 21 MOTOR & & 37 & 0.246 & 0.000 & 0.000 & 2.952 & 0.000 & 0.000 \\
\hline 22 MOTOR & & 37 & 1.859 & 0.000 & 0.000 & 9.296 & 0.000 & 0.000 \\
\hline 23 MOTOR & & 39 & 0.034 & 0.000 & 0.000 & 1.005 & 0.000 & 0.000 \\
\hline 24 MOTOR & & 49 & 0.264 & 0.000 & 0.000 & 2.640 & 0.000 & 0.000 \\
\hline 25 MOTOR & & 51 & 1.432 & 0.000 & 0.000 & 10.020 & 0.000 & 0.000 \\
\hline $126 \mathrm{MOTOR}$ & & 51 & 0.408 & 0.000 & 0.000 & 4.893 & 0.000 & 0.000 \\
\hline 27 MOTOR & & 8 & 0.006 & 0.000 & 0.000 & 0.222 & 0.000 & 0.000 \\
\hline $8 \%$ & & & & & & & & \\
\hline $9 \%$ & bus & Calc Typ & & lc_time & $(0-A)$ & 1-Subtr & noingt & 2-Transient;3-Steady-state) \\
\hline 30 FAULT & 20 & 3P & & 1 & & & & \\
\hline
\end{tabular}




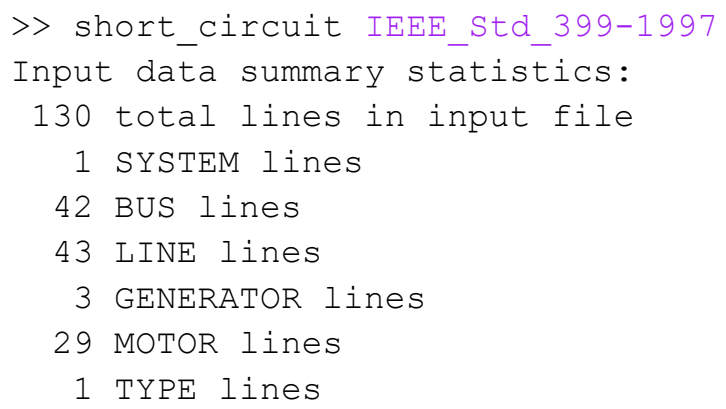

Results for System Name "IEEE_Std_399-1997"

Symmetrical Three-Phase Fault at Bus 20

Calculating Subtransient Currents

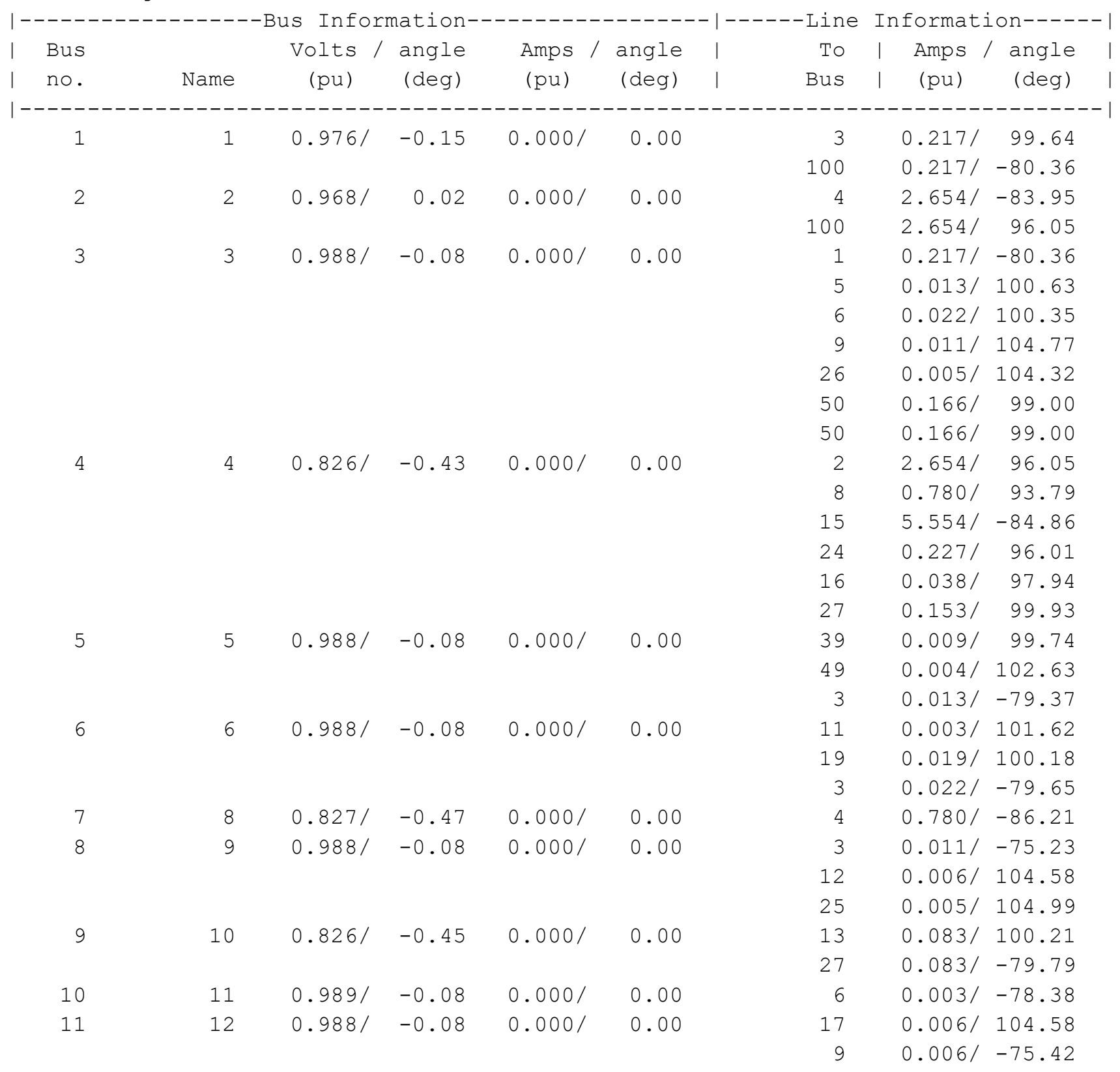




\begin{tabular}{|c|c|c|c|c|c|c|c|c|}
\hline \multirow{2}{*}{12} & \multirow[t]{2}{*}{13} & \multirow[t]{2}{*}{$0.827 /$} & \multirow[t]{2}{*}{-0.45} & \multirow[t]{2}{*}{$0.000 /$} & \multirow[t]{2}{*}{0.00} & 18 & $0.083 /$ & 100.21 \\
\hline & & & & & & 10 & $0.083 /$ & -79.79 \\
\hline \multirow[t]{2}{*}{13} & \multirow[t]{2}{*}{15} & \multirow[t]{2}{*}{$0.815 /$} & \multirow[t]{2}{*}{0.38} & \multirow[t]{2}{*}{$0.000 /$} & \multirow[t]{2}{*}{0.00} & 20 & $5.554 /$ & -84.86 \\
\hline & & & & & & 4 & $5.554 /$ & 95.14 \\
\hline \multirow[t]{2}{*}{14} & \multirow[t]{2}{*}{16} & \multirow[t]{2}{*}{$0.826 /$} & \multirow[t]{2}{*}{-0.44} & \multirow[t]{2}{*}{$0.000 /$} & \multirow[t]{2}{*}{0.00} & 21 & $0.038 /$ & 97.94 \\
\hline & & & & & & 4 & $0.038 /$ & -82.06 \\
\hline \multirow[t]{2}{*}{15} & \multirow[t]{2}{*}{17} & $0.990 /$ & -0.06 & $0.000 /$ & 0.00 & 12 & $0.006 /$ & -75.42 \\
\hline & & & & & & 22 & $0.000 /$ & 100.87 \\
\hline 16 & 18 & $0.858 /$ & -0.38 & $0.000 /$ & 0.00 & 13 & $0.083 /$ & -79.79 \\
\hline & & & & & & 23 & $0.007 /$ & 96.50 \\
\hline 17 & 19 & $0.991 /$ & -0.06 & $0.000 /$ & 0.00 & 6 & $0.019 /$ & -79.82 \\
\hline 18 & 20 & $0.000 /$ & 7.13 & $7.188 /$ & -85.30 & 15 & $5.554 /$ & 95.14 \\
\hline 19 & 21 & $0.855 /$ & -0.54 & $0.000 /$ & 0.00 & 16 & $0.038 /$ & -82.06 \\
\hline 20 & 22 & $0.990 /$ & -0.07 & $0.000 /$ & 0.00 & 17 & $0.000 /$ & -79.13 \\
\hline 21 & 23 & $0.859 /$ & -0.40 & $0.000 /$ & 0.00 & 18 & $0.007 /$ & -83.50 \\
\hline 22 & 24 & $0.826 /$ & -0.45 & $0.000 /$ & 0.00 & 4 & $0.227 /$ & -83.99 \\
\hline & & & & & & 31 & $0.166 /$ & 94.80 \\
\hline & & & & & & 32 & $0.061 /$ & 99.28 \\
\hline 23 & 25 & $0.988 /$ & -0.08 & $0.000 /$ & 0.00 & 28 & $0.005 /$ & 104.99 \\
\hline & & & & & & 9 & $0.005 /$ & -75.01 \\
\hline 24 & 26 & $0.988 /$ & -0.08 & $0.000 /$ & 0.00 & 29 & $0.005 /$ & 104.32 \\
\hline & & & & & & 3 & $0.005 /$ & -75.68 \\
\hline 25 & 27 & $0.826 /$ & -0.44 & $0.000 /$ & 0.00 & 30 & $0.069 /$ & 99.60 \\
\hline & & & & & & 4 & $0.153 /$ & -80.07 \\
\hline & & & & & & 10 & $0.083 /$ & 100.21 \\
\hline 26 & 28 & $0.990 /$ & -0.07 & $0.000 /$ & 0.00 & 25 & $0.005 /$ & -75.01 \\
\hline & & & & & & 33 & $0.001 /$ & 101.62 \\
\hline & & & & & & 41 & $0.000 /$ & -121.54 \\
\hline 27 & 29 & $0.990 /$ & -0.07 & $0.000 /$ & 0.00 & 26 & $0.005 /$ & -75.68 \\
\hline & & & & & & 34 & $0.000 /$ & 104.84 \\
\hline & & & & & & 38 & $0.000 /-$ & $-133 \cdot 94$ \\
\hline & & & & & & 38 & $0.000 /$ & -133.94 \\
\hline 28 & 30 & $0.853 /$ & -0.40 & $0.000 /$ & 0.00 & 27 & $0.069 /$ & -80.40 \\
\hline & & & & & & 35 & $0.015 /$ & 97.32 \\
\hline 29 & 31 & $0.827 /$ & -0.46 & $0.000 /$ & 0.00 & 36 & $0.166 /$ & 94.80 \\
\hline & & & & & & 24 & $0.166 /$ & -85.20 \\
\hline 30 & 32 & $0.827 /$ & -0.45 & $0.000 /$ & 0.00 & 37 & $0.061 /$ & 99.28 \\
\hline & & & & & & 24 & $0.061 /$ & -80.72 \\
\hline 31 & 33 & $0.990 /$ & -0.07 & $0.000 /$ & 0.00 & 28 & $0.001 /$ & -78.38 \\
\hline 32 & 34 & $0.990 /$ & -0.07 & $0.000 /$ & 0.00 & 29 & $0.000 /$ & -75.16 \\
\hline 33 & 35 & $0.853 /$ & -0.44 & $0.000 /$ & 0.00 & 30 & $0.015 /$ & -82.68 \\
\hline 34 & 36 & $0.865 /$ & -0.48 & $0.000 /$ & 0.00 & 31 & $0.166 /$ & -85.20 \\
\hline 35 & 37 & $0.862 /$ & -0.48 & $0.000 /$ & 0.00 & 32 & $0.061 /$ & -80.72 \\
\hline 36 & 38 & $0.990 /$ & -0.07 & $0.000 /$ & 0.00 & 29 & $0.000 /$ & 46.06 \\
\hline & & & & & & 29 & $0.000 /$ & 46.06 \\
\hline 37 & 39 & $0.991 /$ & -0.07 & $0.000 /$ & 0.00 & 5 & $0.009 /$ & -80.26 \\
\hline 38 & 41 & $0.990 /$ & -0.07 & $0.000 /$ & 0.00 & 28 & $0.000 /$ & 58.46 \\
\hline 39 & 49 & $0.989 /$ & -0.07 & $0.000 /$ & 0.00 & 5 & $0.004 /$ & -77.37 \\
\hline & 50 & $0.988 /$ & -0.09 & $0.000 /$ & 0.00 & 51 & $0.003 /$ & 103.57 \\
\hline & & & & & & 3 & $0.166 /$ & -81.00 \\
\hline
\end{tabular}




$\begin{array}{rrrrrr}41 & 51 & 0.990 / & -0.08 & 0.000 / & 0.00 \\ 42 & 100 & 0.976 / & -0.14 & 0.000 / & 0.00\end{array}$

$3 \quad 0.166 /-81.00$

42

$50 \quad 0.003 /-76.43$

$10.217 / 99.64$

$2 \quad 2.654 /-83.95$

$>>$ 
1 function short_circuit(filename)

2 \% MATLAB fault current analysis program to perform fault current

3 o calculations for three-phase, single line-to-ground, line-to-line,

$4 \%$ or double line-to-ground faults in the subtransient, transient, or

5 \% steady-state periods. The input to this program is a data set of input

6 응 linescribing a power system read from an input file. The only

7 argument is the name of the input file. The output from this program

$8 \%$ calculates the voltages and fault currents in each phase at each bus and

$9 \%$ in each transmission line in the power system for the particular type of

$10 \%$ fault being analyzed.

$11 \div$

12 \% The input file may contain up to six different types of input lines

13 \% used to describe the power system: "SYSTEM", "BUS", "LINE", "GENERATOR",

14 으임, and "FAULT" lines, respectively.

$15 \%$

16 \% The "SYSTEM" line specifies the characteristics of the power system

$17 \div$ as shown below.

$18 \div$

$19 \div$ SYSTEM name baseMVA

$20 \div$

$21 \div$ where

22 을 $\quad$ The name of the power system

$23 \%$ baseMVA = The base apparent power of the power system (in MVA)

$24 \div$

25 \% The "BUS" line specifies the characteristics of a bus in the

26 power system as shown below.

$27 \%$

$28 \div$ BUS name volts

$29 \div$

30 \% where

31 을 $=$ The name of the bus

$32 \%$ volts = The pre-fault voltage at the bus

$33 \div$

34 \% The "LINE" line specifies the characteristics of a transmission

$35 \%$ line in the power system as shown below. Note that transformers

36 ot the sending and receiving ends of transmission lines are treated

37 응 additional "transmission lines".

$38 \%$

39 늘 from to Rse Xse Gsh Bsh XO Vis

$40 \div$

$41 \%$ where

$42 \%$ from = The name of the "from" bus

$43 \%$ to = The name of the "to" bus

$44 \%$ Rse = Per-unit series resistance

$45 \div \mathrm{Xse}=$ Per-unit series reactance

$46 \%$ Gsh $=$ Per-unit shunt conductance

$47 \%$ Bsh = Per-unit shunt susceptance

$48 \% \mathrm{x} 0=$ Per-unit series zero-sequence reactance

$49 \div$ Vis = Zero-sequence visibility flag:

$50 \div \quad 0$ - Visible to neither bus

51 음 1 - Visible to the "from" bus only

52 \% 2 - Visible to the "to" bus only

$53 \div \quad 3$ - Visible to both buses

$54 \%$

55 \% The "GENERATOR" line specifies the characteristics of a generator 
56 in the power system as shown below.

$57 \div$

$58 \div$ GENERATOR bus R Xs Xp Xpp X2 X0

$59 \div$

$60 \%$ where

$61 \%$ bus = The name of the bus where the generator is connected

$62 \% \mathrm{R}=$ Per-unit resistance

$63 \div \mathrm{XS} \quad=$ Per-unit synchronous reactance

$64 \% \mathrm{xp} \quad=$ Per-unit transient reactance

$65 \% \mathrm{xpp} \quad=$ Per-unit subtransient reactance

$66 \% \mathrm{x} 2=$ Per-unit negative-sequence reactance

$67 \%$ X0 $\quad$ Per-unit zero-sequence reactance

$68 \div$

69 \% The "MOTOR" line specifies the characteristics of a synchronous motor

70 in the power system as shown below.

$71 \%$

$72 \div$ MOTOR bus R Xs Xp Xpp X2 X0

$73 \div$

$74 \div$ where

$75 \%$ bus = The name of bus where the motor is connected

$76 \% \mathrm{R} \quad=$ Per-unit resistance

$77 \div \quad \mathrm{Xs} \quad=$ Per-unit synchronous reactance

$78 \div \quad \mathrm{xp} \quad=$ Per-unit transient reactance

$79 \div \mathrm{xpp} \quad=$ Per-unit subtransient reactance

$80 \div \quad \mathrm{x} 2=$ Per-unit negative-sequence reactance

$81 \% \mathrm{x} 0$ = Per-unit zero-sequence reactance

$82 \div$

$83 \%$ Finally, the "FAULT" line specifies the location, type, and time period

$84 \%$ (subtransient, etc.) of the fault as shown below.

$85 \%$

$86 \div$ FAULT bus calc_type calc_time

$87 \%$

$88 \div$ where

$89 \%$ bus = The name of the bus where the fault occurs

$90 \%$ calc_type = The type of fault that occurs at that bus:

$91 \div 3 P$ - symmetrical three-phase fault

$92 \div \quad$ LG - Single line-to-ground fault

$93 \div$

$94 \div$

$95 \div$

101 \% These six different types of input lines describing the power system

102 \% should appear in the input file in the following order:

$103 \%$

$104 \div$

$105 \%$ BUS

$106 \div$ LINE ...

$107 \div$ GENERATOR ...

$108 \div$ MOTOR ...

$109 \div$ FAULT ... 
111 o Read the input lines describing the power system from the input file.

112 [bus, line, system, gen, mot, fault] = read_file(filename);

113

114 으의 fault analysis on the power system.

115 if fault.calc_time > 0

116

117

118

\% Create the positive-, negative-, and zero-sequence bus admittance

\% matrices for the power system.

119

[Ybus1, Ybus2, Ybus0] = build_Ybus(bus, line, gen, mot, fault);

120

121

122

o Create the positive-sequence bus impedance matrix for the power

\% system.

123

Zbus1 = inv(Ybus1);

124

125

126

127

128

129

130

131

132

133

134

135

136

137

138

139

140

\% Create the negative-sequence bus impedance matrix for the power

\% system.

if $\sim$ strcmpi (fault.type, '3P')

Zbus2 = inv(Ybus2);

else

end

o Create the zero-sequence bus impedance matrix for the power system.

if strcmpi(fault.type, 'SLG') || strcmpi(fault.type, 'DLG')

Zbus0 = inv(Ybus0);

else

Zbus0 = zeros $($ size $($ Ybus0));

end

\% Solve for the voltages and fault currents in each phase at each bus

\% in the power system for the particular type of fault being analyzed.

bus = solve (bus, Zbus1, Zbus2, Zbus0, fault);

142

143

144

\% Display the voltages and fault currents in each phase at each bus and

145

146

147

148

149

150

151

152 else

153

o in each transmission line in the power system for the particular type

o of fault being analyzed.

if strcmpi (fault.type, '3P')

report(system, bus, line, Ybusl, fault);

else

report_2(system, bus, line, Ybus1, Ybus2, Ybus0, fault); end

154

155

156

157

158

159

160

161

162

163

164

for $i=1: 3$

\% Set the time period for the fault analysis.

fault.calc_time $=i$;

o Create the positive-, negative-, and zero-sequence bus admittance

\% matrices for the power system.

[Ybus1, Ybus2, Ybus0] = build_Ybus(bus, line, gen, mot, fault);

\% Create the positive-sequence bus impedance matrix for the power

\% system.

165

Zbus1 = inv(Ybus1); 
- Create the negative-sequence bus impedance matrix for the power

$201 \%$ Function read_file reads a data set describing a power system from an

202 \% input file.

203

$204 \%$ Validate the number of input arguments in the call to the currently

$205 \%$ executing function.

206 narginchk $(1,1)$;

207

$208 \%$ Initialize counters to count the number of occurences of each of the

209 \% different types of lines encountered while reading from the input file.

210 numsystem $=0 ; \quad \quad$ O Number of occurences of SYSTEM lines

211 numBus $\quad=0$;

212 numLine $\quad=0$;

213 numGen $\quad=0$;

214 numMot $=0$;

215 numFault $=0$;

216 numComment $=0$;

217 numInvalid $=0$;

218 i_line $\quad=0$;

$219 \operatorname{mot} \quad=[]$;

220

\% Number of occurences of BUS lines

\% Number of occurences of LINE lines

\% Number of occurences of GENERATOR lines

\% Number of occurences of MOTOR lines

\% Number of occurences of FAULT lines

- Number of occurences of comment lines

- Number of occurences of invalid lines

\% Current line number

o Initialize motor array 
221 open the input file for reading. If fopen cannot open the input file,

222 늘 fileID $=-1$.

223 [fileID] = fopen(filename, 'r');

224

225 \% Check for an error opening the input file. If there is an error opening

226 o the input file, throw an error and display an error message. Otherwise,

227 \% read the input file one line at a time to the end of the file.

228 if fileid $==-1$

229 errmsg = ['ERROR: MATLAB cannot open the input file: 'filename];

230 error(errmsg);

231 else

while feof(fileID) $==0$

tline = fgetl(fileID);

\% Test for end of input file

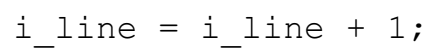

\% Read line from input file

234

235

236

237

238

239

240

241

242

243

244

245

246

247

248

249

250

251

252

253

254

255

256

257

258

259

260

261

262

263

264

265

266

267

268

269

270

271

272

273

274

275

\% Determine the type of line read from the input file by comparing

o the first $n$ characters of the line with the each of the different

oline identifiers.

if strncmpi(tline, 'SYSTEM', 6) == 1

\% The line read from the input file is a SYSTEM line. Update

o the number of occurences of SYSTEM lines in the input file.

numsystem $=$ numbystem +1 ;

o Obtain the name of the power system.

index = strfind (tline, ' ');

for $i=1:($ length $($ index $)-1)$

if $(i n d e x(i+1)-i n d e x(i))>1$

system.name $=\operatorname{tline}((\operatorname{index}(i)+1):(i n d e x(i+1)-1))$; break;

end

end

\% Obtain the base apparent power of the power system (in MVA) . temp = sscanf (tline (index $(i+1)$ : length (tline)), '⿳9' ');

system.baseMVA $=\operatorname{temp}(1) ;$

elseif strncmpi(tline,'BUS', 3) == 1

\% The line read from the input file is a BUS line. Update the

\% number of occurences of BUS lines in the input file.

numBus $=$ numBus +1 ;

\% Confirm that the BUS line read from the input file has been

\% preceeded by the occurence of a SYSTEM line within the same

응 input file.

if numbystem $==0$

error(['ERROR: The input file must be formatted ' ...

'such that the occurence of any BUS lines is ' ...

'preceeded by the occurence of a SYSTEM line.']);

end

o Obtain the name of the bus.

index = strfind(tline, ' '); 


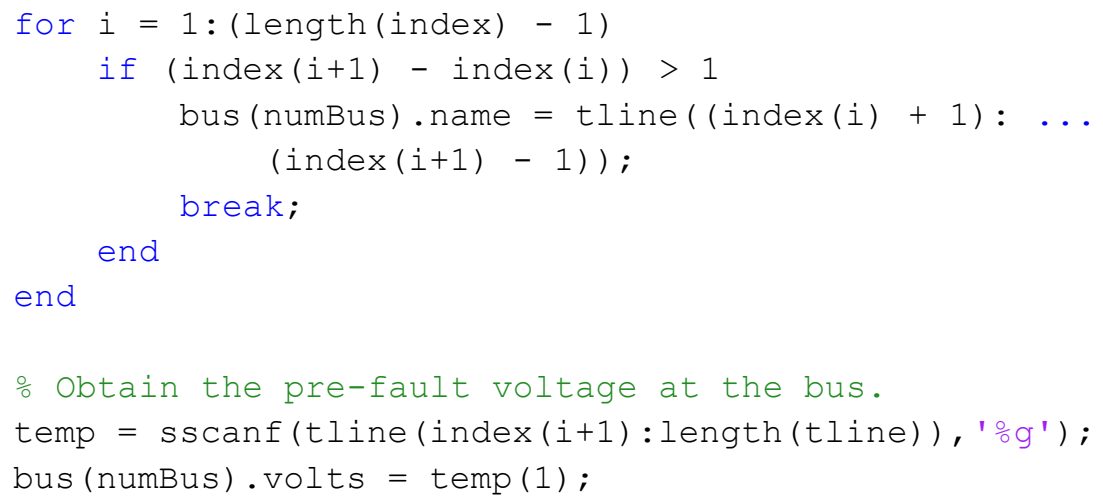




$$
\begin{array}{ll}
\text { line (numLine). Rse } & =\operatorname{temp}(1) ; \\
\text { line (numLine). Xse } & =\operatorname{temp}(2) ; \\
\text { line (numLine). Gsh } & =\operatorname{temp}(3) ; \\
\text { line (numLine). Bsh } & =\operatorname{temp}(4) ; \\
\text { line (numLine).X0 } & =\operatorname{temp}(5) ; \\
\text { line (numLine). vis } & =\operatorname{temp}(6) ;
\end{array}
$$


o Obtain the characteristics of the synchronous motor,

o including the per-unit resistance, per-unit synchronous

\% reactance, per-unit transient reactance, per-unit

$\%$ subtransient reactance, per-unit negative-sequence

o reactance, and per-unit zero-sequence reactance. temp $=\operatorname{sscanf}\left(\right.$ tline $($ index $(i+1):$ length $($ tline $\left.)),{ }^{\prime} \circ g^{\prime}\right)$; $\operatorname{mot}($ numMot) $\cdot \mathrm{R} \quad=\operatorname{temp}(1)$; $\operatorname{mot}($ numMot) $) \cdot \mathrm{Xs} \quad=\operatorname{temp}(2)$; $\operatorname{mot}($ numMot) $) \cdot \mathrm{xp} \quad=\operatorname{temp}(3)$; $\operatorname{mot}($ numMot) $\cdot \operatorname{xpp} \quad=\operatorname{temp}(4)$; $\operatorname{mot}($ numMot) $\cdot \mathrm{x} 2 \quad=\operatorname{temp}(5)$; $\operatorname{mot}($ numMot) $\cdot \mathrm{xO} \quad=\operatorname{temp}(6)$;

elseif strncmpi(tline, 'FAULT',5) == 1

\% The line read from the input file is a FAULT line. Update \% the number of occurences of FAULT lines in the input file. numFault $=$ numFault $+1 ;$

o Obtain the name of the bus where the fault develops. index = strfind(tline, ' '); 
\% the number of occurences of comment lines in the input file. numComment $=$ numComment +1 ;

else

\% The line read from the input file is an invalid line. Update \% the number of occurences of invalid lines in the input file. numInvalid $=$ numInvalid $+1 ;$

o Display a warning to alert the user of the invalid line in

\% the input file. disp(['WARNING: Invalid line ' int2str(i_line) ': ' tline]); end

end

- Process the input data to prepare for subsequent calculations and

o perform error checking on the input data to ensure validity.

- Initialize counter to count the total number of errors detected in

\% the input file.

numError $=0$;

\% Confirm that there is one and only one occurence of a SYSTEM line in o the input file.

if numbystem $==0$

numError $=$ numError +1 ;

disp(['ERROR: No SYSTEM line in the input file. The input ' ... 'file should contain one and only one SYSTEM line.']);

end

if numbystem $>1$

numError $=$ numError +1 ;

disp (['ERROR: More than one SYSTEM line in the input file. ' ... 'The input file should contain one and only one SYSTEM ' ...

'line.']);

end

- Initialize counter to count the number of transmission lines

o terminated on each bus in the power system.

for $i=1$ :numBus

bus(i). numLine $=0$;

end

- Count the number of transmission lines terminated on each bus in the

o power system and confirm that each LINE in the power system has a

o valid "from" and "to" bus name associated with it that corresponds

o to the name of an indentified bus in the power system.

for $i=1$ :numLine

line (i).from no $=0$;

line(i).to no $=0$;

for $j=1$ :numBus

if strompi(line(i).from_name, bus(j).name)

bus $(j)$.numLine $=$ bus $(j)$.numLine +1 ;

line (i).from_no $=j$;

end 


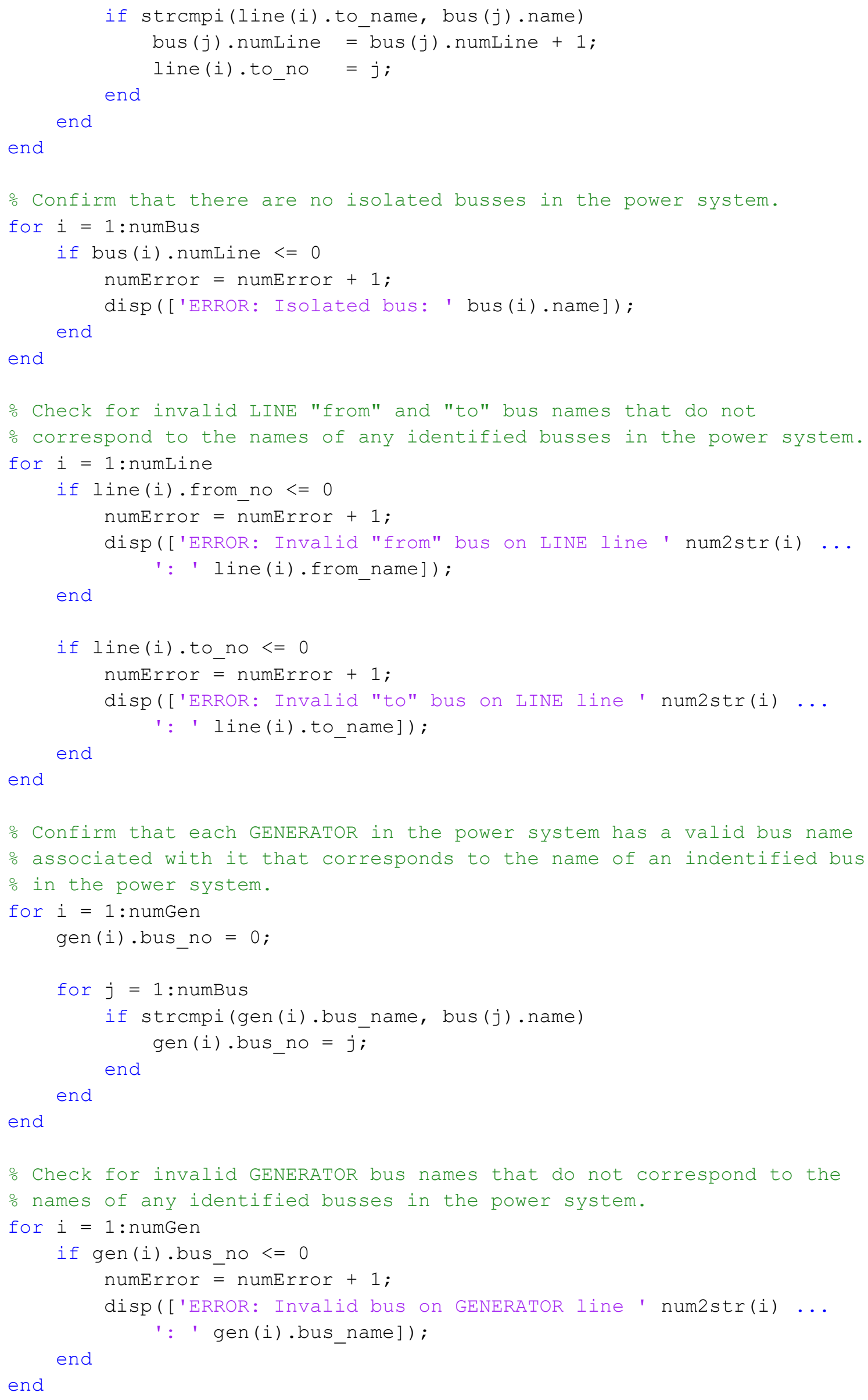


\% Confirm that each MOTOR in the power system has a valid bus name

\% Check for invalid MOTOR bus names that do not correspond to the names ㅇ of any identified busses in the power system. 


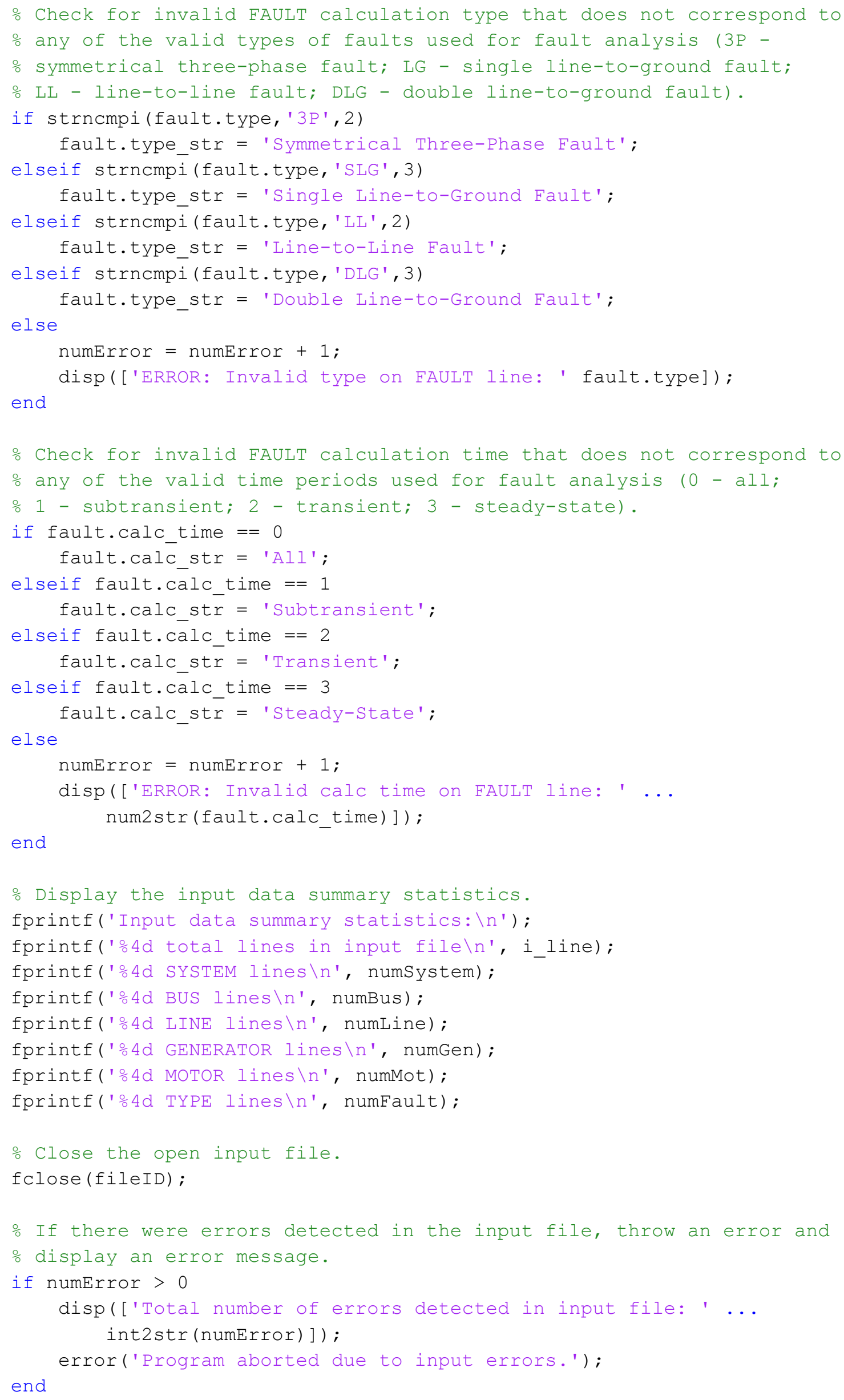


665 function [Ybus1, Ybus2, Ybus0] = build_Ybus (bus, line, gen, mot, fault)

$666 \%$ Function build_Ybus calculates the bus admittance matrix of the power

$667 \%$ system according to the following rules:

$668 \%$

$669 \div 1)$ The diagonal elements Ybus $(k, k)$ of the bus admittance matrix are $670 \%$ equal to the sum of all admittances directly connected to bus $\mathrm{k}$. The

$671 \%$ diagonal elements are referred to as the self-admittances of the

$672 \div$ busses.

673 \% 2) The off-diagonal elements Ybus $(k, n)$ of the bus admittance matrix are

$674 \%$ equal to the negative of the sum of all admittances directly

675 connected between busses $k$ and $n$. The off-diagonal elements are

$676 \%$ referred to as the mutual admittances of the busses.

$677 \div$

678 \% The bus admittance matrix includes the admittances of all transmission

679 \% lines, transformers, etc., connected between busses and also includes

$680 \%$ the admittances of the loads or generators themselves connected at each

$681 \div$ bus.

682

$683 \%$ Validate the number of input arguments in the call to the currently

$684 \div$ executing function.

685 narginchk $(5,5)$;

686

687 Obtain the number of buses in the power system.

688 numBus = length (bus);

689

$690 \%$ Initialize the positive-, negative-, and zero-sequence bus admittance

691 \% matrices for the power system.

692 Ybus1 = zeros (numBus, numBus);

693 Ybus2 = zeros (numBus, numBus);

694 Ybus0 = zeros (numBus, numBus);

695

696 oreate the positive-sequence bus admittance matrix for the power system.

697 for $i=1:$ length (line)

698

699

700

701

702

703

704

705

706

707

708

709

710

711

712

713

714

715 obtain the positive-sequence bus admittance matrix indices.

$\mathrm{k}=$ line(i).from_no;

$\mathrm{n}=$ line(i).to_no;

obtain the series positive-sequence impedance of the transmission

\% line from the series resistance and series positive-sequence

\% reactance. Convert the series positive-sequence impedance of the

otransmission line to a series positive-sequence admittance and

\% obtain the shunt admittance of the transmission line from the shunt

\% conductance and shunt susceptance.

Zse $=$ line (i).Rse $+1 j *$ line (i).Xse;

Yse $=1.0 /$ Zse;

Ysh $=$ line $(i) \cdot$ Gsh $+1 j * l i n e(i) \cdot$ Bsh;

\% Calculate the diagonal elements of the positive-sequence bus

\% admittance matrix corresponding to the positive-sequence self-

\% admittances of the busses. 
\% Calculate the off-diagonal elements of the positive-sequence bus

766 - Add the positive-sequence admittances of the motors connected at each bus 767 \% to the positive-sequence bus admittance matrix.

768 for $i=1:$ length (mot) 
$\operatorname{Ybus} 2(k, k)=\operatorname{Ybus} 2(k, k)+Y s e ;$

$\operatorname{Ybus} 2(\mathrm{n}, \mathrm{n})=\operatorname{Ybus} 2(\mathrm{n}, \mathrm{n})+$ Yse;

\% Calculate the off-diagonal elements of the negative-sequence bus \% admittance matrix corresponding to the negative-sequence mutual

o admittances of the busses.

Ybus2 $(k, n)=\operatorname{Ybus} 2(k, n)-Y s e$;

Ybus2 $(n, k)=\operatorname{Ybus} 2(k, n)$;

\% The transmission line is represented by the pi model in which the ototal shunt admittance distributed over the entire length of the o transmission line is divided into two halves and placed at the \% sending and receiving ends of the transmission line. Add half of o the total shunt admittance of the transmission line to the busses \% at each end of the transmission line.

Ybus2 $(k, k)=\operatorname{Ybus} 2(k, k)+Y s h / 2$;

Ybus2 $(n, n)=\operatorname{Ybus} 2(n, n)+Y s h / 2$;

end

\% Add the negative-sequence admittances of the generators connected at \% each bus to the negative-sequence bus admittance matrix. for $i=1$ : length (gen)

\% Obtain the negative-sequence bus admittance matrix index. $\mathrm{k}=$ gen(i).bus_no;

\% Obtain the series negative-sequence impedance of the generator

\% from the series resistance and series negative-sequence

\% reactance. Convert the series negative-sequence impedance of the \% generator to a series negative-sequence admittance.

$Z 2=\operatorname{gen}(i) \cdot R+1 j * g e n(i) \cdot X 2 ;$

$\mathrm{Y} 2=1.0 /(\mathrm{Z} 2)$;

\% Calculate the diagonal elements of the negative-sequence bus

\% admittance matrix corresponding to the negative-sequence self-

\% admittances of the busses.

$\operatorname{Ybus} 2(k, k)=\operatorname{Ybus} 2(k, k)+Y 2$;

end

- Add the negative-sequence admittances of the motors connected at each

\% bus to the negative-sequence bus admittance matrix.

for $i=1$ : length (mot)

\% Obtain the negative-sequence bus admittance matrix index.

$k=$ mot (i).bus_no;

\% Obtain the series negative-sequence impedance of the motor from

\% the series resistance and series negative-sequence reactance.

\% Convert the series negative-sequence impedance of the motor to a

\% series negative-sequence admittance.

$\mathrm{z} 2=\operatorname{mot}(i) \cdot \mathrm{R}+1 j * \operatorname{mot}(i) \cdot \mathrm{X} 2$;

$\mathrm{Y} 2=1.0 /(\mathrm{Z} 2)$;

\% Calculate the diagonal elements of the negative-sequence bus o admittance matrix corresponding to the negative-sequence self- 

end

884 end

885

886 \% Create the zero-sequence bus admittance matrix for the power system if

887 \% it becomes unbalanced during an unsymmetrical ground fault (applies to

$888 \%$ single line-to-ground faults and double line-to-ground faults denoted

$889 \%$ by 'SLG' and 'DLG', respectively).

890 if strcmpi (fault.type,'SLG') || strcmpi (fault.type, 'DLG')

for $i=1:$ length (line)

\% Obtain the series zero-sequence impedance of the transmission

\% line from the series resistance and series zero-sequence

o reactance. Convert the series zero-sequence impedance of the

\% transmission line to a series zero-sequence admittance and

o obtain the shunt admittance of the transmission line from the

\% shunt conductance and shunt susceptance.

$Z 0=$ line $(i) \cdot$ Rse $+1 j *$ line $(i) \cdot X 0 ;$

YO $=1.0 / \mathrm{ZO}$;

Ysh $=$ line(i).Gsh $+1 j * l i n e(i) \cdot$ Bsh;

- Calculate the diagonal elements of the zero-sequence bus

\% admittance matrix corresponding to the zero-sequence self-

\% admittances of the busses. If the zero-sequence visibility of

o the transmission line is equal to 1 or 3 , then the series zero-

o sequence admittance of the transmission line is visible to the

\% "from" bus.

if line(i). vis $==1$ || line(i). vis $==3$

$\operatorname{Ybus} 0(k, k)=\operatorname{Ybus} 0(k, k)+Y 0$;

end

916

917

918

919

920

921

922

923

924

925

926

927

928

929

930

931

932

933

934

935

의 the zero-sequence visibility of the transmission line is equal

\% to 2 or 3 , then the series zero-sequence admittance of the

o transmission line is visible to the "to" bus.

if line(i). vis $==2$ || line(i). vis $==3$

$\operatorname{Ybus} 0(n, n)=\operatorname{Ybus} 0(n, n)+Y 0$;

end

\% Calculate the off-diagonal elements of the zero-sequence bus

\% admittance matrix corresponding to the zero-sequence mutual

o admittances of the busses. If the zero-sequence visibility of the

otransmission line is equal to 3 , then the series zero-sequence

o admittance of the transmission line is visible to both busses.

if line(i). vis $==3$

$\operatorname{Ybus} 0(k, n)=\operatorname{Ybus} 0(k, n)-Y 0$;

$\operatorname{Ybus} 0(\mathrm{n}, \mathrm{k})=\operatorname{Ybus} 0(\mathrm{k}, \mathrm{n})$;

end

\% The transmission line is represented by the pi model in which the o total shunt admittance distributed over the entire length of the 


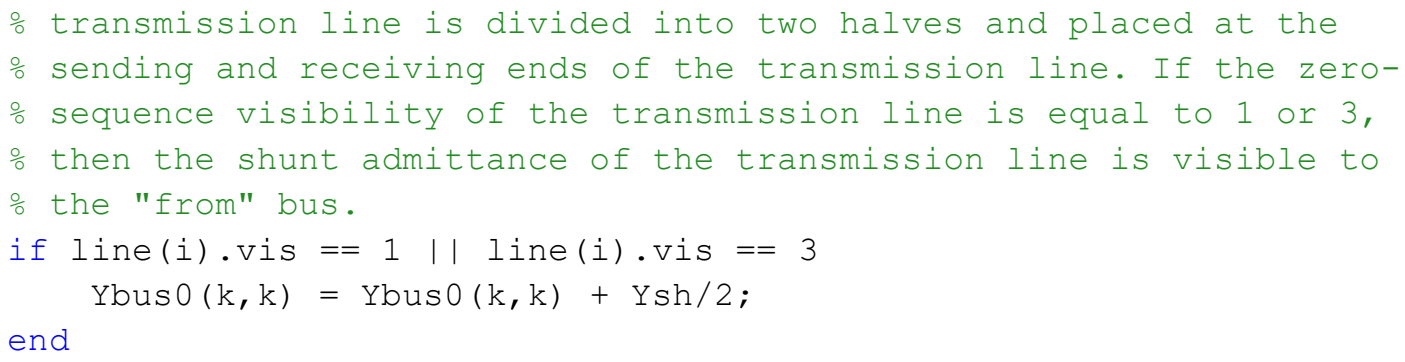


991 end

992 end

993

994

995

996 function bus = solve(bus, Zbus1, Zbus2, Zbus0, fault)

997 Function solve calculates the voltages and fault currents in each phase

998 \% at each bus in the power system for symmetrical three-phase, single

999 \% line-to-ground, line-to-line, or double line-to-ground faults in the

1000 \% subtransient, transient, or steady-state periods.

1001

1002 Validate the number of input arguments in the call to the currently

1003 \% executing function.

1004 narginchk $(5,5)$;

1005

1006 \% Define the 'a' constant to represent the operation of shifting a phasor

$1007 \%$ by an angle of 120 degrees. This operation is equivalent to multiplying

1008 \% the phasor by the 'a' constant defined below and each multiplication by

1009 \% the 'a' constant rotates the phasor by an angle of 120 degrees without

1010 \% changing its magnitude.

$1011 \mathrm{a}=-0.5+1 j * 0.86602540378444 ;$ o Rotate phasor by 120 degrees

1012 a2 $=-0.5-1 j * 0.86602540378444 ;$ Rotate phasor by 240 degrees

1013

1014 \% Determine the type of fault that develops on the power system and

1015 \% calculate the fault current in each phase at the faulted bus for the

$1016 \%$ particular type of fault being analyzed and the voltages in each phase

1017 \% at each bus during the fault (3P - symmetrical three-phase fault; LG -

1018 \% single line-to-ground fault; LL - line-to-line fault; DLG - double line-

$1019 \%$ to-ground fault).

1020 if strncmpi(fault.type, '3P',2)

1021 for $i=1$ : length (bus)

1022

1023

1024

1025

1026

1027

1028

1029

1030

1031

1032

1033

1034

1035

1036

1037

1038

1039

1040

1041

1042

1043

1044

1045
\% Calculate the fault current in each phase at the faulted bus for o a symmetrical three-phase fault.

if fault.bus_no $==i$

\% Calculate the positive-, negative-, and zero-sequence

o currents in phase a at the faulted bus.

bus(i).ial = bus(i). volts / Zbusl(i,i);

bus(i).ia2 $=0$;

bus(i).ia0 $=0$;

\% Calculate the fault current in each phase at the faulted bus

\% (i(1) - fault current in phase a; i(2) - fault current in

\% phase b; i(3) - fault current in phase c).

bus(i).i(1) = bus(i).ia1+bus(i).ia2+ bus(i).ia0;

bus(i).i(2) = a $2 *$ bus(i).ial $+a * b u s(i) \cdot i a 2+$ bus(i).ia0;

bus(i).i(3) $=a *$ bus(i).ial $+a 2 *$ bus(i).ia2 + bus(i).ia0;

else

bus (i).ial $=0$;

bus(i).ia2 $=0$;

bus(i).ia0 $=0$;

bus(i).i(1) $=0$;

bus(i).i(2) $=0$; 
- Calculate the voltages in each phase at each bus during the fault. $i=$ fault.bus_no;

for $j=1$ : length (bus)

\% Calculate the positive-, negative-, and zero-sequence voltages o in phase a at each bus during the fault. bus $(j) \cdot \operatorname{val}=$ bus $(j) \cdot \operatorname{volts}-$ bus $(i) \cdot$ ial * Zbusl $(j, i) ;$ bus $(j) \cdot \operatorname{va} 2=\quad-$ bus $(i) \cdot i a 2 *$ Zbus2 $(j, i) ;$ $\operatorname{bus}(j) \cdot \mathrm{vaO}=\quad-\operatorname{bus}(i) \cdot \mathrm{ia} 0 * \operatorname{zbus} 0(j, i) ;$

o Calculate the voltages in each phase at each bus during the fault \% (v(1) - voltage in phase $\mathrm{a} ; \mathrm{v}(2)$ - voltage in phase $\mathrm{b} ; \mathrm{v}(3)$ o voltage in phase c). $\operatorname{bus}(j) \cdot v(1)=\operatorname{bus}(j) \cdot \operatorname{val}+\operatorname{bus}(j) \cdot \operatorname{va} 2+\operatorname{bus}(j) \cdot v a 0 ;$ $\operatorname{bus}(j) \cdot v(2)=a 2 * \operatorname{bus}(j) \cdot v a 1+a * \operatorname{bus}(j) \cdot v a 2+\operatorname{bus}(j) \cdot v a 0 ;$ $\operatorname{bus}(j) \cdot v(3)=a * \operatorname{bus}(j) \cdot v a 1+a 2 * \operatorname{bus}(j) \cdot v a 2+\operatorname{bus}(j) \cdot v a 0 ;$ end 
\% Calculate the positive-, negative-, and zero-sequence voltages

\% in phase a at each bus during the fault.

bus $(j) \cdot \operatorname{val}=\operatorname{bus}(j) \cdot \operatorname{volts}-\operatorname{bus}(i) \cdot i a 1 *$ Zbusl $(j, i)$;

$\operatorname{bus}(j) \cdot \operatorname{va} 2=$

- $\operatorname{bus}(i) \cdot i a 2 *$ Zbus2 $(j, i)$;

$\operatorname{bus}(j) \cdot \operatorname{vaO}=$

- bus(i).ia0 * Zbus0 $(j, i)$;

\% Calculate the voltages in each phase at each bus during the fault

\% (v(1) - voltage in phase a; v(2) - voltage in phase b; v(3) -

ovoltage in phase $c)$.

$\operatorname{bus}(j) \cdot v(1)=\operatorname{bus}(j) \cdot \operatorname{val}+\operatorname{bus}(j) \cdot \operatorname{va}+\operatorname{bus}(j) \cdot v a 0 ;$

$\operatorname{bus}(j) \cdot v(2)=a 2 \star \operatorname{bus}(j) \cdot \operatorname{va} 1+a * \operatorname{bus}(j) \cdot v a 2+\operatorname{bus}(j) \cdot v a 0 ;$

bus $(j) \cdot v(3)=a *$ bus $(j) \cdot v a 1+a 2 *$ bus $(j) \cdot v a 2+$ bus $(j) \cdot v a 0 ;$

end

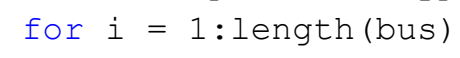




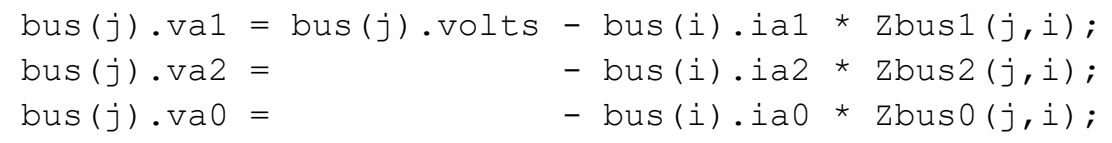


1223 function report(system, bus, line, Ybusl, fault)

1224 \% Function report displays the voltages and fault currents in each phase

1225 ot each bus and in each transmission line in the power system for

1226 o symmetrical three-phase faults in the subtransient, transient, or

1227 o steady-state periods.

1228

1229 \% Validate the number of input arguments in the call to the currently

1230 \% executing function.

1231 narginchk $(5,5)$;

1232

1233 obtain the number of buses in the power system.

1234 numBus $=$ length (bus);

1235

1236 Display the voltages and fault currents in each phase at each bus and in 1237 \% each transmission line in the power system for the particular type of

1238 \% fault being analyzed.

1239 fprintf('\n');

1240 fprintf('

1241 system.name);

1242 fprintf('os at Bus os $\backslash n$ ', fault.type_str, fault.bus_name);

1243 fprintf('Calculating $\%$ s Currents $\backslash n$ ', fault.calc_str);

1244 fprintf('|----------------Bus Information----------------' );

1245 fprintf('|-----Line Information----- |\n') ;

1246 fprintf(') Bus Volts / angle Amps / angle ');

1247 fprintf(') To | Amps / angle | \n');

1248 fprintf(') no. Name (pu) (deg) (pu) (deg) ');

1249 fprintf('। Bus | (pu) (deg) $\left.\mid \backslash \mathrm{n}^{\prime}\right)$;

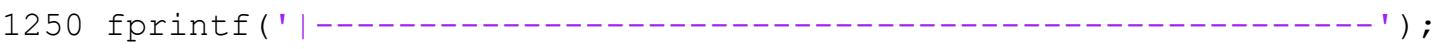

1251 fprintf('--

1252

1253 ․ Display the bus and line information for each bus and each transmission

1254 ine in the power system.

1255 for $i=1$ :numBus

1256

1257

1258

1259

1260

1261

1262

\% Display the bus number for each bus in the power system. fprintf(' $\% 3 d^{\prime}$, i) ; 


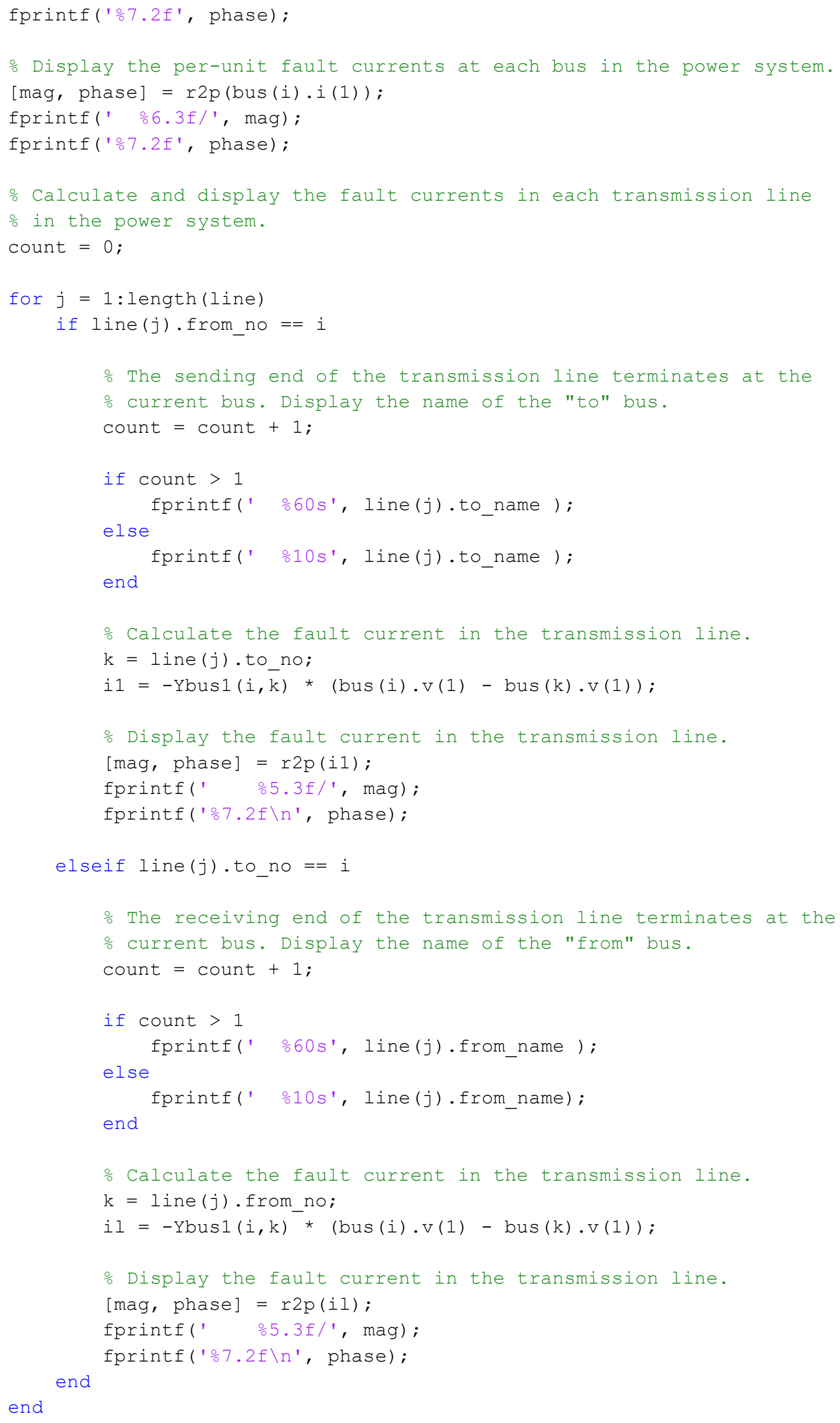


1321 end

1322

1323 fprintf('|--

1324 fprintf('--

1325

1326

1327

1328 function report_2(system, bus, line, Ybus1, Ybus2, Ybus0, fault)

$1329 \div$ Function report_2 displays the voltages and fault currents in each phase

1330 \% at each bus and in each transmission line in the power system for

1331 o unsymmetrical single line-to-ground, line-to-line, or double line-to-

1332 \% ground faults in the subtransient, transient, or steady-state periods.

1333

1334 V Validate the number of input arguments in the call to the currently

$1335 \%$ executing function.

1336 narginchk $(7,7)$;

1337

1338 \% Define the 'a' constant to represent the operation of shifting a phasor

$1339 \%$ by an angle of 120 degrees. This operation is equivalent to multiplying

1340 \% the phasor by the 'a' constant defined below and each multiplication by

1341 \% the 'a' constant rotates the phasor by an angle of 120 degrees without

1342 \% changing its magnitude.

$1343 \mathrm{a}=-0.5+1 j * 0.86602540378444 ;$ Rotate phasor by 120 degrees

1344 a2 $=-0.5-1 j * 0.86602540378444 ;$ : Rotate phasor by 240 degrees

1345

1346 을 Define the phases of a three-phase power system.

$1347 \mathrm{ph}(1)=$ 'a';

\% Phase a

1348 ph $(2)=$ 'b';

\% Phase b

$1349 \mathrm{ph}(3)=\mathrm{CC}^{\prime}$;

\% Phase C

1350

1351 obtain the number of buses in the power system.

1352 numBus = length (bus);

1353

1354 Display the voltages and fault currents in each phase at each bus and in

1355 \% each transmission line in the power system for the particular type of

1356 \% fault being analyzed.

1357 fprintf('\n');

1358 fprintf('

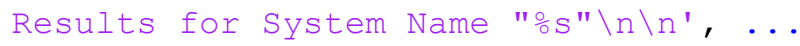

1359 system.name);

1360 fprintf('\%s at Bus os $\backslash n$ ', fault.type_str, fault.bus_name);

1361 fprintf('Calculating $\% s$ Currents $\backslash n$ ', fault.calc_str);

1362 fprintf('|----------------Bus Information-----------------');

1363 fprintf('|------Line Information------|\n');

1364 fprintf(') Bus P Volts / angle Amps / angle ');

1365 fprintf(') To | P Amps / angle | \n');

1366 fprintf(') no. Name h (pu) (deg) (pu) (deg) ');

1367 fprintf(') Bus | $\mathrm{h}$ (pu) (deg) $\left.\mid \backslash \mathrm{n}^{\prime}\right)$;

1368 fprintf('|----------------------------------------------' ) ;

1369 fprintf('------------------------ $\left.\backslash \mathrm{n}^{\prime}\right)$;

1370

1371 ․ Display the bus and line information for each bus and each transmission

1372 \% line in the power system.

1373 for $i=1$ :numBus

1374 for $k=1: 3$

1375 


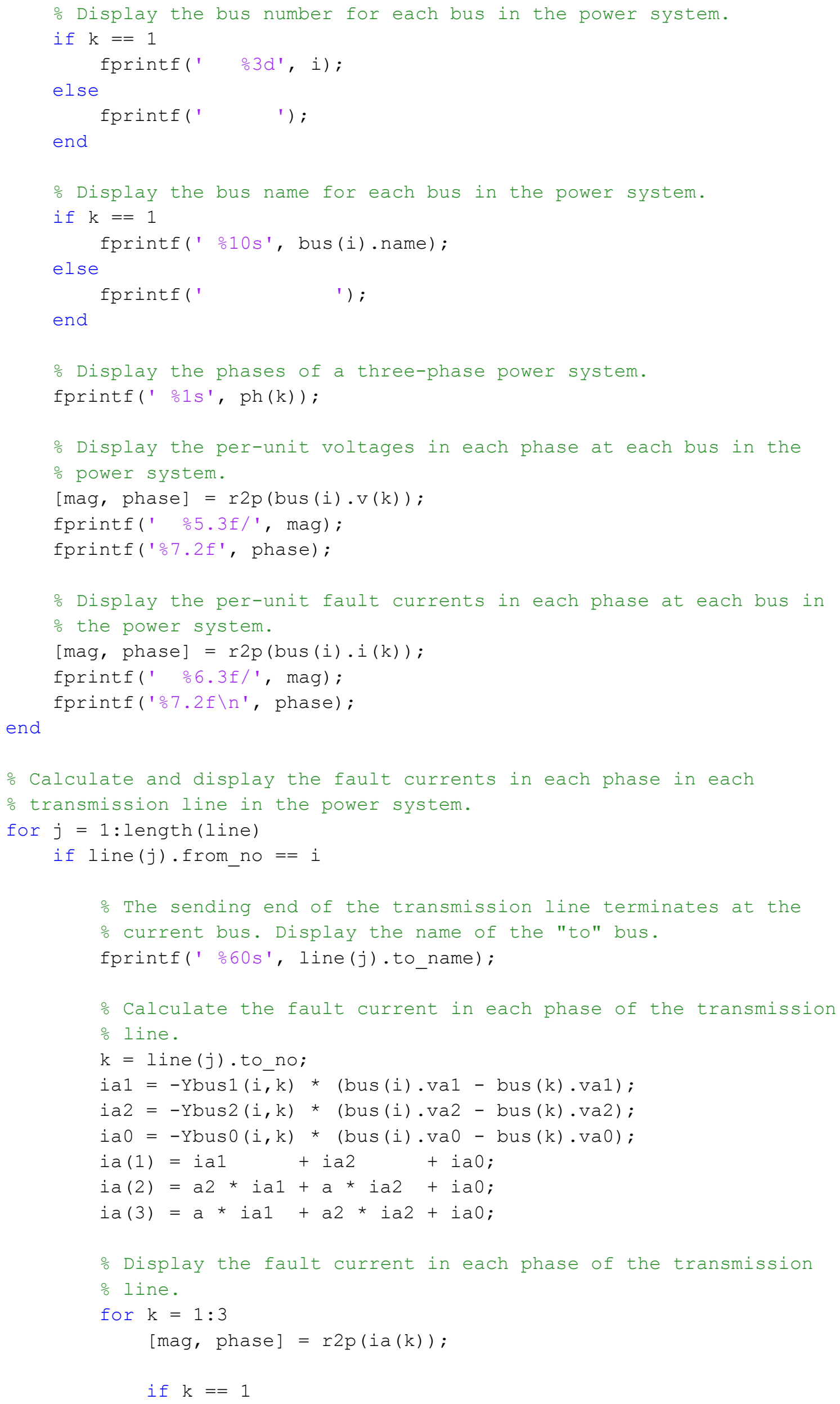


1431

1432

1433

1434

1435

1436

1437

1438

1439

1440

1441

1442

1443

1444

1445

1446

1447

1448

1449

1450

1451

1452

1453

1454

1455

1456

1457

1458

1459

1460

1461

1462

1463

1464

1465

1466

1467

1468

1469

1470 end

1471

1472 fprintf('

1473 fprintf('-

1474

1475

1476

1477 function [mag, phase] = r2p (rectangular)

1478 \% Function $2 \mathrm{p}$ converts a complex number in rectangular form into a complex $1479 \%$ number in polar form, with the phase angle specified in degrees.

1480

1481 Validate the number of input arguments in the call to the currently

1482 \% executing function.

1483 narginchk $(1,1)$;

1484

1485 \% Convert the complex number from rectangular form to polar form, with the 
1486 \% phase angle specified in degrees.

$1487 \mathrm{mag}=$ abs (rectangular); $\quad$ o Magnitude of complex number

1488 phase $=$ angle (rectangular) * $180 / \mathrm{pi} ; \quad$ o Phase angle (in degrees)

1489

1490 


\section{References}

[1] J. Grainger and W. Stevenson, Jr., Power System Analysis. New York, NY: McGraw-Hill, 1994.

[2] "IEEE Recommended Practice for Calculating Short-Circuit Currents in Industrial and Commercial Power Systems (IEEE Violet Book)," in IEEE Std 551-2006, 2006.

[3] S. Chapman, Electric Machinery and Power System Fundamentals. New York, NY: McGraw-Hill, 2002.

[4] "IEEE Recommended Practice for Industrial and Commercial Power Systems Analysis (IEEE Brown Book)," in IEEE Std 399-1997, 1997.

[5] N. Tleis, Power Systems Modelling and Fault Analysis: Theory and Practice. New York, NY: Elsevier, 2008.

[6] T. Short, Electric Power Distribution Handbook. Boca Raton, FL: CRC Press, 2014.

[7] Quanta Technology in association with Hydro One and Toronto Hydro, "Investigation of Solar PV Inverters Current Contributions during Faults on Distribution and Transmission Systems Interruption Capacity", 2012.

[8] M. Fischer and Â. Mendonça, "Representation of Variable Speed Full Conversion Wind Energy Converters for Steady State Short-Circuit Calculations," 2012 IEEE Power and Energy Society Transmission and Distribution Conference and Exposition, Orlando, FL, 2012, pp. 1-7.

[9] C. Opathella, B. N. Singh and B. Venkatesh, "A Review of Short-Circuit Models of Wind Generators," 2015 IEEE Electrical Power and Energy Conference (EPEC), London, ON, 2015, pp. 308-313.

[10] K. Saleh, H. Zeineldin and A. Al-Hinai, "A Three-Phase Fault Currents Calculation Method used for Protection Coordination Analysis," 2014 IEEE Power and Energy Society Transmission and Distribution Conference and Exposition, Chicago, IL, 2014, pp. 1-5.

[11] R. Walling, E. Gursoy and B. English, "Current Contributions from Type 3 and Type 4 Wind Turbine Generators During Faults," 2011 IEEE Power and Energy Society General Meeting, San Diego, CA, 2011, pp. 1-6. 
[12] ABB, "Faults in LVDC Microgrids with Front-End Converters", ABB Technical Application Papers, vol. 1, no. 14, pp. 1-65, 2015.

[13] J. Glover, M. Sarma and T. Overbye, Power System Analysis and Design, 5th ed. Stamford, CT: Cengage Learning, 2012.

[14] J. Das, Short-Circuits in AC and DC Systems: ANSI, IEEE, and IEC Standards. Boca Raton, FL: CRC Press, 2017.

[15] J. Das, Power System Analysis: Short-Circuit Load Flow and Harmonics. Boca Raton, FL: CRC Press, 2012.

[16] "IEEE Application Guide for AC High-Voltage Circuit Breakers Rated on a Symmetrical Current Basis," in IEEE Std C37.010-1979, 1979.

[17] C. Fortescue, "Method of Symmetrical Co-Ordinates Applied to the Solution of Polyphase Networks", Transactions of the American Institute of Electrical Engineers, vol. 37, no. 2, pp. 1027$1140,1918$.

[18] "IEEE Standard for Interconnection and Interoperability of Distributed Energy Resources with Associated Electric Power Systems Interfaces," in IEEE Std 1547-2018, 2018.

[19] J. Momoh, Smart Grid: Fundamentals of Design and Analysis. Hoboken, NJ: John Wiley \& Sons, 2012.

[20] General Electric Company, GE Industrial Power Systems Data Book. Schenectady, NY, 1978.

[21] "IEEE Standard for DC (3200 V and below) Power Circuit Breakers Used in Enclosures," in IEEE Std C37.14-2015, 2015.

[22] "Short-Circuit Currents in DC Auxiliary Installations in Power Plants and Substations," in IEC 61660-1, 1997.

[23] ETAP, "Validation Cases and Comparison Results", 2018. 


\section{Glossary}

AC symmetrical component of current: the ac symmetrical component (or steady-state component) of current is the sinusoidally varying component of fault current that starts out very high and decays to a steady-state value in two stages.

Asymmetrical current: The combination of the ac symmetrical component (or steady-state component) of current and the dc component of current.

Bolted fault: A fault with zero impedance; such faults produce the maximum possible fault currents and form the basis of calculations for withstand ratings on switching devices.

Circuit breaker: A switching device capable of making, carrying, and breaking currents under normal circuit conditions as well as making, carrying for a specified time, and breaking currents under specified abnormal conditions such as those that arise under fault conditions on a power system.

DC component of current: the dc component of current is the transient, nonperiodic, and exponentially decaying component of fault current that is created at the moment of the fault to satisfy the physical condition of continuous current at the instance of the fault since a current cannot change instantaneously in an inductive circuit; the level of the dc component of current depends on the instantaneous current in each phase at the instance of the fault.

Double line-to-ground fault: A fault that occurs when two phases of a power system come into direct contact with each other and with the ground or when flashover occurs between two phases of a power system and the ground.

Fault: Any failure that interferes with the normal flow of current to the loads. In most faults, a current path develops between two or more phases, or between one or more phases and the neutral (ground). Once this current path is established, it provides a short-circuit with relatively low impedance resulting in excessive current flows. Syn: short-circuit.

Fault analysis: The calculation of voltages and current flows under fault conditions on a power system.

Flashover: An ionized current path that occurs when the voltage difference between two or more phases, or between one or more phases and the neutral (ground), is large enough to ionize the surrounding air and produce an arc that establishes a current path between two or more phases, or between one or more phases and the neutral (ground). 
High-voltage: Circuit voltage over nominal $34.5 \mathrm{kV}$.

NOTE - ANSI standards are not unanimous in establishing the threshold of "high-voltage".

Impedance: The vector sum of resistance and reactance in an ac circuit.

Interrupting rating: the maximum current that an overcurrent protective device (e.g., circuit breaker) can interrupt or break at the voltage of the line in which it is placed.

Line-to-line fault: A fault that occurs when two phases of a power system come into direct contact or when flashover occurs between two phases of a power system.

Low-voltage: Circuit voltage under $1000 \mathrm{~V}$.

Medium-voltage: Circuit voltage greater than $1000 \mathrm{~V}$ up to and including $34.5 \mathrm{kV}$.

NOTE - ANSI standards are not unanimous in establishing the threshold of "high-voltage".

Method of symmetrical components: a technique introduced in 1918 by C.L. Fortescue for dealing with unbalanced polyphase systems called the method of symmetrical components. Fortescue's work proves that an unbalanced system of $n$ related phasors can be resolved into $n$ systems of balanced phasors called the symmetrical components of the original phasors. The $n$ phasors of each set of components are equal in magnitude and the phase displacement between adjacent phasors of the set are equal. According to Fortescue's theorem, three unbalanced phasors of a three-phase power system can be resolved into three balanced systems of phasors called the symmetrical components of the three-phase voltages or currents. See also: symmetrical components.

Negative-sequence: A set of symmetrical components consisting of three phasors equal in magnitude, displaced from each other by $120^{\circ}$ in phase, and having the opposite phase sequence from the original phasors. See also: symmetrical components.

Norton's theorem: states that a linear two-terminal circuit can be replaced by an equivalent circuit consisting of a current source in parallel with an impedance, where the current source is the short-circuit current through the terminals and the impedance is the input or equivalent impedance at the terminals when the independent sources are turned off. 
Permanent fault: A fault involving direct contact between two or more phases, or between one or more phases and the neutral (ground); will not clear even if power is removed from the faulted portion of the power system and then restored.

Positive-sequence: A set of symmetrical components consisting of three phasors equal in magnitude, displaced from each other by $120^{\circ}$ in phase, and having the same phase sequence as the original phasors. See also: symmetrical components.

rms: The square root of the average value of the square of the voltage or current over one period.

Sequence networks: The positive-sequence network is a per-phase equivalent circuit containing only the positive-sequence impedances and sources. The negative-sequence network is a per-phase equivalent circuit containing only the negative-sequence impedances, and the zero-sequence network is a per-phase equivalent circuit containing only the zero-sequence impedances.

Sequence impedances: The impedance of a circuit to positive-sequence current is called the positivesequence impedance of the circuit. Similarly, the impedance of the circuit to negative-sequence current is called the negative-sequence impedance of the circuit, and the impedance of the circuit to zero-sequence current is called the zero-sequence impedance of the circuit.

Short-circuit: An abnormal connection (including an arc) of relatively low impedance, whether made accidentally or intentionally, between two points of different potentials. Syn: fault.

Single line-to-ground fault: A fault that occurs when a single phase of a power system comes into direct with the ground or when flashover occurs between a single phase of a power system and the ground.

Source transformation: a source transformation is the process of replacing a voltage source in series with an impedance by a current source in parallel with an impedance or vice versa.

Subtransient current: The ac rms current flowing during the subtransient period is called the subtransient current. The subtransient currents are basically associated with the time constants of the amortisseur or damper windings of generators and motors.

Subtransient period: The ac symmetrical component of current starts out very high, and decays to a steady-state level in two stages. The first period of very rapid decay is called the subtransient period and it lasts only a few cycles. 
Subtransient reactance: The reactances of generators and motors in the subtransient period are called subtransient reactances. The resistances and reactances of transmission lines, transformers, and other static components do not change during the fault transients.

Superposition: The superposition principle states that the voltage across (or current through) an element in a linear circuit is the algebraic sum of the voltages across (or current though) that element due to each independent source acting alone.

Symmetrical three-phase fault: A fault that occurs when all three phases of a power system come into direct or when flashover occurs between all three phases of a power system, in which the magnitude and phase displacement between any two phases of voltages and currents are identical in each phase of the power system.

Symmetrical components: A symmetrical set of three vectors used to mathematically represent an unsymmetrical set of three-phase voltages or currents. In a three-phase system, one set of three equal magnitude vectors displaced from each other by $120^{\circ}$ in the same sequence as the original set of unsymmetrical vectors. This set of vectors is called the positive-sequence components. A second set of three equal magnitude vectors displaced from each other by $120^{\circ}$ in the reverse sequence as the original set of unsymmetrical vectors. This set of vectors is called the negative-sequence components. A third set of three equal magnitude vectors displaced from each other by $0^{\circ}$. This set of vectors is called the zerosequence components.

Synchronous reactance: The reactances of generators and motors in the steady-state period are the machines' synchronous reactances. The resistances and reactances of transmission lines, transformers, and other static components do not change during the fault transients.

Thevenin's theorem: states that a linear two-terminal circuit can be replaced by an equivalent circuit (called the Thevenin equivalent circuit) consisting of a voltage source in series with an impedance, where the voltage source is the open-circuit voltage at the terminals and the series impedance is the input or equivalent impedance at the terminals when the independent sources are set to zero.

Transient current: The ac rms current flowing during the transient period is called the transient current. The transient currents are basically associated with the time constants of the field windings of generators and motors. 
Transient fault: A fault involving flashover between two or more phases, or between one or more phases and the neutral (ground); usually clears if power is removed from the faulted portion of the power system for a short time and then restored.

Transient period: The ac symmetrical component of current starts out very high, and decays to a steadystate level in two stages. The second period of slower decay is called the transient period and it lasts for 0.5 to 1.0 second.

Transient reactance: The reactances of generators and motors in the transient period are called transient reactances. The resistances and reactances of transmission lines, transformers, and other static components do not change during the fault transients.

Unsymmetrical fault: A single line-to-ground, line-to-line, or double line-to-ground fault in which the magnitude and phase displacement between any two phases of voltages and currents in the three phases of the power system will differ.

Withstand rating: The maximum current that an unprotected electrical component can sustain for a specified period of time without the occurrence of extensive damage.

Zero-sequence: A set of zero-sequence components consisting of three phasors equal in magnitude and phase (i.e., zero phase displacement from each other). See also: symmetrical components. 